\title{
Cold Collisions of Atoms and Molecules
}

\author{
Marius Lysebo
}

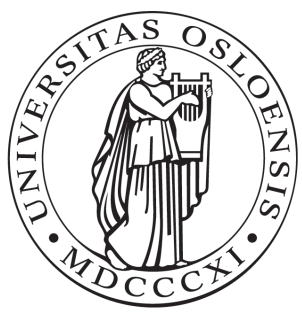

Thesis submitted for the degree of

Philosophiae Doctor

Department of Physics

University of Oslo

March 2010 
(C) Marius Lysebo, 2010

Series of dissertations submitted to the

Faculty of Mathematics and Natural Sciences, University of Oslo No. 957

ISSN 1501-7710

All rights reserved. No part of this publication may be

reproduced or transmitted, in any form or by any means, without permission.

Cover: Inger Sandved Anfinsen.

Printed in Norway: AiT e-dit AS.

Produced in co-operation with Unipub.

The thesis is produced by Unipub merely in connection with the thesis defence. Kindly direct all inquiries regarding the thesis to the copyright holder or the unit which grants the doctorate. 


\section{Acknowledgements}

I am deeply indebted to Leif Veseth for excellent supervision during the last few years. I thank you for the relevant and critical feedback you always provided and for the many discussions we have had. In particular I want to thank you for your patience during the long period when I was struggling with the numerical implementation of matrix elements, basis transformations, symmetries, etc. Not once did you suggest that the pace was slow, although the visible output was non-existent.

I am grateful to my co-supervisor Morten Hjorth-Jensen. Unfortunately time did not permit us to do research together. However, I thank you for letting me be a part of your excellent Computational Physics course. The numerous discussions about projects on a variety of subjects, from tsunami waves via degenerate stars to the time evolution of the Schrödinger equation, broadened my knowledge of physics and computational methods in general, and certainly contributed to this thesis. I also thank you for all the coffee, the whiskey, and the laughs in your office.

I would also like to thank Susanne F. Viefers for educational and inspiring collaboration on FYS2140 during the last few years. Your democratic leader style was highly motivating and deeply appreciated.

I also want to thank a few of my fellow students and friends: Ken Tore Tallakstad for all the long lunches, the coffee-breaks, the occasional gossip and for the exchange of dubious internet links. Per Øyvind Sollid for sharing with me a particular interest for the less important things in life. Our numerous discussions on a broad range of topics were treasured, as was your careful proofreading of this manuscript. Theresa Palmer was a never ending source of good spirit and provided valuable help with the subtleties of the English language

Finally, thank you Steinar Sagen, Tore Sagen and Bjarte Tjøstheim for the countless number of hours I have spent listening to you while coding. Even debugging became truly enjoyable in your company.

...we have the easy legged humor, you know...

Steinar Sagen, June $9^{\text {th }} 2009$ 



\section{Preface}

The first five chapters of this thesis are meant to serve as an introduction to the world of cold atoms and diatomic molecules. This is a vast area of research, and a rather "hot topic" in modern atomic, molecular and optical physics. The presentation provided here is of course strongly biased by my own view of the field, and how I perceive that it ought to be introduced. In addition, the introduction should serve as a primer to the papers that represent the actual research undertaken by us during the last four years (August 2006 - March 2010).

The introductory part is written at a level suitable for physics students working in other areas of physics. The first chapter should be readable for everyone with a basic knowledge of physics, while the later chapters could benefit from advance knowledge of atomic and molecular physics.

Chapter 1 introduces cold atoms, while chapter 2 introduces cold diatomic molecules and their quantum mechanical description. Cold collisions are treated in chapter 3.

Chapters 4 and 5 introduce the work presented in the papers, and are meant to provide background information and motivations. However, we do not repeat the arguments or the conclusions presented in these papers, we merely introduce the approach to the problem. Papers I and IV are related to chapter 4, whereas papers II and III are related to the material presented in chapter 5.

\section{Motivations for the present work}

The motivations for going into the world of cold atoms and molecules are many. Some of the questions whose answers are sought are:

- How can cold atoms and molecules be created?

- How do cold atoms and molecules collide?

- How can we control cold collisions by external fields? 
- Can we perform high-resolution molecular spectroscopy?

- Can we expect new states of matter at very low temperatures?

- Can chemical reactions at extremely low temperature be controlled?

- Does the electron have an electric dipole moment?

- Can we make a quantum computer out of cold atoms or molecules?

Partial answers to a few of these questions have been provided, some even in this thesis, whereas the majority of these questions are subjects of active and current research by many groups worldwide.

The present work has relied heavily on numerical methods. Quite extensive programs have been developed from scratch in $\mathrm{C} / \mathrm{C}++$. However, the numerical methods and the algorithms have not been described in this thesis. The majority of the algorithms we have used are well tested and can be found in standard references. Some parts of the code are however not standard. This applies in particular to the implementation of various matrix elements, different molecular basis sets and the transformations between them, the incorporation of the Pauli principle and so on. Too many weekends have elapsed testing and debugging these codes, if time permits they may become available for everyone to modify or use in the future. At the present, the user interface is not friendly enough. As these codes merely combine known results, a more detailed description was not included in the papers, whereas such a description seems far to technical to be appropriate for the first part of this thesis. 


\section{List of papers}

Paper I: Marius Lysebo and Leif Veseth,

Cold collisions between atoms and diatomic molecules,

Phys. Rev. A 77, 032721 (2008)

Paper II: Marius Lysebo and Leif Veseth,

Ab initio calculation of Feshbach resonances in cold atomic collisions:

$s$ - and $p$-wave Feshbach resonances in ${ }^{6} \mathrm{Li}_{2}$,

Phys. Rev. A 79, 062704 (2009)

Paper III: Marius Lysebo and Leif Veseth,

Feshbach resonances and transition rates for cold homonuclear collisions between ${ }^{39} \mathrm{~K}$ and ${ }^{41} \mathrm{~K}$ atoms,

Phys. Rev. A. 81, 032702 (2010)

Paper IV: Håkon Bjørgen, Marius Lysebo and Leif Veseth,

Index of refraction for cold lithium- and diatomic sodium matter waves traveling through cold noble gases,

Submitted to Phys. Rev. A 



\section{Contents}

I Introduction 1

1 Cold atoms 3

1.1 Cooling atoms in one dimension . . . . . . . . . . . . . . . . . . 4

1.2 Cold atomic gases . . . . . . . . . . . . . . . . . . . 5

1.2.1 Optical molasses . . . . . . . . . . . . . . . 5

1.2.2 The magneto-optical trap (MOT) . . . . . . . . . 7

1.2.3 Evaporative cooling . . . . . . . . . . . . . . . . . . 9

1.3 Further reading . . . . . . . . . . . . . . . . . . . . 12

2 Diatomic Molecules $\quad 13$

2.1 The Born-Oppenheimer approximation . . . . . . . . . . . 13

2.2 Born-Oppenheimer potential curves . . . . . . . . . . . 16

2.2.1 Comments on the Møller-Plesset perturbation theory . 17

2.2 .2 Basis sets . . . . . . . . . . . . . . 18

2.2.3 Potential curves at long range . . . . . . . . . . . . . 19

2.3 Vibration and rotation . . . . . . . . . . . . . . . . . . 19

2.4 Cold and ultracold molecules . . . . . . . . . . . . . . 21

2.4.1 Obtaining cold molecules . . . . . . . . . . . . 23

2.4.2 Photoassociation (PA) of diatomic molecules . . . . . 24

2.4.3 Play pool to stay cool . . . . . . . . . . . . . . 25

2.5 Further reading . . . . . . . . . . . . . . . . . 26

3 Cold collisions $\quad 29$

3.1 Elastic scattering . . . . . . . . . . . . . . . . . 30

3.1.1 The phase shift . . . . . . . . . . . . . . . 35

3.1 .2 The low energy limit . . . . . . . . . . . . . . . . 36

3.1.3 Levinson's theorem . . . . . . . . . . . . . . . . . . . . . . 38

3.1.4 The optical theorem . . . . . . . . . . . . . . . 40

3.2 Inelastic scattering . . . . . . . . . . . . . . . . . . 40

3.2.1 The multichannel equations . . . . . . . . . . . . . 41

3.2.2 The $S$-matrix . . . . . . . . . . . . . . . . . . . . . 43 
3.3 Further reading . . . . . . . . . . . . . . . . . . . . . . 44

4 Collisions between diatomic molecules and atoms 45

4.1 Description of the buffer-gas cooling process . . . . . . . . 46

4.2 Buffer-gas cooling and the present work . . . . . . . . . . . . 48

4.2.1 Cooling of the rotational degree of freedom . . . . . . . 51

4.3 Matter wave interferometry and the index of refraction . . . . 52

4.4 Further reading . . . . . . . . . . . . . . . . . 55

5 Atomic collisions and Feshbach resonances 57

5.1 Toy model of a Feshbach resonance . . . . . . . . . . . . . 57

5.1 .1 Long range solution . . . . . . . . . . . . . . . 58

5.1 .2 Short range solution . . . . . . . . . . . . . . . . 59

5.1.3 A Feshbach resonance appears . . . . . . . . . . . 61

5.2 Feshbach resonances in diatomic alkali molecules . . . . . . . . 63

5.2 .1 Our work .................. . 64

5.3 Applications of Feshbach resonances . . . . . . . . . . . . . 65

5.3 .1 Molecular BEC . . . . . . . . . . . . . . . 66

5.3 .2 BCS-BEC crossover . . . . . . . . . . . . 67

5.4 Further reading . . . . . . . . . . . . . . . . . . 68

$\begin{array}{ll}\text { Bibliography } & 79\end{array}$

$\begin{array}{lr}\text { II Papers } & 81\end{array}$ 


\section{Part I}

\section{Introduction}





\section{Chapter 1}

\section{Cold atoms}

The 1997 Noble prize in physics was awarded Steven Chu, Claude CohenTannoudji and William D. Phillips for developing methods to cool and trap atoms with laser light. Without their achievements, the present work would merely be of theoretical interest, without experimental relevance. In chapter 1 we review common cooling methods used for atoms today. In chapter 2 we introduce the diatomic molecule and methods for cooling it.

The present work is concerned with cold and ultracold gases of atoms and diatomic molecules. We shall reserve the word cold for temperatures below one kelvin, and the word ultracold for temperatures below one microkelvin. The atoms and molecules that we consider are neutral.

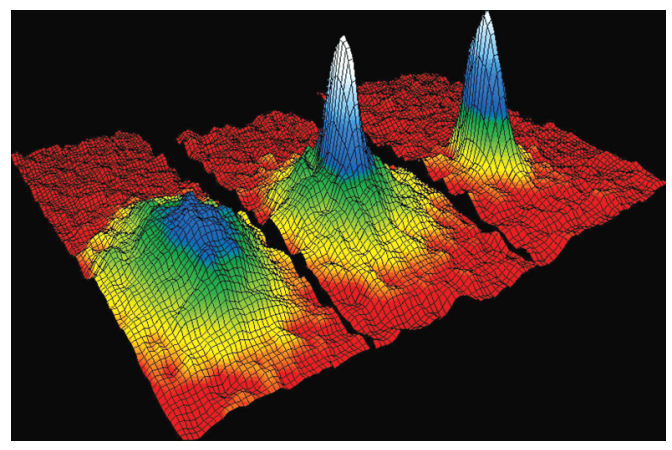

Figure 1.1: Bose-Einstein condensate of rubidium atoms cooled to 170 nanokelvin. The figure shows the velocity distribution at three different temperatures.

One important question to answer is this: What does it mean to cool 
a gas of atoms? Cooling means removal of energy, both the kinetic energy and the internal energy. The temperature is related to the width of the velocity distribution in a three dimensional atomic gas. Therefore the velocity distribution of a group of cold atoms should be narrow, that is, the velocities of the atoms should be similar and as small as possible (see Fig. 1.1).

\subsection{Cooling atoms in one dimension}

Neutral atoms are cooled to low temperatures using lasers. The basic principle is very easy to understand as it relies on momentum conservation. The ideal atoms for laser cooling have two internal states $|1\rangle$ and $|2\rangle$, with an energy separation $\hbar \omega_{0}$. A photon with frequency $\omega \simeq \omega_{0}$ can then excite an atom from state $|1\rangle$ to state $|2\rangle$, see Fig. 1.2.

If we let the photon propagate in the opposite direction of the atom, momentum conservation gives

$$
m v-\frac{\hbar \omega}{c}=m v^{\prime}
$$

where $v$ is the speed of the atom before the collision and $v^{\prime}$ is the speed of
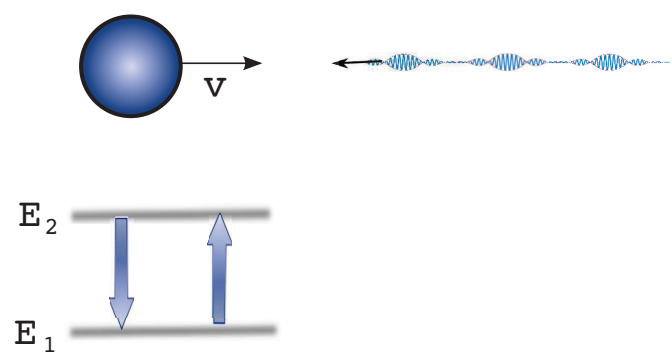

Figure 1.2: An atom with velocity $v$ absorbs a photon propagating in the opposite direction. The atom is excited to state $|2\rangle$ with energy $E_{2}$ during the absorption process. A short time later the atom returns to state $|1\rangle$ by spontaneous emission.

the atom after the collision. Hence, the change in velocity is

$$
\Delta v=-\frac{\hbar \omega}{m c},
$$

which is of the order $10^{-2} \mathrm{~m} / \mathrm{s}$. 
The atoms return to the ground state via spontaneous emission. The photon emitted in this process has no preferred direction, therefore, after a large number of absorption-emission processes, they do not give a net acceleration. The lifetime of the excited state $|2\rangle$ may be 10-20 nanoseconds, which gives an deceleration $a=-10^{6} \mathrm{~m} / \mathrm{s}^{2}$. At room temperature the most probable velocity of an atom is $\simeq 1000 \mathrm{~m} / \mathrm{s}$, hence the stopping distance with the aforementioned acceleration is around $1 \mathrm{~m}$. The lifetime of the excited state is of course a property of the individual atom that varies with the different species. However, for laser cooling to be effective, the lifetime of the excited state $|2\rangle$ must be short.

\subsection{Cold atomic gases}

\subsubsection{Optical molasses}

Three dimensional atomic gases may be cooled using six orthogonal laser beams, see Fig. 1.3. In many respects this is a rather natural generalization

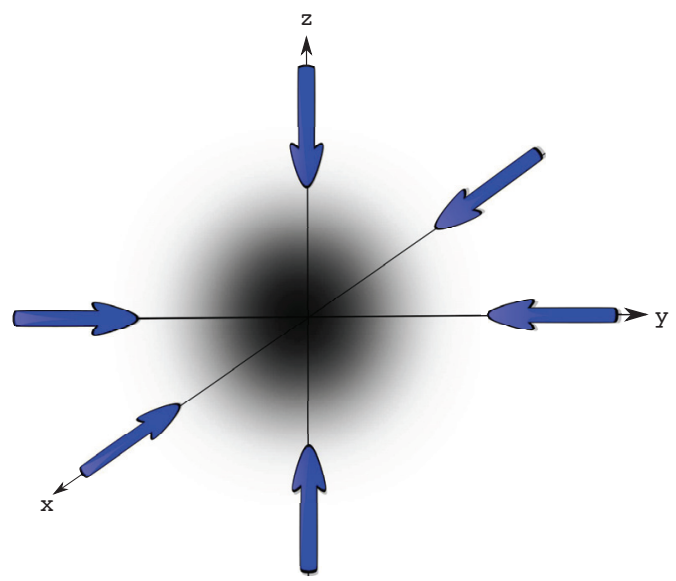

Figure 1.3: Simplified sketch of the optical molasses technique. The blue arrows are lasers emitting photons with an energy $\hbar \omega$. The lasers emit photons with the same frequency lower than the resonance frequency $\omega_{0}=\frac{E_{2}-E_{1}}{\hbar}$.

of the setup used in one dimension. However, a few additional effects need to be considered and we start with the Doppler shift. 
Consider a photon with angular frequency $\omega$ measured in the laboratory system. In the rest frame of the atom, the angular frequency $\omega^{\prime}$ of the same photon is

$$
\omega^{\prime}=\omega-\mathbf{k} \cdot \mathbf{v},
$$

where $\mathbf{k}$ is the wave vector for the light and $\mathbf{v}$ is the velocity of the atom in the laboratory system. If $\mathbf{k}$ and $\mathbf{v}$ are anti-parallel, then $\omega^{\prime}>\omega$ and if $\mathbf{k}$ and $\mathbf{v}$ are parallel then $\omega^{\prime}<\omega$. This is the Doppler shift.

The Doppler shift is in fact utilized to cool atomic gases through the setup shown in Fig. 1.3. The frequency of the photons emitted from the lasers is adjusted so that it is smaller than the transition frequency between the internal states of the atoms, i.e. $\omega<\omega_{0}$. Therefore the atoms will absorb photons propagating in the opposite direction of $\mathbf{v}$ with a high probability, as these photons have a frequency that is Doppler shifted to match the resonance frequency. Likewise, the frequency of photons propagating parallel to $\mathbf{v}$ is Doppler shifted further out of resonance, and the probability of absorbing these photons will be low. This mechanism is schematically shown in Fig. 1.4 and is named the optical molasses technique. The force acting on atoms

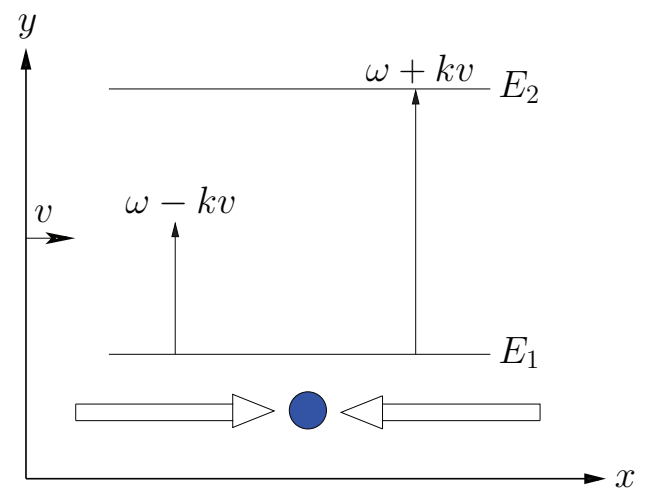

Figure 1.4: The principle behind the optical molasses technique. The two counter propagating laser beams shown as arrows. In the rest frame of the atom the laser light is Doppler shifted. The frequency of the photons propagating in the opposite direction of the atom is shifted closer to the resonance frequency $\omega_{0}$.

in such a setup can be written [1]

$$
\mathbf{F}=-\alpha \mathbf{v}
$$


where $\alpha$ is a positive constant that depends on the intensity of the light, the lifetime of the excited state and the resonance frequency $\omega_{0}$.

An atom can be cooled to absolute zero if we only consider the force in Eq. (1.4). However, this is a consequence of the simplified model we have used and is not observed in experiments. Spontaneous emission does not contribute with a net acceleration, but the recoil from photons emitted make the atom behave like a random walker in velocity space. The mean square displacement of the random walker equals the number of steps multiplied with the square of the step length. Hence, spontaneous emission causes the mean square velocity to increase. From these considerations it can be argued that there exists a lower attainable temperature $T_{D}$ for laser cooling of atoms. Detailed calculations [2] show that this limit, in the following referred to as the Doppler cooling limit, is

$$
k_{B} T_{D}=\frac{\hbar}{2 \gamma}
$$

where $k_{B}$ is Boltzmann's constant and $\gamma$ is the natural lifetime of the excited state $|2\rangle$.

For ten years, the lowest temperatures achievable with laser cooling were believed to be given by Eq. (1.5). However, in 1988 Lett et al. observed atoms laser cooled below the Doppler limit [3]. Lett et al. themselves wrote:

We have measured the temperature of a gas of sodium atoms released from "optical molasses" to be as low as $43 \pm 20 \mu \mathrm{K}$. Surprisingly, this strongly violates the generally accepted theory of Doppler cooling which predicts a limit of $240 \mu \mathrm{K}$.

This was soon resolved by J. Dalibard and C. Cohen-Tannoudji [4] and explained with a mechanism dubbed the Sisyphus effect. It is the light shift combined with polarization gradients that is involved in the Sisyphus effect. The detailed explanation is however somewhat subtle and will be omitted.

\subsubsection{The magneto-optical trap (MOT)}

The magneto-optical trap (MOT) is the workhorse in cold atomic physics. Compared to the optical molasses technique, the MOT has several advantages. Although atoms may be cooled to low temperates with the optical molasses technique, there is no restoring force acting on the atoms. In the MOT an inhomogeneous magnetic field provides trapping capabilities.

It is necessary to consider the internal states of an atom in a magnetic field to understand the working principle of a MOT. The inhomogeneous 


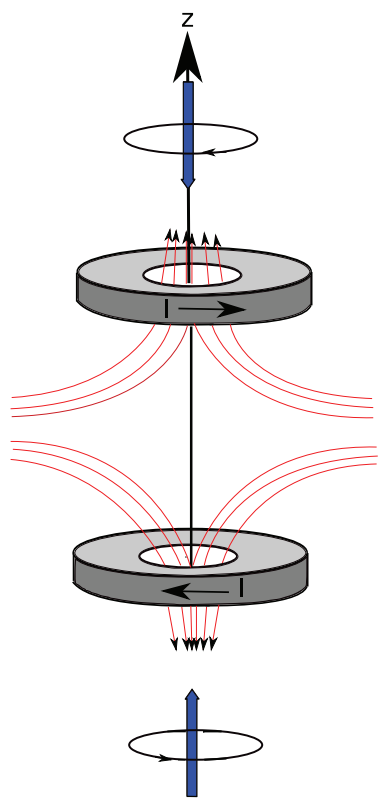

Figure 1.5: Sketch of the experimental setup known as a MOT. Two coils with currents $I$ create an inhomogeneous magnetic field. Magnetic field lines are indicated with red arrows. Three pairs of counter-propagating laser beams with different polarizations are used (only one pair of lasers indicated with blue arrows is shown).

magnetic field B is created by two coils with currents $I$ running in opposite directions, see Fig. 1.5. The magnetic field interacts with the atoms via the Zeeman interaction

$$
H_{\mathrm{Z}}=\mu_{B}\left(g_{s} \mathbf{S}+\mathbf{L}\right) \cdot \mathbf{B}=\mu_{B} \mathbf{M} \cdot \mathbf{B},
$$

where $\mathbf{S}$ is the total electronic spin, $\mathbf{L}$ is the electronic angular momentum and $\mathbf{M}$ is the magnetic moment of the system, omitting the small nuclear spin contribution. The Bohr magneton $\mu_{B}$ is equal to $5.788 \times 10^{-9} \mathrm{eV} / \mathrm{G}$ and the $g$-factor for the electron is $g_{s} / 2=1.00116$.

The first order Zeeman effect can be obtained from the Wigner-Eckart theorem [5]

$$
E_{\mathrm{Z}}=\left\langle\psi_{M_{F}}^{F}\left|H_{\mathrm{Z}}\right| \psi_{M_{F}}^{F}\right\rangle=\mu_{B} B \frac{\langle F|| M|| F\rangle}{\langle F|| F|| F\rangle} \hbar M_{F}=\hbar \mu_{B} B g_{F} M_{F},
$$


where the $g_{F}$-factor can be obtained by working out the reduced matrix element $\frac{\langle F|| M|| F\rangle}{\langle F|| F|| F\rangle}$. The atomic state with total angular momentum $F$ and projection $M_{F}$ on a space-fixed axis is denoted $\left|\psi_{M_{F}}^{F}\right\rangle$. The point is to notice the simple dependence on the $M_{F}$ quantum number in Eq. (1.7).

The magnetic field is zero in the middle of the trap (at $x=y=z=0$ ) and increases as [6]

$$
B(z)=A \sqrt{\rho^{2}+4 z^{2}},
$$

where $\rho^{2}=x^{2}+y^{2}$ and $A$ is a constant. The magnetic field changes direction at $z=0$ where it is zero.

Consider now atoms displaced from the center of the trap along the $z$ axis, where the magnetic field strength and the Zeeman energy $E_{\mathrm{Z}}$ increases linearly with $z$. For simplicity we consider an alkali atom with total electronic $\operatorname{spin} \mathbf{S}=\frac{1}{2}$, zero angular momentum $\mathbf{L}$ and nuclear $\operatorname{spin} \mathbf{I}=\frac{1}{2}$. There are then two possible quantized values for the total angular momentum quantum number $F$, i.e. $F=0 \vee F=1$.

Atoms in the $\left|F=1, M_{F}=-1\right\rangle$ state experience a negative Zeeman shift at $z>0$, whereas atoms in the $\left|F=1, M_{F}=+1\right\rangle$ state experience a positive Zeeman shift at $z>0$. Similar considerations hold for atoms displaced along the negative $z$-axis (but with reversed conclusions). The laser frequency $\omega$ is adjusted so that it is less than the resonance frequency $\omega_{0}$ (as in the optical molasses technique). Hence, atoms displaced from the origin along the positive $z$-axis have a higher probability of being excited to the $\left|F=1, M_{F}=-1\right\rangle$ state, compared to the states with $M_{F}=0$ or $M_{F}=1$, cf. Fig. 1.6. To facilitate absorption of photons propagating in the direction opposite of the atom, different polarizations are used on the two counter-propagating laser beams. The laser at the positive $z$-axis emits light with $\sigma^{-}$polarization, which only induces $\Delta M_{F}=-1$ transitions. The laser at the negative $z$-axis emits light with $\sigma^{+}$polarization which only induces $\Delta M_{F}=+1$ transitions. Hence, the atom is effectively pushed back towards $z=0$ when displaced from the origin. See Fig. 1.6 for an illustration of the principle behind a MOT.

\subsubsection{Evaporative cooling}

The different methods considered so far may be able to cool an atomic gas to temperatures around one microkelvin, i.e. to the so called recoil limit determined by the recoil energy. An atom absorbing or emitting a photon will experience a velocity recoil, and the energy associated with it is the recoil energy. Temperatures corresponding to the recoil energy are not sufficient 


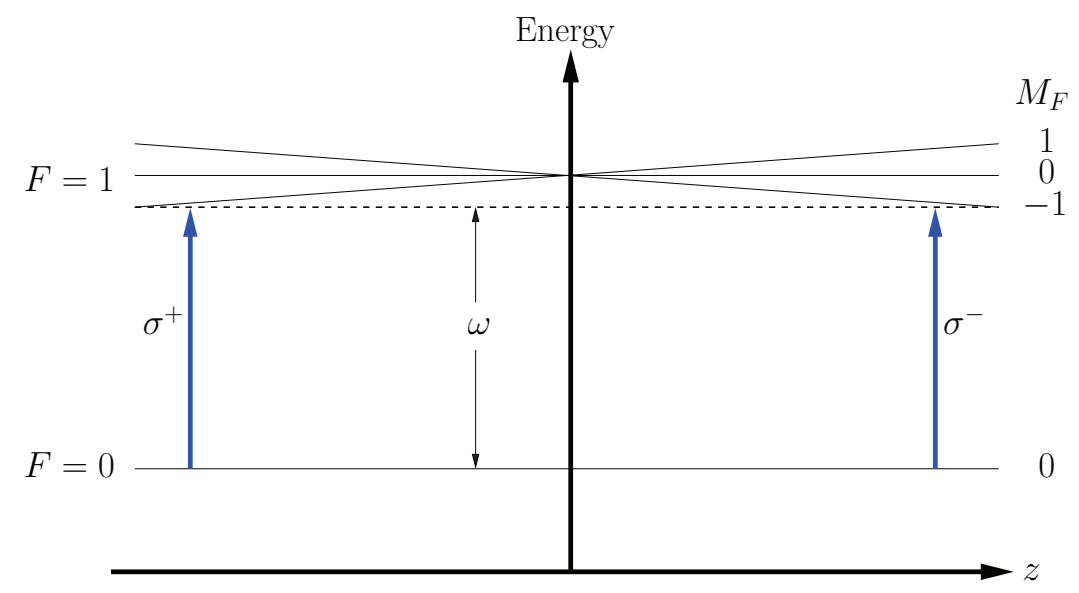

Figure 1.6: Hyperfine structure for an alkali atom in an inhomogeneous magnetic field. The magnetic field strength increases with $z$ and the magnetic field changes direction at $z=0$. The two counter-propagating laser beams along the $z$-axis have different polarization, specified as $\sigma^{-}$and $\sigma^{+}$polarization. The selection rules for $\sigma^{ \pm}$polarized light are $\Delta M_{F}= \pm 1$. Laser light is indicated with blue arrows in the present figure. See the text for a further discussion.

for Bose-Einstein condensation (BEC). To create a BEC, temperatures in the nanokelvin temperature range are required. Such low temperatures may be achieved using evaporative cooling, as demonstrated experimentally in June 1995 when the first Bose-Einstein condensate was observed [7].

With evaporative cooling we mean selective removal of the hottest atoms. We assume that the atoms are trapped in e.g. a MOT. The most energetic atoms can be removed from the system by lowering the potential, allowing the hottest atoms to escape, see Fig. 4.1. The remaining atoms are then allowed to thermalize, thereafter the cycle of removal and thermalization is repeated.

The thermalization process relies on elastic collisions between the atoms within the trap. Collisions between atoms where only kinetic energy is exchanged are called elastic collisions. The cross section for elastic collisions at low temperatures is given by [8]

$$
\sigma_{\text {elastic }}=4 \pi|\alpha|^{2},
$$

where $\alpha$ is a complex number that depends on the atomic species. The alkali 

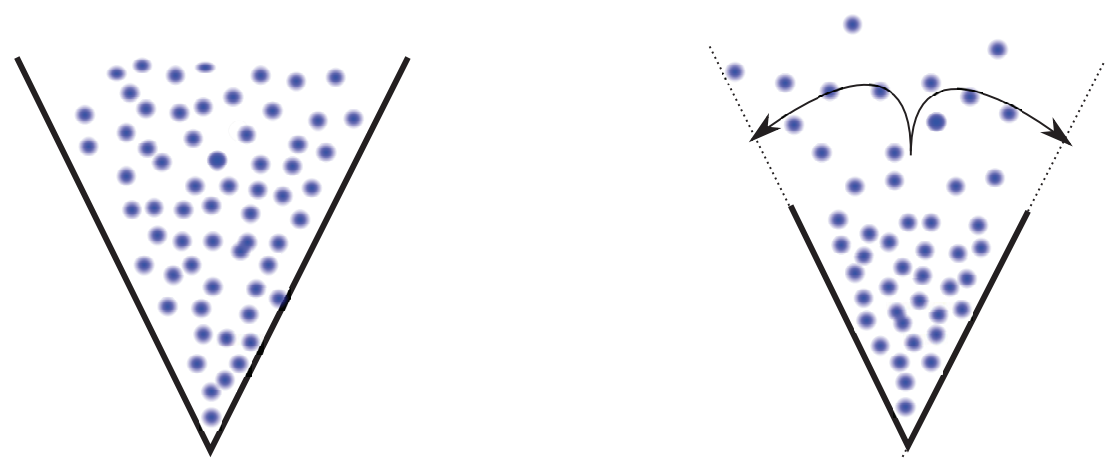

Figure 1.7: The principle behind evaporative cooling. Atoms (blue spheres) are trapped by a linear potential. When the the strength of the potential is decreased, the most energetic atoms are allowed to escape.

atoms have a large scattering length and therefore a large elastic cross section. Surprisingly, the elastic cross section is four times what one expects from a naive geometrical consideration, interpreting the scattering length $\alpha$ as the radius of a sphere. We also remark that for identical bosons the elastic cross section is twice the value given in Eq. (1.9). We will return to the scattering length in chapter 3 .

Collisions where part of the internal energy is exchanged with kinetic energy are called inelastic collisions. Inelastic collisions are not favorable for evaporative cooling. Clearly, if the internal energy is exchanged for kinetic energy the atoms may heat up. It is not uncommon that the trapping potential felt by an atom depends on the internal state of the atom. During a collision, if the internal state is changed to an untrappable state, the atom is lost from the trap. The cross section for the unfavorable inelastic collisions is $[8]$

$$
\sigma_{\text {inelastic }}=\frac{4 \pi|\alpha|}{k},
$$

where $\alpha^{\prime}$ is a real number determined from the scattering amplitude (see chapter 3) and $k$ is the wave number

$$
k=\frac{\sqrt{2 m E}}{\hbar} .
$$

The point here is that the cross section for inelastic collisions is inversely proportional to $k$. As the energy and $k$ decreases, the inelastic cross section 
increases. For the evaporative cooling process to be effective, the ratio of elastic to inelastic collisions needs to be high. The fact that $\sigma_{\text {inelastic }}$ increases when the temperature decreases limits the final temperature achievable with evaporative cooling.

It was commented that evaporative cooling is often used to produce BoseEinstein condensates. For such experiments the phase space density is equally as important as the temperature. If the volume occupied by the remaining atoms is reduced to an extent that overcompensates for the atoms lost during the evaporative cooling process, the phase space density will increase. Eventually, this together with the low temperature may lead to the creation of BECs.

One of the coldest atomic gases ever observed was created at MIT in 2003, and reported in the September edition of Science the same year [9]. The temperature was measured to be $450 \pm 80$ picokelvin.

\subsection{Further reading}

In preparing this chapter, the book "Laser Cooling and Trapping" by Metcalf and van der Straten [10], along with "Atomic Physics" by Foot [1], were used. Together with the extensive list of references included, these two books review the topics relevant to the field of laser cooling of atoms. References to more advanced treatments are also included, in particular in the book by Metcalf and van der Straten. 


\section{Chapter 2}

\section{Diatomic Molecules}

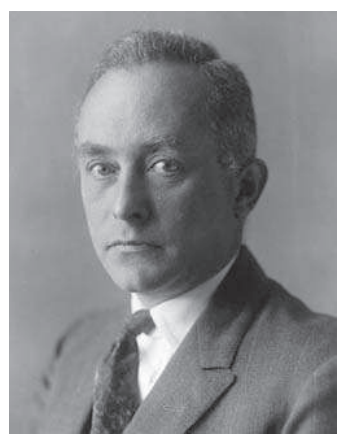

Figure 2.1: Max Born (11 December 1882 - 5 January 1970) won the Nobel Prize in Physics in 1954 for his statistical interpretation of the wave function. Together with J. Robert Oppenheimer he authored a paper introducing the Born-Oppenheimer approximation in 1927.

Throughout this thesis we will work within the Born-Oppenheimer approximation, let us therefore start this chapter by introducing it. We work in atomic units where $\hbar=m_{e}=e=1$. The electron mass is denoted by $m_{e}$ and the electron charge unit is denoted by $e$.

\subsection{The Born-Oppenheimer approximation}

The Born-Oppenheimer approximation describes the separation of the electronic motion from the nuclear motion. The approximation is named after 
Max Born and J. Robert Oppenheimer, and was introduced in a famous article published in Annalen der Physik in 1927 [11].

The natural starting point is the Hamiltonian, and for a diatomic molecule it can be written

$$
H=-\frac{1}{2 m_{A}} \nabla^{2}\left(\mathbf{r}_{A}\right)-\frac{1}{2 m_{B}} \nabla^{2}\left(\mathbf{r}_{B}\right)-\frac{1}{2} \sum_{i=1}^{N} \nabla^{2}\left(\mathbf{r}_{i}\right)+V\left(\mathbf{r}_{A}, \mathbf{r}_{B}, \mathbf{x}\right)+H_{\mathrm{rel}}\left(\mathbf{r}_{A}, \mathbf{r}_{B}, \mathbf{x}\right),
$$

where $\mathbf{x}=\left[\mathbf{r}_{1}, \mathbf{r}_{2}, \mathbf{r}_{3}, \ldots, \mathbf{r}_{N}\right]$ and $N$ is the number of electrons. The first three terms of Eq. (2.1) is the kinetic energies for nuclei $A$ and $B$ and for the electrons respectively. The mass of nuclei $A$ is $m_{A}$ and the mass of nuclei $B$ is $m_{B}$. The vectors $\mathbf{r}_{A}$ and $\mathbf{r}_{B}$ point to the positions of nuclei $A$ and $B$ respectively, similarly, the vectors $\mathbf{r}_{i}$ points to electron $i$. The potential $V$ contains the potential energy from the electrostatic interactions and $H_{\text {rel }}$ contains the relativistic part which we will return to later.

Although the operator in Eq. (2.1) is easy to write down, the corresponding time-independent Schrödinger equation

$$
H \Psi\left(\mathbf{r}_{A}, \mathbf{r}_{B}, \mathbf{x}\right)=E \Psi\left(\mathbf{r}_{A}, \mathbf{r}_{B}, \mathbf{x}\right)
$$

is not solvable. Two transformations will be applied to the Hamiltonian in Eq. (2.1). The first transformation consists of separating out the center of mass motion. This separation is not exact when $H_{\text {rel }} \neq 0$, but the additional terms are of the order $v^{2} / c^{2}$ and are omitted. The second transformation allows us to consider the movement of the nuclei as a one-particle problem, described by the internuclear separation $r$ and the angles $\theta$ and $\phi$ that specifies the orientation of the internuclear axis.

After applying these transformations the Hamiltonian can be written

$$
\begin{aligned}
H & =-\frac{1}{2} \sum_{i=1}^{N} \nabla^{2}\left(\mathbf{r}_{i}\right)-\frac{1}{2\left(m_{A}+m_{B}\right)} \sum_{i, j=1}^{n} \nabla_{i} \cdot \nabla_{j}-\frac{1}{2 \mu} \nabla^{2}(r, \theta, \phi)+V(r, \mathbf{x}), \\
& =H_{\mathrm{e}}+H_{\mathrm{n}},
\end{aligned}
$$

omitting the center of mass motion and $H_{\text {rel }}$. The coordinates of the electrons now refer to the center of mass. The Hamiltonian has been divided into an electronic part $H_{\mathrm{e}}$, and a nuclear part $H_{\mathrm{n}}$, where $H_{n}$ is simply

$$
H_{n}=-\frac{1}{2 \mu} \nabla^{2}(r, \theta, \phi) \text {. }
$$


As $H_{\mathrm{n}}$ does not contain any electronic coordinates, we consider the following ansatz

$$
\Psi=\psi_{\mathrm{e}}(r, \mathbf{x}) \psi_{\mathrm{n}}(r, \theta, \phi)
$$

The electrostatic energy depends on $r$, therefore $\psi_{e}=\psi_{e}(r, \mathbf{x})$. We assume that $\psi_{\mathrm{e}}(r, \mathbf{x})$ is a solution of the electronic part of the Schrödinger equation for a fixed value of $r$, hence

$$
H_{e} \psi_{e}(r, \mathbf{x})=E_{e}(r) \psi_{e}(r, \mathbf{x})
$$

By using Eqs. (2.4) and (2.6) in the time-independent Schrödinger equation (2.2) we obtain

$$
\left(H_{e}+H_{n}\right) \psi_{e}(r, \mathbf{x}) \psi_{n}(r, \theta, \phi)=E \psi_{e}(r, \mathbf{x}) \psi_{n}(r, \theta, \phi)
$$

We find by multiplication with $\psi_{e}^{*}(r, \mathbf{x})$ from the left and integration over the electron coordinates that

$$
\left\langle\psi_{e}\left|H_{n}\right| \psi_{e}\right\rangle \psi_{n}(r, \theta, \phi)+E_{e}(r) \psi_{n}(r, \theta, \phi)=E \psi_{n}(r, \theta, \phi) .
$$

In the Born-Oppenheimer approximation it is assumed that $\left\langle\psi_{e}\left|H_{n}\right| \psi_{e}\right\rangle=$ $H_{n}$, i.e. the dependency of $r$ in this integral is omitted and,

$$
H_{n} \psi_{n}(r, \theta, \phi)+E_{e}(r) \psi_{n}(r, \theta, \phi)=E \psi_{n}(r, \theta, \phi) .
$$

As it turns out most of the terms omitted can be included in $E_{e}(r)$. This is the adiabatic Born-Oppenheimer approximation.

To summarize, the energy $E$ of a diatomic molecule is then given by

$$
E=E_{\text {translational }}+E_{\text {electronic }}+E_{\text {nuclei }}
$$

and we will find that $E_{\text {nuclei }}$ can be further separated into a vibrational and a rotational contribution. The translational energy is not included in Eq. $(2.10)$.

Finally we comment that the electronic functions $\psi_{e}(r, \mathbf{x})$ are classified according to the eigenvalue $\Lambda$ of the $L_{z}$ operator, i.e. the angular momentum along the molecule fixed axis. In the present work we have considered the electronic functions $\psi_{e}(r, \mathbf{x})$ corresponding to two ground state alkali atoms ( ${ }^{2} S$ atoms). The only possible value of $\Lambda$ is then zero. Generally, spectroscopic notation is introduced to specify the value of $\Lambda$ for molecules. A few examples are: $\Sigma(\Lambda=0), \Pi(\Lambda=1), \Delta(\Lambda=2), \varphi(\Lambda=3), \ldots$ 


\subsection{Born-Oppenheimer potential curves}

From Eqs. (2.7) and (2.10) the functions $E_{e}(r)$ are seen to be important for quantitative calculations. In the present work we will be concerned with atom-atom collisions (chapter 5 ) that require the interatomic potentials $E_{e}(r)$ to be known with high accuracy. We will in the following refer to the functions $E_{e}(r)$ as potential curves. See Fig. (5.5) for examples of two potential curves.

It is perhaps not obvious, but Eq. (2.7) represents a very challenging many-body problem. This is due to the mutual Coulomb repulsion between the electrons. With many electrons on both nuclei these terms become even more important.

The problem in the present context is to obtain the function $E_{e}(r)$ and the many-body wave function $\psi_{e}(r, \mathbf{x})$, from which additional properties of interest may be computed.

Hartree proposed to write the wave function $\psi_{e}$ as a product of singleparticle wave functions

$$
\psi_{e}\left(\mathbf{r}_{1}, \mathbf{r}_{2}, \ldots, \mathbf{r}_{N}\right)=\psi_{a}\left(\mathbf{r}_{1}\right) \psi_{b}\left(\mathbf{r}_{2}\right) \ldots \psi_{x}\left(\mathbf{r}_{N}\right),
$$

where the one-particle functions $\psi_{a}\left(\mathbf{r}_{i}\right)$ satisfies

$$
\left[-\frac{1}{2} \nabla^{2}\left(\mathbf{r}_{i}\right)+V_{n}+U_{a}\left(\mathbf{r}_{i}\right)\right] \psi_{a}\left(\mathbf{r}_{i}\right)=\epsilon_{a} \psi_{a}\left(\mathbf{r}_{i}\right) .
$$

The one particle energies are denoted $\epsilon_{a}$. The potential $V_{n}$ includes the electrostatic interactions with the nuclei, whereas $U_{a}\left(\mathbf{r}_{i}\right)$ is given by

$$
U_{a}\left(\mathbf{r}_{i}\right)=\sum_{b \neq a} \int d^{3} r^{\prime} \frac{\psi_{b}^{*}\left(\mathbf{r}^{\prime}\right) \psi_{b}\left(\mathbf{r}^{\prime}\right)}{\left|\mathbf{r}_{i}-\mathbf{r}^{\prime}\right|} .
$$

The labels $a, b, \ldots, x$ denote the one-particle quantum numbers and the oneparticle functions $\psi_{a}\left(\mathbf{r}_{i}\right)$ include the electronic spin. Notice that Eq. (2.13) really is a set of coupled non-linear differential equations. The solutions can only be found numerically through an iterative process. The potential $U_{a}\left(\mathbf{r}_{i}\right)$ represents the effect of the electron-electron interactions on the electron in orbital $\psi_{a}\left(\mathbf{r}_{i}\right)$, created by the charge density

$$
\rho\left(\mathbf{r}^{\prime}\right)=-\sum_{b \neq a}\left|\psi_{b}\left(\mathbf{r}^{\prime}\right)\right|^{2} .
$$

The wave function in Eq. (2.12) does not satisfy the Pauli exclusion principle. This can be rectified by using a Slater determinant for $\psi_{e}$ in place of Eq. (2.12). An additional non-local term is then introduced in 
the Schrödinger equation, but the accuracy of the solutions and energies is improved. In the Hartree-Fock (HF) method one seeks the Slater determinant $\Phi$ that minimizes the expectation value

$$
E_{\mathrm{HF}}=\left\langle\Phi\left|H_{e}\right| \Phi\right\rangle
$$

The HF method plays an important role in quantum chemistry and is often used as a starting point for other more refined methods. We will not describe any such post HF method in detail. However, we comment on the MøllerPlesset many-body perturbation theory as it is used in two of the papers included in this thesis (papers I and IV).

\subsubsection{Comments on the Møller-Plesset perturbation theory}

Basically Møller-Plesset perturbation theory is Rayleigh-Scrödinger perturbation theory applied to the perturbation $H_{e}-H_{\mathrm{HF}}$, with the HF Hamiltonian as the zero order Hamiltonian. The energy difference between the energy $E_{\mathrm{HF}}$ and the exact energy is often called electron-correlation energy [12].

The zero-order Hamiltonian $H^{(0)}$ is the HF Hamiltonian that fulfills

$$
H_{\mathrm{HF}} \Phi_{0}=E_{0}^{(0)} \Phi_{0}
$$

where $\Phi_{0}$ is the HF ground-state wave function. This choice of $H_{0}$ was made by C. Møller and M. S. Plesset. [13]. The perturbation $H^{(1)}$ is then given by

$$
H^{(1)}=H_{e}-H_{\mathrm{HF}},
$$

and the first order energy correction is

$$
E_{0}^{(1)}=\left\langle\Phi_{0}\left|H^{(1)}\right| \Phi_{0}\right\rangle .
$$

However, the energy $E_{\mathrm{HF}}$ is obtained as the expectation value $E_{\mathrm{HF}}=\left\langle\Phi_{0}\left|H_{e}\right| \Phi_{0}\right\rangle$ and we see that

$$
E_{\mathrm{HF}}=E_{0}^{(0)}+E_{0}^{(1)}
$$

The correction to the Hartree-Fock energy comes from second order perturbation theory along with higher order terms when included. The second order correction is given by

$$
E^{(2)}=\sum_{i \neq 0} \frac{\left\langle\Phi_{i}\left|H^{(1)}\right| \Phi_{0}\right\rangle\left\langle\Phi_{0}\left|H^{(1)}\right| \Phi_{i}\right\rangle}{E_{0}^{(0)}-E_{i}^{(0)}},
$$


where $\Phi_{i}$ is an eigenfunction of $H_{\mathrm{HF}}$ with the eigenvalue $E_{i}^{(0)}$. The evaluation and implementation of Eq. (2.21) have not been part of the present work. We have used the quantum chemistry software GAMESS [14] and the implementation of MP2 $[15,16]$ distributed with it.

Møller-Plesset perturbation theory was chosen as the post Hartree-Fock method in the present work. We believe that it combines accuracy with a reasonable computational effort. However, we did not consider the MP2 energies as highly accurate. This was made clear in both papers where results that relied on MP2 energies were reported. In paper IV we quantified the uncertainties in the MP2 energies to be $\pm 20 \%$.

\subsubsection{Basis sets}

In Eq. (2.12) $\psi_{a}(\mathbf{r})$ denotes molecular orbitals given by

$$
\psi_{a}(\mathbf{r})=\sum_{\alpha=1}^{M} c_{a \alpha} \phi_{\alpha}(\mathbf{r}),
$$

where $\phi_{\alpha}$ are Gaussian functions of the form (written in Cartesian coordinates)

$$
\phi(\mathbf{r})=x^{i} y^{j} z^{k} e^{-k r^{2}},
$$

and $c_{a \alpha}$ are constants. On physical grounds, what is known as Slater orbitals ${ }^{1}$, may be preferable to the Gaussian functions in Eq. (2.23). However, the two center integrals involved in the HF calculations are very cumbersome to evaluate with Slater orbitals. Two center integrals can be handled much more efficiently with Gaussian orbitals.

Contracted Gaussian functions are often used. A contracted Gaussian function is a fixed linear combination of Gaussian functions, centered at the same atomic nucleus. The advantage is less coefficients to determine. The linear combination should however be carefully chosen to avoid loss of accuracy.

The number of Gaussian functions to include in a calculation is chosen as a compromise between accuracy and efficiency. Minimal basis sets, which only include one function for each orbital, may be used. A minimal basis set for hydrogen may include one function to represent the $1 s$ orbital. This is

\footnotetext{
${ }^{1}$ The Slater orbitals are given by

$$
\phi(\mathbf{r})=r^{n-1} e^{-k r} Y_{l m_{l}}(\theta, \phi)\{\uparrow, \downarrow\},
$$
}

where $n, l, m_{l}$ and $\uparrow \downarrow$ are one-electron quantum numbers, and $k$ is a constant. 
certainly inadequate but very efficient. More involved is the "double zeta" basis sets, in which two functions are used for each orbital, or even the "triple zeta" basis sets with three functions for each orbital.

The basis sets used in the present work have included polarization functions to account for the distortion of the orbitals by molecular bonds. The quantum chemistry package GAMESS has an extensive library of basis sets. Generally we have used rather large basis sets, but more details can be found in the respective papers.

\subsubsection{Potential curves at long range}

At large internuclear separations the interatomic potential, i.e. the potential curve, between two $S$ state atoms is given by the analytical expression [8]

$$
E_{e}(r)=V(r)=-\frac{C_{6}}{r^{6}}-\frac{C_{8}}{r^{8}}-\frac{C_{10}}{r^{10}} .
$$

The coefficients $C_{6}, C_{8}$ and $C_{10}$ are called dispersion coefficients and the attractive forces acting between two atoms far apart, due to the potential $V(r)$, are called van der Waals forces.

\subsection{Vibration and rotation}

Finally we return to Eq. (2.10) and study the wave functions $\psi_{n}(r, \theta, \phi)$ in more detail. We may write the Hamiltonian in Eq. (2.5) in the form

$$
H_{n}=-\frac{1}{2 \mu} \frac{1}{r^{2}} \frac{\partial}{\partial r}\left(r^{2} \frac{\partial}{\partial r}\right)+\frac{\mathbf{R}^{2}}{2 \mu^{2} r^{2}}+E_{e}(r),
$$

where we have introduced $\mathbf{R}$ as the angular momentum of the relative motion of the nuclei. The Schrödinger equation (2.10) simplifies by using the ansatz

$$
\psi_{n}(r, \theta, \phi)=\frac{1}{r} \psi_{v R}(r) \psi_{R}(\theta, \phi),
$$

where $\psi_{R}(\theta, \phi)$ are eigenfunctions for the $\mathbf{R}^{2}$ operator. We then obtain the result

$$
\left(-\frac{1}{2 \mu} \frac{d^{2}}{d r^{2}}+\frac{R(R+1)}{2 \mu r^{2}}+E_{e}(r)\right) \psi_{v R}(r)=E \psi_{v R}(r) .
$$

The wave functions $\psi_{v R}(r)$ are the ro-vibrational wave functions. They depend on the vibrational quantum number $v$, and the rotational quantum 
number $R$. This is a rather complicated differential equation, which can not be solved exact without additional approximations, in particular since $E_{e}(r)$ is unknown.

Previously, upon deriving Eq. (2.10), we neglected the $r$ dependence of $\psi_{e}$ and wrote $\left\langle\psi_{e}\left|H_{n}\right| \psi_{e}\right\rangle=H_{n}$. We may now include parts of this previously omitted contribution and reconsider the matrix element

$$
\frac{1}{2 \mu r^{2}}\left\langle\psi_{e}\left|\mathbf{R}^{2}\right| \psi_{e}\right\rangle \psi_{n}
$$

i.e we still neglect the contribution from the first part of $H_{n}$ (cf. Eq. (2.26)) . To work out the matrix element in Eq. (2.29) we introduce the total angular momentum of the molecule, excluding electronic and nuclear spin,

$$
\mathbf{N}=\mathbf{R}+\mathbf{L} .
$$

We note that $\mathbf{R}^{2}=\mathbf{N}^{2}-2 \mathbf{N} \cdot \mathbf{L}+\mathbf{L}^{2}$, and that $\Psi=\psi_{e} \psi_{n}$ are eigenfunctions for ${ }^{2} \mathbf{N}^{2}$ and $N_{z}$

$$
\begin{aligned}
\mathbf{N}^{2} \Psi & =N(N+1) \Psi, \\
N_{z} \Psi & =M_{N} \Psi,
\end{aligned}
$$

where $N_{z}$ is the component of $\mathbf{N}$ along the space-fixed $z$-axis. The matrix element in Eq. (2.29) can then be written

$$
\begin{aligned}
\frac{1}{2 \mu r^{2}}\left\langle\psi_{e}\right| \mathbf{N}^{2}- & 2 \mathbf{N} \cdot \mathbf{L}+\mathbf{L}^{2}\left|\psi_{e}\right\rangle \psi_{n}= \\
& \frac{1}{2 \mu r^{2}}\left(N(N+1)-2\left\langle\psi_{e}|\mathbf{N} \cdot \mathbf{L}| \psi_{e}\right\rangle+\left\langle\psi_{e}\left|\mathbf{L}^{2}\right| \psi_{e}\right\rangle\right) \psi_{n}
\end{aligned}
$$

The term proportional to $\mathbf{L}^{2}$ can be included in the electronic and vibrational energies, i.e. the contribution from this term is independent of the rotational quantum number $N$. The term proportional to $\mathbf{N} \cdot \mathbf{L}$ gives the so called $\Lambda$ splitting that will be omitted.

We may then write for the relative motion of nuclei A and B,

$$
\left(-\frac{1}{2 \mu} \frac{d^{2}}{d r^{2}}+\frac{N(N+1)}{2 \mu r^{2}}+E_{e}(r)\right) \psi_{v N}(r)=E \psi_{v N}(r),
$$

and the complete wave function for the nuclei $\psi_{n}(r, \theta, \phi)$ is

$$
\psi_{n}(r, \theta, \phi)=\frac{1}{r} \psi_{v N}(r) \psi_{N M_{N} \Lambda}(\theta, \phi),
$$

\footnotetext{
${ }^{2}$ This means that $\psi_{n}$ is redefined and that Eq. (2.27) is no longer valid. See also Eq. (2.35).
} 
where $\psi_{N M_{N} \Lambda}(\theta, \phi)$ is the rotational wave function of a diatomic molecule with total angular momentum $\mathbf{N}$ for a given value of $\Lambda$. The eigenvalue $E$ depends on the rotational quantum number $N$, the vibrational quantum number $v$ and the electronic state $\psi_{e}(r, \mathbf{x})$.

With some approximations we can write the total energy (not included the translational energy) as

$$
E=E_{\mathrm{el}}(r)+E_{\mathrm{vib}}+E_{\mathrm{rot}}=E_{\mathrm{el}}(r)+E_{\mathrm{vib}}+\frac{1}{2 \mu}\left\langle\psi_{v}(r)\left|\frac{1}{r^{2}}\right| \psi_{v}(r)\right\rangle N(N+1),
$$

where $E_{\text {vib }}$ and $\psi_{v}(r)$ are obtained by setting $N=0$ in Eq. (2.34) and solving

$$
-\frac{1}{2 \mu} \frac{d^{2}}{d r^{2}} \psi_{v}(r)+E_{e}(r) \psi_{v}(r)=E_{\mathrm{vib}} \psi_{v}(r) .
$$

The vibrational energies $E_{\mathrm{vib}}$, computed for the ground electronic state of ${ }^{6} \mathrm{Li}_{2}$, are shown in Fig. 2.2.

\subsection{Cold and ultracold molecules}

In chapter 1 we described methods to cool and trap a gas of atoms. The next natural step is to attempt to cool gases that consist of diatomic molecules. In the previous section we discussed the electronic, vibrational and rotational states of such particles. We found that the inner structure was indeed far more complex than for atoms, with several additional degrees of freedom.

The quest for cold and ultracold diatomic molecules is due to quantum mechanics, and in particular the wave-particle duality of matter. When the de Broglie wavelength

$$
\lambda_{\mathrm{dB}}=\frac{1}{\sqrt{2 m \pi k_{B} T}},
$$

for a particle of mass $m$ at a temperature $T$ becomes large, in a sense the size of the particles increases. In this regime it is not adequate to think of particles as hard spheres, as their wavelike properties become important. When the inter particle separation becomes less than the de Broglie wavelength, a quantum mechanical description must be used.

Spectacular physical phenomena have been observed in this regime. For example molecular Bose-Einstein condensates [18, 19] and the crossover between a Bose-Einstein condensate and the Bardeen-Cooper-Schrieffer (BCS) state $[20,21]$ with de-localized pairing of (fermionic) atoms. 


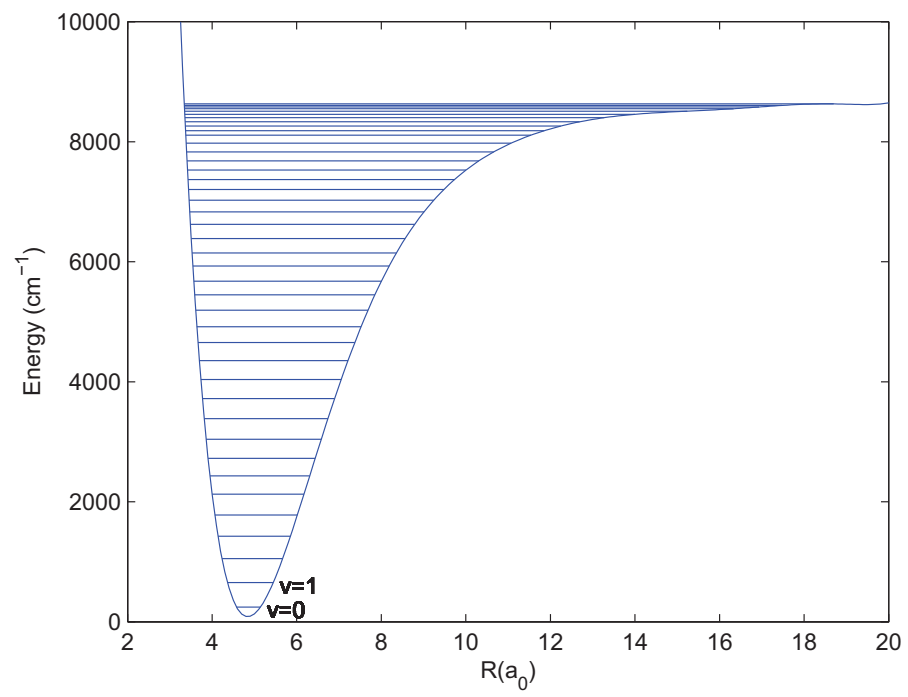

Figure 2.2: Vibrational energies in the electronic ground state of ${ }^{6} \mathrm{Li}_{2}$, computed from Eq. (2.37). The vibrational levels $v=0$ and $v=1$ are explicitly labeled. The 41 lowest vibrational levels are shown. The figure was made by the present author to be included in a different work [17], hence, $R$ is used in place of $r$.

Cold and ultracold molecules are also well suited for high precision spectroscopy. Their low kinetic energy offer longer observation time. Highly precise measurements can even be used to investigate the variation of fundamental constants, such as the fine structure constant and the electron-toproton mass ratio [22].

It has even been suggested that ultracold polar molecules may provide the qubits for a quantum computer [23]. Although the quantum computer is far from being realized, ultracold molecules are also relevant for the field of quantum information theory.

Finally, cold atoms and molecules may be used in atomic and molecular interferometers. The interferometer may be used as an instrument for precise measurements of e.g. the gravitational constant or as a demonstration of the fundamentals of quantum mechanics.

Motivated by the possible applications we now discuss how cold and ul- 
tracold molecules can be created. This topic is revisited in chapters 4 and 5.

\subsubsection{Obtaining cold molecules}

Is there a method that can be used to cool a gas of any molecular species to very low temperatures? In general the answer is no. We will limit the discussion to neutral diatomic molecules, and we do not discuss methods that only apply to polar molecules.

There are then two different strategies: Direct or indirect cooling. In the direct cooling methods the kinetic energy is removed from the molecules by collisions, or by some other convenient mechanism. Indirect cooling methods are based on cold atoms together with a bonding mechanism that allows the two cold atoms to form a cold diatomic molecule.

Laser cooling was a great success when applied to alkali atoms. It may therefore seem natural to attempt laser cooling of diatomic molecules. However, the complex ro-vibrational structure makes it hard to obtain the closed cycle necessary. Ideally, absorption of a photon should be followed by almost instantaneous spontaneous emission and the molecule should return to the initial state (see Fig. 2.3). However, the problem with diatomic molecules is that the spontaneous emission populates many different ro-vibrational levels, and a significant fraction of the initial molecules is thereby lost.
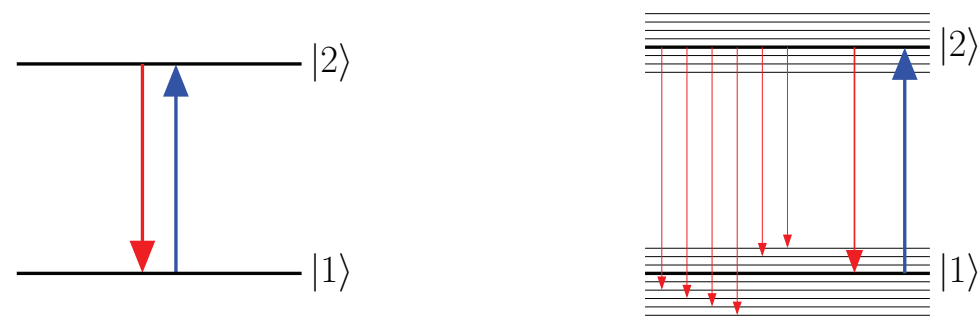

Figure 2.3: The left panel shows a closed two level cycle between the states $|1\rangle$ and $|2\rangle$, typical for alkali atoms. In the right panel the more complex inner structure of a molecule is shown schematically. A laser (blue arrow) is used to excite the molecule to the electronic state $|2\rangle$. By spontaneous emission the molecule returns to one of the ro-vibrational levels of the electronic state $|1\rangle$. Molecules populating ro-vibrational states different from the initial state are not in resonance with the laser, and can not be cooled further.

In the next section we present two selected and successful cooling methods 
used to cool diatomic molecules.

\subsubsection{Photoassociation (PA) of diatomic molecules}

Two colliding atoms and a photon with the proper energy can create a molecule in an excited electronic state. In short this is the essence of photoassociation, an example of an indirect cooling method. Two free ground state atoms, cooled to microkelvin temperatures, approach each other with an energy corresponding to the electronic molecular ground state. By absorbing a photon the two atoms are excited to a bound vibrational level of an excited molecular electronic state. The process is schematically shown in Fig. 2.4. Two ultracold atoms remain most of the time far apart. Thus, even though

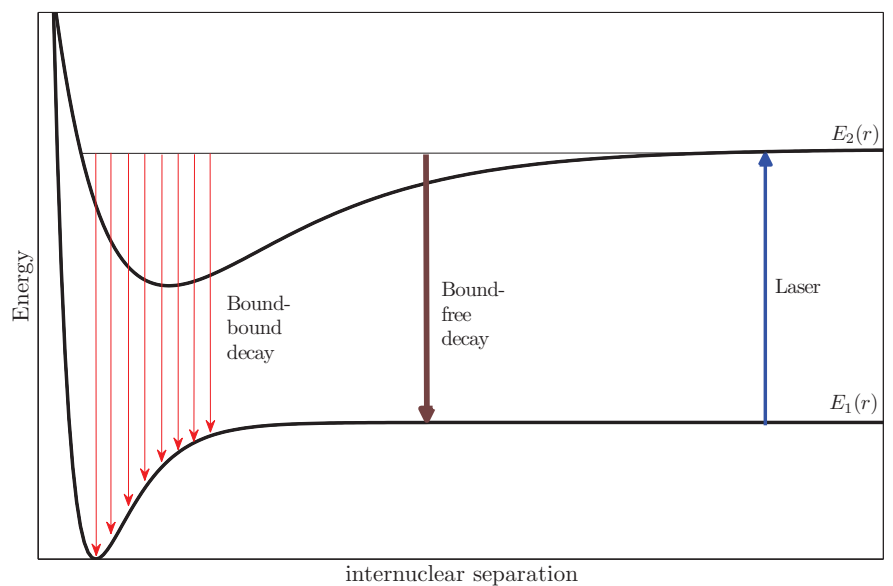

Figure 2.4: Illustration of the PA process and the spontaneous decay to the bound ro-vibrational levels in the electronic ground state $E_{1}(r)$, as well as the decay to the continuum limit. A photon (blue arrow) excites the two ground state atoms to a bound vibrational level of an excited electronic state $E_{2}(r)$. Molecules can decay to the ground state, either to a bound ro-vibrational level or to the continuum of levels, i.e to two free ground state atoms.

the two atoms are excited by a photon, they are not always excited to a bound molecular level. 
A simple argument can be used to estimate the strength of the boundbound decay. The transition from a bound vibrational level in the excited electronic state, $\psi_{v^{\prime}}$, to a vibrational level in the ground state, $\psi_{v^{\prime \prime}}$, happens almost instantaneously. During this short period of time the internuclear separation is unchanged. Referring to Fig. 2.4, this means that the spontaneous decay is vertical, as indicated by the red arrows. Generally the equilibrium distance $r_{e}$ is larger for the excited states. When the excited electronic state has an equilibrium distance significantly larger than that of the ground state, the overlap between the wave functions $\psi_{v^{\prime}}$ and $\psi_{v^{\prime \prime}}$ is small, and the probability for bound-bound decay is low. Clearly this is not a favorable situation for cold molecule production. Different techniques have been proposed to enhance the fraction of the bound-bound decay, see [24] and references therein. Still it is a challenge to enable both efficient production of molecules and simultaneously create deeply bound ground state molecules.

Cold and ultracold molecules have been produced by the use of PA. This includes homonuclear diatomic molecules of the alkalies $\mathrm{Li}, \mathrm{Na}, \mathrm{K}, \mathrm{Rb}$ and $\mathrm{Cs}$, but also heteronuclear diatomic molecules composed of alkali atoms. Examples include KRb, RbCs and NaCs. References can be found in [24]. The temperatures of these molecules vary between millikelvin and microkelvin with densities in the range of $10^{3}-10^{6} \mathrm{~cm}^{-3}$.

Although PA is a successful method it can only be used to create molecules from atoms cooled by one of the more traditional methods. This excludes among others the hybrides and the oxides.

\subsubsection{Play pool to stay cool}

A direct cooling method that resembles a game of pool has been proposed and demonstrated for NO molecules by Elioff et al. [25]. The detailed reaction can be expressed

$$
\mathrm{NO}+\mathrm{Ar} \rightarrow \mathrm{NO}^{\prime}+\mathrm{Ar}
$$

The NO molecules are cooled in one collision with Ar in a crossed molecular beam experiment (see Fig. 2.5). The result is NO molecules in an excited rotational state, indicated with the notation $\mathrm{NO}^{\prime}$ in Eq. (2.39). Elliot et al. claim this method is general as it does not rely on any particular property of the molecule. Strangely we have not seen any articles describing the use of this method on diatomic molecules besides NO.

Consider the collision of a NO molecule with an Ar atom. In the laboratory system the NO molecule has the velocity $\mathbf{v}$, while the center of mass system has velocity $\mathbf{v}_{\mathrm{cm}}$ in the same system. In the center of mass system 
the NO molecule has velocity $\mathbf{u}$ before the collision. Post collision velocities are primed. The collision between Ar and NO should be such that the velocity of the NO molecule is zero after the collision, i.e. $\mathbf{v}^{\prime}=0$. Expressed mathematically as

$$
\mathbf{v}^{\prime}=\mathbf{u}^{\prime}+\mathbf{v}_{\mathrm{cm}}=\mathbf{0}
$$

This requirement can be used to obtain the scattering angle $\theta_{C M}$ [25]

$$
\cos \theta_{\mathrm{CM}}=\frac{E_{\mathrm{Ar}}-E_{\mathrm{NO}}}{\sqrt{\left(E_{\mathrm{NO}}+\frac{M}{m} E_{\mathrm{Ar}}\right)\left(E_{\mathrm{NO}}+\frac{m}{M} E_{\mathrm{Ar}}\right)}},
$$

with $E_{\mathrm{Ar}}$ and $E_{\mathrm{NO}}$ as the kinetic energies of the Ar atom and the NO molecule respectively, before the collision. We see from Eq. (2.39) that the NO molecules are excited after the collision. A fraction of the collision energy is transformed to internal energy of the NO molecule. The internal energy gained in the collision is [25]

$$
E_{\text {internal }}=\left(1-\frac{M}{m}\right) E_{\mathrm{NO}}
$$

The temperature at a density of $10^{8}$ molecules per cubic centimeter was measured to be $\simeq 440 \mathrm{mK}$, reported in [25]. Five years later, the method was improved and the reported temperature was $35 \mathrm{mK}$, with a significantly prolonged observation time [26].

\subsection{Further reading}

The presentation of the Born-Oppenheimer approximation is based on a local compendium on diatomic molecules [27], along with the book "Physics of Atoms and Molecules" by Bransden and Joachain [28]. The sections on photoassociation and billiard cooling are inspired by the presentations given in the cited articles. More information about photoassociation (including references) can be found in [17]. 


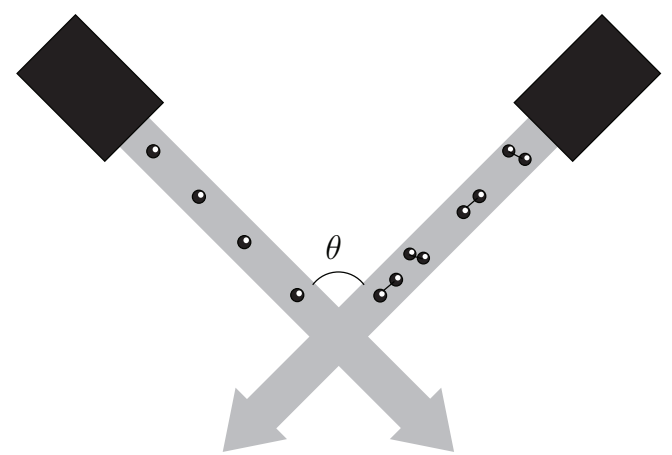

Figure 2.5: Sketch of the crossed beams in the pool like cooling method. Collisions between Ar atoms and NO molecules occur at the intersection of the two beams. The rotational degree of freedom of the NO molecule is excited and its kinetic energy is close to zero after the collision. 



\section{Chapter 3}

\section{Cold collisions}

In previous chapters we have seen that collisions play an important role in cold and ultracold atomic and molecular physics. We have occasionally referred to results from scattering theory in both chapters 1 and 2. The pool cooling method also relied on collisions.

In this chapter we will introduce atomic and molecular scattering theory. We restrict ourselves to cold atom - atom and atom - molecule collisions, using diatomic molecules. The classical picture of a collision is that of two

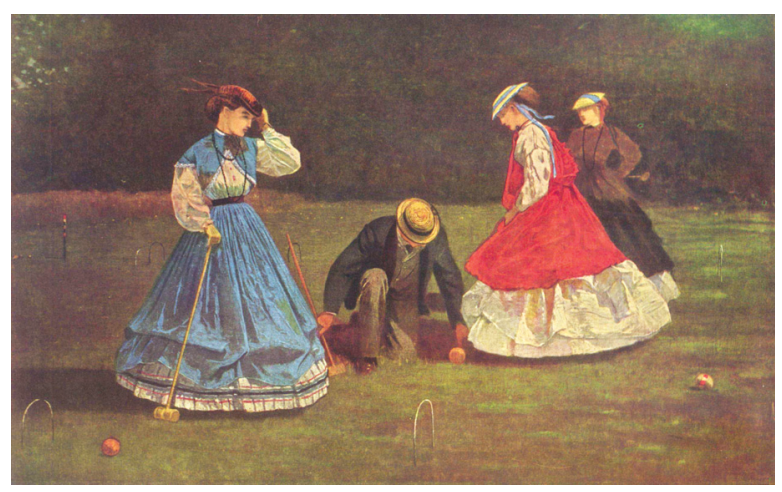

Figure 3.1: (Winslow Homer: Croquet, 1864) Croquet is an example of a game of classical hard sphere collisions. In quantum mechanics, this classical view of collisions is no longer sufficient.

hard spheres colliding, as experienced in pool or croquet. Although the hard sphere collisions represent a useful mental picture, quantum mechanics requires that we represent the particles with wave functions satisfying the 
Schrödinger equation.

We begin this chapter by introducing one-channel elastic scattering and define both the scattering length and the phase shift, as well as the scattering amplitude and the cross section. The theory of inelastic scattering and the multichannel equations are then introduced and the close-coupled equations are obtained. Finally we introduce the scattering matrix (the $S$-matrix).

\subsection{Elastic scattering}

Elastic scattering means that only kinetic energy is exchanged between the colliding particles, i.e. the internal states do not change in a collision.

The time-independent Schrödinger equation reads

$$
H \Psi(\mathbf{r})=E \Psi(\mathbf{r}),
$$

and the scattering wave functions $\Psi$ correspond to energies $E>0$ in the present chapter. A particle of definite energy $E=\frac{k^{2}}{2 m}$ and mass $m$ traveling along the $z$-axis is described by the wave function

$$
\psi(z)=e^{i k z} .
$$

After a collision the particle is represented as a spherical wave of the form (see Fig. 3.2)

$$
\psi(r)=\frac{e^{i k r}}{r} .
$$

The wave function in Eq. (3.3) assumes that the particle is moving with

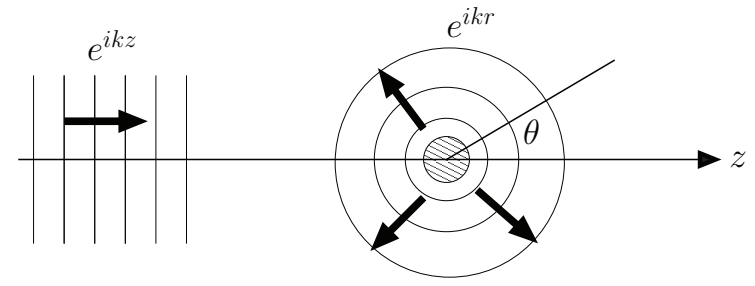

Figure 3.2: Incoming plane wave along the $z$-axis along with an outgoing spherical wave.

the same probability in all directions. The extra factor of $1 / r$ is included 
to ensure that probability is conserved [29]. To allow for scattering with different probabilities in different directions $\theta$, the scattering amplitude $f(\theta)$ is introduced and Eq. (3.3) is modified

$$
\psi(r)=\frac{f(\theta) e^{i k r}}{r}
$$

In the region where the scattering potential $V$ is zero we seek a wave function of the form

$$
\Psi(r, \theta)=A\left(e^{i k z}+\frac{f(\theta) e^{i k r}}{r}\right),
$$

where $A$ is an undetermined constant. We will in this section consider spherical symmetric potentials, $V=V(r)$. The Schrödinger equation then reads

$$
\left(-\frac{1}{2 \mu} \nabla^{2}(\mathbf{r})+V(r)\right) \Psi(\mathbf{r})=E \Psi(\mathbf{r})
$$

where $\mu$ is the reduced mass of the system and

$$
\nabla^{2}=\frac{\partial^{2}}{\partial r^{2}}+\frac{2}{r} \frac{\partial}{\partial r}+\frac{\mathbf{L}^{2}}{r^{2}}
$$

The orbital angular momentum operator is $\mathbf{L}^{2}$. The wave function in Eq. (3.5) represents a solution to the Shcrödinger equation in the region where $r \rightarrow \infty$ and $V=0$.

We also need to find the wave function in the interacting region $(V \neq 0)$. It is common to introduce the notation

$$
k^{2}=2 \mu E, \quad U(r)=2 \mu V(r),
$$

to write Eq. (3.6) in the form

$$
\left(\nabla^{2}(\mathbf{r})+k^{2}-U(r)\right) \Psi(\mathbf{r})=0 .
$$

We use the partial wave expansion to expand the wave function $\Psi(\mathbf{r})$ in terms of the Legendre polynomials $P_{l}(\cos \theta)$

$$
\Psi(r, \theta)=\frac{1}{r} \sum_{l=0}^{\infty} a_{l} \psi_{l}(r) P_{l}(\cos \theta),
$$

where the expansion coefficients $a_{l}$ are introduced. The incoming wave breaks the spherical symmetry (therefore we include $\theta$ ), but the azimuthal symmetry remains and justifies that $\Psi$ can not depend on the angle $\phi$. By combining 
Eqs. (3.9) and (3.10) we find the radial Schrödinger equation that $\psi_{l}(r)$ fulfills

$$
\left(\frac{d^{2} \psi_{l}(r)}{d r^{2}}+k^{2}-U(r)-\frac{l(l+1)}{r^{2}}\right) \psi_{l}(r)=0 .
$$

The spherical symmetric potential allows for solutions of the form $\Psi(\mathbf{r})=$ $\psi_{l}(r) Y_{l m_{l}}(\theta, \phi)$, but since $\Psi$ does not depend on $\phi, m=0$ and $\Psi(\mathbf{r})=$ $\psi_{l}(r) Y_{l 0}(\theta, \phi) \propto \psi_{l}(r) P_{l}(\cos \theta)$.

The radial equation (3.11) motivates the definition of an effective potential

$$
U_{l}(r)=U(r)+\frac{l(l+1)}{r^{2}} .
$$

In atom-atom collisions the interatomic potential $V(r)$ may be taken as the Born-Oppenheimer potential $E_{e}(r)$, introduced in the previous chapter. The effective potentials for the quantum numbers $l=0,1,2,3$ are plotted in Fig. 3.3. The partial wave quantum number $l$ represents the quantized angular momentum for the relative motion of the colliding particles. The function $\psi_{l}(r)$ in Eq. (3.10) is often referred to as partial wave $l$.

At low temperatures the smallest values of $l$ are by far the most important in expansion (3.10). Due to the low energy involved in the collisions together with the repulsive centrifugal barrier $\frac{l(l+1)}{r^{2}}$, only the $l=0$ partial wave is able to penetrate into the region where $U(r) \neq 0$ (see Fig. 3.3). In the ultracold regime it is therefore sufficient to include only the term with $l=0$ in Eq. (3.10), this is called $s$-wave scattering or $s$-wave collisions.

The solutions to Eq. (3.11) can only be obtained numerically, except for a few very special potentials. Numerovs method, described in Numerical Recipes [30], may be well suited for this task.

If $U(r)=0$ in Eq. (3.11) the general solution, which may be found in almost any quantum mechanics textbook, is

$$
\psi_{l}(r)=A r j_{l}(k r)+B r n_{l}(k r),
$$

where $j_{l}(k r)$ and $n_{l}(k r)$ are the spherical Bessel functions. To facilitate comparison with the wave function in Eq. (3.5) (valid in the region where $U_{l}(r)$ is zero) it is more convenient to work with spherical Hankel functions. In terms of the Bessel functions introduced in Eq. (3.13) the first and second spherical Hankel functions, $h_{l}(x)$ and $h_{l}^{(2)}(x)$ respectively, are written

$$
\begin{aligned}
h_{l}(x) & =j_{l}(x)+i n_{l}(x), \\
h_{l}^{(2)}(x) & =j_{l}(x)-i n_{l}(x) .
\end{aligned}
$$




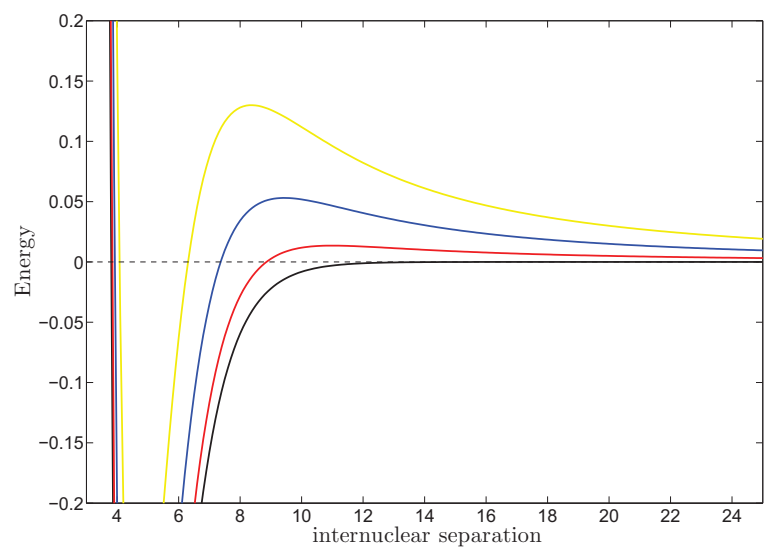

Figure 3.3: The effective potential $U_{l}(r)=U(r)+\frac{l(l+1)}{r^{2}}$ for $l=0,1,2$ and 3 . The yellow line corresponds to $l=3$, the blue line correspond to $l=2$, the red line corresponds to $l=1$ and the black line corresponds to $l=0$. The interatomic potential $U(r)$ is assumed to be rather shallow to emphasize the effect of the repulsive centrifugal term. Arbitrary units are used.

For large values of $x$ the first spherical Hankel function $h_{l}(x)$ is $h_{l}(x)=$ $(-i)^{l+1} e^{i k x} / x$, which is what we want (apart from $(i)^{l+1}$ ), cf. Eq. (3.5). In addition, it also satisfies the Schrödinger equation in the intermediate region. The different scattering regions are illustrated in Fig. 3.4.

Further, we rewrite the wave function in Eq. (3.10) in the convenient form

$$
\Psi(r, \theta)=A\left(e^{i k z}+k \sum_{l=0}^{\infty} i^{l+1}(2 l+1) a_{l} h_{l}(k r) P_{l}(\cos \theta)\right) .
$$

where the coefficients $a_{l}$ have been redefined as $a_{l} \rightarrow k i^{l+1}(2 l+1) a_{l}$. This is only for convenience, as we will see. We have explicitly written $e^{i k z}$ as the wave function for the incoming wave, whereas the second term represents an outgoing spherical wave. By comparing Eqs. (3.16) and (3.5) the scattering amplitude is obtained

$$
f(\theta)=\sum_{l=0}^{\infty}(2 l+1) a_{l} P_{l}(\cos \theta)
$$




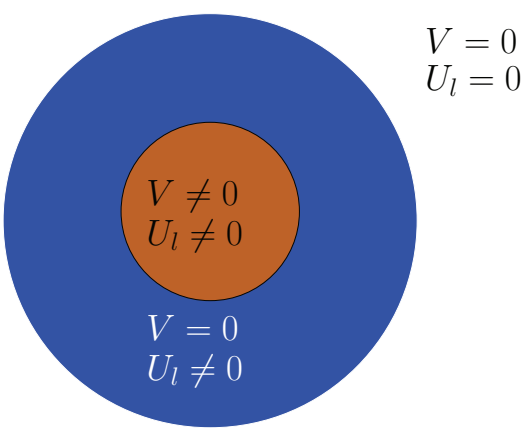

Figure 3.4: The brown circle represents the scattering region where the full Schrödinger equation (3.11) is solved. The blue circle represents the intermediate region where the potential $V(r)$ is zero, but the centrifugal term $l(l+1) / r^{2}$ is non-zero. The asymptotic region (also called the radiation zone) is characterized by $U=V=U_{l}=0$, and the solution to the Schrödinger equation in this region is given by Eq. (3.5).

The scattering cross section is defined as the ratio of the scattered flux per unit solid angle $d \Omega$ to the incident flux per unit area, in analogy with the classical definition found in e.g. Classical Mechanics [31]

$$
\frac{d \sigma(\theta)}{d \Omega}=\frac{r^{2}\left|\frac{f(\theta) e^{i k r}}{r}\right|^{2}}{\left|e^{i k z}\right|^{2}}=|f(\theta)|^{2} .
$$

The flux of the incoming particles is clearly proportional to $\left|e^{i k z}\right|^{2}$, but are the two proportionality constants for the incoming and outgoing fluxes equal? Indeed it can be shown that they are because the momentum of the incomingand the outgoing beams are identical.

The total cross section $\sigma$ is found after integration over the solid angle $d \Omega$, and is given by

$$
\sigma=4 \pi \sum_{l=0}^{\infty}(2 l+1)\left|a_{l}\right|^{2} .
$$

Numerical methods are used to solve the Schrödinger equation in the scattering region and into the intermediate region, where the solution is known analytically. By comparing the two solutions in this region the amplitudes $a_{l}$ can be determined. 


\subsubsection{The phase shift}

The phase shift is a consequence of the scattering process and is due to the potential. To obtain an expression for it we rewrite the plane wave $e^{i k z}$ in Eq. (3.16) by the expansion [32]

$$
e^{i k z}=\sum_{l=0}^{\infty} i^{l}(2 l+1) j_{l}(k r) P_{l}(\cos \theta) .
$$

Without a scattering potential $V(r)$ the wave function in Eq. (3.16) is written

$$
\Psi(r, \theta)=A \sum_{l=0}^{\infty} i^{l}(2 l+1) j_{l}(k r) P_{l}(\cos \theta) .
$$

For large values of $r$ this simplifies to

$$
\Psi(r, \theta)=A \frac{2 l+1}{2 i k r}\left(e^{i k r}-(-1)^{l} e^{-i k r}\right) P_{l}(\cos \theta) .
$$

This is most easily shown by writing $j_{l}(k r)$ as a superposition of the first and second order spherical Hankel functions (see Eqs. (3.14) and (3.15))) and then take the limit $r \gg 1$. The point is that by introducing a potential, the incoming spherical wave is left unchanged (second term in the parentheses above). The first term however is changed, but only the phase is allowed to change. The amplitudes of the incoming and the outgoing spherical waves need to be the same to conserve probability.

Introducing the phase shift $2 \delta_{l}$ in the outgoing spherical wave, Eq. (3.22) can be written

$$
\Psi(r, \theta)=A \frac{2 l+1}{2 i k r}\left(e^{i k r+2 i \delta_{l}}-(-1)^{l} e^{-i k r}\right) P_{l}(\cos \theta) .
$$

This equation is valid in the intermediate region. At this point we go back to Eq. (3.16) and insert the expansion (3.20) in place of $e^{i k z}$ to find

$$
\Psi(r, \theta)=A\left(\frac{2 l+1}{2 i k r}\left[e^{i k r}-(-1)^{l} e^{-i k r}\right]+\frac{2 l+1}{r} a_{l} e^{i k r}\right) P_{l}(\cos \theta) .
$$

Comparing Eqs. (3.23) and (3.24) the coefficients $a_{l}$ are obtained

$$
a_{l}=\frac{1}{2 i k}\left(e^{2 i \delta_{l}}-1\right)=\frac{1}{k} e^{i \delta_{l}} \sin \left(\delta_{l}\right) .
$$


The scattering amplitude $f(\theta)$ and the cross section $\sigma$ can now be expressed in terms of the phase shift

$$
\begin{aligned}
f(\theta) & =\frac{1}{k} \sum_{l=0}^{\infty}(2 l+1) e^{i \delta_{l}} \sin \left(\delta_{l}\right) P_{l}(\cos \theta), \\
\sigma & =\frac{4 \pi}{k^{2}} \sum_{l=0}^{\infty}(2 l+1) \sin ^{2}\left(\delta_{l}\right) .
\end{aligned}
$$

\subsubsection{The low energy limit}

It is possible to show that the phase shift $\delta_{l}$, introduced in Eq. (3.23), can be written [28]

$$
\delta_{l}(k)=c_{l} k^{2 l+1}
$$

in the low energy limit, where $c_{l}$ is an undetermined constant. The coefficients $a_{l}$ can then be expressed as

$$
a_{l}=\frac{1}{2 i k}\left(1+2 i c_{l} k^{2 l+1}-1\right)=c_{l} k^{2 l} .
$$

This confirms the argument we gave previously, and we see that only the $l=0$ partial wave contributes at very low energies. Next we define the scattering length $\alpha$ as the limit of $a_{0}$ when $k \rightarrow 0$,

$$
-\lim _{k \rightarrow 0} a_{0}=-c_{0}=\alpha .
$$

The scattering amplitude $f(\theta)$ can be expressed in terms of $\alpha$ in the low energy limit

$$
f(\theta)=\sum_{l=0}^{\infty}(2 l+1)\left(1+i c_{l} k^{2 l+1}\right) c_{l} k^{2 l} P_{l}(\cos \theta)=c_{0}=-\alpha,
$$

as well as the scattering cross section

$$
\sigma=4 \pi|f(\theta)|^{2}=4 \pi \alpha^{2} .
$$

The total cross section resembles the cross section of a sphere with radius $\alpha$. The extra factor of four must be seen as a quantum mechanical contribution due to the wave nature of the particles. The low energy expressions show that the scattering length is the only quantity of interest at ultracold temperatures. To determine it, the interatomic potential needs to be known. 


\begin{tabular}{|c|c|c|c|}
\hline System & State & Scattering length $\alpha\left(a_{0}\right)$ & Reference \\
\hline \hline${ }^{6} \mathrm{Li}_{2}$ & $X^{1} \Sigma_{g}^{+}$ & $45.5 \pm 2.5$ & {$[33]$} \\
${ }^{6} \mathrm{Li}_{2}$ & $a^{3} \Sigma_{u}^{+}$ & $-2160 \pm 250$ & {$[33]$} \\
${ }^{7} \mathrm{Li}_{2}$ & $X^{1} \Sigma_{g}^{+}$ & $33 \pm 2$ & {$[33]$} \\
${ }^{7} \mathrm{Li}_{2}$ & $a^{3} \Sigma_{u}^{+}$ & -27.3 & {$[34]$} \\
${ }^{6} \mathrm{Li}^{7} \mathrm{Li}$ & $X^{1} \Sigma^{+}$ & $-20 \pm 10$ & {$[33]$} \\
${ }^{6} \mathrm{Li}^{7} \mathrm{Li}$ & $a^{3} \Sigma^{+}$ & $40.9 \pm 0.2$ & {$[33]$} \\
${ }^{23} \mathrm{Na}_{2}$ & $X^{1} \Sigma_{g}^{+}$ & $19.20 \pm 0.30$ & {$[35]$} \\
${ }^{23} \mathrm{Na}_{2}$ & $a^{3} \Sigma_{u}^{+}$ & $62.51 \pm 0.50$ & {$[35]$} \\
${ }^{23}{ }^{8{ }^{85}} \mathrm{Rb}$ & $X^{1} \Sigma^{+}$ & $167_{-30}^{+50}$ & {$[36]$} \\
${ }^{23}{ }^{8{ }^{85}} \mathrm{Rb}$ & $a^{3} \Sigma^{+}$ & $59_{-9}^{+12}$ & {$[36]$} \\
${ }^{39} \mathrm{~K}_{2}$ & $a^{3} \Sigma_{u}^{+}$ & $-51 \pm 0.7$ & {$[37]$} \\
${ }^{87} \mathrm{RbCs}^{3} a^{3} \Sigma^{+}$ & $\left(700_{-300}^{+700}\right)$ or $(176 \pm 2)$ & {$[38]$} \\
$\mathrm{Cs}_{2}$ & $X^{1} \Sigma_{g}^{+}$ & 280.37 & {$[39]$} \\
$\mathrm{Cs}_{2}$ & $a^{3} \Sigma_{u}^{+}$ & 2440 & {$[39]$} \\
\hline
\end{tabular}

Table 3.1: Scattering lengths $\alpha$ for collisions between selected alkali atoms. There may be more recent results available in the literature for some of these systems.

However, the scattering length is extremely sensitive to the interatomic potential, thus, highly accurate Born-Oppenheimer potential curves must be used to compute $\alpha$.

Table 3.1 contains values of $\alpha$ for collisions between selected atomic species. From Table 3.1 two observations are made: 1) The value of $\alpha$ has nothing to do with the radius of the electronic charge cloud (it does not correspond to the size of any physical feature in the atoms), 2) The value of $\alpha$ can be positive, negative, large or small and varies considerably for two rather similar atomic systems.

Perhaps as an aside to the previous discussion we make a comment on the Gross-Pitaevskii (GP) equation. The GP equation provides a mean-field description of the trapped Bose-Einstein condensate wave function $\Phi(\mathbf{r})$

$$
\left(-\frac{1}{2 m} \nabla^{2}(\mathbf{r})+V(\mathbf{r})+\frac{4 \pi \alpha}{m}|\Phi(\mathbf{r})|^{2}\right) \Phi(\mathbf{r})=\epsilon \Phi(\mathbf{r}) .
$$

This is an important equation which we will only comment. The wave function $\Phi(\mathbf{r})$ describes a single atom of mass $m$. The non-linear term, proportional to $|\Phi(\mathbf{r})|^{2}$, represents the mean-field interaction from the other atoms in the condensate on the atom under consideration. The energy of the atom in the presence of the others is denoted $\epsilon$. Notice that the scattering length 
appears in the GP equation and that the atomic interactions are determined by $\alpha$. In fact, the sign and size of $\alpha$ determines whether it is possible to create a Bose-Einstein condensate for a particular atomic species. Only positive values of $\alpha$ (corresponding to repulsive interactions between the atoms in the condensate) allow for stable condensates.

Returning to the scattering problem we plot the wave function $\psi_{0}(r)$, obtained with a very low energy and a realistic interatomic potential $V(r)$. The scattering length can be interpreted as the value of $r$ at the intersection between the asymptote of the wave function and the $r$-axis (see Fig. 3.5).

On a much larger scale the same wave function looks like a sinusoidal wave, as shown in Fig. 3.6. To summarize, Fig. 3.6 shows the wave function on the scale of the de Broglie wavelength, whereas Fig. 3.5 shows the wave function on the scale of the interatomic potential.

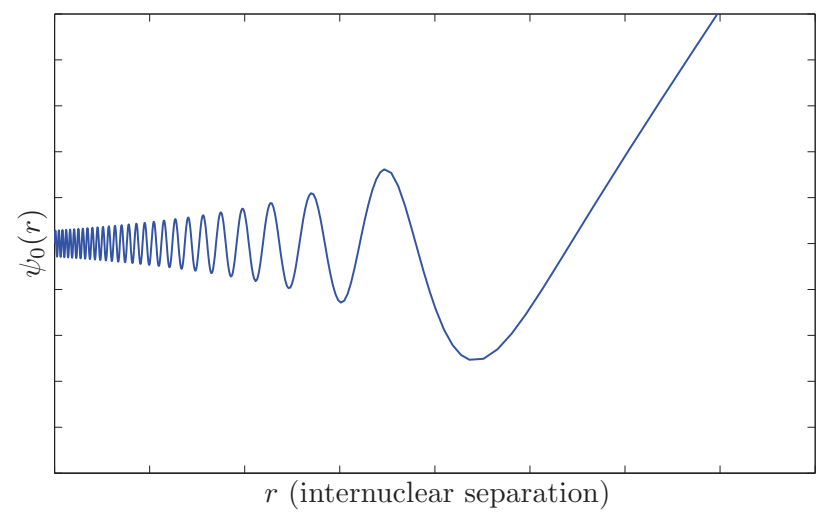

Figure 3.5: The wave function $\psi_{0}(r)$ as a function of the internuclear distance for small and moderate values of $r$. For low energies $(k \rightarrow 0)$ the wave function behaves as indicated in this figure for $r \simeq[0,100] \mathrm{a}_{0}$, depending on the detailed interatomic potential. A straight line may be fitted to the wave function in the region where the rapid oscillations have died out. The scattering length is approximately equal to the value of $r$ where this line intersects the $r$-axis.

\subsubsection{Levinson's theorem}

Levinson's theorem applies to zero energy scattering and is stated below. 


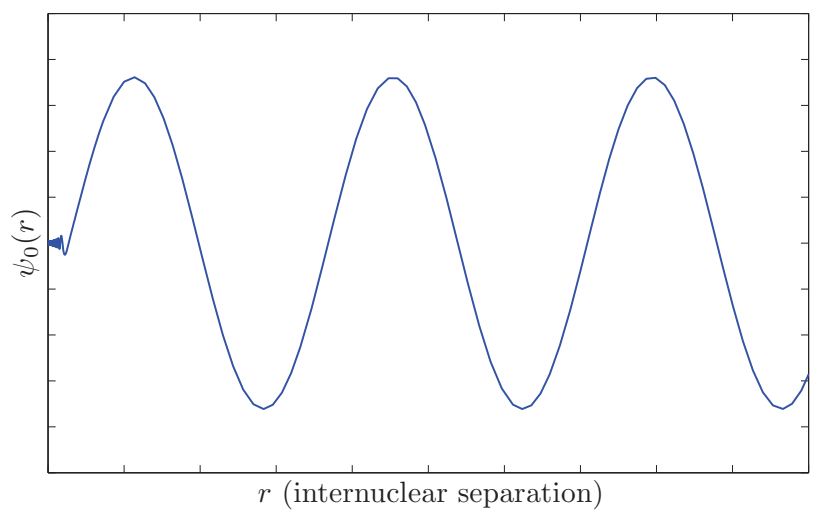

Figure 3.6: The wave function $\psi_{0}(r)$ as a function of the internuclear distance for large values of $r$, typically of the order of $10^{4} a_{0}$. Fig. 3.5 shows a magnification of $\psi_{0}(r)$ for small values of $r$.

Theorem 1 (Levinson's theorem). The phase shift at zero energy $(k \rightarrow 0)$ is related to the number of bound states $N_{l}$ supported by the potential $U_{l}(r)$ as

$$
\delta_{l}=N_{l} \pi
$$

If there exists a zero-energy bound states, Eq. (3.34) needs to be replaced by

$$
\delta_{l}=\left(N_{l}+\frac{1}{2}\right) \pi
$$

It is interesting to study the implication of the theorem on the scattering length $\alpha$. An alternative definition of the scattering length is provided by the expression [28]

$$
\alpha=-\lim _{k \rightarrow 0} \frac{\tan \delta_{0}(k)}{k} .
$$

Consider two slightly different interatomic potential curves $V_{1}(r)$ and $V_{2}(r)$, describing the same system. Assume that $V_{1}$ supports $N_{0}$ bound states, and that $V_{2}$ supports $N_{0}+1$ bound states. The phase shift obtained with $V_{1}$ is $\delta_{0}$. By Levinson's theorem the phase shift obtained from calculations with $V_{2}$ is $\delta_{0}+\pi$. Inserted in Eq. (3.36) a difference of $\pi$ appears quite dramatic 
due to the tangent function, which diverges when the argument approaches $\pi$.

This simplified example illustrates how sensitive the scattering length $\alpha$ may be to small changes in the interatomic potential.

\subsubsection{The optical theorem}

The optical theorem relates the total cross section to the forward scattering amplitude $f(\theta=0)$. The optical theorem is easy to derive. Recall from Eq. (3.26) that the scattering amplitude $f(\theta)$ is

$$
f(\theta)=\frac{1}{k} \sum_{l=0}^{\infty}(2 l+1) e^{i \delta_{l}} \sin \left(\delta_{l}\right) P_{l}(\cos \theta) .
$$

The forward scattering amplitude $f(\theta=0)$ is obtained (note that $P_{l}(1)=1$ ),

$$
f(0)=\frac{1}{k} \sum_{l=0}^{\infty}(2 l+1) e^{i \delta_{l}} \sin \left(\delta_{l}\right) .
$$

The imaginary part of this equation is

$$
\operatorname{Im} f(0)=\frac{1}{k} \sum_{l=0}^{\infty}(2 l+1) \sin ^{2}\left(\delta_{l}\right),
$$

and the right hand side is recognized as the total cross section $\sigma$, multiplied with $k$ and divided by $4 \pi$, cf. Eq. (3.27).

Theorem 2 (The optical theorem).

$$
\sigma=\frac{4 \pi}{k} \operatorname{Im}[f(0)]
$$

\subsection{Inelastic scattering}

Inelastic scattering occurs when energy is exchanged between the internal and the translational degree of freedom. One example is a diatomic molecule that changes rotational level during a collision with an atom. Another example may be found in an atom-atom collisions where the collisions may induce transitions between different hyperfine levels.

Before we discuss inelastic scattering we need to make it clear what we mean by a scattering channel. 
Definition 1. A scattering channel is a quantum state for the complete system before or after the collision has occurred.

We distinguish between closed channels and open channels:

Definition 2. A channel with energy $E_{b}$ is closed if $E_{b}$ is larger than the energy of the system.

Definition 3. A channel with energy $E_{b}$ is open if $E_{b}$ is less or equal to the energy of the system.

In atom-atom collisions, the channels at $t \rightarrow \pm \infty$, when the two atoms are infinitely separated, can be the quantum state $\left|F_{1} M_{F_{1}} F_{2} M_{F_{2}}\right\rangle$ where $F_{1}$ and $F_{2}$ are the quantized total angular momenta of atom one and two respectively. The projections on a space-fixed axis (by convention the $z$-axis) are $M_{F_{i}}$, with $i=1,2$.

Another form of inelastic scattering happens when particles are exchanged between the colliding particles. This is often referred to as reactive scattering and will simply omitted, as it is very unlikely to occur at low energies.

\subsubsection{The multichannel equations}

The goal is to solve the Schrödinger equation and obtain the scattering wave function, the scattering length, the scattering amplitude, the cross section and other quantities of interest. We start by writing the Hamiltonian

$$
H=-\frac{1}{2 \mu} \nabla^{2}(\mathbf{r})+V(\mathbf{r}, \mathbf{R})+H_{\mathrm{int}}(\mathbf{R}) .
$$

The first term is the kinetic energy operator for the relative motion of the collision partners. The interaction potential is denoted $V$ and the Hamiltonian for the internal motion is denoted $H_{\text {int }}$. The eigenfunctions of $H_{\text {int }}$ are assumed to be known together with the eigenvalues,

$$
H_{\text {int }} \psi_{n}(\mathbf{R})=E_{n} \psi_{n}(\mathbf{R}),
$$

where $\mathbf{R}$ denotes the spin and space coordinates necessary to specify the internal motion. This thesis is concerned with two types of collisions, 1) atom-atom collisions and 2) atom-diatomic molecule collisions.

For atom-atom collisions the internal Hamiltonian represents two atoms infinitely separated, and the eigenfunctions $\psi(\mathbf{R})$ are two-atom wave functions $\psi_{F_{1} M_{F_{1}} F_{2} M_{F_{2}}}(\mathbf{R})$, including both the spatial and spin part.

In atom-diatomic molecule collisions we have considered the diatomic molecule as a rigid rotator. The internal Hamiltonian for such a system is then 
the rigid rotator Hamiltonian, and $\psi(\mathbf{R})$ are the corresponding eigenfunctions. The internal state of the atom is assumed to be the ground state, both before and after the collision with the diatomic molecule.

The total wave function is expanded in terms of the eigenfunctions $\psi_{j}(\mathbf{R})$ as

$$
\Psi(\mathbf{r}, \mathbf{R})=\sum_{j} \phi_{j}(\mathbf{r}) \psi_{j}(\mathbf{R})
$$

Inserted in the time-independent Schrödinger equation we get

$$
\sum_{j}\left(-\frac{1}{2 \mu} \nabla^{2}(\mathbf{r})+V(\mathbf{r}, \mathbf{R})+E_{j}-E\right) \phi_{j}(\mathbf{r}) \psi_{j}(\mathbf{R})=0
$$

where Eq. (3.42) has been used. Multiply this equation by $\psi_{i}^{*}(\mathbf{R})$ and integrate over $\mathbf{R}$ to get

$$
\left(-\frac{1}{2 \mu} \nabla^{2}(\mathbf{r})+E_{i}-E\right) \phi_{i}(\mathbf{r})+\sum_{j}\left(\int \psi_{i}^{*}(\mathbf{R}) V(\mathbf{r}, \mathbf{R}) \psi_{j}(\mathbf{R}) d \mathbf{R}\right) \phi_{j}(\mathbf{r})=0 .
$$

The Schrödinger equation alone is usually very challenging to solve. The equation above is even more challenging as it represents a set of coupled Schrödinger equations that need to be solved simultaneously. They are called the close-coupled equations and the solutions are found numerically. The formulation of the theory presented here is termed the diabatic formulation. In this formulation the kinetic energy operator of the relative motion is diagonal in the $\psi_{i}(\mathbf{R})$ basis.

In contrast the adiabatic formulation is often used, in which the expansion (3.43) is replaced by

$$
\Psi(\mathbf{r}, \mathbf{R})=\sum_{j} \phi_{j}(\mathbf{r}) \psi_{j}(\mathbf{r}, \mathbf{R})
$$

where the functions $\psi_{j}(\mathbf{r}, \mathbf{R})$ satisfies

$$
\left[H_{\mathrm{int}}(\mathbf{R})+V(\mathbf{r}, \mathbf{R})\right] \psi_{j}(\mathbf{r}, \mathbf{R})=E_{j}(\mathbf{r}) \psi_{j}(\mathbf{r}, \mathbf{R}) .
$$

With these modifications the derivations of the close-coupled equations proceeds as before. When the separation between the two colliding particles approach infinity, the potential $V$ is zero and the diabatic formulation is again obtained as

$$
E_{j}(\mathbf{r}) \rightarrow E_{j}, \quad \psi_{j}(\mathbf{r}, \mathbf{R}) \rightarrow \psi_{j}(\mathbf{R}) .
$$

We remark that the kinetic energy operator is not diagonal in the basis of the adiabatic basis. Many of these non-diagonal terms can usually be omitted, in particular in the low temperature limit. 


\subsubsection{The $S$-matrix}

In elastic scattering the asymptotic form of the wave function was given by Eq. (3.5). For inelastic scattering a very similar expression can be used. We consider a system with an initial channel $i$, and a final channel $j$, and write the total asymptotic wave function as

$$
\Psi(\mathbf{r}, \mathbf{R})=e^{i k z} \psi_{i}(\mathbf{R})+\sum_{j} \frac{f_{i j}(\theta, \phi)}{r} e^{i k r} \psi_{j}(\mathbf{R}),
$$

in close analogy with Eq. (3.5). The scattering amplitudes $f_{i j}(\theta, \phi)$ describe scattering from channel $i$ to channel $j$ and can be represented as a matrix.

In elastic scattering the phase shift determined the scattering amplitude, the cross section and the scattering length. The scattering matrix holds a similar position in inelastic scattering and is indeed related to the phase shift. The scattering matrix $S$ connects the initial internal state of the system to the final internal state of the system. Let $\psi(-\infty)$ be the initial internal state of the system and $\psi(\infty)$ be the final internal state of the system, we can then define the $S$-matrix as

$$
\psi(\infty)=S \psi(-\infty)
$$

The initial and final internal states can be superpositions of the different wave functions $\psi_{i}(\mathbf{R})$. The $S$-matrix is a function of the total energy and the total angular momentum. The $S$-matrix conserves probability and is therefore unitary

$$
S^{T *} S=I,
$$

where $T$ denotes the transpose of $S$, and $I$ is the identity matrix. The element $S_{i j}$ is the probability amplitude for a transition between the internal states $i$ and $j$, so that the matrix element $\left|S_{i j}\right|^{2}$ gives the corresponding probability. The $S$-matrix is also symmetric, the probability for the reverse transition from $j$ to $i$ must be equal to the transition from $i$ to $j$, due to the principle of time reversal.

To end this section we express the scattering amplitude in terms of the $S$-matrix. Suppose that Eq. (3.45) is further reduced to a set of differential equations in one variable $r$. As for elastic scattering this requires a partial wave expansion. We denote the partial wave quantum number with $l$, as before. The total angular momentum of the colliding particles is $\mathbf{J}$ and the corresponding quantum number is $J$. The scattering amplitude can then be written in the general form

$$
f_{i j}(\theta, \phi)=\frac{1}{2 i\left(k_{i} k_{j}\right)^{1 / 2}} \sum_{j} g_{i j}^{J} Y_{i j}^{J}(\theta, \phi)\left(S_{i j}^{J}-\delta_{i j}\right),
$$


where $g_{i j}^{J}$ are factors that depend on the degeneracy and other constants. The functions $Y_{i j}^{J}(\theta, \phi)$ are the angular momentum eigenfunctions. Only the $S$-matrix depends on the energy. The elastic scattering amplitude can be obtained by using $g_{i j}^{J}=(2 l+1)$ and setting $Y_{j i}^{J}(\theta, \phi)$ equal to $P_{l}(\cos \theta)$. When comparing with Eq. (3.52) the diagonal elements of the $S$-matrix is related to the phase shift as

$$
S=\exp \left(2 i \delta_{l}\right)
$$

Similarly to Eq. (3.52) we may write the elastic and inelastic cross sections in terms of the scattering matrix $S$. These expression must be specialized to the particular collision process studied, and we will not pursue this any further in this chapter, however, we return to these expression in chapters to come. At this point we stress that the scattering matrix is the quantity of interest. To solve the set of coupled equations (3.45) really means to obtain the $S$-matrix.

\subsection{Further reading}

The introduction to cold collision presented in this chapter is based on three books: Griffiths [29] provides a good introduction to one-channel elastic scattering, Murrell and Bosanac [32] give more details and introduce the multichannel equations. As does the book of Child [40], which contains a lot of relevant material, in particular for atom-diatom collisions. The theory of atomic collisions is also treated in detail by Mott and Massey in their book [41]. 


\section{Chapter 4}

\section{Collisions between diatomic molecules and atoms}

In this chapter we will consider atom-molecule collisions in relation to buffergas cooling and matter wave interferometry. The first part of this chapter reviews the buffer-gas cooling process and relates it to the work presented in paper I.

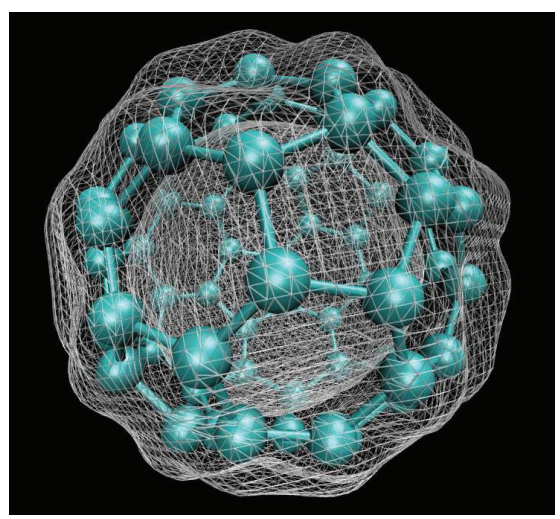

Figure 4.1: $\mathrm{A} \mathrm{C}_{60}$ molecule with the ground state electron density shown (calculated with DFT) [42]. This molecule is among the largest objects whose wavelike properties have been observed in a matter wave interferometer.

The second part of this chapter presents one particular class of matter wave interferometers. We will explain why collisions between atoms and diatomic molecules are relevant also to matter wave interferometers. Finally, 
we comment on the study presented in paper IV.

\subsection{Description of the buffer-gas cooling pro- cess}

Buffer-gas cooling, first proposed by Stwalley [43], stands apart from all the previously discussed cooling methods as it, at least in principle, is completely general. The only requirement is that the particles to be cooled can survive multiple elastic collisions with the buffer-gas.

In chapter 2 we divided the molecular cooling methods into two groups: the direct methods and the indirect methods. Buffer-gas cooling is a direct method. The idea is simple and easy to understand. A cold gas of atoms, usually one of the inert gases e.g. helium, argon or neon, is kept in a container. Hot molecules are loaded into the container and are allowed to collide with the cold inert gas atoms.

After many collisions with the buffer-gas, the molecules should attain a temperature similar to the buffer-gas temperature. The collisions need to be elastic so that only kinetic energy is distributed. Somewhat simplified this is the basic idea of buffer-gas cooling.

This cooling method, as described hitherto, is indeed completely general. It can be applied to cool any species of atoms and molecules (not restricted to diatomic molecules). It does not rely on a cycling transition or subtle spectroscopic properties of the molecule. Yet another advantage is the ability to produce rather large samples of atoms or molecules.

However, we should not disregard the shortcomings of the buffer-gas cooling method. After the cooling process is completed, the particles must be separated from the inert gas of e.g. helium. It is not immediately clear how this can be accomplished.

One method is to introduce a magnetic field in the container (by e.g. an anti-Helmholtz coil configuration) and if the particles (i.e. atoms or molecules) have a sufficiently large magnetic moment $\boldsymbol{\mu}$, they can become trapped: A particle with a magnetic moment $\boldsymbol{\mu}$ experiences an energy shift $\Delta E$ in a magnetic field $\mathbf{B}$, equal to

$$
\Delta E=-\boldsymbol{\mu} \cdot \mathbf{B} .
$$

Particles whose magnetic moments are aligned with the magnetic field, will have lower energies in the regions where $|\mathbf{B}|$ is large. These atoms are called high-field-seekers and tend to gather in regions with a strong magnetic field. Similarly but opposite, particles whose magnetic moments are anti-parallel 
to the magnetic field, tend to gather in regions where the magnetic field strength is low. These are low-field-seekers. By creating a local minimum in the magnetic field the low-field seeking atoms may become trapped in this region, provided that their kinetic energies are insufficient for them to escape (see Fig. (4.2)). Typical magnetic field strengths are of the order $2-4$ Tesla.

Once the cold particles are trapped, the inert gas atoms may be removed. This may be achieved by cooling the walls of the buffer-gas cell to a low temperature, and letting the inert gas condense on the walls.

By relying on this method to remove the inert gas atoms, buffer-gas cooling can no longer be described as a general cooling method. To trap the cold gas of particles they need to have a magnetic moment at least of the order of the Bohr magneton $\mu_{B}$.

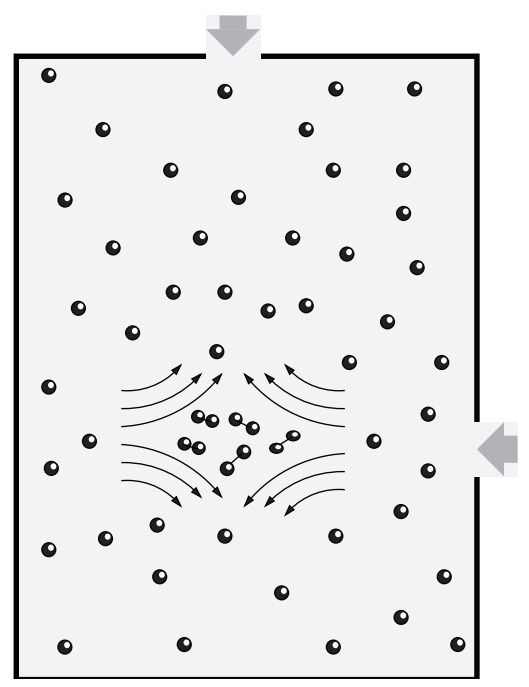

Figure 4.2: The buffer-gas cooling cell. A cold diatomic molecular gas in a low-field-seeking state is trapped by a magnetic field in the middle of the cell. The magnetic field lines are indicated with curved arrows. The inert buffergas atoms, most often helium, are shown as spheres, diatomic molecules as two connected spheres.

The density of the buffer-gas is important. The number of collisions per unit time should be sufficient to cool the particles before they hit the walls of the cell. Otherwise the particles may stick to the wall and be lost. 
After the buffer-gas is removed the diatomic molecules will continue to collide with each other. If the collisions are elastic the sample can be evaporatively cooled as described in chapter 1 . Such molecule-molecule collisions are very complex, even for two diatomic molecules. Few theoretical predictions are available. Generally, the intermolecular potentials need to be known with high accuracy, as the properties of the collisions are very sensitive to the potential in the low energy limit. It is an open question whether potentials with the required accuracy are available for calculations. The electronic, vibrational, rotational, fine and hyperfine structures may provide a very large number of channels, hence, many approximations are necessary to make computations feasible.

Table 4.1 summarizes the capabilities of the buffer-gas cooling method at the present, by listing the diatomic molecular species cooled with this technique along with additional information. In this context we use the word buffer-gas cooling, somewhat misleading, to describe the whole cooling process, including the evaporative cooling.

\begin{tabular}{c|c|c|c}
\hline \hline System & Final temperature $(\mathrm{mK})$ & Density $\left(\mathrm{cm}^{-3}\right)$ & Reference \\
\hline $\mathrm{CaH}$ & $400 \pm 50$ & $4 \times 10^{6}$ & {$[44]$} \\
$\mathrm{NH}$ & 6000 & $10^{12}$ & {$[45]$} \\
$\mathrm{NH}$ & $600-700$ & $10^{8}$ & {$[46]$} \\
$\mathrm{PbO}$ & $4000 \pm 1000$ & $10^{12}$ & {$[47]$} \\
$\mathrm{CrH}$ & 650 & $10^{15}$ & {$[48]$} \\
$\mathrm{MnH}$ & 650 & $10^{15}$ & {$[48]$} \\
\hline \hline
\end{tabular}

Table 4.1: Diatomic molecules cooled with buffer-gas cooling. The temperatures and densities refer to the cold sample of diatomic molecules observed in the experiments.

Finally we mention that many atomic species have been cooled to low temperatures with buffer-gas cooling and subsequent evaporative cooling. Among these are $\mathrm{Eu}, \mathrm{Cr}, \mathrm{Mo}, \mathrm{Dy}, \mathrm{Ho}, \mathrm{Nd}, \mathrm{Pr}, \mathrm{Tb}, \mathrm{Au}, \mathrm{Ag}, \mathrm{Cu}, \mathrm{Mn}, \mathrm{Li}$, $\mathrm{Na}$ and $\mathrm{N}$. Recently the first buffer-gas cooled BEC was also reported for metastable helium $\left({ }^{4} \mathrm{He}^{*}\right)[49]$.

\subsection{Buffer-gas cooling and the present work}

Paper I included in this thesis is relevant for buffer-gas cooling. In this section we describe the relevance of our work, but we do not reproduce the results presented in the paper. Rather we motivate our study. 
As we have seen, buffer-gas cooling in a magnetic field relies on the molecules to be in a trappable state, i.e. the molecules are in a low-field-seeking state. Transitions between trappable and untrappable molecular states can not occur spontaneously. However, collisions between the molecules and the inert gas atoms are not only elastic. Inelastic collisions do occur, in which the internal state of the molecules can change from low-field-seeking states to high-field seeking states. At this stage in the cooling process, where the inert gas atoms are still present in the cell, molecule-molecule collisions do not need to be considered. The number of inert gas atoms are typically five orders of magnitude larger than the number of molecules. Hence, molecule-molecule collisions are extremely rare compared to atom-molecule collisions.

To be more specific, we consider a homonuclear diatomic molecule and an inert gas atom as our system and we are interested in quantities relevant to buffer-gas cooling. This includes among other quantities the cross sections for elastic collisions and for different inelastic collisions. The cross section is defined as [41]

$$
\sigma_{p \rightarrow p^{\prime}}=\frac{\pi}{k_{p}} \sum_{l m_{l} l^{\prime} m_{l}^{\prime}}\left|S_{p l m_{l}}^{p^{\prime} l^{\prime} m_{l}^{\prime}}-\delta_{p p^{\prime}} \delta_{l l^{\prime}} \delta_{m_{l} m_{l}^{\prime}}\right|^{2},
$$

where $p$ is the initial internal state. The partial wave quantum number is $l$ and the projection on a chosen axis is denoted by $m_{l}$. Post collision quantities are marked. The wave vector is denoted $k_{p}$ and $S$ is the $S$-matrix introduced in chapter 3 .

To obtain the $S$-matrix, the potential energy surface describing the interactions between the inert gas atom and the diatomic molecule must be known. This potential is non-central, in contrast to the interatomic potential for two atoms which depends only on $r$. With some approximations the potential energy surface $V$ depends only on two parameters $r$ and $\theta$ (see Fig. $4.3)$.

The approximation that allows us to write $V=V(r, \theta)$ is the rigid rotator approximation. The distance between the two nuclei in the diatomic molecule is fixed to a value we denote by $r_{e}$. The rotation about an axis perpendicular to the molecular axis can be described by the Hamiltonian

$$
H_{\mathrm{rot}}=\frac{\mathbf{p}^{2}}{2 \mu}=\frac{\mathbf{j}^{2}}{2 \mu r_{e}^{2}}=\frac{\mathbf{j}^{2}}{2 I}
$$

where $I$ is the moment of inertia with respect to the rotational axis (perpendicular to the molecular axis), $\mathbf{j}$ is the angular momentum and $\mu$ is the reduced mass of the diatomic molecule. As the separation between the nuclei is constant, the derivative with respect to the internuclear separation, 


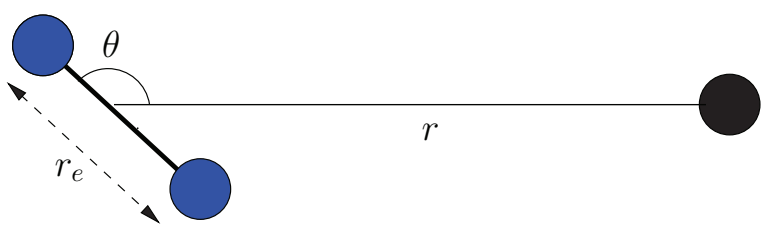

Figure 4.3: Definition of the two parameters $r$ and $\theta$ needed to describe the potential energy surface between a diatomic molecule (blue spheres) and an atom. Notice that the angle $\theta$ indicated in this figure is not the same $\theta$ that was used in the previous chapter to denote the angle of the internuclear axis between the colliding particles.

contained in $\mathbf{p}^{2}$, is zero and the second equality in Eq. (4.3) follows. The Schrödinger equation with this Hamiltonian is solved, and the eigenfunctions are the spherical harmonics $Y_{j m_{j}}(\alpha, \beta)$, where $\alpha$ and $\beta$ describe the orientation of the molecular axis. The corresponding quantized energies are

$$
E_{j}=\frac{j(j+1)}{2 I}
$$

where $j=0,1,2, \ldots$

To obtain the $S$-matrix the close-coupled equations are solved numerically. These equations were presented in chapter three, see Eq. (3.45). Here we will write the set of coupled equations in a different form, obtained by expanding the scattering wave functions $\phi(\mathbf{r}, \mathbf{R})$ in terms of partial wave components $\psi_{l}(r)$ and total angular momentum eigenstates $\mathcal{Y}_{p l}(\mathbf{r}, \mathbf{R})$ :

$$
\phi(\mathbf{r}, \mathbf{R})=\frac{1}{r} \sum_{p l} \psi_{p l}(r) \mathcal{Y}_{p l}(\mathbf{r}, \mathbf{R}) .
$$

The internal state of the system (molecule and atom) is denoted by $p$. The vectors $\mathbf{r}$ and $\mathbf{R}$ were introduced in chapter 3, and denote the relative coordinates between the colliding particles and the internal coordinates respectively. The close-coupled equations are then written (cf. section 3.2)

$$
\left(\frac{d^{2}}{d r^{2}}+k_{p}^{2}-\frac{l(l+1)}{r^{2}}\right) \psi_{p l}(r)=\sum_{p^{\prime} l^{\prime}} 2 \mu\left\langle p l|V(r, \theta)| p^{\prime} l^{\prime}\right\rangle \psi_{p^{\prime} l^{\prime}}(r),
$$

with

$$
k_{p} \equiv 2 \mu\left(E-E_{p}\right)
$$


The wave functions $\psi_{l}(r)$ are obtained numerically. The number of partial waves included in the expansion of the wave function $\phi(\mathbf{r}, \mathbf{R})$ can be large (several hundred), if the temperature is of the order $10 \mathrm{~K}$. Including also the internal states of a diatomic molecule, the number of channels can easily exceed 1000 .

Within the rigid rotator approximation, we investigated the feasibility of buffer-gas cooling of diatomic oxygen, with helium as the buffer-gas. Elastic and inelastic cross sections for collisions between the diatomic oxygen molecules and the helium atoms were computed for energies corresponding to temperatures in the range of $10 \mathrm{~K}$ to $1 \mu \mathrm{K}$. It was discovered that the favorable elastic collisions were more probable than the inelastic collisions by several orders of magnitude, at all temperatures. We refer to paper I for more details and a far more thorough discussion of the calculations and the results.

\subsubsection{Cooling of the rotational degree of freedom}

As the potential between a diatomic molecule and an atom is non-central, the rotational degree of freedom may be cooled, together with the translational degree of freedom. The potential $V(r, \theta)$ couples different states $|q j S l\rangle$, where $j$ is the rotational quantum number, $S$ is the total electronic spin quantum number, $l$ is the partial wave quantum number and $q$ is included to specify any additional quantum numbers. Formally, the possibility of rotational relaxation through collisions with the buffer-gas is due to the matrix elements

$$
\left\langle q j S l|V(r, \theta)| q^{\prime} j^{\prime} S l^{\prime}\right\rangle \neq 0,
$$

and the fact that they are non-zero (cf. Eq. (4.6)). The matrix elements in Eq. (4.8) can be worked out by algebraic techniques, and the detailed expression can be found in paper I. With numerical methods these matrix elements may also be obtained from brute-force integration, but this requires far more computational effort than the solutions written in closed form.

The buffer-gas cooling process for the diatomic oxygen molecule was then simulated on a computer, using the elastic and inelastic cross sections computed. In particular we investigated the effect the collisions had on the initial velocity distribution of the diatomic oxygen molecules, along with the effect on the rotational distribution. To reach equilibrium, we found that the molecules needed $15-20$ collisions with the buffer-gas per molecule considering the translational degree of freedom. However, the rotational degree of freedom required closer to 100 collisions per molecule before equilibrium was reached. To allow for efficient cooling of the rotational degree of freedom the 
potential energy surface $V(r, \theta)$ needs to be far more anisotropic than the $\mathrm{O}_{2}-$ He surface is.

\subsection{Matter wave interferometry and the in- dex of refraction}

The wave-particle duality allows for interferometry with matter. Atoms may behave like waves, with phenomena such as interference and diffraction. The matter waves have a wavelength determined by the de Broglie relation

$$
\lambda_{\mathrm{dB}}=\frac{h}{p},
$$

where $p$ is the momentum of the particle. Although the description of the particle as a wave may appear conceptually challenging, it is nothing new. The wave like properties of electrons and neutrons were investigated long ago, by Davisson and Germer [50] among others. Within this context, the atom interferometer is merely an extension of previous experiments.

We will be concerned with a particular class of atom interferometers, shown schematically in Fig. (4.4).

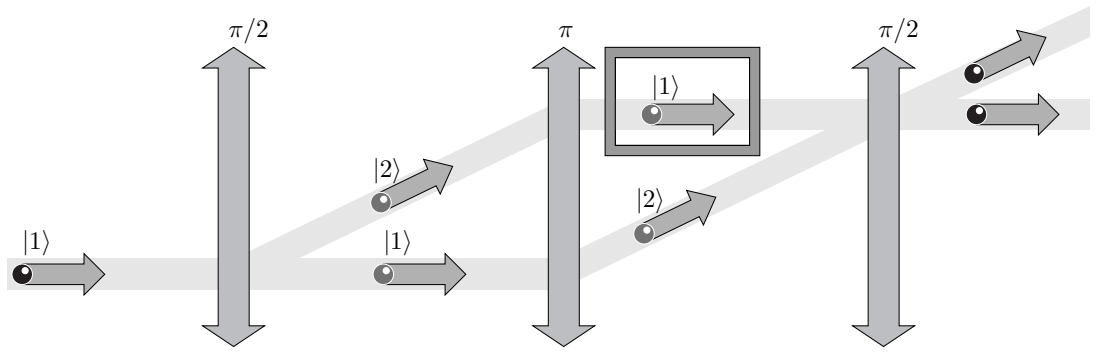

Figure 4.4: An atom interferometer. One beam of particles is split into two beams by a pulse of light (a $\pi / 2$ pulse), then the two beams are deflected and finally recombined by an additional pulse. A box of atoms is introduced in the upper pathway. To the far right an interference pattern can be observed (not drawn). The drawing is a courtesy of Håkon Bjørgen.

The box of particles introduced in the upper pathway induces a phase shift and attenuates the particle beam. This is due to collisions between the particles in the beam and the atoms in the box. The particular effect of these 
collisions on the particle beam, and on the interference pattern, is the topic of the work included as paper IV.

We will further assume that the box is filled with inert gas atoms, and that diatomic molecules are used as the beam. Notice that the collisions occurring within the box are formally identical to the collisions between diatomic molecules and the inert gas atoms in the buffer-gas cooling technique.

Immediately it is not clear how the collisions between the beam particles (hereafter called the projectiles) and the atoms (hereafter called the targets) change macroscopic properties such as the phase shift and the attenuation of the beam. That is, the link between the microscopic collisions and the macroscopic physics, as observed with an interferometer, is not obvious.

The macroscopic wave function, which describes a beam of projectiles along the $x$-axis with a well defined energy $E$, is written

$$
\Psi_{\text {incoming }}(\mathbf{q}, x, t)=\psi(\mathbf{q}) e^{i\left(k_{p} x-E t\right)},
$$

with $k_{p}$ as the wave number and $\psi(\mathbf{q})$ as the wave function for the internal motion of the projectile. The coordinates $\mathbf{q}$ denote the internal coordinates of the projectile.

After traversing a box of length $l$, filled with inert gas atoms, the wave function in Eq. (4.10) is phase shifted and the amplitude is attenuated as $[51]$

$$
\Psi_{\text {trans }}(\mathbf{q}, x, t)=\psi(\mathbf{q}) e^{\left(i k_{p}-E t\right)} e^{i \operatorname{Re}(n) k_{p} l} e^{-\operatorname{Im}(n) k_{p} l},
$$

where "trans" is short for transmitted and $n$ denotes the index of refraction" ${ }^{1}$. The index of refraction is defined as [52]

$$
n=1+2 \pi \rho_{t} \frac{m_{p}+m_{t}}{m_{t}} \frac{\left\langle f\left(k_{r}, \gamma=0\right)\right\rangle}{k_{p}} .
$$

Here $\rho_{t}$ is the density of the target gas, $m_{p}$ is the mass of the projectile particle, $m_{t}$ is the mass of the target particle and $f\left(k_{r}, \gamma=0\right)$ is the forward (i.e. $\gamma=0$ ) scattering amplitude. The index of refraction is a complex quantity because the scattering amplitude $f$ is complex. The wave vector $k_{r}$ is the wave vector for the relative motion, hence

$$
k_{r}=\mu\left|\mathbf{v}_{p}-\mathbf{v}_{t}\right|,
$$

with $\mu$ as the reduced mass (target and projectile), and $\mathbf{v}_{p}$ and $\mathbf{v}_{t}$ as the velocities of the projectile and target, respectively. The brackets in Eq. (4.12)

\footnotetext{
${ }^{1}$ In analogy with the index of refraction defined for light waves in dielectrics, this is the index of refraction for matter waves in a gas of atoms.
} 
mean that the forward scattering amplitude should be averaged over the velocity distribution of the target gas. We assume that the target gas has a well defined temperature.

Notice that the exponential factor involving $\operatorname{Re}(n)$ in Eq. (4.11) contributes with a phase shift $\phi$,

$$
\phi=\operatorname{Re}(n) k_{p} l,
$$

whereas the exponential factor involving $\operatorname{Im}(n)$ attenuates the amplitude of the wave function.

Equation (4.12) is a central part of this theory, as it links the microscopic scattering amplitude to the macroscopic wave function via the refractive index. The forward scattering amplitude can be written [40]

$$
\begin{aligned}
& f\left(k_{r}, \gamma=0\right)= \\
& \frac{1}{2 i} \sum_{J} \sum_{l l^{\prime}} \sqrt{\frac{(2 l+1)\left(2 l^{\prime}+1\right)}{k_{j} k_{j}^{\prime}}} i^{l-l^{\prime}}\left\langle j m_{j} l 0 \mid J M_{J}\right\rangle\left[S_{j l}^{j^{\prime} l^{\prime}}-\delta_{l l} \delta_{j j^{\prime}}\right]\left\langle j^{\prime} m_{j} l^{\prime} 0 \mid J M_{J}\right\rangle,
\end{aligned}
$$

where $\left\langle j m_{j} l 0 \mid J M_{J}\right\rangle$ denotes a Clebsch-Gordon coefficient. The partial wave quantum number is denoted with $l$ and the angular momentum of the diatomic molecule is denoted with $j$. The total angular momentum is

$$
\mathbf{J}=\mathbf{l}+\mathbf{j}
$$

with the corresponding quantum number $J$, and projection $M_{J}$ on the spacefixed $z$-axis. To compute $f\left(k_{r}\right)$, Eq. (4.15) shows clearly that the $S$-matrix needs to be known, hence the solution to the close-coupled equations (4.6) must be obtained.

The scattering amplitude is a function of the energy involved in the collisions, as well as the density $\rho_{t}$ of the target gas. Since the scattering amplitude is averaged over the velocity distribution of the target gas, the temperature of this gas is important. The results we have reported were computed as a function of the projectile velocity and the target gas temperature, and can be found in paper IV.

The results obtained from interferometry experiments may provide information on the validity of the interatomic potential used in the theoretical calculations, when the two are compared. The calculation of interatomic potential surfaces is challenging. The results from matter wave interferometry indicate how well the interatomic potential used in the calculations describes the interactions between the target and the projectile. In this context the 
interferometer should be viewed as an indirect measuring device for the interatomic potential. For good reasons, further explained in paper IV, it is the the ratio

$$
\rho\left(v_{p}\right)=\frac{\operatorname{Re}(n-1)}{\operatorname{Im}(n-1)}
$$

which is measured and compared with theoretical predictions

Most experiments so far have used target gases at room temperature. In paper IV we focus in particular on calculations at low target gas temperatures with low to moderate beam velocities, because at low temperatures the function $\rho\left(v_{p}\right)$ shows clearly both oscillations and resonances (see Fig. 4.5).

In Fig. 4.5 we observe two typical features for the ratio $\rho$. The slow oscillations are called glory oscillations and are related to the number of bound vibrational levels supported by the potential [53]. In Fig. 4.5, only one oscillation is visible (one maximum), but additional maxima may exist at higher projectile velocities.

The rapid oscillations superimposed on the glory oscillations are believed to be resonances due to quasibound levels, which are levels bound by the centrifugal barrier. These resonances are only resolved at low buffer-gas temperatures. For a further discussion, we refer to paper IV.

\subsection{Further reading}

The introduction to the buffer-gas cooling technique was inspired by the PhD thesis "Buffer-Gas Cooling of Diatomic Molecules" by Dimitri Michael Egorov [54]. It contains a lot more information than included here. For more information on matter wave interferometry, see the review "Optics and interferometry with atoms and molecules" by Cronin, Schmiedmayer and Pritchard [55]. 


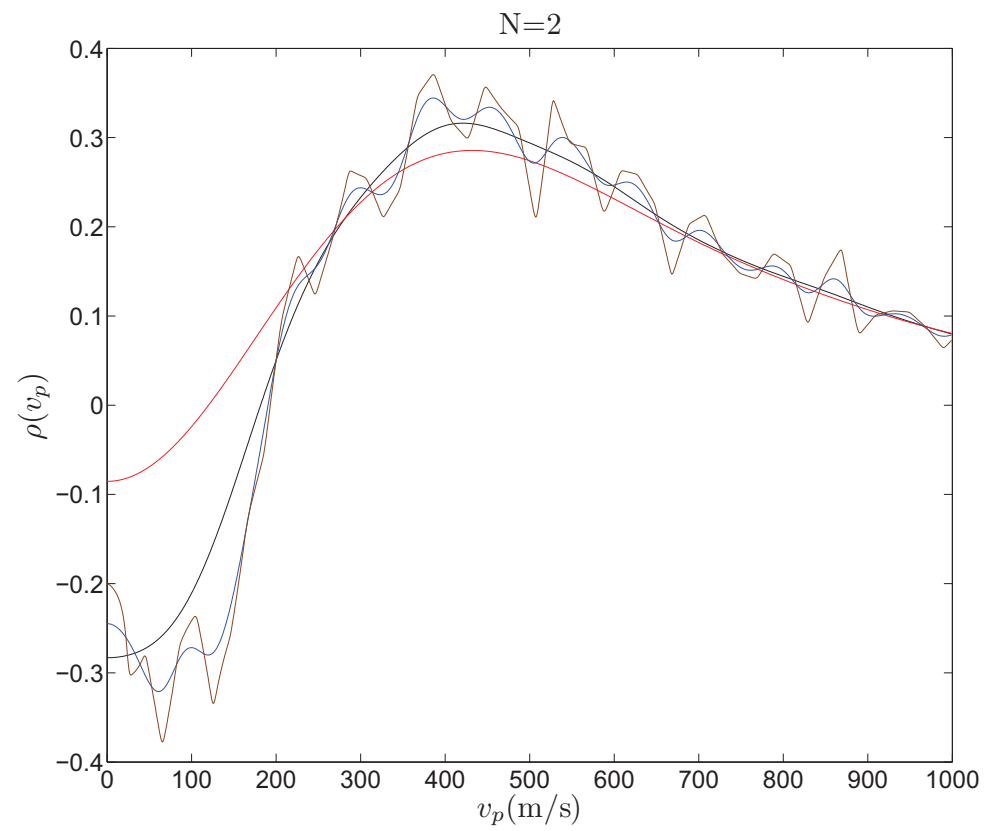

Figure 4.5: Plot of the function $\rho\left(v_{p}\right)$ at four different temperatures, calculated for collisions between diatomic sodium molecules and helium atoms. The sodium molecules act as projectiles, whereas the helium atoms make up the target gas. A color code is used to indicate the temperature of the target gas: red $-4 \mathrm{~K}$, black $-0.1 \mathrm{~K}$, blue $-1 \mathrm{~K}$, brown $-1 \mathrm{mK}$. The rotational level of the $\mathrm{Na}_{2}$ molecules is $N=2$. 


\section{Chapter 5}

\section{Atomic collisions and Feshbach resonances}

In this chapter we introduce the Feshbach resonance using a toy model that encapsulates much of the physics, without introducing unnecessary complexity. We relate the discussion to the Feshbach resonances that appear when two ultracold atoms collide, and motivate parts of our own work presented in papers II and III.

Finally, we present two applications of Feshbach resonances, namely cold diatomic molecule formation and the BCS-BEC crossover, to emphasize the importance of Feshbach resonances in ultracold physics today.

\subsection{Toy model of a Feshbach resonance}

Consider a system with two spin states, $S=1$ and $S=0$. The Hamiltonian can be written (we assume that $\frac{1}{2 m}=1$ )

$$
H=\left(\begin{array}{cc}
-\frac{d^{2}}{d r^{2}}+V_{S}(r) & \Delta \\
\Delta & -\frac{d^{2}}{d r^{2}}+V_{T}(r)+B
\end{array}\right),
$$

with $V_{T}(r)$ as the triplet $(S=1)$ potential and $V_{S}(r)$ as the singlet $(S=0)$ potential. The potentials are defined as (see Fig. 5.1)

$$
V_{S}(r)=\left\{\begin{array}{ll}
-V_{1} & r \leq a \\
0 & r>a
\end{array}, \quad V_{T}(r)=\left\{\begin{array}{ll}
-V_{2} & r \leq a \\
0 & r>a
\end{array} .\right.\right.
$$

The coupling between the different spinstates is represented by $\Delta$. In homonuclear diatomic systems the coupling depends on $r$ and is due to the hyperfine interaction. For Feshbach resonances to occur $\Delta$ must be non-zero. 


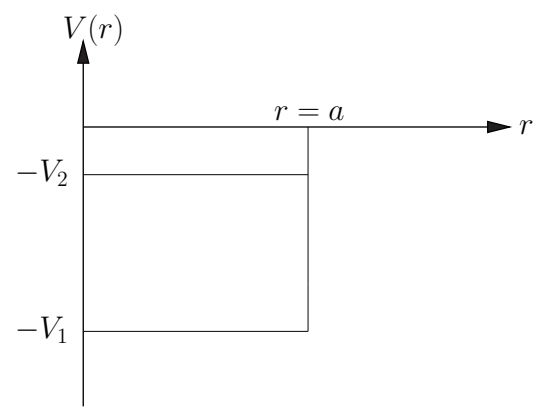

Figure 5.1: The triplet $\left(V_{T}(r)\right)$ and singlet $\left(V_{S}(r)\right)$ potentials.

The time-independent Schrödinger equation reads

$$
\left(\begin{array}{cc}
-\frac{d^{2}}{d r^{2}}+V_{S}(r) & \Delta \\
\Delta & -\frac{d^{2}}{d r^{2}}+V_{T}(r)+B
\end{array}\right)\left(\begin{array}{l}
\psi_{S}(r) \\
\psi_{T}(r)
\end{array}\right)=E\left(\begin{array}{l}
\psi_{S}(r) \\
\psi_{T}(r)
\end{array}\right),
$$

and must be solved for all values of $r$. The states that we consider (scattering states) have energies $E$ larger than zero.

\subsubsection{Long range solution}

At long range $(r \geq a)$ the Hamiltonian simplifies to

$$
H(r \geq a)=-\frac{d^{2}}{d r^{2}}\left(\begin{array}{ll}
1 & 0 \\
0 & 1
\end{array}\right)+\left(\begin{array}{cc}
0 & \Delta \\
\Delta & B
\end{array}\right)
$$

where the last part is non-diagonal. A short piece of algebra yields the eigenvalues as

$$
\lambda_{ \pm}=\frac{1}{2}\left(B \pm \sqrt{B^{2}+4 \Delta^{2}}\right) .
$$

Clearly $\lambda_{+}>0$ while $\lambda_{-}<0$. The non-diagonal part of Eq. (5.4) may be diagonalized with a similarity transformation $Q^{T}\left(\begin{array}{cc}0 & \Delta \\ \Delta\end{array}\right) Q$, with $Q$ defined as

$$
Q=\left(\begin{array}{cc}
2 a \Delta & 2 b \Delta \\
\left(B+\sqrt{B^{2}+4 \Delta^{2}}\right) a & \left(B-\sqrt{B^{2}+4 \Delta^{2}}\right) b
\end{array}\right) .
$$

To save the eyes we have introduced $a$ and $b$ as

$$
a \equiv\left[4 \Delta^{2}+\left(B+\sqrt{B^{2}+4 \Delta^{2}}\right)^{2}\right]^{-1 / 2}, \quad b \equiv\left[4 \Delta^{2}+\left(B-\sqrt{B^{2}+4 \Delta^{2}}\right)^{2}\right]^{-1 / 2},
$$


with the property: $a^{2}+b^{2}=\frac{1}{4 \Delta^{2}}$.

The Schrödinger equation with the diagonalized Hamiltonian is

$$
\left(\begin{array}{cc}
-\frac{d^{2}}{d r^{2}}+\lambda_{+} & 0 \\
0 & -\frac{d^{2}}{d r^{2}}+\lambda_{-}
\end{array}\right)\left(\begin{array}{l}
\psi_{1} \\
\psi_{2}
\end{array}\right)=E\left(\begin{array}{l}
\psi_{1} \\
\psi_{2}
\end{array}\right)
$$

The solutions to this equation can be written

$$
\begin{aligned}
& \psi_{1}(r)=c_{1} \exp \left(-k^{\prime} r\right), \\
& \psi_{2}(r)=\sin (k r)+\tan \left(\delta_{0}\right) \cos (k r),
\end{aligned}
$$

with $k^{\prime}=\sqrt{\lambda_{+}-E}$ and $k=\sqrt{\left|\lambda_{-}\right|+E}$. The phase shift $\delta_{0}$ is not determined, neither is the constant $c_{1}$.

The wave functions $\psi_{1}(r)$ and $\psi_{2}(r)$ are linear combinations of the singlet and triplet wave functions $\psi_{S}(r)$ and $\psi_{T}(r)$. Due to the singlet-triplet interaction, the total spin quantum number is not a good quantum number. This conclusion holds even for real diatomic systems: In highly excited molecular states, in which the internuclear separations is large enough for the singlet and triplet potentials to be identical, the total electronic spin quantum number is not a good quantum number .

\subsubsection{Short range solution}

After having obtained the long-range solution we look for the solution to the short-range Schrödinger equation.

We can rewrite the two coupled differential equations in Eq. (5.3) as four coupled first order equations, by introducing the first derivative of $\psi_{T}$ and $\psi_{S}$ as $\phi_{T}$ and $\phi_{S}$ respectively,

$$
\frac{\mathrm{d}}{\mathrm{d} r}\left(\begin{array}{c}
\psi_{1} \\
\psi_{2} \\
\phi_{1} \\
\phi_{2}
\end{array}\right)=\left(\begin{array}{cc}
\mathbf{0} & \mathbf{1} \\
Q^{T} V Q & \mathbf{0}
\end{array}\right)\left(\begin{array}{l}
\psi_{1} \\
\psi_{2} \\
\phi_{1} \\
\phi_{2}
\end{array}\right)
$$

To simplify the notation we have defined $\mathbf{1} \equiv\left(\begin{array}{ll}1 & 0 \\ 0 & 1\end{array}\right)$ and $\mathbf{0} \equiv\left(\begin{array}{ll}0 & 0 \\ 0 & 0\end{array}\right)$.

In diatomic molecules the coupling between the singlet and triplet spin states is much smaller than both $\left|V_{S}\right|$ and $\left|V_{T}\right|$ at small internuclear separations (corresponding to $r<a$ in the toy model). As $r$ increases, the singlettriplet coupling becomes more important as $\left|V_{S}\right|$ and $\left|V_{T}\right|$ rapidly approach zero. The energy shift due to the interaction with the external magnetic field is much less than both $\left|V_{S}\right|$ and $\left|V_{T}\right|$ (but may be of the same order as $\Delta$ ). 
We adopt these relations among the parameters in our toy model and assume that

$$
\left\{\left|V_{S}\right|,\left|V_{T}\right|\right\} \gg\{|\Delta|,|B|\}, \quad r<a .
$$

It can then be shown that the matrix in Eq. (5.11) has four purely imaginary eigenvalues $\tau=i \alpha, \rho=i \sigma, \tau^{*}=-i \alpha$ and $\rho^{*}=-i \sigma$, with corresponding eigenvectors $\mathbf{v}=\mathbf{u}+i \mathbf{w}, \mathbf{y}=\mathbf{x}+i \mathbf{z}, \mathbf{v}^{*}=\mathbf{u}-i \mathbf{w}$ and $\mathbf{y}^{*}=$ $\mathbf{x}-i \mathbf{z}$. Hence, the solutions to Eq. (5.11) can be written

$$
\begin{array}{r}
\Psi(r)=\left(\begin{array}{c}
\psi_{1}(r) \\
\psi_{2}(r) \\
\phi_{1}(r) \\
\phi_{2}(r)
\end{array}\right)= \\
c_{2}[\sin (\alpha r) \mathbf{u}+\cos (\alpha r) \mathbf{w}]+c_{3}[\cos (\alpha r) \mathbf{u}-\sin (\alpha r) \mathbf{w}]+c_{4}[\sin (\sigma r) \mathbf{x}+\cos (\sigma r) \mathbf{z}] \\
+c_{5}[\cos (\sigma r) \mathbf{x}-\sin (\sigma r) \mathbf{z}] . \quad(5.13)
\end{array}
$$

with $c_{2}, c_{3}, c_{4}$ and $c_{5}$ as unknown constants to be determined from the boundary conditions, and from the continuity requirements on the wave function. The boundary conditions at $r=0$ require that $\psi_{1}(0)=0$ and $\psi_{2}(0)=0$, whereas the continuity requirements can be expressed

$$
\Psi(a)=\left(\begin{array}{c}
c_{1} \exp \left(-k^{\prime} a\right) \\
\sin (k a)+\tan \left(\delta_{0}\right) \cos (k a) \\
-c_{1} k^{\prime} \exp \left(-k^{\prime} a\right) \\
k \cos (k a)-k \tan \left(\delta_{0}\right) \sin (k a)
\end{array}\right) .
$$

The unknown coefficients, including $\tan \delta_{0}$ which is the phase shift, are obtained from the system of equations:

$$
\left(\begin{array}{cccccc}
-\exp \left(-k^{\prime} a\right) & 0 & \mathrm{~s} u_{1}+\mathrm{c} w_{1} & \mathrm{c} u_{1}-\mathrm{s} w_{1} & \mathrm{~s} x_{1}+\mathrm{c} z_{1} & \mathrm{c} x_{1}-\mathrm{s} z_{1} \\
0 & -\mathrm{c} & \mathrm{s} u_{2}+\mathrm{c} w_{2} & \mathrm{c} u_{2}-\mathrm{s} w_{2} & \mathrm{~s} x_{2}+\mathrm{c} z_{2} & \mathrm{c} x_{2}-\mathrm{s} z_{2} \\
k^{\prime} \exp \left(-k^{\prime} a\right) & 0 & \mathrm{~s} u_{3}+\mathrm{c} w_{3} & \mathrm{c} u_{3}-\mathrm{s} w_{3} & \mathrm{~s} x_{3}+\mathrm{c} z_{3} & \mathrm{c} x_{3}-\mathrm{s} z_{3} \\
0 & k \mathrm{~s} & \mathrm{~s} u_{4}+\mathrm{c} w_{4} & \mathrm{c} u_{4}-\mathrm{s} w_{4} & \mathrm{~s} x_{4}+\mathrm{c} z_{4} & \mathrm{c} x_{4}-\mathrm{s} z_{4} \\
0 & 0 & \mathrm{c} w_{1} & \mathrm{c} u_{1} & \mathrm{c} z_{1} & \mathrm{c} x_{1} \\
0 & 0 & \mathrm{c} w_{2} & \mathrm{c} u_{2} & \mathrm{c} z_{2} & \mathrm{c} x_{2}
\end{array}\right)\left(\begin{array}{c}
c_{1} \\
\tan \delta_{0} \\
c_{2} \\
c_{3} \\
c_{4} \\
c_{5}
\end{array}\right)=\left(\begin{array}{c}
0 \\
-\mathrm{s} \\
0 \\
-k \mathrm{c} \\
0 \\
0
\end{array}\right) .
$$

We have introduced the shorthand notation $\mathrm{s}=\sin (k a)$ and $\mathrm{c}=\cos (k a)$ in Eq. (5.15). From the solution of Eq. (5.15) we obtain the scattering length $\alpha$ from the relation

$$
\alpha=-\lim _{k \rightarrow 0} \frac{\tan \delta_{0}}{k} .
$$




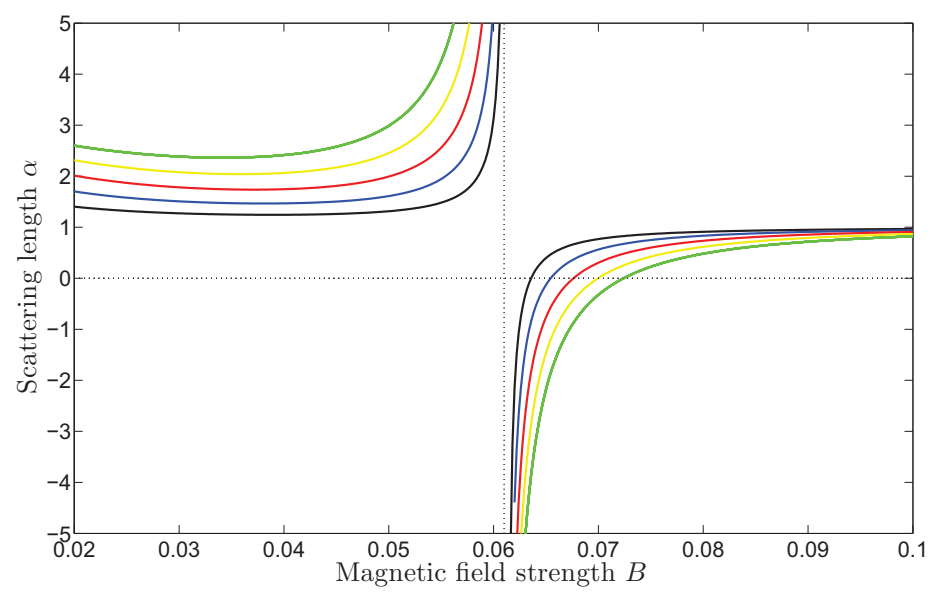

Figure 5.2: Scattering length $\alpha$ vs. external magnetic field strength $B$. The figure shows a Feshbach resonance in the toy model at the magnetic field strength $\simeq 0.06$. The different colors correspond to different coupling strengths $\Delta$ (see Eq. (5.3)). The coupling strengths are $\Delta=0.13$ (green), $\Delta=0.11$ (yellow), $\Delta=0.009$ (red), $\Delta=0.007$ (blue) and $\Delta=0.005$ (black).

\subsubsection{A Feshbach resonance appears}

We choose the potential depths $V_{1}=10$ and $V_{2}=3$, and set the coupling constant $\Delta \simeq 0.01$. For simplicity we assume that $a=1$. The singlet and triplet potentials in zero magnetic field $(B=0)$ are shown in Fig. 5.1. We vary the magnetic field strength, included in Eq. (5.3) with the parameter $B$, between 0 and 0.1. Equation (5.15) is then solved to obtain the phase shift $\delta_{0}$, and the scattering length $\alpha$ follows from Eq. (5.16). The scattering length as a function of magnetic field strength $B$ is shown in Fig. 5.2. The different colors are used to indicate different coupling strengths $\Delta$ between the singlet and triplet states. A strong coupling implies a broad Feshbach resonance and vice verse.

The scattering length is seen to diverge to $\pm \infty$ at a particular magnetic field strength. This is the signature of a Feshbach resonance in the system. By applying a magnetic field the scattering length can be "tuned" to take, in principle, any value between $\pm \infty$. In chapter three we commented that the interactions between the atoms were, at least in part, controlled by the 
scattering length. This implies that a Feshbach resonance enables the experimentalists to control the atomic interactions in an ultracold environment.

An interesting question to answer is this: What happens with the system at the magnetic field strength where the Feshbach resonance appears? To answer this we compute the bound state(s) in the triplet potential. Note that the singlet state does not interact with the magnetic field at all, whereas the triplet state does.

Consider first the situation at $B=0$, shown schematically in Fig. 5.3

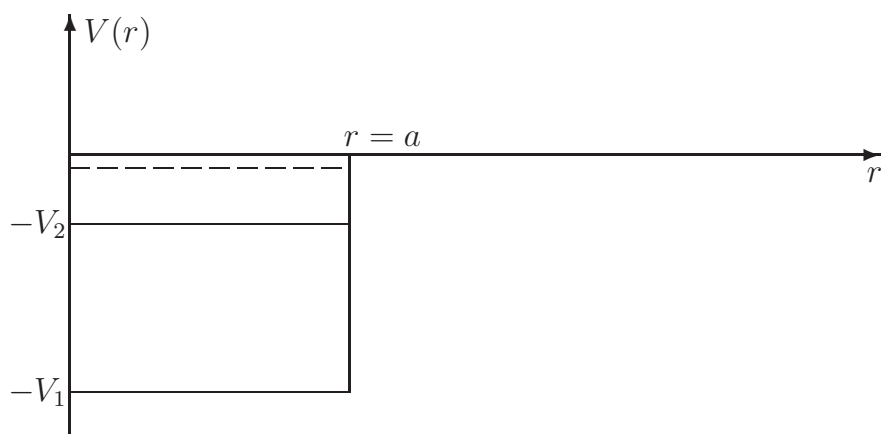

Figure 5.3: The singlet and triplet potentials as well as a bound state in the triplet potential (dashed line). The magnetic field is turned off.

Calculations show that the level in the triplet potential, indicated in Fig. (5.3)) with a dashed line, is bound by approximately 0.062 . When the magnetic field strength is increased, the energies of the bound singlet levels are not affected, whereas the energies of the triplet levels are lifted. It turns out that the triplet potential only supports one energy level. At the magnetic field strength corresponding to the Feshbach resonance, the energy of this level is zero (although bound), as illustrated in Fig. 5.4.

To summarize: The Feshbach resonance occurs when the energy of the bound triplet state corresponds the zero energy asymptote of the singlet potential. In addition, for the Feshbach resonance to appear the singlet and triplet states must be coupled. 


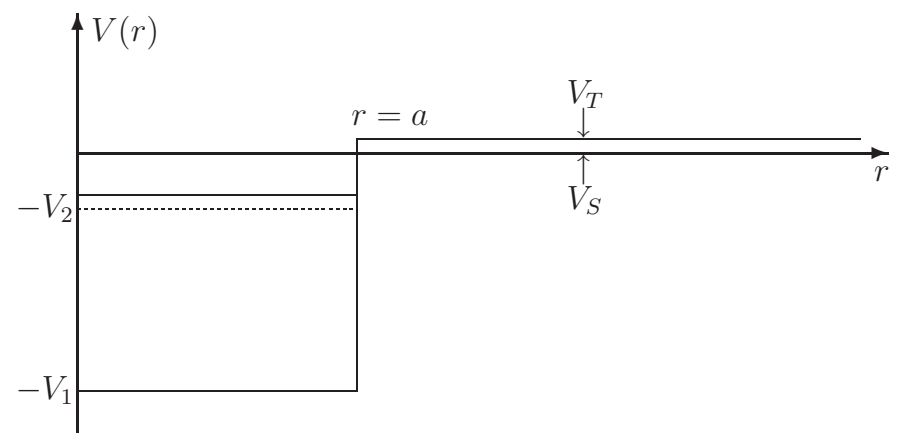

Figure 5.4: A sketch of the effective potentials $\left(V_{S}\right.$ and $\left.V_{T}+B\right)$ at the magnetic field strength where the Feshbach resonance is found. The bound triplet level, indicated with a dashed line in Fig. 5.3, is in resonance with the continuum of states $(E>0)$ for the singlet potential.

\subsection{Feshbach resonances in diatomic alkali mo- lecules}

The system of two ground state alkali atoms of the same species, corresponds to two electronic molecular states, as shown in Fig. 5.5. There is a strong analogy between the occurrences of Feshbach resonances in atomatom collisions and the toy model previously studied. In diatomic systems the Feshbach resonances occur when the energy of a bound molecular state coincides with that of two-colliding atoms.

In this context the diatomic Hamiltonian may be written

$$
H=-\frac{1}{2 \mu} \nabla^{2}+H_{\mathrm{ev}}+H_{\mathrm{hf}}+H_{\mathrm{Z}}
$$

The first term is the kinetic energy of the relative motion of the two nuclei. The second term is the electronic and vibrational contribution, whereas the Hamiltonian $H_{\mathrm{hf}}$ includes the hyperfine interaction. The last term is the Zeeman Hamiltonian, non-zero in a magnetic field.

The hyperfine interaction is very important as it couples the singlet and triplet states in the homonuclear diatomic system. The interaction with external magnetic fields is due to the Zeeman Hamiltonian, which does not interact with the singlet states. However, due to the hyperfine interaction, the singlet and triplet states are coupled and the Zeeman interaction affects all states. 


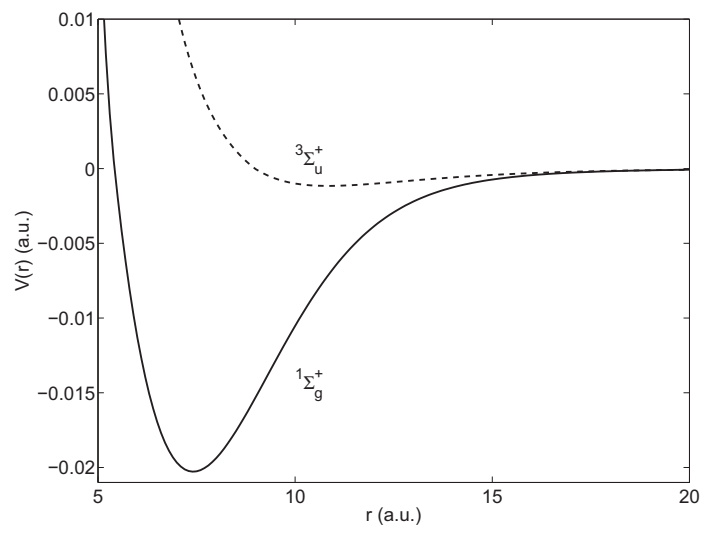

Figure 5.5: The triplet ${ }^{3} \Sigma_{u}^{+}$(dashed line) and singlet ${ }^{1} \Sigma_{g}^{+}$(solid line) potentials corresponding to two ground state atoms.

\subsubsection{Our work}

Papers II and III are relevant to the study of Feshbach resonances. With the Hamiltonian in Eq. (5.17), we solved the close-coupled equations, now written in the form

$$
-\frac{1}{2 \mu} \frac{d^{2}}{d r^{2}} \psi_{a}(r)+\frac{l(l+1)}{2 \mu r^{2}} \psi_{a}(r)+\sum_{b}\left[V_{a b}^{\mathrm{ev}}+V_{a b}^{\mathrm{hf}}+V_{a b}^{Z}\right] \psi_{b}(r)=E \psi_{a}(r) .
$$

The reduced mass is denoted with $\mu$ and

$$
\begin{aligned}
V_{a b}^{\mathrm{ev}} & =\left\langle\phi_{a}(\mathbf{r}, \mathbf{R})\left|H_{\mathrm{ev}}\right| \phi_{b}(\mathbf{r}, \mathbf{R})\right\rangle, \\
V_{a b}^{\mathrm{hf}} & =\left\langle\phi_{a}(\mathbf{r}, \mathbf{R})\left|H_{\mathrm{hf}}\right| \phi_{b}(\mathbf{r}, \mathbf{R})\right\rangle, \\
V_{a b}^{\mathrm{Z}} & =\left\langle\phi_{a}(\mathbf{r}, \mathbf{R})\left|H_{\mathrm{Z}}\right| \phi_{b}(\mathbf{r}, \mathbf{R})\right\rangle .
\end{aligned}
$$

The notation introduced in chapter 3 is used in these equations.

We computed the solutions to the close-coupled equations (5.18), and calculated the magnetic field positions of Feshbach resonances, similar (although far more complicated) to the calculations we did for the toy model.

An immediate question is: How accurate can the magnetic field positions of Feshbach resonances be determined by this kind of ab initio calculations? 
We demonstrate in both papers II and III that the accuracy is very good, provided that the interatomic potentials are highly accurate, including the long-range Van der Waals coefficients.

There are several difficulties involved in solving the close-coupled equations (5.18). Two atoms infinitely well separated are best described in a two-atom basis, with states similar to

$$
|\phi\rangle=\left|q F_{1} M_{F_{1}} F_{2} M_{F_{2}}\right\rangle
$$

where $q$ denotes all quantum numbers besides the total angular momentum quantum numbers $F_{i}$, and their projections $M_{F_{i}}$. As the two atoms approach each other, they interact and behaves as a diatomic molecule when the separation is small. At this point it is very convenient to write the matrix elements in Eqs. (5.19) - (5.21) in terms of molecular basis states, exemplified by the Hund's case (a) states

$$
|\phi\rangle=\left|q \Lambda S \Sigma I \Omega_{I} F M_{F}\right\rangle .
$$

Here $q$ denotes additional quantum numbers, $\Lambda$ is the projection of the electronic orbital angular momentum on the internuclear axis, $S$ is the total electronic spin quantum number and $\Sigma$ is the projection on the internuclear axis, similarly with $I$ and $\Omega_{I}$ for the nuclear spin, and finally, $F$ is the total angular momentum quantum number for the complete system with $M_{F}$ as the projection onto the space-fixed $z$-axis. Relating the two-atom basis states in Eq. (5.22) to the Hund's case basis set in Eq. (5.23) is not trivial. In particular this is due to the Pauli exclusion principle for identical nuclei.

Most studies do not use molecular basis states and completely ignore the molecular hyperfine interaction. One of the questions we wanted to provide an answer to was then: Under what conditions may the molecular hyperfine interaction be neglected, and is it possible to use molecular basis states to considerably simplify the calculations? These questions are addressed in papers II and III included in the present work, and we will not reiterate the arguments here.

\subsection{Applications of Feshbach resonances}

To communicate the importance of the Feshbach resonances in atomic- and molecular physics today we end this thesis with a few examples of how Feshbach resonances are utilized in experiments world wide. 


\subsubsection{Molecular BEC}

In 2003, eight years after the first atomic BEC was observed (see page one of the first chapter), D. Jin et al. [56] wrote in Nature:

...Here we report the direct observation of a molecular BoseEinstein condensate created solely by adjusting the interaction strength in an ultracold Fermi gas of atoms....

At the same time, several other groups observed molecular Bose-Einstein condensates as well $[57,58]$.

Ultracold molecules may be produced by sweeping the magnetic field strength across a Feshbach resonance (see Fig. 5.6), trapping the initial pairs of atoms in highly excited molecular levels. The spatial wave functions of these molecules extend to extremely large internuclear separations.

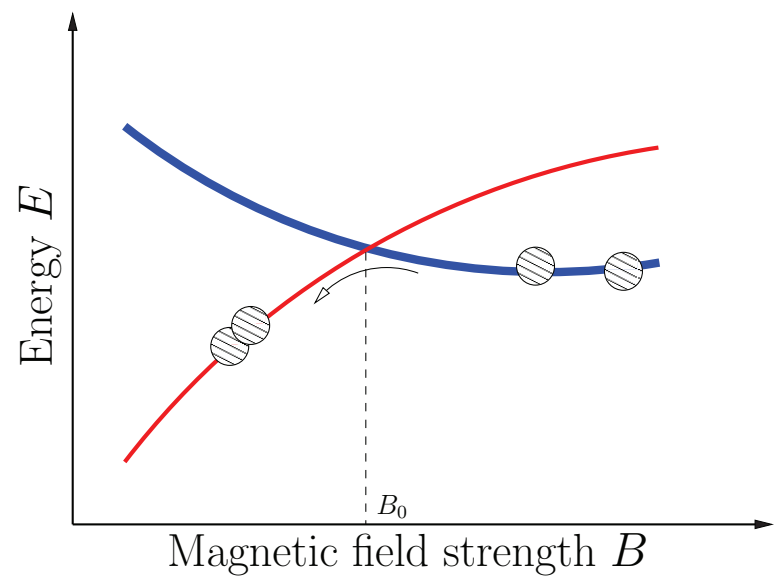

Figure 5.6: Two free atoms are bound in a molecular level of the by a magnetic sweep through a Feshbach resonance at $B=B_{0}$. The red curve shows the energy of a bound molecular state, whereas the blue curve is the energy of two free atoms.

The conversion of atoms to molecules can be very efficient under the right conditions. This depends in particular on the magnetic sweep velocity, i.e $\frac{d B}{d t}$. If the sweep velocity is slow the conversion from atoms to molecules occurs without any increase in temperature. The lifetimes of the highly excited molecular levels depend on the spin statistic. Bosonic species have short 
lifetimes, of the order of milliseconds, whereas fermionic species can have lifetimes of the order of seconds.

The highly excited molecules decay through collisions with free atoms. In bosonic species an atom and a molecule composed of identical atoms are allowed to collide. However, in fermionic species, a fermion may try to collide with a molecule, only to find itself unsuccessful as the fermion is identical to one of the atoms in the molecule. To create diatomic molecules of fermionic atomic species, the two atoms need to be in different states, as $s$-wave collisions between identical fermions are not permitted by the Pauli exclusion principle.

Homonuclear diatomic molecules have been created from Feshbach resonances for the species $\mathrm{Cs}[59], \mathrm{Na}[60],{ }^{87} \mathrm{Rb}[61],{ }^{6} \mathrm{Li}[62],{ }^{40} \mathrm{~K}[63]$, among others. Heteronuclear diatomic molecules have also been created, e.g. ${ }^{6} \mathrm{Li}^{40} \mathrm{~K}[64],{ }^{85} \mathrm{Rb}^{87} \mathrm{Rb}[65]$ and ${ }^{41} \mathrm{~K}^{87} \mathrm{Rb}[66]$.

\subsubsection{BCS-BEC crossover}

The abbreviation BCS stands for Bardeen, Cooper and Schrieffer who proposed the first microscopic theory for superconductivity in 1957 [67]. They later received the Nobel prize in physics for their achievements.

The first definite proof of superfluidity in a system of fermionic atoms came in 2005, when quantized vortices were observed [68]. Somewhat simplified we say that this superfluid is at least approximately described by the BCS theory. The topic of this section is the remarkable possibility of a crossover from a BEC of diatomic molecules to a BCS superfluid of loosely bound atom pairs. We aim to give a short qualitative introduction to the topic.

Several identical fermions can not occupy the same state at the same time, and as a consequence, when $T=0, N$ identical fermions will occupy the $N$ lowest quantum states. By lowest we mean lowest energy. This collection of fermions is sometimes referred to as the Fermi sea, and the energy of the fermions in the most energetic quantum states is called the Fermi energy and is denoted $E_{F}$.

Consider two fermions outside the Fermi sea. Their corresponding wave vectors $\mathbf{k}_{F}$ are equal in magnitude but point in opposite directions. The wave vector $\mathbf{k}_{F}$ is defined by the relation $E_{F}=\frac{\mathbf{k}_{F}^{2}}{2 m}$. This situation was considered by Cooper [69], who found that the energy of the two fermions is less than $2 E_{F}$ for an arbitrary weak attractive interaction between the fermions. This somewhat strange effect is due to the Pauli exclusion principle, and is called Cooper pairing.

However, one should also take into account that the Fermi sea contributes to the Cooper pairing. In fact, Cooper pairing is expected to continue until 
the Fermi sea is sufficiently disturbed so that equilibrium is reached. The BCS theory offers an approximate solution to this challenging many-body problem and describes loosely connected fermion pairs. The different pairs overlap spatially in contrast to a diatomic molecule.

Consider a BEC of diatomic molecules composed of fermions in different spin states. A positive scattering length $\alpha$ is to be associated with a stable condensate. Using a Feshbach resonance the scattering length, and hence the interactions between the fermions, can be adjusted from repulsive to attractive interactions via a region where the scattering length diverges and the interactions are determined by other quantities. At the "attractive side" of the Feshbach resonance, Cooper pairing can occur (see Fig. 5.7). In
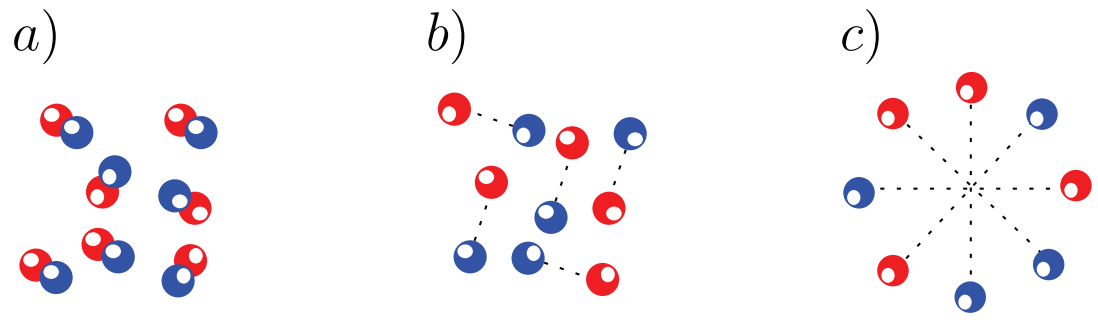

Figure 5.7: Sketch showing the transition from BEC to BCS. a) Pairs of fermions are strongly interacting and has formed composite bosons in a BEC. The scattering length is positive and the interactions are repulsive. b) This is the intermediate region, the "crossover" between a BEC and BCS state. The scattering length $|\alpha|$ is large. The interactions are determined by a different parameter. c) Cooper paring of fermionic atoms, the scattering length is moderate and negative, and the interactions are weak and attractive. Different colors are used to indicate fermions in different spin states.

particular the crossover regime has attracted considerable interest in recent years. The gas is both dilute and strongly interacting. An exact solution to the many-body problem in this regime does not exist at present. Therefore, numerical techniques such as quantum Monte-Carlo simulations are used to investigate the physics in this regime.

\subsection{Further reading}

The review article by Köhler, Góral and Julienne [70] was used in preparing the section on cold molecule formation with Feshbach resonances. The 
presentation of the BCS - BEC crossover is based on the qualitative description given by Cindy Regal in her thesis [71], together with the review article by Giorgini, Pitaevskii and Stringari [72]. Both these references contain vast amounts of information and provide far more advanced treatments. 



\section{Summary}

We provide here a brief summary of the thesis and a few thoughts on how future projects may extend upon the present work.

In this thesis we have seen how the properties of collisions are central both to the atomic and molecular cooling methods, as well as to the dynamics of the cold and ultracold gases. We have seen why elastic collisions are often referred to as good collisions whereas inelastic collisions are referred to as bad collisions. We have encountered cold collisions between different types of particles, i.e. atom-atom collisions, atom-molecule collisions and molecule-molecule collisions. Properties of the latter were not calculated, not because these are not relevant, but because of the complexity involved in such calculations.

Buffer-gas cooling was presented as a completely general cooling method for diatomic molecules, although paramagnetic molecules are preferred as these may be magnetically trapped. In paper I we obtained cross-sections for elastic and inelastic collisions relevant for buffer-gas cooling experiments. Finally we simulated the translational and rotational cooling processes that occur in a buffer-gas cell. There are several possible extensions to this work. For one, the magnetic field should be introduced and its effect on the collisions investigated. Secondly, can we obtain potential energy surfaces of higher accuracy, and what is there to gain by doing this? Finally, how can the vibrational transitions be included?

The Feshbach resonance, introduced in chapter 5, is an important, if not invaluable tool in cold atomic and molecular physics today. Many of its properties were introduced through a toy model with only two channels. However, atom-atom collisions are far more complex, many states need to be included along with an accurate description of the important interactions between them. We have obtained the magnetic field positions of Feshbach resonances in several atomic species (papers II and III), and investigated the effect of the molecular hyperfine structure (paper II) on these. Transition rates for selected processes in atom-atom collisions have been computed (paper III).

We obtained these results by solving the full set of close-coupled equa- 
tions. The construction of the model and the results provided us with valuable insight. To solve the close-coupled equations requires a rather involved numerical calculation. We present two proposals for future extensions of papers II and III

Firstly, is it possible to simplify the calculations by using a small number of channels and an effective Hamiltonian? Such models are available, and it would be interesting to compare the results obtained from them to the results obtained from the full close-coupled model we have used.

Secondly, as we have seen, fermions in identical spin states do not collide through $s$-wave collisions. However, collisions through $p$-waves occur, although suppressed at low temperatures. In many respects the $p$-wave scattering volume replaces the scattering length as the important parameter, describing the properties of such collisions. But to what extent is this analogy meaningful? For instance, how does the scattering volume depend on the position of the bound states in the potential?

In paper IV we consider atom interferometry based on the existence of the de-Broglie matter waves. Collisions between atoms in a gas cell and the atomic or molecular beam in an interferometer are studied. The gas cell is located on one of the paths of the interferometer and collisions between the beam and the gas induces a measurable phase shift. In our calculations we assumed a target gas of cold inert atoms, and computed quantities directly observable in matter-wave interferometry experiments. Compared to experiments, the results may provide valuable information about the interatomic interactions, and the accuracy of the Born-Oppenheimer potentials. Possible extensions to this project would preferably be determined in close collaboration with experimentalists, to make comparison between theory and experiment feasible. 


\section{Bibliography}

[1] Christopher J. Foot. Atomic Physics. Oxford University Press, 2004.

[2] D. J. Wineland and Wayne M. Itano. Laser cooling of atoms. Phys. Rev. A, 20:1521, 1979.

[3] Paul D. Lett, N. Watts, Christoph I. Westbrook, and William D. Phillips. Observation of atoms laser cooled below the doppler limit. Phys. Rev. Lett, 61:169, 1988.

[4] J. Dalibard and C. Cohen-Tannoudji. Laser cooling below the doppler limit by polarization gradients : simple theoretical models. J. Optical Soc. Amer. B, 6:2023, 1989.

[5] Leslie E. Ballentine. Quantum mechanics: A modern development. 1998.

[6] T. Bergman, G. Erez, and H. Metcalf. Magnetostatic trapping fields for neutral atoms. Phys. Rev. A, 35:1535, 1987.

[7] M. H. Andersen, J. R. Ensher, M. R. Matthews, C. E. Wieman, and E. A. Cornell. Observation of bose-einstein condensation in dilute atomic vapor. Science, 269, 1995.

[8] L. D. Landau and E.M. Lifshitz. Quantum Mechanics (Non-relativistic Theory), chapter XVIII, page 601. Elsevier Butterworth-Heinemann, third edition, 1977.

[9] A. E. Leanhardt, T. A. Pasquini, M. Saba, A. Schirotzek, Y. Shin, D. Kielpinski, D. E. Pritchard, and W. Ketterle. Cooling bose-einstein condensates below 500 picokelvin. Science, 301:1513, 2003.

[10] Harold J. Metcalf and Peter van der Straten. Laser Cooling and Trapping. Springer-Verlag (New YorK), 1999.

[11] Max Born and J. Robert Oppenheimer. Zur quantentheorie der molekule. Annalen der Physik, 84:457-484, 1927. 
[12] Per-Olov Löwdin. Quantum theory of many-particle systems. iii. extension of the hartree-fock scheme to include degenerate systems and correlation effects. Phys. Rev., 97(6):1509-1520, Mar 1955.

[13] C. Møller and M.S. Plesset. Note on an approximation treatment for many electron systems. Phys. Rev., 46:618, 1934.

[14] M. W. Schmidt, K. K. Baldridge, J. A. Boatz, S. T. Elbert, M. S. Gordon, J. H. Hensen, S. Koseki, N. Matsunaga, K. A. Nguyen, S. J. $\mathrm{Su}, \mathrm{T}$. L. Wiundus, M. Dupuis, and J. A. Montgomery. General Atomic and Molecular Electronic Structure System. Journal of Comput. Chem., 14:1347-1363, 1993.

[15] Timothy J. Lee and Dylan Jayatilaka. An open-shell restricted hartreefock perturbation theory based on symmetric spin orbitals. Chemical Physics Letters, 201(1-4):1 - 10, 1993.

[16] T. J. Lee, A. P. Rendell, K. G. Dyall, and D. Jayatilaka. Benchmark calculations with correlated molecular wave functions. iv. the classical barrier height of the $\mathrm{h}+\mathrm{h} 2-i \mathrm{~h} 2+\mathrm{h}$ reaction. J. Chem. Phys, 100:74007409, 1994.

[17] Marius Lysebo. Photoassociation of Ultracold Atoms. Master's thesis, University of Oslo, Norway, May 2006.

[18] Markus Greiner, Cindy A. Regal, and Deborah S. Jin. Emergence of a molecular bose-einstein condensate from a fermi gas. Nature, 426:537, 2003.

[19] M. W. Zwierlein, C. A. Stan, C. H. Schunck, S. M. F. Raupach, S. Gupta, Z. Hadzibabic, and W. Ketterle. Observation of bose-einstein condensation of molecules. Phys. Rev. Lett., 91(25):250401, Dec 2003.

[20] T. Bourdel, L. Khaykovich, J. Cubizolles, J. Zhang, F. Chevy, M. Teichmann, L. Tarruell, S. J. J. M. F. Kokkelmans, and C. Salomon. Experimental study of the bec-bcs crossover region in lithium 6. Phys. Rev. Lett., 93(5):050401, Jul 2004.

[21] M. Bartenstein, A. Altmeyer, S. Riedl, S. Jochim, C. Chin, J. Hecker Denschlag, and R. Grimm. Crossover from a molecular bose-einstein condensate to a degenerate fermi gas. Phys. Rev. Lett., 92(12):120401, Mar 2004. 
[22] Cheng Chin, V. V. Flambaum, and M. G. Kozlov. Ultracold molecules: new probes on the variation of fundamental constants. New Journal of Physics, 11:055048, 2009.

[23] D. DeMille. Quantum computation with trapped polar molecules. Phys. Rev. Lett., 88(6):067901, Jan 2002.

[24] O. Dulieu and C. Gabbanini. The formation and interactions of cold and ultracold molecules: new challenges for interdisciplinary physics. Reports on progress in physics, 72:086401, 2009.

[25] Michael S. Elioff, James J. Valentini, and David W. Chandler. Subkelvin cooling no molecules via 'billiard-like' collisions with argon. Science, 302:1940, 2003.

[26] Kevin E. Strecker and David W. Chandler. Kinematic production of isolated millikelvin molecules. Phys. Rev. A, 78(6):063406, Dec 2008.

[27] Leif Veseth. Forelesninger i molekylarfysikk, May 1975.

[28] B. H. Bransden and C. J. Joachain. Physics of Atoms and Molecules. Prentice-Hall, London, 2003.

[29] David Griffiths. Introduction to Quantum Mechanics. 2nd edition edition, 2005.

[30] William H. Press, Saul A. Teukolsky, William T. Vetterling, and Brian P. Flannery. Numerical Recipes 3rd Edition: The Art of Scientific Computing. Cambridge University Press, New York, NY, USA, 2007.

[31] Herbert Goldstein, Charles P. Poole, and John Safko. Classical mechanics, 2002.

[32] J.N. Murrell and S.D. Bosanac. Introduction to the theory of atomic and molecular collisions. 1989.

[33] E. R. I. Abraham, W. I. McAlexander, J. M. Gerton, R. G. Hulet, R. Côté, and A. Dalgarno. Triplet s-wave resonance in li6 collisions and scattering lengths of li6 and li7. Phys. Rev. A, 55(5):R3299-R3302, May 1997.

[34] E. R. I. Abraham, W. I. McAlexander, C. A. Sackett, and Randall G. Hulet. Spectroscopic determination of the $s$-wave scattering length of lithium. Phys. Rev. Lett., 74(8):1315-1318, Feb 1995. 
[35] C. Samuelis, E. Tiesinga, T. Laue, M. Elbs, H. Knöckel, and E. Tiemann. Cold atomic collisions studied by molecular spectroscopy. Phys. Rev. A, 63(1):012710, Dec 2000.

[36] S. B. Weiss, M. Bhattacharya, and N. P. Bigelow. Calculation of the interspecies s-wave scattering length in an ultracold na-rb vapor. Phys. Rev. A, 68(4):042708, Oct 2003.

[37] L. De Sarlo, P. Maioli, G. Barontini, J. Catani, F. Minardi, and M. Inguscio. Collisional properties of sympathetically cooled k39. Phys. Rev. A, 75(2):022715, Feb 2007.

[38] E. Tiesinga, M. Anderlini, and E. Arimondo. Determination of the scattering length of the $a \sigma+3$ potential of rbcs87. Phys. Rev. A, 75(2):022707, Feb 2007.

[39] Cheng Chin, Vladan Vuletić, Andrew J. Kerman, Steven Chu, Eite Tiesinga, Paul J. Leo, and Carl J. Williams. Precision feshbach spectroscopy of ultracold cs2. Phys. Rev. A, 70(3):032701, Sep 2004.

[40] M. S. Child. Molecular Collision Theory. Academic Press (London and New York), 1974.

[41] N.F. Mott and H.S.W. Massey. The theory of atomic collisions. Clarendon Press (Oxford), 1948.

[42] This picture is the own work of the user "Itamblyn" on Wikipedia. To use, you must credit.

See http://en.wikipedia.org/wiki/File:C60_isosurface.png.

[43] W.C. Stwalley. A hybrid laser-magnet trap for spin-polarized atoms. Progress in Quantum Electronics, 8(3-4):203 - 208, 1984.

[44] J. D. Weinstein, R. deCarvalho, T. Guillet, B. Friedrich, and J. M. Doyle. Magnetic trapping of calcium monohydride molecules at millikelvin temperatures. Nature, 395:148, 1998.

[45] D. Egorov, W. C. Campbell, B. Friedrich, S. E. Maxwell, E. Tsikata, L. D. van Buuren, and J. M. Doyle. Buffer-gas cooling of nh via the beam loaded buffer-gas method. The European Physical Journal D Atomic, Molecular, Optical and Plasma Physics, 31:307-311, 2004.

[46] Wesley C. Campbell, Edem Tsikata, Hsin-I Lu, Laurens D. van Buuren, and John M. Doyle. Magnetic trapping and zeeman relaxation of $\mathrm{nh}$ $(x \sigma-3)$. Phys. Rev. Lett., 98(21):213001, May 2007. 
[47] Dima Egorov, Jonathan D. Weinstein, David Patterson, Bretislav Friedrich, and John M. Doyle. Spectroscopy of laser-ablated buffer-gascooled pbo at $4 \mathrm{k}$ and the prospects for measuring the electric dipole moment of the electron. Phys. Rev. A, 63(3):030501, Feb 2001.

[48] Michael Stoll, Joost M. Bakker, Timothy C. Steimle, Gerard Meijer, and Achim Peters. Cryogenic buffer-gas loading and magnetic trapping of crh and mnh molecules. Phys. Rev. A, 78(3):032707, Sep 2008.

[49] S. Charles Doret, Colin B. Connolly, Wolfgang Ketterle, and John M. Doyle. Buffer-gas cooled bose-einstein condensate. Phys. Rev. Lett., 103(10):103005, Sep 2009.

[50] C. Davisson and L. H. Germer. Reflection of electrons by a crystal of nickel. Nature, 119:558, 1927.

[51] J. Schmiedmayer, J. M. Chapman, C. R. Ekstrom, T. D. Hammond, D. Kokorowski, A. Lenef, R. R. Rubenstein, E. Smith, and D. E. Pritchard. Atom Interferometry, page 43. Academic Press, San Diego, 1997.

[52] Caroline Champenois, Marion Jacquey, Steven Lepoutre, Matthias Büchner, Gérard Trénec, and Jacques Vigué. Index of refraction of gases for matter waves: Effect of the motion of the gas particles on the calculation of the index. Phys. Rev. A, 77(1):013621, Jan 2008.

[53] R. B. Bernstein. Semiclassical analysis of the extrema in the velocity dependence of total elastic-scattering cross sections: Relation to the bound states. J. Chem. Phys., 38:2599, 1963.

[54] Dimitri Michael Egorov. Buffer-Gas Cooling of Diatomic Molecules. PhD thesis, Harvard University, Cambridge, Massachusetts, 2004.

[55] Alexander D. Cronin, Jörg Schmiedmayer, and David E. Pritchard. Optics and interferometry with atoms and molecules. Rev. Mod. Phys., 81(3):1051-1129, Jul 2009.

[56] Markus Greiner, Cindy A. Regal, and Deborah S Jin. Emergence of a molecular bose-einstein condensate from a fermi gas. Nature, 426:537, 2003.

[57] S. Jochim, M. Bartenstein, A. Altmeyer, G. Hendl, S. Riedl, C. Chin, J. H. Denschlag, and R. Grimm. unknown. unknown, 302:2101, 2003. 
[58] M. W. Zwierlein, C. A. Stan, C. H. Schunck, S. M. F. Raupach, S. Gupta, Z. Hadzibabic, and W. Ketterle. Observation of bose-einstein condensation of molecules. Phys. Rev. Lett., 91(25):250401, Dec 2003.

[59] Jens Herbig, Tobias Kraemer, Michael Mark, Tino Weber, Cheng Chin, Hanns-Christoph Näger, and Rudolf Grimm. Preparation of a pure molecular quantum gas. Science, 301:1510.

[60] K. Xu, T. Mukaiyama, J. R. Abo-Shaeer, J. K. Chin, D. E. Miller, and W. Ketterle. Formation of quantum-degenerate sodium molecules. Phys. Rev. Lett., 91(21):210402, Nov 2003.

[61] Stephan Dürr, Thomas Volz, Andreas Marte, and Gerhard Rempe. Observation of molecules produced from a bose-einstein condensate. Phys. Rev. Lett., 92(2):020406, Jan 2004.

[62] Kevin E. Strecker, Guthrie B. Partridge, and Randall G. Hulet. Conversion of an atomic fermi gas to a long-lived molecular bose gas. Phys. Rev. Lett., 91(8):080406, Aug 2003.

[63] C. A. Regal, M. Greiner, and D. S. Jin. Observation of resonance condensation of fermionic atom pairs. Phys. Rev. Lett., 92(4):040403, Jan 2004 .

[64] M. Voigt, A.-C.and Taglieber, L. Costa, T. Aoki, W. Wieser, T. W. Hänsch, and K. Dieckmann. Ultracold heteronuclear fermi-fermi molecules. Phys. Rev. Lett., 102(2):020405, Jan 2009.

[65] S. B. Papp and C. E. Wieman. Observation of heteronuclear feshbach molecules from a rb85--rb87 gas. Phys. Rev. Lett., 97(18):180404, Oct 2006.

[66] C. Weber, G. Barontini, J. Catani, G. Thalhammer, M. Inguscio, and F. Minardi. Association of ultracold double-species bosonic molecules. Phys. Rev. A, 78(6):061601, Dec 2008.

[67] J. Bardeen, L. N. Cooper, and J. R. Schrieffer. Microscopic theory of superconductivity. Phys. Rev., 106(1):162-164, Apr 1957.

[68] M. W Zwierlein, J. R Abo-Shaeer, A Schirotzek, C. H Schunck, and W Ketterle. Vortices and superfluidity in a strongly interacting fermi gas. Nature, 435:1047, 2005.

[69] Leon N. Cooper. Bound electron pairs in a degenerate fermi gas. Phys. Rev., 104(4):1189-1190, Nov 1956. 
[70] Thorsten Köhler, Krzysztof Góral, and Paul S. Julienne. Production of cold molecules via magnetically tunable feshbach resonances. Rev. Mod. Phys., 78(4):1311-1361, Dec 2006.

[71] Cindy Regal. Experimental realization of BCS-BEC crossover physics with a Fermi gas of atoms. PhD thesis, University of Colorado, Colorado, 2006 .

[72] Stefano Giorgini, Lev P. Pitaevskii, and Sandro Stringari. Theory of ultracold atomic fermi gases. Rev. Mod. Phys., 80(4):1215-1274, Oct 2008 . 


\section{Part II}

\section{Papers}






\title{
Cold collisions between atoms and diatomic molecules
}

\author{
M. Lysebo* and L. Veseth \\ Department of Physics, University of Oslo, 0316 Oslo, Norway
}

(Received 11 October 2007; published 24 March 2008)

\begin{abstract}
Collision cross sections are calculated for the three systems $\mathrm{O}_{2}-\mathrm{He}, \mathrm{Na}_{2}-\mathrm{Na}$ and $\mathrm{K}_{2}-\mathrm{K}$ at cold and ultracold temperatures. We discuss their relevance for cold molecule formation by buffer-gas cooling and by other cooling methods where collisions between molecules and atoms are important, e.g photoassociation. The molecule is treated as a rigid rotator in the electronic and vibrational ground state. We have calculated ab initio the potential energy surfaces for the three systems with special emphasis on the long range potential which is very important at low energies. With relevance to buffer gas cooling, we have also studied the thermalization process between $\mathrm{O}_{2}$ molecules and $\mathrm{He}$ atoms as determined by the calculated cross sections.
\end{abstract}

DOI: 10.1103/PhysRevA.77.032721

PACS number(s): 34.20.-b, 31.50.Bc, 34.50.- s

\section{INTRODUCTION}

Ultracold molecules are now regularly produced in many laboratories around the world. The cooling of molecules requires some rather sophisticated techniques, due to the fact that molecules cannot be cooled to very low temperatures by the methods applicable to atoms, e.g., laser cooling. Laser cooling is efficient only for two-level systems, whereas molecules are in general multilevel systems [1].

New methods are therefore needed to produce ultracold molecules. One approach that works in particular for the alkalis is to start from samples of ultracold atoms. With samples of ultracold atoms available several procedures have in fact been able to produce even molecular Bose-Einstein condensates, which were first observed in 2003 [2-4].

Feshbach resonances [5] have proved to be a valuable tool in ultracold atomic physics, indeed, also in ultracold molecule formation. In fact the first molecular Bose-Einstein condensates were created from ultracold atoms, converted to molecules by utilizing Feshbach resonances. Unfortunately molecules formed from Feshbach resonances will generally have short lifetimes, and they will normally be formed in highly excited vibrational levels, i.e., be vibrationally very hot.

Different one- or two-color photoassociation $[6,7]$ procedures have also been widely used to create molecules in the ultracold $(<1 \mu \mathrm{K})$ temperature range. Molecules formed by use of photoassociation techniques will also generally end up in high vibrational states, but the end products will in this case depend very much on the atomic species and the detailed photoassociation scheme. In addition, use of the above mentioned methods requires detailed spectroscopic information that may not be available in an accurate manner.

A different and very general cooling method is buffer-gas cooling, first proposed by Stwalley [8]. The method is applicable to atoms as well as molecules. Buffer-gas cooling requires no knowledge of spectroscopic details; the only requirement is of course that reactive collisions with the buffer gas are avoided. Any inert gas may be used as a buffer gas, although ${ }^{3} \mathrm{He}$ is preferred due to its high vapor pressure at

\footnotetext{
*marius.lysebo@fys.uio.no
}

low temperatures. The initially hot molecules disperse their energy onto the buffer-gas atoms and are rethermalized. Unfortunately, buffer-gas cooling does not lead to temperatures that are low enough to enable Bose-Einstein condensates. However, by combining buffer-gas cooling with magnetic trapping [9], molecules may be evaporatively cooled to ultracold temperatures.

For buffer-gas cooling and subsequent evaporative cooling to be effective, it is of paramount importance that the relevant cross sections are favorable. Buffer-gas cooling will be most effective when elastic cross sections are large. Inelastic collisions (state changing collisions) are often referred to as bad collisions in this context. This is not necessarily true, e.g., collisions where the rotational quantum number is reduced will help to cool the rotational degree of freedom. However, inelastic collisions are often associated with trap loss. Collisions that change the Zeeman level from high-field seeking to low-field seeking will certainly cause trap loss for magnetic trapping.

In the present work we have investigated a particular type of cold collisions, namely cold spin-changing collisions between nonpolar diatomic molecules and atoms. Throughout this paper we assume that the molecules are in their ground electronic state and also in their lowest vibrational level $(v=0)$.

Collisions involving molecules represent an extra challenge, as the atom-molecule interaction is certainly more complex than that for atom-atom collisions. In addition, even for cold collisions molecules will have a large variety of available final states (open channels).

Whereas interatomic potentials are often known or can be readily calculated with high accuracy, potential energy surfaces (PES) related to molecular interactions are harder to obtain and might be less accurate. However, the low temperature allows for simplifications. It will, for instance, generally be sufficient to treat the molecule as a rigid rotator with the internuclear separation fixed at the equilibrium distance.

Both atomic- and molecular cross sections are known to be very sensitive to the long-range potential. Often the longrange potential is simply written as $V(r)=-C_{6} / r^{6}$ with $C_{6}$ determined from fitting $V(r)$ to the long range part of the calculated PES. In this study we adopt a different approach and calculate what we believe to be very accurate long-range 


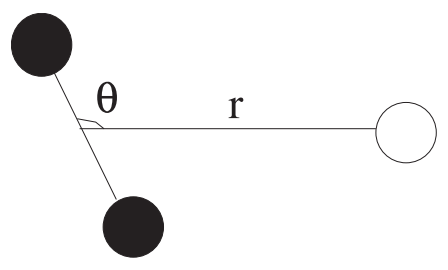

FIG. 1. Definition of $r$ and $\theta$. The diatomic molecule is indicated by the solid circles.

potentials based on the atomic and molecular polarizabilities

Studies similar to the present one on cold atom-diatomic molecule collisions have been presented in several other recent works. Bohn [10] has previously calculated selected cross sections for the $\mathrm{O}_{2}-\mathrm{He}$ interaction with different $\mathrm{O}_{2}$ isotopes [10]. Ultracold collisions between $\mathrm{Na}_{2}\left[{ }^{3} \Sigma_{u}^{+}(v=1)\right]$ and $\mathrm{Na}$ have also been studied [11], as well as collisions involving the same electronic and vibrational states for the systems $\mathrm{Li}_{2}-\mathrm{Li}[12]$ and $\mathrm{K}_{2}-\mathrm{K}$ [13].

\section{SCATTERING CALCULATIONS}

\section{A. Obtaining the $S$ matrix}

Computation of cross sections require solving a large set of coupled differential equations. The method used to solve these equations in the present study has been described elsewhere by several authors $[10,14,15]$. Here we only provide an overview of the method, starting with the Hamiltonian for the whole system (molecule+atom)

$$
H=-\frac{\hbar^{2}}{2 \mu} \nabla^{2}+H_{\mathrm{int}}+V(r, \theta) .
$$

$r$ and $\theta$ are defined according to Fig. 1 and $H_{\text {int }}$ is the internal Hamiltonian for both systems including the molecular fine structure. The total wave function with internal quantum numbers collectively labeled $p$, and orbital angular momentum $L$ with projection $M$ may be written

$$
\Psi_{p L M}=\frac{1}{r} \psi_{p L M}^{\mathcal{J}}(r) \psi_{p}\left(\mathbf{r}_{1}\right) Y_{L M}\left(\theta^{\prime}, \phi^{\prime}\right)
$$

with $\psi_{p}\left(\mathbf{r}_{1}\right)$ as eigenstates of $H_{\text {int }} \cdot \theta^{\prime}, \phi^{\prime}$ is the orientation of a vector from the molecular center of mass to the atom referred to in the laboratory system. The $N$ coupled differential equations are obtained from the Schrödinger equation and may be written [15]

$$
\begin{aligned}
& {\left[\frac{d^{2}}{d r^{2}}+k_{p}^{2}-\frac{L(L+1)}{r^{2}}\right] \psi_{p}^{\mathcal{J}}(r)} \\
& \quad=\sum_{p^{\prime}}\left\langle p L ; \mathcal{J}\left|\frac{2 \mu}{\hbar^{2}} V(r, \theta)\right| p^{\prime} L^{\prime} ; \mathcal{J}\right\rangle \psi_{p^{\prime}}^{\mathcal{J}}
\end{aligned}
$$

In Eq. (3) $p$ and $p^{\prime}$ are internal states for both molecule and atom and $\psi_{p}^{\mathcal{J}}(r)$ is the radial wave function for total angular momentum $\mathcal{J}$. $k_{p}$ is defined as

$$
k_{p}^{2} \equiv \frac{2 \mu}{\hbar^{2}}\left(E-E_{p}\right)
$$

with $\mu$ as the reduced mass and $E_{p}$ as the asymptotic energy in a channel with internal quantum numbers $p$. The number of channels involved in a calculation can be large. All relevant internal molecular states must be included, along with an adequate number of partial waves to ensure convergence for the given energy. Although we include many channels it is important to remember that we do not allow for reactive scattering; thus the molecular bonds cannot be broken during a collision. Reactive scattering should be very limited at most of the temperatures we investigate.

The total angular momentum quantum number $\mathcal{J}$ is defined as

$$
\mathcal{J}=\mathbf{N}+\mathbf{S}+\mathbf{S}+\mathbf{L}
$$

where we have assumed that the atom is without angular momentum ( $s$ state). $\mathbf{N}$ is the molecular angular momentum, $\mathbf{S}$ is the molecular spin, whereas $\mathbf{S}$ is the atomic spin. $\mathbf{L}$ is the partial wave angular momentum representing rotation of molecule + atom about their center of mass. We also introduce $\mathbf{J}_{m}=\mathbf{N}+\mathbf{S}$ as the total molecular angular momentum, and $\mathbf{J}_{1}=\mathbf{s}+\mathbf{L}$.

For a given collision, without a magnetic field, the total angular momentum $\mathcal{J}$ is conserved. However, we do allow $\mathbf{N}$ and $\mathbf{L}$ (and thereby $\mathbf{J}_{m}$ and $\mathbf{J}_{1}$ ) to change in a collision.

To calculate the matrix elements $\left\langle p L ; \mathcal{J}|U| p^{\prime} L^{\prime} ; \mathcal{J}\right\rangle$ the first step is to express the anisotropic potential $V(r, \theta)$ in terms of Legendre polynomials $P_{l}(\cos \theta)$

$$
V(r, \theta)=\sum_{l} v_{l}(r) P_{l}[\cos (\theta)] .
$$

Generally the number of Legendre polynomials included in the sum in Eq. (6) will be large when the potential is strongly anisotropic. With Eq. (6) we may write

$$
\left\langle p L ; \mathcal{J}|U| p^{\prime} L^{\prime} ; \mathcal{J}\right\rangle=\frac{2 \mu}{\hbar^{2}} \sum_{l} v_{l}(r)\left\langle p L ; \mathcal{J}\left|P_{l}(\cos \theta)\right| p^{\prime} L^{\prime} ; \mathcal{J}\right\rangle
$$

Equation (7) may be further simplified by use of the addition theorem for renormalized spherical harmonics

$$
P_{l}(\cos \theta)=\mathbf{C}_{l}(\mathbf{R}) \cdot \mathbf{C}_{l}\left(\mathbf{R}^{\prime}\right)
$$

In Eq. (8) $\mathbf{R}=\left(\theta_{m}, \phi_{m}\right)$ is the direction of the molecular axis referred to the laboratory system. With this definition we have $\theta=\theta_{m}-\theta^{\prime}$.

Next we use the Wigner-Eckart theorem to obtain 


$$
\begin{aligned}
\langle p L ; & \left.\mathcal{J}\left|P_{l}(\cos \theta)\right| p^{\prime} L^{\prime} ; \mathcal{J}\right\rangle \\
& =\left[J_{m}\right]\left[J_{1}\right][N][L]\left[J_{m}^{\prime}\right]\left[J_{1}^{\prime}\right]\left[N^{\prime}\right]\left[L^{\prime}\right] \\
& \times(-1)^{\mathcal{J}-S-s+3 J_{2}+J_{2}^{\prime}+2 J_{1}+2 J_{1}^{\prime}+N+N^{\prime}+L+L^{\prime}}\left\{\begin{array}{ccc}
J_{m} & J_{m}^{\prime} & l \\
J_{1}^{\prime} & J_{1} & \mathcal{J}
\end{array}\right\} \\
& \times\left\{\begin{array}{lll}
N & N^{\prime} & l \\
J_{m}^{\prime} & J_{m} & S
\end{array}\right\}\left\{\begin{array}{lll}
L & L^{\prime} & l \\
J_{1}^{\prime} & J_{1} & s
\end{array}\right\}\left(\begin{array}{ccc}
l & N^{\prime} & N \\
0 & 0 & 0
\end{array}\right)\left(\begin{array}{ccc}
l & L^{\prime} & L \\
0 & 0 & 0
\end{array}\right) .
\end{aligned}
$$

To ease the notation we have defined $\left[J_{m}\right] \equiv \sqrt{\left(2 J_{m}+1\right)}$ and so on. Upon deriving Eq. (9) we have used Eqs. (4.17) and (5.13) of [16]. In Eq. (9) $\{\ldots\}$ denotes $6 j$ symbols and (...) are $3 j$ symbols.

To define the scattering matrix we construct a superposition of the wave functions $\psi_{p}(\mathbf{r})$ which in general defines the electronic, vibrational, and rotational states of the two colliding systems. Before and after a collision the whole system must be represented by the superposition

$$
\Psi\left(\mathbf{r}_{1}\right)=\sum_{p} a_{p} \psi_{p}\left(\mathbf{r}_{1}\right),
$$

where $\left|a_{p}\right|^{2}$ represents the probability of finding the system in eigenstate $\psi_{p}\left(\mathbf{r}_{1}\right)$. Clearly the coefficients $a$ define the state completely for a finite set of quantum numbers $p$. We use the vector $A=\left[a_{p_{1}}, a_{p_{2}}, \ldots, a_{p_{n}}\right]^{T}$ to formally define the scattering matrix by the equation

$$
A_{f}=S A_{i} .
$$

$A_{i}$ and $A_{f}$ are the asymptotic states of the system before and after the collision, respectively. This important scattering matrix ( $S$ matrix) is obtained numerically with the log derivative method first introduced by Johnsen [17]. We have implemented this algorithm and used a local cluster of 30 1.00 GHz Linux PCs to do the actual calculations; this was achieved with a trivial parallelization where each machine gets assigned a specific energy.

\section{B. Calculation of cross sections}

The vast majority of the total CPU time needed for scattering calculations is spent solving the coupled differential equations to obtain the $S$ matrix. Once the $S$ matrix is found, the state to state cross sections can be calculated as a sum over the relevant $S$-matrix elements.

The asymptotic solution $\psi_{p L M}$ outside the range of the potential $V(r, \theta)$ has to satisfy the equation

$$
-\frac{\hbar^{2}}{2 \mu}\left\{\frac{d^{2}}{d r^{2}}-\frac{L(L+1)}{r^{2}}\right\} \psi_{p L M}^{\mathcal{J}}(r)=\left(E-E_{p}\right) \psi_{p L M}^{\mathcal{J}}(r) .
$$

Thus $\psi_{p L M}^{\mathcal{J}}(r)$ can be written

$$
\psi_{p L M}^{\mathcal{J}}(r)=\frac{1}{k_{p}}\left(A_{p L M} e^{-i\left(k_{p} r-L \pi / 2\right)}+B_{p L M} e^{i\left(k_{p} r-L \pi / 2\right)}\right) .
$$

The relevant coefficients $A_{p L M}$ and $B_{p L M}$ are found out of a comparison with the total wave function for a plane incom- ing wave with wave vector $\mathbf{k}_{p}$ in the direction given by $\left(\theta_{k}, \phi_{k}\right)$

$$
\begin{aligned}
e^{i \mathbf{k}_{p} \cdot \mathbf{R}^{\prime}} \psi_{p}= & \frac{2 \pi}{k_{p} r} \sum_{L M}\left\{i^{L} Y_{L M}^{*}\left(\theta_{k}, \phi_{k}\right)\right. \\
& \left.\times\left(e^{i\left(k_{p} r-L \pi / 2\right.}-e^{-i\left(k_{p} r-L \pi / 2\right)}\right) Y_{L M}\left(\theta^{\prime}, \phi^{\prime}\right)\right\} \psi_{p} .
\end{aligned}
$$

It is found that $A_{p L M}=-B_{p L M}=-\left(2 \pi / i k_{p}\right) i^{L} Y_{L M}^{*}\left(\theta_{k}, \phi_{k}\right)$. From the definition of the $S$-matrix elements we may write $B_{p^{\prime} L^{\prime} M^{\prime}}=S_{p L M}^{p^{\prime} L^{\prime} M^{\prime}} A_{p L M}$. We use this to construct a total wave function with an incoming plane wave in internal state $p$ and an outgoing spherical wave in a superposition of different internal states $p^{\prime}$

$$
\begin{aligned}
\Psi_{p}= & \frac{2 \pi}{i r k_{p}^{1 / 2}}\left(\sum_{L M} i^{L} Y_{L M}^{*}\left(\theta_{k}, \phi_{k}\right) k_{p}^{-1 / 2} e^{-i\left(k_{p} r-L \pi / 2\right)} Y_{L M}\left(\theta^{\prime}, \phi^{\prime}\right) \psi_{p}\right. \\
& +\sum_{p^{\prime} L^{\prime} M^{\prime}} i^{L^{\prime}} Y_{L M}^{*}\left(\theta_{k}, \phi_{k}\right) k_{p^{\prime}}^{-1 / 2} S_{p L M}^{p^{\prime} L^{\prime} M^{\prime}} \\
& \left.\times e^{i\left(k_{p^{\prime}} r-L^{\prime} \pi / 2\right)} Y_{L^{\prime} M^{\prime}}\left(\theta^{\prime}, \phi^{\prime}\right) \psi_{p^{\prime}}\right) .
\end{aligned}
$$

By comparison with the well known total wave function for large $r$

$$
\Psi_{p}=\psi_{p} e^{i \mathbf{k}_{p} \cdot \mathbf{r}}+\sum_{p^{\prime}} f_{p^{\prime}} \frac{e^{i k_{p^{\prime}} r}}{r} \psi_{p^{\prime}}
$$

it is possible to identify the scattering amplitude $f_{p^{\prime}}\left(\theta^{\prime}, \phi^{\prime}\right)$ for scattering in direction $\left(\theta^{\prime}, \phi^{\prime}\right)$,

$$
\begin{aligned}
f_{p^{\prime}}\left(\theta^{\prime}, \phi^{\prime}\right)= & \frac{2 \pi}{i\left(k_{p} k_{p^{\prime}}\right)^{1 / 2}} \sum_{L M} i^{L} Y_{L M}^{*}\left(\theta_{k}, \phi_{k}\right) \sum_{L^{\prime} M^{\prime}} e^{-i L \pi / 2} \\
& \times\left[S_{p L M}^{p^{\prime} L^{\prime} M^{\prime}}-\delta_{p p^{\prime}} \delta_{L L^{\prime}} \delta_{M M^{\prime}}\right] Y_{L^{\prime} M^{\prime}}\left(\theta^{\prime}, \phi^{\prime}\right) .
\end{aligned}
$$

Closely related to the scattering amplitude is the differential cross section, which may be calculated from the connection $d \sigma / d \Omega=\left(k_{p^{\prime}} / k_{p}\right)\left|f_{p^{\prime}}\right|^{2}$. In addition, we average over the incident directions $\left(\theta_{k}, \phi_{k}\right)$ to find that the cross section is given by the equation

$$
\sigma_{p \rightarrow p^{\prime}}=\frac{\pi}{k_{p}^{2}} \sum_{L M L^{\prime} M^{\prime}}\left|S_{p L M}^{p^{\prime} L^{\prime} M^{\prime}}-\delta_{p p^{\prime}} \delta_{L L^{\prime}} \delta_{M M^{\prime}}\right|^{2} .
$$

\section{POTENTIAL ENERGY SURFACES}

\section{A. Short range potential}

We have investigated the three systems $\mathrm{O}_{2}-\mathrm{He}, \mathrm{Na}_{2}-\mathrm{Na}$, and $\mathrm{K}_{2}-\mathrm{K}$. For all systems we have needed to calculate potential energy surfaces (PES). For small internuclear separations $(r<20$ a.u.) we have used the quantum chemistry package GAMESS [18]. In these calculations we started with a UHF self-consistent field molecular wave functions for all the systems. In particular, for the $\mathrm{O}_{2}-\mathrm{He}$ complex we need 
TABLE I. Basis sets used with GAMESS to calculate the different short range potential energy surfaces. Similar types of Dunning's correlation consistent basis sets are used for $\mathrm{O}_{2}-\mathrm{He}$ and $\mathrm{Na}_{2}-\mathrm{Na}$, whereas the TZV (triple valence) basis set is used for $\mathrm{K}_{2}-\mathrm{K}$.

\begin{tabular}{cccc}
\hline \hline System & $\begin{array}{c}\text { Basis set used for atoms } \\
\text { in the molecule }\end{array}$ & $\begin{array}{c}\text { Basis set for } \\
\text { the lone atom }\end{array}$ & $\begin{array}{c}\text { Basis } \\
\text { ref. }\end{array}$ \\
\hline $\mathrm{O}_{2}-\mathrm{He}$ & aug-cc-pCVTZ & aug-cc-pCVTZ & {$[21-23]$} \\
$\mathrm{Na}_{2}-\mathrm{Na}$ & cc-pVTZ & cc-pVTZ & {$[23]$} \\
$\mathrm{Ka}_{2}-\mathrm{Ka}$ & $\mathrm{TZV}$ & $\mathrm{TZV}$ & {$[24]$} \\
\hline \hline
\end{tabular}

the UHF wave function due to the Hartee-Fock instability of $\mathrm{O}_{2}$ as reported previously by van Lenthe and van Duijneveldt [19]. The Hartree-Fock wave function is used as a starting point for a more accurate treatment based on the MøllerPlesset perturbation theory.

We have calculated second order energy corrections with MP2 [20]. The basis sets used are reported in Table I [21-24].

No frozen core orbitals have been kept in our MP2 calculations. To obtain the internuclear interactions we have used the supermolecular approach [25]. This implies that for all geometries three calculations are needed. First we calculate the MP2 energy for the total system consisting of atom + molecule; thereafter, we perform calculations with the molecule together with a ghost atom.

A ghost atom does not have a positively charged nucleus but the atomic orbitals are kept. Here we use the counterpoise correction method [26] with ghost atoms to reduce the basis set superposition error. For each geometry this involves also a calculation of the MP2 energy with the atom and the ghost molecule. Finally, to obtain the PES, the energies of the two calculations involving ghost basis sets are added and the result is subtracted from the energy of the whole complex. Generally for two atoms or molecules $A$ and $B$ at geometry $\mathbf{G}$ we calculate

$$
V_{A B}(\mathbf{G})=E_{A B}(\mathbf{G})-E_{A}(\mathbf{G})-E_{B}(\mathbf{G}),
$$

with $E_{A B}$ as the energy obtained for both systems $A$ and $B$, whereas $E_{A}$ and $E_{B}$ are the respective energies obtained with only system $A$ or system $B$. For all calculations the basis sets are the same as for the full calculation $A B$.

\section{B. Long range potentials}

At long distances the interaction potential between an atom and a nonpolar diatomic molecule takes the approximate form (cf. Fig. 1):

$$
\begin{aligned}
V(r, \theta)= & -\frac{1}{r^{6}}\left[C_{6}^{(0)}+C_{6}^{(2)} P_{2}(\cos \theta)\right] \\
& -\frac{1}{r^{8}}\left[C_{8}^{(0)}+C_{8}^{(2)} P_{2}(\cos \theta)+C_{8}^{(4)} P_{4}(\cos \theta)\right] .
\end{aligned}
$$

The dispersion coefficients (Van der Waals coefficients) $C_{6}^{(0)}$,
$C_{6}^{(2)}, C_{8}^{(0)}, C_{8}^{(2)}$, and $C_{8}^{(4)}$ may be expressed in terms of the polarizabilities of the atom and the molecule. The lowestorder coefficients $C_{6}^{(0)}$ and $C_{6}^{(2)}$ are determined by the dipole polarizabilities, whereas quadrupole and octupole polarizabilities are needed to determine the coefficients $C_{8}^{(0)}, C_{8}^{(2)}$, and $C_{8}^{(4)}$.

The frequency dependent (dynamic) dipole polarizability for an atom is given by the expression

$$
\alpha_{1}^{A}(\omega)=-\sum_{k(\neq n)} \frac{\left|\left\langle\psi_{k}\left|\sum_{i=1}^{N} z_{i}\right| \psi_{n}\right\rangle\right|^{2}}{E_{n}-E_{k} \pm \omega},
$$

where $E_{k}$ and $\left|\psi_{k}\right\rangle$ respectively denote eigenvalues and eigenstates of the atomic system with $N$ electrons. The double sign in the denominator indicates a sum of two terms, one with + and the other with -. For a diatomic molecule one has to consider the polarizability $\alpha_{\|}(\omega)=\alpha_{z z}$ along the molecular axis and the polarizability $\alpha_{\perp}(\omega)=\alpha_{x x}(\omega)=\alpha_{y y}(\omega)$ perpendicular to the axis. The expression for $\alpha_{\|}(\omega)$ is identical to that of Eq. (21), whereas $\alpha_{\perp}(\omega)$ is obtained from Eq. (21) by replacing $z_{i}$ with $x_{i}$ or $y_{i}$. Finally, the polarizabilities that will be needed for a diatomic molecule are the average polarizability

$$
\bar{\alpha}(\omega)=\frac{1}{3}\left[2 \alpha_{\perp}(\omega)+\alpha_{\|}(\omega)\right]
$$

and the anisotropy

$$
\Delta \alpha(\omega)=\alpha_{\|}(\omega)-\alpha_{\perp}(\omega) .
$$

Unfortunately, Eq. (21) is not very useful to compute dynamic polarizabilities, as singular frequencies will be encountered at the excited energies $E_{k}$. However, it turns out that the dispersion coefficients are actually determined by the polarizabilities at imaginary frequencies $i \eta$. At imaginary frequencies there are no singularities and the polarizabilities are real positive numbers that decrease monotonically from their maximum at $\eta=0$. Similar properties are also found for the quadrupole and octupole polarizabilities at imaginary frequencies.

A method for accurate computation of dynamic polarizabilities at imaginary frequencies has been presented in a previous work by one of the present authors [27]. Diagrammatic many-body theory is used, with complete inclusion of all diagrams representing up to two interactions with the electronic repulsion term $1 / r_{12}$. This means a substantial inclusion of electron correlation and, with reference to Eq. (21), that the polarizabilities are expected to be computed with very accurate eigenvalues and eigenstates. The same technique also applies to the quadrupole and octupole polarizabilities; the dipole components merely have to be replaced by the relevant quadrupole or octupole moments.

The expressions for the dispersion coefficients $C_{6}^{(0)}$ and $C_{6}^{(2)}$ are fairly simple [28,29]:

$$
C_{6}^{(0)}=\frac{3 \hbar}{\pi} \int_{0}^{\infty} \alpha_{1}^{A}(i \eta) \bar{\alpha}(i \eta) d \eta,
$$


TABLE II. Computed dipole polarizabilities (a.u.) at the imaginary frequencies $i \eta\left(\right.$ a.u. ) for the electronic ground states of $\mathrm{O}_{2}$ and $\mathrm{Na}_{2}$. The computed values are at the respective equilibrium internuclear distances.

\begin{tabular}{|c|c|c|c|c|}
\hline \multirow[b]{2}{*}{$\eta$} & \multicolumn{2}{|c|}{$\mathrm{O}_{2}$} & \multicolumn{2}{|c|}{$\mathrm{Na}_{2}$} \\
\hline & $\alpha_{\|}$ & $\alpha_{\perp}$ & $\alpha_{\|}$ & $\alpha_{\perp}$ \\
\hline 0.00 & 14.68 & 5.73 & 347.2 & 184.3 \\
\hline 0.10 & 13.84 & 5.60 & 164.0 & 109.5 \\
\hline 0.20 & 12.28 & 5.28 & 45.5 & 42.3 \\
\hline 0.30 & 10.63 & 4.88 & 15.9 & 18.5 \\
\hline 0.40 & 9.09 & 4.45 & 7.17 & 9.76 \\
\hline 0.50 & 7.74 & 4.03 & 3.81 & 5.95 \\
\hline
\end{tabular}

$$
C_{6}^{(2)}=\frac{\hbar}{\pi} \int_{0}^{\infty} \alpha_{1}^{A}(i \eta) \Delta \alpha(i \eta) d \eta .
$$

The expressions for the higher-order dispersion coefficients $C_{8}^{(0)}, C_{8}^{(2)}$, and $C_{8}^{(4)}$ are rather complex and are not reproduced here. Complete expressions can be found in the papers by Bishop and Pipin [28] and Hohm [29], and references therein. Table II gives computed dipole polarizabilities at some selected imaginary frequencies for the diatomic molecules $\mathrm{O}_{2}$ and $\mathrm{Na}_{2}$. The computed dispersion coefficients are shown in Table III. The results presented are for the systems $\mathrm{He}-\mathrm{O}_{2}, \mathrm{Na}-\mathrm{Na}_{2}$, and $\mathrm{K}-\mathrm{K}_{2}$, which are the systems of current interest.

\section{IV. $\mathrm{O}_{2}$-He SYSTEM}

In the present work we consider the ${ }^{3} \mathrm{He}$ isotope and calculate cross sections for collisions with ${ }^{16} \mathrm{O}_{2}$ molecules. The $\mathrm{O}_{2}$ molecule is treated as a rigid rotator with an internuclear separation of $r_{e}=1.2165 \AA$. We discuss how the calculated cross sections are relevant for both buffer-gas cooling of $\mathrm{O}_{2}$ molecules as well as for magnetic trapping of $\mathrm{O}_{2}$ molecules with $\mathrm{He}$ atoms present.

The ground state of the $\mathrm{O}_{2}$ molecule has the designation ${ }^{3} \Sigma_{g}^{-}$. The rotational structure together with the $\mathbf{J}=\mathbf{N}+\mathbf{S}$ fine structure are shown in Fig. 2 for the ground electronic and vibrational state [30]. Note that even rotational levels are not allowed in the ground state due to the Pauli principle which requires the total molecular wave function to be symmetric whenever the nuclear spin is equal to zero [31].

The calculated, short range PES is reported in Table IV. From Table IV and the PES it is readily seen that the He atom is most strongly repelled from the $\mathrm{O}_{2}$ molecule when (20)].

TABLE III. Computed dispersion coefficients (a.u.) [cf. Eq.

\begin{tabular}{cccccc}
\hline \hline & $C_{6}^{(0)}$ & $C_{6}^{(2)}$ & $C_{8}^{(0)}$ & $C_{8}^{(2)}$ & $C_{8}^{(4)}$ \\
\hline $\mathrm{He}-\mathrm{O}_{2}$ & 7.05 & 1.67 & 142.2 & 134.2 & 70.0 \\
$\mathrm{Na}-\mathrm{Na}_{2}$ & 2701.8 & 512.8 & 328170 & 157820 & 129350 \\
$\mathrm{~K}-\mathrm{K}_{2}$ & 4786.1 & 1540.4 & 518920 & 394400 & 260420 \\
\hline \hline
\end{tabular}

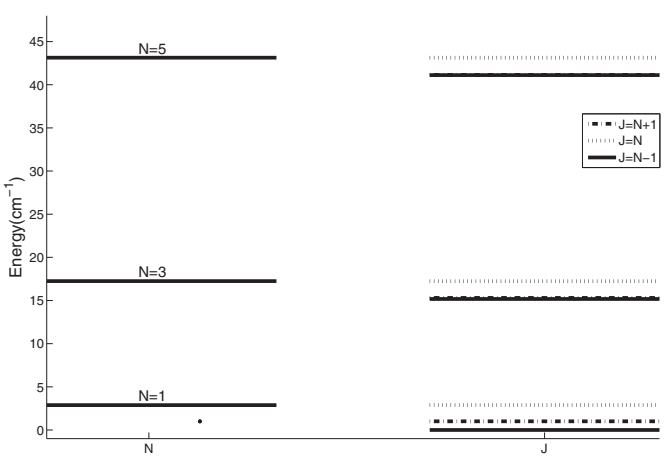

FIG. 2. Rotational structure for the $\mathrm{O}_{2}$ electronic ground state, $v=0$. Values for the fine structure are from [30]. The energy difference between some of the levels are too small to be fully resolved in the figure.

approaching at an angle $\theta=0^{\circ}$. Least repulsion is experienced for $\theta=90^{\circ}$, where the PES is also deepest with a maximum depth of $110 \mu E_{h}$ for $r=5.95$ a.u.

The PES is expanded in Legendre polynomials; cf. Eq. (6). The functions $v_{l}(r)$ are easily found by using the orthogonality relation for the Legendre polynomials and by performing the relevant integrals numerically. The functions $v_{0}(r)-v_{10}(r)$ are shown in Fig. 3.

Figure 3 reveals that the isotropic term $v_{0}(r)$ dominates over the anisotropic terms. The PES is in fact quite isotropic which can also be seen from Table IV; the anisotropic effects present in the PES are largely carried by the terms $v_{2}(r)$ and $v_{4}(r)$. The long range part of the potential becomes more important as the temperature decreases, and for ultracold temperatures it is necessary to integrate the coupled equations [Eq. (3)] to large values of $r$. We have integrated from $r=5$ a.u. out to $r=2000$ a.u. to ensure proper convergence of the cross sections. For temperatures around $1 \mu \mathrm{K}$, only the $L=0$ partial wave ( $s$ wave) contributes. To obtain a satisfactory convergence of the cross sections we include an increasing number of partial waves with increasing temperature. For the highest calculated temperature, $20 \mathrm{~K}$, we include all partial waves $L=0-35$ to achieve sufficient convergence of the cross sections. Both closed and open channels were included, this meaning that the total number of channels involved exceeds 1000 .

\section{A. Cross sections}

Here we report both elastic and inelastic cross sections for $\mathrm{O}_{2}$-He collisions. We consider $\mathrm{O}_{2}$ molecules in the $N=1$ rotational level as well as the excited $N=3$ level. The state to state cross sections we present may be relevant for magnetic trapping, thus we have included the different Zeeman levels $M_{j}$. See [15] or [10] for details on how to convert the $S$-matrix to the appropriate basis in which cross sections involving magnetic quantum numbers can be computed. Cross sections for $N=1$ at temperatures $T=10^{-6}-10 \mathrm{~K}$ are shown in Fig. 4. 
TABLE IV. Calculated PES for $\mathrm{O}_{2}$-He. Distances $r$ in a.u. and angles $\theta$ in degrees. Energies in $\mu E_{h}$. We have used the basis set aug-cc-pCVTZ [21-23] for both the $\mathrm{O}_{2}$ atom and the He atom. All values obtained with the quantum chemistry package GAMESS [18].

\begin{tabular}{ccccccccccc}
\hline \hline & & \multicolumn{1}{c}{$\theta$} & \multicolumn{1}{c}{$\theta$} & & & & \\
$r$ & 0 & 10 & 20 & 30 & 40 & 50 & 60 & 70 & 80 & 90 \\
\hline 5.00 & 5496 & 5324 & 4854 & 4148 & 3266 & 2327 & 1401 & 839.1 & 450.7 & 321.2 \\
5.50 & 1466 & 1429 & 1314 & 1123 & 867.6 & 585.9 & 326.6 & 127.1 & 5.878 & -34.53 \\
6.00 & 271.8 & 267.8 & 250.3 & 212.7 & 151.7 & 76.55 & 3.967 & -53.53 & -89.34 & -107.4 \\
6.50 & -40.28 & -37.42 & -24.72 & -21.21 & -22.77 & -36.94 & -58.10 & -77.89 & -91.42 & -95.99 \\
7.00 & -94.86 & -92.18 & -85.14 & -76.74 & -70.79 & -68.53 & -68.84 & -69.92 & -71.26 & -71.19 \\
8.00 & -60.75 & -59.48 & -56.15 & -51.85 & -47.28 & -43.03 & -39.45 & -36.74 & -35.12 & -34.59 \\
9.00 & -29.65 & -29.26 & -27.83 & -26.07 & -23.85 & -21.73 & -19.85 & -18.26 & -17.12 & -17.02 \\
10.0 & -14.88 & -14.65 & -14.08 & -13.27 & -12.26 & -11.23 & -10.30 & -9.525 & -9.045 & -8.892 \\
11.0 & -7.956 & -8.020 & -7.584 & -7.165 & -6.665 & -6.142 & -5.655 & -5.270 & -5.119 & -4.939 \\
12.0 & -4.532 & -4.515 & -4.278 & -4.106 & -2.330 & -3.548 & -3.945 & -3.076 & -2.969 & -2.894 \\
14.0 & -1.704 & -1.674 & -1.548 & -1.548 & -1.847 & -1.362 & -1.278 & -1.190 & -1.030 & -1.128 \\
16.0 & -0.7425 & -0.7145 & -0.6808 & -0.6437 & -0.6281 & -0.5951 & -0.5558 & -0.5280 & 0.5000 & -0.5011 \\
\hline \hline
\end{tabular}

The cross sections are calculated at zero magnetic field. From a computational point of view, zero magnetic field is convenient as the different $\mathcal{J}$ values do not interact and may be computed separately. The cross sections will of course be somewhat modified by the presence of a magnetic field, in particular the energies at which different resonances occur. The resonance seen in Fig. 4 at $T \simeq 0.9 \mathrm{~K}$ will most certainly reassert itself at a different energy in a magnetic field, although we expect the general behavior of the cross sections to be similar. Even though a magnetic field may alter the cross sections, we comment below on their relevance for magnetic trapping. At the center of a magnetic trap, the magnetic field will be small or simply vanish. Thus the perturba- tion on the system caused by the magnetic field should yield only small changes in the overall behavior of the cross sections, in particular for geometries close to the center of the trap.

It is evident from Fig. 4 that the inelastic cross sections are several orders of magnitudes smaller than the elastic cross sections. This is certainly encouraging for experiments that involve buffer-gas cooling of magnetically trapped $\mathrm{O}_{2}$ molecules. In particular for low temperatures elastic scattering is seen to be by far dominating (in the lowest rotational level), thus minimizing the number of collisions between $\mathrm{O}_{2}$ molecules and $\mathrm{He}$ atoms that lead to trap loss. One also needs to be aware that inelastic scattering does not necessar-

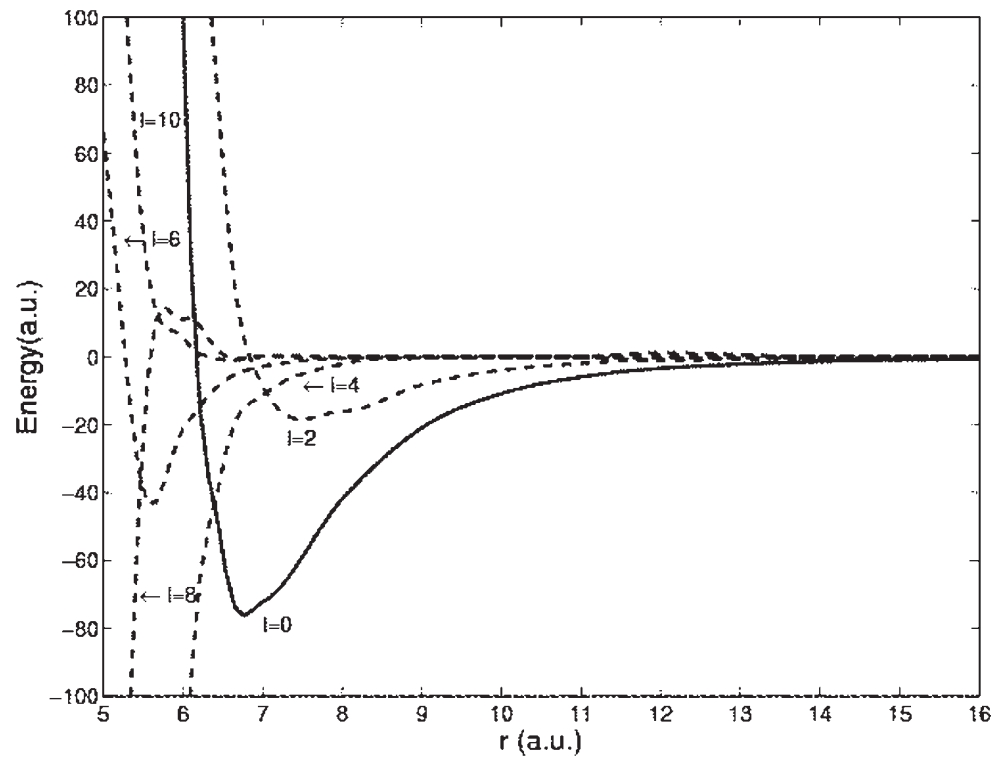

FIG. 3. $v_{l}(r)$ for $l$ $=0,2,4,6,8,10 . v_{l}(r)$ is zero for odd $l$ since the PES is symmetric about $\theta=90^{\circ}$. The isotropic contribution is plotted as a solid line. 


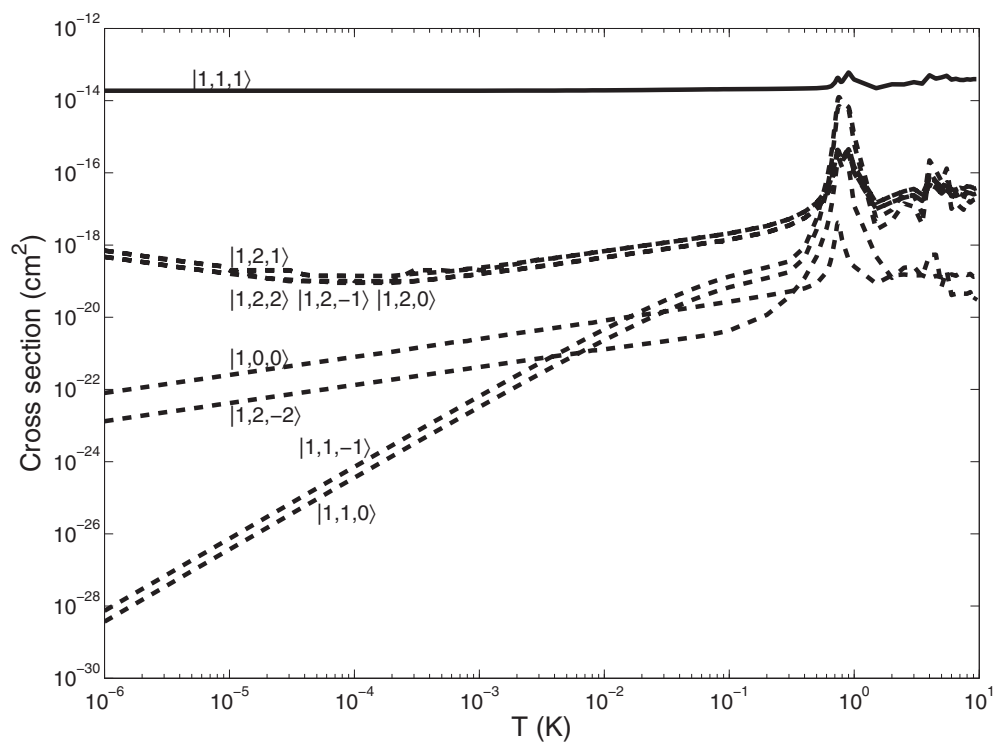

FIG. 4. Cross sections for both elastic (solid line) and inelastic scattering (dashed lines) $\left|N, J, M_{J}\right\rangle \rightarrow\left|N^{\prime}, J^{\prime}, M_{J}^{\prime}\right\rangle$ for $\quad N$ $=1, J=1, M_{J}=1$. Away from any resonances the elastic scattering process is seen to be dominating in the $N=1$ rotational level. ily result in trap loss as the final channel may involve $M_{J}$ quantum numbers larger than zero, making the final state magnetically trapable. In Fig. 5 we give the partial waves $L=0,1,2$ for the elastic scattering process $\mid N=1, J=1, M_{J}$ $=1\rangle \rightarrow|1,1,1\rangle$. The Wigner threshold law [32] (for elastic scattering)

$$
\sigma_{\mathrm{el}} \propto E^{2 L}
$$

can be seen to be obeyed in Fig. 5 for temperatures $T$ $<10^{-3}$ K. From Fig. 5 it is also evident that partial waves $L>0$ give only a negligible contribution to the total cross section for temperatures in the $\mathrm{mK}$ range.

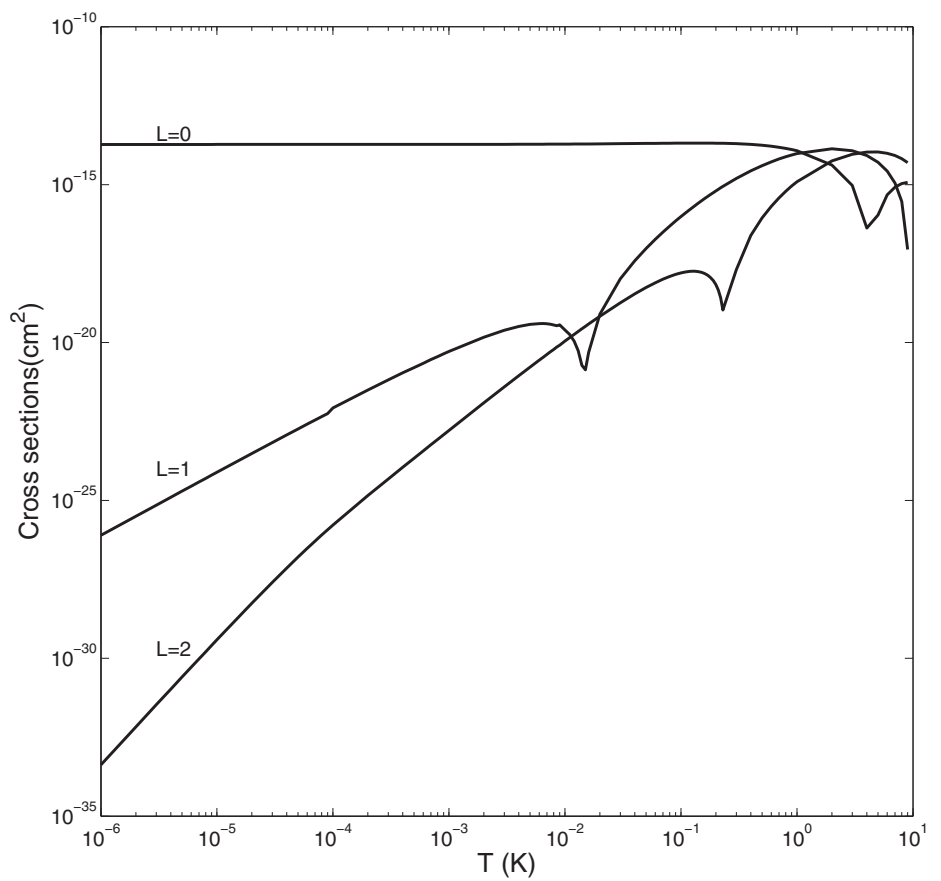

FIG. 5. Contributions to elastic cross sections from partial waves $L=0,1,2$ as a function of temperature. The partial wave cross sections follow the Wigner threshold law. 


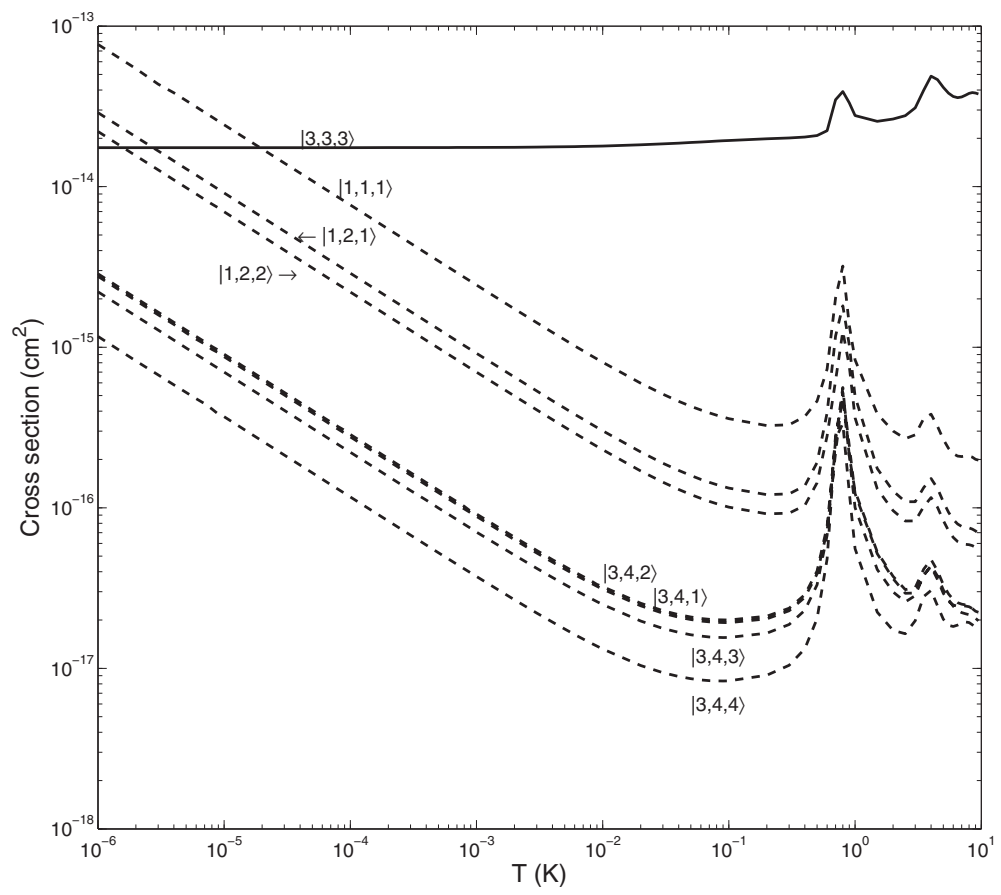

FIG. 6. Cross sections for $\left|N, J, M_{J}\right\rangle \rightarrow\left|N^{\prime}, J^{\prime}, M_{J}^{\prime}\right\rangle$ with $N$ $=3, J=3, M_{J}=3$. Cross sections between states not shown in the figure are negligible. Solid line represents elastic scattering.

In addition we have calculated cross sections for rotationally excited molecules in the $N=3$ rotational level. Accurate calculations of the different inelastic cross sections from excited rotational levels are computationally more demanding, as there are many more partial waves that need to be included even at low energies. We show cross sections for molecules initially in the $|3,3,3\rangle$ state (see Fig. 2) in Fig. 6. The elastic cross section is calculated to be of the order $10^{-14} \mathrm{~cm}^{2}$ and is largely temperature independent away from any resonances, with a slight increase for larger temperatures. The inelastic cross sections are again several orders of magnitudes smaller for temperatures around $1 \mathrm{~K}$; thus rotational cooling as a result of collisions with $\mathrm{He}$ is a very limited possibility. The inelastic cross sections are further seen to increase rapidly as the temperature is lowered. Cooling of the translational degree of freedom by elastic collisions may therefore result in more efficient rotational cooling, since the inelastic cross sections for scattering $N=3$ $\rightarrow N=1$ increases as the temperature is lowered. The behavior of the inelastic cross sections in Fig. 6 can be understood from the Wigner threshold law [32] for inelastic scattering

$$
\sigma_{\text {inelastic }} \propto E^{L-1 / 2},
$$

with $L=0$. A prominent feature in Fig. 6 is the appearance of a resonance at an energy corresponding to $T \simeq 0.8 \mathrm{~K}$. This represents a shape resonance in the $L=3$ partial wave, confirmed by calculating the elastic partial wave cross sections with only the isotropic part of the potential included: $V(r, \theta)=v_{0}(r)$.
As previously mentioned, evaporative cooling is needed to reach Bose-Einstein relevant temperatures. However, our calculations cannot be used to predict how efficient $\mathrm{O}_{2}$ can be evaporatively cooled as this would require knowledge of the $\mathrm{O}_{2}-\mathrm{O}_{2}$ cross sections. As far as the authors are aware, cross sections for $\mathrm{O}_{2}-\mathrm{O}_{2}$ have never been calculated due to the complexity of the intramolecular interaction.

We have however studied the buffer cooling process in some detail, based on the cross sections we have calculated. In Table $\mathrm{V}$ we report selected calculated cross sections for scattering between the different rotational levels, which are important for the next subsection on buffer-gas cooling.

\section{B. Buffer-gas cooling}

Diatomic molecules have noncentral interactions [cf. Eq. (20)] with the buffer gas, thus it is possible to cool both the translational and the rotational degree of freedom. We have simulated the buffer-gas cooling process numerically based on our calculated cross sections $\sigma_{N \rightarrow N^{\prime}}$. For this purpose we have used the direct simulation Monte Carlo (DSMC) method $[33,34]$ with cross sections from our calculations. The DSMC method is developed from the physics of gas flow and has a close relationship with the Boltzmann equation. The philosophy of the DSMC method is to model a real gas flow with a relative small number of simulated molecules (in general particles). The simulated region in space (threedimensional) is divided into cells of equal size, with different numbers of molecules in each cell. Initially the molecules are distributed randomly with a random number generator. At 
TABLE V. Cross sections $\sigma_{N \rightarrow N^{\prime}}$ for the scattering processes $N \rightarrow N^{\prime}$ at selected temperatures. All values are given in $10^{-17} \mathrm{~cm}^{-2}$.

\begin{tabular}{ccccccccccc}
\hline \hline$T(\mathrm{~K})$ & $\sigma_{11}$ & $\sigma_{33}$ & $\sigma_{31}$ & $\sigma_{55}$ & $\sigma_{53}$ & $\sigma_{51}$ & $\sigma_{77}$ & $\sigma_{75}$ & $\sigma_{73}$ & $\sigma_{71}$ \\
\hline 0.01 & 1981 & 1791 & 134.6 & 1793 & 286.1 & 99.32 & 1839 & 112.0 & 44.27 & 10.11 \\
0.1 & 2077 & 1939 & 59.25 & 1930 & 117.5 & 42.81 & 1939 & 169.1 & 46.87 & 8.339 \\
1.0 & 3830 & 1448 & 170.9 & 2621 & 571.3 & 135.2 & 2622 & 721.7 & 45.51 & 7.101 \\
3.0 & 3239 & 1615 & 52.52 & 3044 & 128.5 & 36.41 & 3015 & 175.4 & 42.14 & 5.116 \\
5.0 & 4396 & 1992 & 46.17 & 3990 & 127.1 & 35.30 & 3173 & 175.1 & 41.83 & 3.971 \\
7.0 & 3745 & 2611 & 34.52 & 3542 & 90.46 & 25.70 & 3417 & 174.4 & 41.78 & 3.611 \\
9.0 & 3922 & 2554 & 31.58 & 3754 & 79.14 & 22.16 & 3771 & 151.8 & 39.40 & 3.402 \\
11.0 & 3568 & 2598 & 25.75 & 3467 & 84.55 & 19.91 & 3102 & 136.3 & 37.71 & 3.089 \\
13.0 & 3211 & 2689 & 23.95 & 3211 & 75.58 & 19.18 & 3615 & 130.9 & 35.51 & 3.012 \\
15.0 & 3121 & 2807 & 23.28 & 3415 & 70.84 & 17.54 & 3694 & 125.6 & 30.57 & 3.883 \\
17.0 & 3120 & 2808 & 21.25 & 3268 & 69.59 & 15.43 & 3667 & 123.6 & 28.87 & 3.339 \\
19.0 & 3063 & 2762 & 59.25 & 3178 & 66.14 & 16.99 & 3594 & 115.4 & 22.77 & 3.735 \\
\hline \hline
\end{tabular}

each time step the molecules are moved $\mathbf{r}_{i} \rightarrow \mathbf{r}_{i}+\mathbf{v}_{i} \Delta t$ and may of course in this process change cell. One of the key ideas to the method is the decoupling of translation and collision; the molecules are first moved without any interaction from other molecules or atoms. External forces, e.g., a magnetic field or gravity may be included in the moving step. Interaction with other molecules then proceeds in a collision step after the molecules are moved. At each time step some of the molecules are selected to collide and the outcome of these collisions depends on the different cross sections. The energy in each collision is found from the relative velocity and the cross section is estimated from an interpolation in a table of calculated cross sections as a function of energy. Throughout the process each molecule is monitored and information about velocity and rotational levels are updated at every time step. The number of simulated collisions $N_{\text {coll }}$ in a cell during a timestep $\Delta t$ is determined by kinetic theory and is given by

$$
N_{\text {coll }}=\frac{N_{c}\left(N_{c}-1\right) \sigma v_{\max } \Delta t}{2 V_{c}},
$$

where $N_{c}$ is the number of molecules in a given cell and $V_{c}$ is the volume of that cell. $v_{\max }$ is an upper limit for the relative velocities. We have used bounce-back (reflecting walls) boundary conditions in the simulations.

For the DSMC method to be reliable the time step $\Delta t$ must be chosen in such a way that it is smaller than or of the same order as the mean collision time $\tau$. Correspondingly, the cell length should be smaller than the mean free path. The physical parameters used in our simulations yield a mean collision time $\tau=4.2 \times 10^{-4} \mathrm{~s}$ and a mean free path $l$ $=1.3 \times 10^{-2} \mathrm{~cm}$.

For our simulation to be as realistic as possible we have assumed a buffer-gas density of $10^{15} \mathrm{~cm}^{-3}$. In the simulations we use $10^{6} \mathrm{He}$ atoms and $2000 \mathrm{O}_{2}$ molecules. The physical size of the box is determined from the density and number of atoms and molecules. The He atoms are initially at a temperature $T=1.0 \mathrm{~K}$, while the initial velocity distribution for the $\mathrm{O}_{2}$ molecules is a delta function with a velocity corresponding to the temperature $T=20 \mathrm{~K}$, estimated from the relation $E=\frac{3}{2} k_{B} T$.

Our simulations only account for collisions between the He buffer-gas and the $\mathrm{O}_{2}$ molecules. Collisions among the $\mathrm{O}_{2}$ molecules themselves should be rare, whereas collisions between $\mathrm{He}$ atoms are rather uninteresting. Initially, the rotational quantum numbers for the $\mathrm{O}_{2}$ molecules are distributed according to the Boltzmann distribution; see Fig. 7.

After simulation of the buffer gas cooling process for some time we find that the $\mathrm{O}_{2}$ molecules have been thermalized to a temperature close to the buffer-gas temperature. This is confirmed by plotting the speed distribution together with the Maxwell speed distribution $\mathcal{D}(v)$ for $T=1.0 \mathrm{~K}$, to observe the excellent agreement. Upon thermalization we also observe the mean speed of the $\mathrm{O}_{2}$ molecules fluctuate around a mean value.

Figure 8 shows plots of the results of the thermalization process. The (mean) number of collisions per molecule are $8.22,14.7,21.5$, and 28.7 corresponding to the times $t$

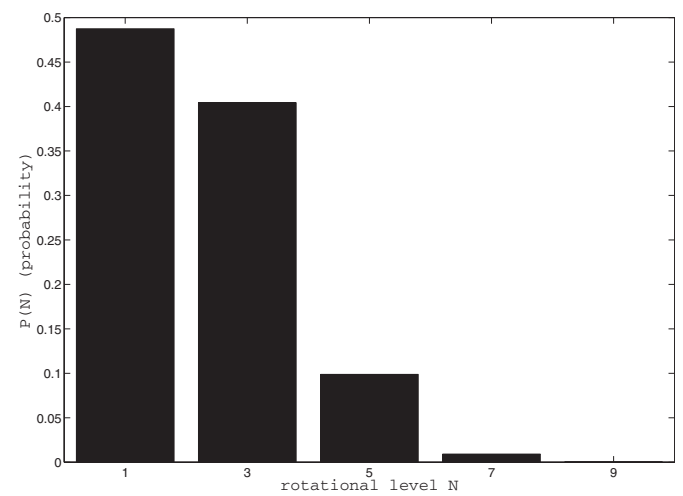

FIG. 7. Probability distribution for different rotational levels $N$ in $\mathrm{O}_{2}$ at $T=20 \mathrm{~K}$. 

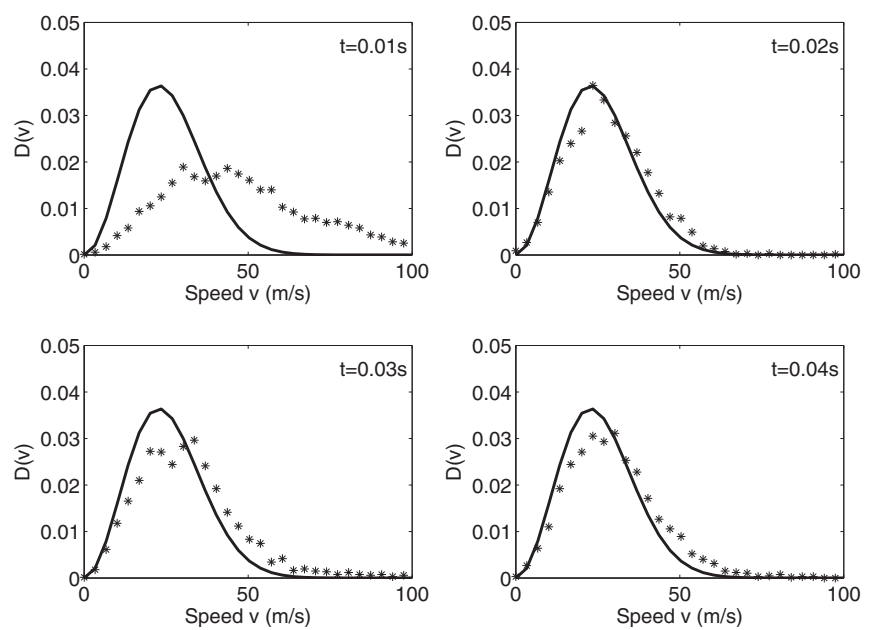

FIG. 8. Speed distribution for $\mathrm{O}_{2}$ molecules at times $t=0.01 \mathrm{~s}$, $0.02 \mathrm{~s}, 0.03 \mathrm{~s}$, and $0.04 \mathrm{~s}$ (asterisks), compared to the Maxwell distribution (sold line) plotted at $T=1 \mathrm{~K}$.

$=0.01,0.02,0.03$, and $0.04 \mathrm{~s}$. We conclude that thermalization of the translational degree of freedom for $\mathrm{O}_{2}$ molecules initially at $T=20 \mathrm{~K}$ with a buffer gas at $1 \mathrm{~K}$ requires the $\mathrm{O}_{2}$ molecules undergo roughly 20 collisions with $\mathrm{He}$.

In our simulations we have also included collisions in which the rotational quantum number $N$ is changed. In a collision with energy $E$ we calculate the probabilities for an allowed transition based on the cross sections. A random number is then generated to determine the outcome of the collision. We do allow for rotational excitations provided that the energy involved is sufficient. It is easily seen from the cross sections that most collisions will be elastic and this should be expected from the calculated PES which is quite isotropic.

Due to the small probabilities for change of the rotational level in a collision, the rotational degree of freedom requires many more collisions to attain a population typical for $1 \mathrm{~K}$ (99.9\% in $N=1$ according to the Boltzmann distriubution). In Fig. 9 we show the rotational population numbers after times $t=0,0.03,0.1$, and $0.5 \mathrm{~s}$. The initial distribution of rotational levels for the $\mathrm{O}_{2}$ molecules follow the Boltzmann distribution for $T=20 \mathrm{~K}$ (see Fig. 7).

The efficiency of translational and rotational cooling may be compared most easily by the number of collisions required to reach equilibrium. Whereas cooling of the translational freedom is seen to require 15-20 collisions with the buffer gas, the rotational distribution has still not reached an equilibrium distribution after 71 collisions.

\section{V. $\mathrm{Na}_{2}-\mathrm{Na}$ SYSTEM}

We now turn our attention to $\mathrm{Na}$ and study collisions between ${ }^{23} \mathrm{Na}_{2}$ molecules and $\mathrm{Na}$ atoms. The $\mathrm{Na}_{2}$ internuclear separation is kept fixed at $r_{e}=3.0786 \AA$. The $\mathrm{Na}_{2}$ ground state, ${ }^{1} \Sigma_{g}^{+}$, has neither spin nor angular momenta. $\mathrm{Na}_{2}$ molecules are usually cooled with other cooling methods apart from buffer-gas cooling. Since the ground state is not magnetically trapable it is also challenging to employ the evapo- rative cooling technique. Ultracold ground state molecules may instead be a likely result of molecule formation in photoassociation experiments. A two color photoassociation scheme is one example of a cooling method likely to yield ground state $\mathrm{Na}_{2}$ molecules residing in the lowest attainable vibrational level $(v=0)$ [7]. These ultracold ground state molecules will collide with $\mathrm{Na}$ atoms not yet photoassociated to a an excited electronic state. As both molecule and atom have temperatures in the $\mu \mathrm{K}$ temperature range, the collisions between them will necessarily be cold. We have computed cross sections for both elastic and inelastic collisions at temperatures ranging from $10^{-9} \mathrm{~K}$ to $10^{-2} \mathrm{~K}$, as this should

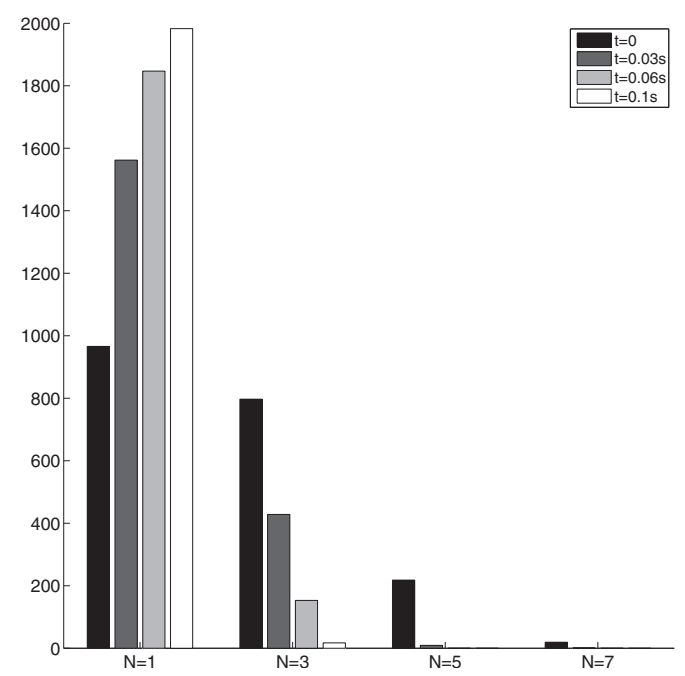

FIG. 9. Rotational distribution for $\mathrm{O}_{2}$ molecules after $0(t=0)$, $21(t=0.03 \mathrm{~s}), 42(t=0.06 \mathrm{~s})$, and $71(t=0.1 \mathrm{~s})$ collisions with the He buffer gas. 
TABLE VI. Calculated PES for the $\mathrm{Na}_{2}$-Na interaction. Distances $r$ in a.u. and angles $\theta$ in degrees. Energies are given in $\mathrm{m} E_{h}$. We use the basis set cc-pCTZ [22] for all atoms involved in the calculation.

\begin{tabular}{|c|c|c|c|c|c|c|c|c|c|c|}
\hline \multirow[b]{2}{*}{$r$} & \multicolumn{10}{|c|}{$\theta$} \\
\hline & 0 & 10 & 20 & 30 & 40 & 50 & 60 & 70 & 80 & 90 \\
\hline 4.00 & 2742 & 2088 & 1027 & 425.9 & 203.8 & 142.45 & 30.65 & 24.80 & 21.51 & 18.98 \\
\hline 5.00 & 837.3 & 656.1 & 353.9 & 167.6 & 85.12 & 51.24 & 12.30 & 5.110 & 2.968 & 2.927 \\
\hline 6.00 & 122.1 & 108.3 & 78.49 & 48.89 & 25.84 & 10.33 & 1.314 & -3.07 & -3.912 & -3.460 \\
\hline 7.00 & 33.01 & 29.66 & 21.38 & 11.89 & 4.068 & -1.007 & -3.570 & -4.389 & -4.316 & -4.182 \\
\hline 8.00 & 6.008 & 5.189 & 2.968 & 0.4552 & -1.723 & -3.139 & -3.606 & -3.535 & -3.333 & -3.240 \\
\hline 9.00 & -0.4639 & -0.7722 & -1.528 & -2.318 & -2.797 & -2.884 & -2.709 & -2.461 & -2.271 & -2.202 \\
\hline 10.0 & -2.406 & -2.444 & -2.507 & -2.494 & -2.347 & -2.101 & -1.836 & -1.621 & -1.487 & -1.443 \\
\hline 11.0 & -2.223 & -2.186 & -2.070 & -1.879 & -1.640 & -1.400 & -1.194 & -1.050 & -0.9671 & -0.9402 \\
\hline 12.0 & -1.573 & -1.531 & -1.415 & -1.247 & -1.064 & -0.8960 & -0.7653 & -0.6776 & -0.6288 & -0.6133 \\
\hline 13.0 & -1.010 & -0.9800 & -0.8992 & -0.7881 & -0.6712 & -0.5679 & -0.4892 & -0.4374 & -0.4093 & -0.4006 \\
\hline 14.0 & -0.6249 & -0.6066 & -0.5574 & -0.4907 & -0.4214 & -0.3606 & -0.3142 & -0.2837 & -0.2673 & -0.2623 \\
\hline 15.0 & -0.3843 & -0.3737 & -0.3453 & -0.3067 & -0.2665 & -0.2312 & -0.2039 & -0.1857 & -0.1759 & -0.1728 \\
\hline 16.0 & -0.2389 & -0.2328 & -0.2166 & -0.1944 & -0.1711 & -0.1504 & -0.1343 & -0.1233 & -0.1173 & -0.1153 \\
\hline
\end{tabular}

be the most relevant temperature range. In the calculations we have included partial waves up to $L=25$.

In Table VI we list the short range PES calculated for $r$ $=4.00$ a.u. to $r=16.0$ a.u.. For $r>16.0$ a.u. we use Eq. (20) with coefficients from Table IV. Small inconsistencies between the long-range PES and the GAMESS PES are resolved by making a smooth transition between the two with a polynomial fit. We do believe that our dispersion coeffi- cients give the most accurate description of the PES at $r$ $=16$ a.u.; thus we have matched the GAMESS PES to the long range PES calculated from dispersion coefficients. Again we have integrated the close coupled Eqs. (3) out to $r=2000$ a.u., starting from $r=4.0$ a.u.

In Fig. 10 we give $v_{l}(r)$ for the calculated PES. Whereas the $\mathrm{O}_{2}-\mathrm{He}$ interaction was given in $\mu E_{h}$, the $\mathrm{Na}_{2}-\mathrm{Na}$ interaction is reported in $m E_{h}$. This increased interaction energy is

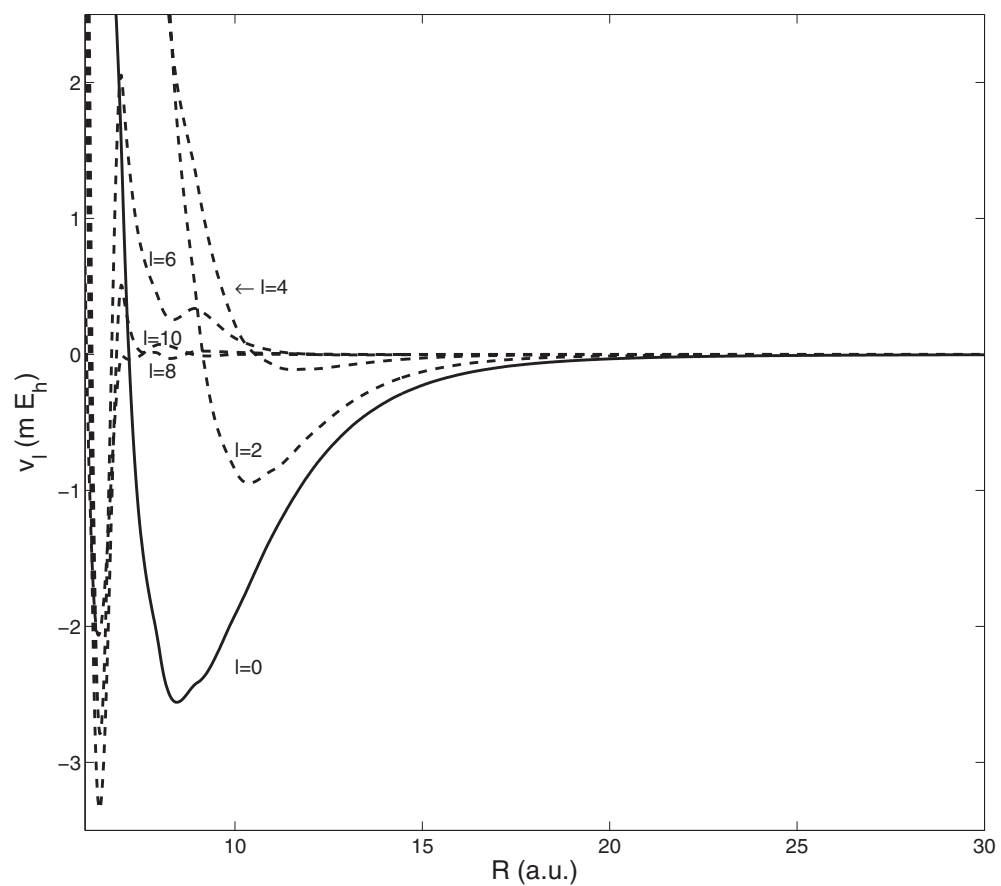

FIG. 10. Different terms $v_{l}(r)$ [cf. Eq. (6)] for the $\mathrm{Na}_{2}-\mathrm{Na}$ interaction. 


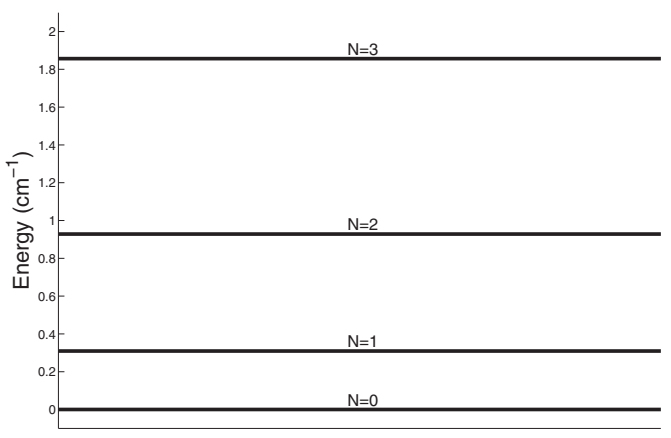

FIG. 11. Rotational energies for the $N=0,1,2,3$ rotational levels in the ground electronic and vibrational state of the $\mathrm{Na}_{2}$ molecule.

largely due to the valence electron of $\mathrm{Na}$. It is also evident from Fig. 10, that the larger contributions stem from $v_{0}(r)$, $v_{2}(r), v_{4}(r)$, and $v_{6}(r) . v_{8}(r)$ and $v_{10}(r)$, on the other hand, provide only small corrections. The anisotropic terms in the PES, $v_{2}(r)$ and $v_{4}(r)$, are largely responsible for the inelastic scattering between different rotational levels. By comparing Fig. 10 to Fig. 4, the anisotropic terms can be seen to give a larger contribution for the $\mathrm{Na}_{2}-\mathrm{Na}$ system than for the $\mathrm{O}_{2}-\mathrm{He}$ system. We would thus expect a larger fraction of the total cross section to be inelastic.

All rotational levels are allowed in the ${ }^{23} \mathrm{Na}_{2}$ molecule as the nuclear spin of the $\mathrm{Na}$ atom is $I=\frac{3}{2}$. Due to the small rotational constant $B_{e}=0.15471 \mathrm{~cm}^{-1}$, the rotational levels are packed close together; see Fig. 11. The molecular spin is zero $(\mathbf{S}=0)$; thus $\mathbf{N}=\mathbf{J}$ and there is no fine structure in the ground state. The $\mathrm{Na}$ atom do however have an open shell, giving a $\operatorname{spin} \mathbf{s}=\frac{1}{2}$.
Inelastic scattering processes are only allowed between rotational levels with the same parity. This is a direct consequence of the fact that the $\mathrm{Na}_{2}$ molecule is homonuclear with only even values of $l$ allowed in the sum in Eq. (6). For the $3 j$ symbol involving $N$ and $N^{\prime}$ in Eq. (9) to be nonzero, $N$ $+N^{\prime}$ must be an even number. This makes transitions between neighboring rotational levels forbidden, making it impossible for molecules in the $N=1$ rotational level to reach the $N=0$ level through collisions with $\mathrm{Na}$.

The inelastic and elastic cross sections for the four lowest rotational levels are shown in Fig. 12. The cross sections are seen to be several magnitudes larger than for the $\mathrm{O}_{2}$-He system; this should not be surprising as $\mathrm{Na}$ is heavier and extends further in space than both the $\mathrm{O}$ and the He atoms. The alkalis are further known to have large cross sections, which is one of the reasons why Bose-Einstein condensates were first obtained with alkalis and not with hydrogen.

The elastic cross sections for the different rotational levels are all of similar size and show many of the same features. Away from any resonances, at $\mathrm{mK}$ temperatures, the elastic cross sections are in fact almost identical. This should probably be expected as the rotational constant is small; the energy difference between the rotational levels become more and more negligible as the temperature increases.

The inelastic cross sections do of course increase at low temperatures, as predicted by the Wigner threshold. It is interesting to see that the inelastic cross section, in particular for the $N=3 \rightarrow N^{\prime}=1$ transition, completely dominates over the elastic $N=3 \rightarrow N^{\prime}=3$ transition for temperatures $T$ $<1 \mu \mathrm{K}$. This indicates that very efficient rotational cooling can be achieved for collisions at these low temperatures. At $\mathrm{mK}$ temperatures, the inelastic cross sections are roughly a factor $\frac{1}{40}$ of the elastic ones; thus the possibility of rotational cooling is severely restricted. As we will see, the situation is quite similar for the $\mathrm{K}_{2}-\mathrm{K}$ system.

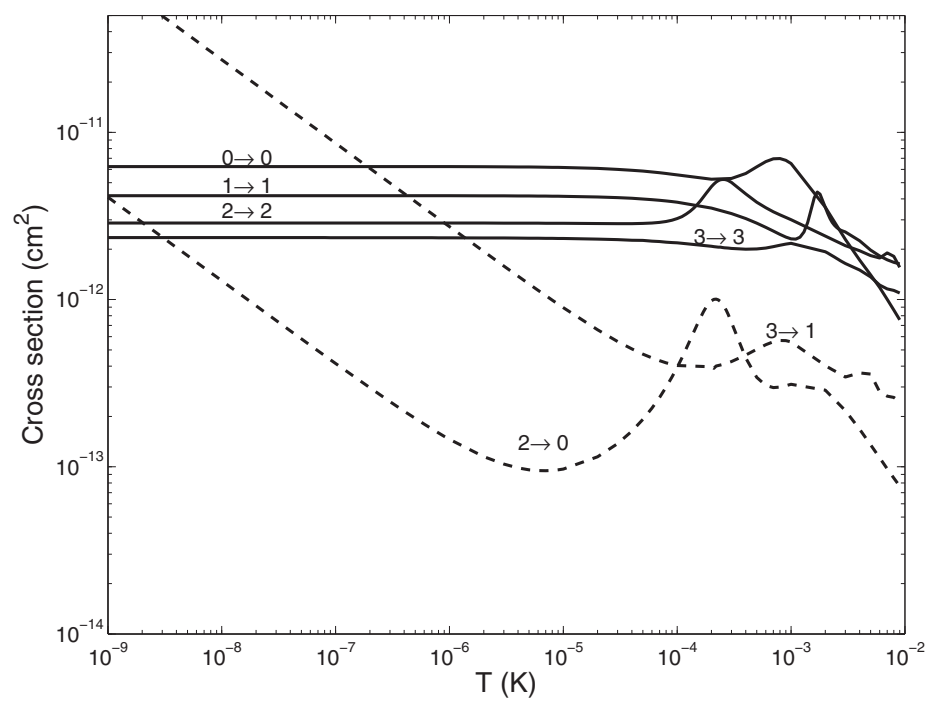

FIG. 12. Elastic and inelastic cross sections for $N=0,1,2,3$ rotational levels in the ground electronic and vibrational state of $\mathrm{Na}_{2}$. Elastic cross sections are plottes as solid lines. 
TABLE VII. Calculated PES for the $\mathrm{K}_{2}-\mathrm{K}$ interaction. Distances $r$ in a.u. and angles $\theta$ in degrees. Energies are given i $\mathrm{m} E_{h}$. We have used the triple zeta valence (TZV) [23] basis set augmented with polarization functions of type $d$.

\begin{tabular}{|c|c|c|c|c|c|c|c|c|c|c|}
\hline \multirow[b]{2}{*}{$r$} & \multicolumn{10}{|c|}{$\theta$} \\
\hline & 0 & 10 & 20 & 30 & 40 & 50 & 60 & 70 & 80 & 90 \\
\hline 6.00 & 2351 & 1844 & 966.0 & 396.8 & 155.9 & 70.76 & 38.78 & 24.20 & 21.92 & 15.65 \\
\hline 7.00 & 437.3 & 360.6 & 214.8 & 108.7 & 55.42 & 29.80 & 15.84 & 8.148 & 4.828 & 10.97 \\
\hline 8.00 & 99.89 & 87.63 & 61.94 & 38.50 & 21.70 & 10.40 & 3.418 & -0.2382 & -1.475 & 4.836 \\
\hline 9.00 & 33.29 & 30.03 & 22.16 & 13.22 & 5.619 & 0.3255 & -2.710 & -4.016 & -4.158 & 2.138 \\
\hline 10.0 & 9.678 & 8.319 & 4.948 & 1.048 & -2.169 & -4.218 & -5.188 & -5.394 & -5.134 & 1.025 \\
\hline 11.0 & -1.018 & -1.539 & -2.809 & -4.213 & -5.260 & -5.794 & -5.898 & -5.735 & -1.947 & 0.5370 \\
\hline 12.0 & -5.087 & -5.238 & -5.586 & -5.922 & -6.098 & -6.085 & -5.939 & -3.612 & 0.2887 & 0.2950 \\
\hline 13.0 & -6.140 & -6.158 & -6.187 & -6.177 & -6.102 & -5.969 & -3.612 & 0.1240 & 0.1548 & 0.1643 \\
\hline 14.0 & -6.139 & -6.126 & -6.084 & -6.011 & -4.782 & -0.07322 & 0.007790 & 0.05768 & 0.08340 & 0.09118 \\
\hline 15.0 & -4.783 & -4.813 & -4.894 & -0.2582 & -0.1503 & -0.06121 & -0.007089 & 0.02748 & 0.04485 & 0.05003 \\
\hline 16.0 & -0.3229 & -0.3007 & -0.2425 & -0.1684 & -0.09840 & -0.04397 & -0.007683 & 0.01356 & 0.02403 & 0.02712 \\
\hline 17.0 & -0.1912 & -0.1778 & -0.1431 & -0.09899 & -0.05763 & -0.02587 & -0.008761 & 0.006983 & 0.01283 & 0.01540 \\
\hline 18.0 & -0.1054 & -0.09793 & -0.07850 & -0.05401 & -0.03121 & -0.03289 & -0.01034 & 0.003745 & 0.006824 & 0.007716 \\
\hline 19.0 & -0.05487 & -0.05092 & -0.04067 & -0.04830 & -0.02910 & -0.03129 & -0.01238 & 0.001454 & 0.003613 & 0.004059 \\
\hline 20.0 & -0.04821 & -0.04551 & -0.03771 & -0.04445 & -0.02832 & -0.028760 & -0.01436 & 0.001157 & 0.001900 & 0.002113 \\
\hline
\end{tabular}

\section{K K$_{2}$-K SYSTEM}

We now turn our attention to a heavier alkali system, namely the ${ }^{39} \mathrm{~K}_{2^{-}}{ }^{39} \mathrm{~K}$ system. As $\mathrm{K}$ and $\mathrm{Na}$ have many of the chemical properties in common the cross sections may be expected to behave similarly.

Both species have attracted considerable interest in recent years, and translationally ultracold samples of both $\mathrm{Na}_{2}$ and $\mathrm{K}_{2}$ molecules may nowdays be produced in the laboratory. As $\mathrm{K}$ is heavier than $\mathrm{Na}$ it is also more challenging to reliably calculate the interaction between the $\mathrm{K}_{2}$ molecules and the $\mathrm{K}$ atom. Here we report cross sections obtained with the PES calculated with GAMESS at short range and by using the dispersion coefficients (see Sec. III) to reliably model the long range potential. The short range PES obtained is reported in Table VII for $r \in[6.00-20.00$ a.u.].

For all geometries the Hartree-Fock calculations are convergent $\left(\Delta E<10^{-6}\right.$ a.u. $)$, although at some geometries the convergence was rather slow. Indeed this was the case for some geometries $\left(\theta=0-50^{\circ}\right)$ at $r=15$ a.u. and for a few other spread geometries. The problem is most easily solved by simply performing an additional number of Hartree-Fock iterations or by using a level shift. That is, lifting the diagonal elements of the Fock matrix up (in energy) to uncouple the occupied and unoccupied orbitals. This is a clear indication that the slow convergence stems from electronic states which lie close together, although not exactly degenerate, at least not at the geometries we have investigated. This problem is of course also encountered for atom-atom collisions, although more frequently for atom-molecule collisions as the number of electronic states are much larger.

The internuclear distance for the $\mathrm{K}_{2}$ molecule was kept at the equilibrium distance of $r_{e}=3.923 \AA$. At $r=20$ a.u. we connect the short-range PES to the long-range PES. Additional consideration is needed to do this as the short-range
PES is in fact repulsive for some of the larger values of $\theta$ even at $r=20$ a.u., whereas the long-range PES of course is attractive [cf. Eq. (20)]. As a solution we make a smooth transition between the two energy surfaces for $r$ $=20-25$ a.u. . For $r>25$ a.u. we use solely the long range PES. This inconsistency is of course evidence of inaccuracies in one or both of the calculated surfaces. As we do believe the long-range PES is the most reliable of the two surfaces, we have modified the short range PES to match the long range PES at $r=25$ a.u.

The $\mathrm{K}_{2}-\mathrm{K}$ PES is expanded in Legendre polynomials [cf. Eq. (6)]. As the PES is quite anisotropic we have needed to include Legendre polynomials for $l \in[0,40]$ to ensure a representation of the PES with at least two leading digits of accuracy for all geometries. Figure 13 gives the first functions $v_{l}$ for $l=0-10$ and should be compared to Fig. 10 . The strong anisotropy in the PES (Table VII) affects the behavior of the plotted terms $v_{l}(r)$; all terms yield significant contributions.

$\mathrm{K}_{2}$ molecules have a ground state rotational constant $B_{e}$ $=0.05622 \mathrm{~cm}^{-1}$ with a rotational structure as shown in Fig. 14. We give the cross sections for the lowest rotational levels, of relevance for cold and ultracold collisions.

From Fig. 15 we may note the following. (1) The elastic cross section is a factor 10 larger for elastic collisions $N=1$ $\rightarrow N^{\prime}=1$ than for any other elastic collision process at ultralow energies. The $N=1 \rightarrow N^{\prime}=1$ elastic cross section is several orders of magnitude larger than the $N=0 \rightarrow N^{\prime}=0$ elastic cross section. (2) For temperatures in the $\mathrm{mK}$ range, away from any resonances, the different elastic cross sections are of equal magnitude, whereas inelastic collisions are suppressed. (3) The Wigner threshold law is obeyed at ultralow energies.

Cross sections are known to be very sensitive for changes in the scattering potential, in particular at low energies. To 


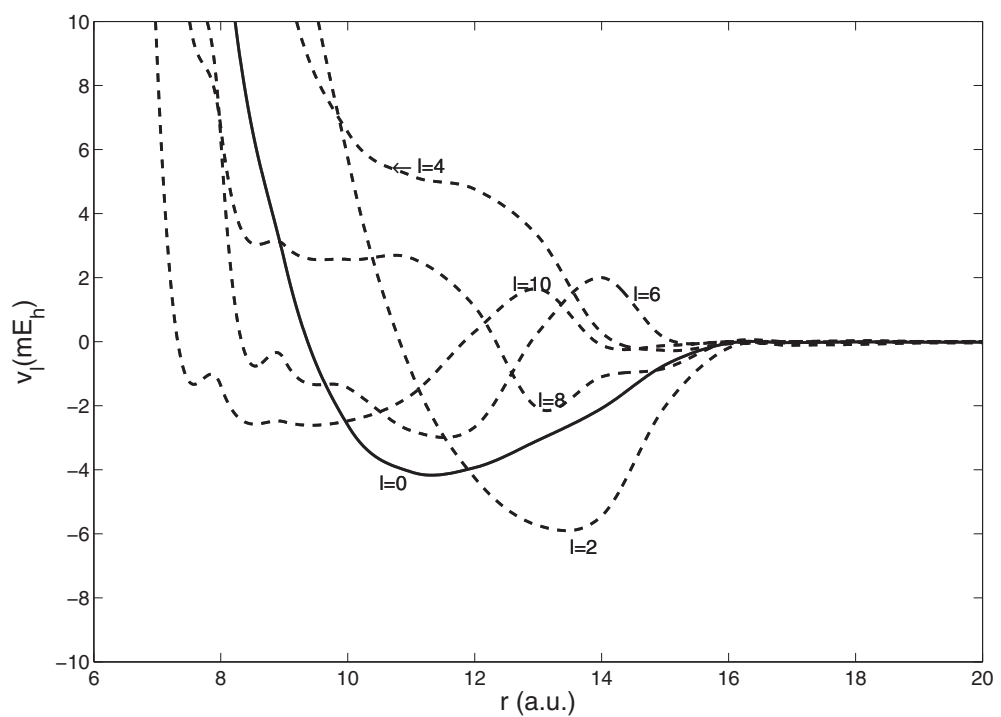

FIG. 13. Different terms $v_{l}(r)$ for $l=0,2,4,6,8,10$ [Eq. (6)] for the $\mathrm{K}_{2}-\mathrm{K}$ interaction. see just how sensitive the scattering cross sections are for changes in the long range potential, we increased the dispersion coefficients (Table III) for the $\mathrm{K}_{2}-\mathrm{K}$ system by $10 \%$ and recalculated the cross sections for $N=0$. The elastic cross section at $T=1 \mathrm{nK}$ increased by about $1000 \%$, whereas the elastic cross section at $T=1 \mathrm{mK}$ decreased by $30 \%$. This certainly demonstrates how sensitive the scattering cross sections are for changes in the long range PES at ultracold temperatures and shows the importance of a reliable long range PES. Similar demonstrations can be made for the $\mathrm{O}_{2}-\mathrm{He}$ and $\mathrm{Na}_{2}-\mathrm{Na}$ systems, although the effect is not as dramatic.

\section{CONCLUSIONS}

We have calculated the PES for three different systems, $\mathrm{O}_{2}-\mathrm{He}, \mathrm{Na}_{2}-\mathrm{Na}$, and $\mathrm{K}_{2}-\mathrm{K}$, with special emphasis on the long range interaction, which as we have seen is of utmost importance for reliable scattering calculations. The van der Waals

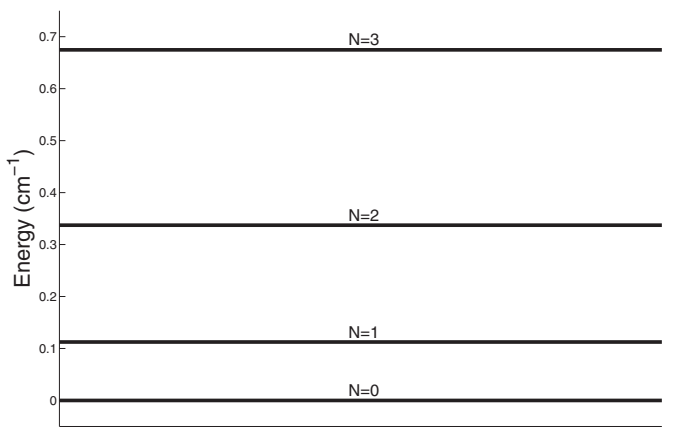

FIG. 14. Rotational energies for the $N=0,1,2,3$ rotational levels in the ground electronic and vibrational state of the $\mathrm{K}_{2}$ molecule. coefficients defining the long range PES have been calculated from the polarizabilities and are expected to be of high accuracy. With the obtained potential energy surfaces we have solved the coupled equations to obtain elastic and inelastic cross sections for transitions between different rotational levels and Zeeman levels (for $\mathrm{O}_{2}-\mathrm{He}$ ).

All reported cross sections have been calculated with a large number of partial waves included, to ensure satisfactory convergence. Most calculations have involved partial waves with $L>20$.

For buffer-gas cooling of $\mathrm{O}_{2}$ molecules with $\mathrm{He}$ atoms we predict macroscopic properties such as speed distributions, temperature, and number of collisions needed to thermalize the $\mathrm{O}_{2}$ molecules based on the calculated cross sections. Magnetic trapping of $\mathrm{O}_{2}$ molecules in the ground electronic and rovibrational state is predicted to be feasible and trap loss from collisions with $\mathrm{He}$ is expected to be limited. Detailed predictions can however not be given as all cross sections are calculated in zero magnetic field.

For all systems the Wigner threshold law is obeyed at low energies and we have found the inelastic cross sections to be larger than the corresponding elastic ones when the energy is sufficiently low, yielding efficient rotational cooling at ultracold temperatures. For $\mathrm{O}_{2}-\mathrm{He}$, the inelastic transitions $N=3$ $\rightarrow 1$ dominates over the corresponding elastic transition for $T<10^{-5} \mathrm{~K}$, whereas the temperatures are respectively $T$ $<10^{-6} \mathrm{~K}$ and $T<10^{-8} \mathrm{~K}$ for the same phenomena to occur in the $\mathrm{Na}_{2}-\mathrm{Na}$ and $\mathrm{K}_{2}-\mathrm{K}$ systems.

Further, the elastic cross sections for the $\mathrm{K}_{2}$ molecule are different by several orders of magnitude depending on the rotational level, favoring the $N=1$ level. This is in contrast to the $\mathrm{Na}_{2}-\mathrm{Na}$ system where the elastic cross section for different rotational levels is of a similar magnitude for all calculated temperatures. Inelastic rotational scattering is for all systems seen to be suppressed for temperatures in the $\mathrm{mK}$ range. 


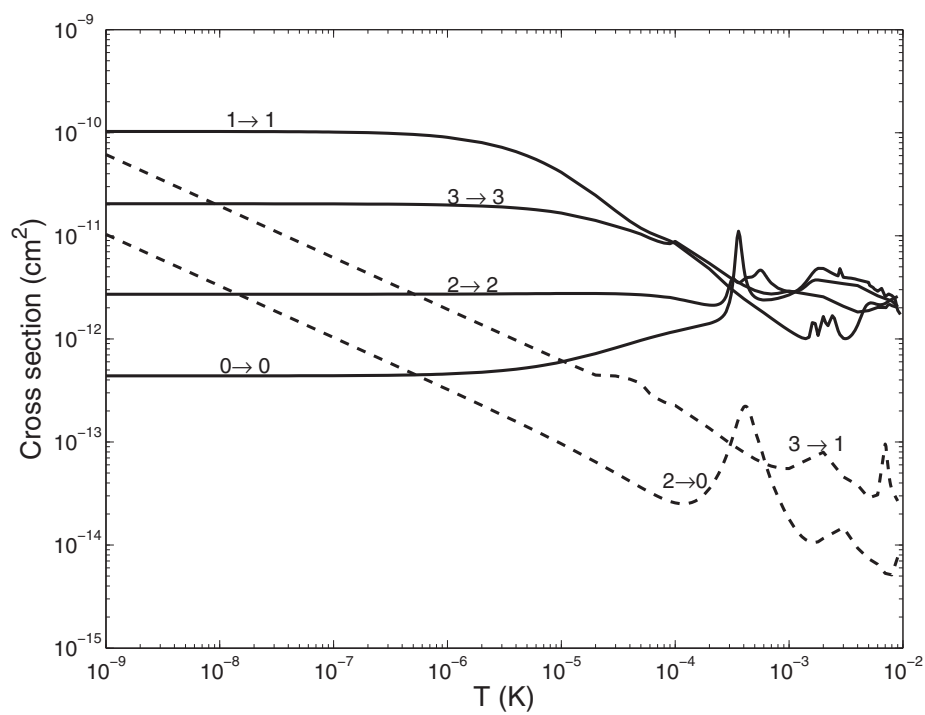

FIG. 15. Elastic and inelastic cross sections for $N=0,1,2,3$ rotational levels in the ground electronic and vibrational state of $\mathrm{K}_{2}$.
For future studies it would be interesting to include magnetic fields and calculate their influence on the cross sections. With relevance to photoassociation experiments it would furthermore be of considerable interest to include excited vibrational levels in a similar study.

\section{ACKNOWLEDGMENT}

Both authors want to acknowledge Inge Røeggen at the University of Troms $\varnothing$ (UiT) for his useful input regarding PES calculations.
[1] J. T. Bahns, W. C. Stwalley, and P. L. Gould, J. Chem. Phys. 104, 9689 (1996).

[2] M. Greiner, C. A. Regal, and D. S. Jin, Nature (London) 426, 537 (2003).

[3] S. Jochim, M. Bartenstein, A. Altmeyer, G. Hendl, S. Riedl, C. Chin, J. H. Denschlag, and R. Grimm, Science 302, 2101 (2003).

[4] M. W. Zwierlein, C. A. Stan, C. H. Schunck, S. M. F. Raupach, S. Gupta, Z. Hadzibabic, and W. Ketterle, Phys. Rev. Lett. 91, 250401 (2003).

[5] H. Feshbach, Ann. Phys. (N.Y.) 5, 357 (1958).

[6] H. R. Thorsheim, J. Weiner, and P. S. Julienne, Phys. Rev. Lett. 58, 2420 (1987).

[7] Y. B. Band and P. S. Julienne, Phys. Rev. A 51, R4317 (1995).

[8] W. C. Stwalley, Prog. Quantum Electron. 8, 203 (1984).

[9] J. M. Doyle, B. Friedrich, J. Kim, and D. Patterson, Phys. Rev. A 52, R2515 (1995).

[10] J. L. Bohn, Phys. Rev. A 62, 032701 (2000).

[11] P. Soldan, M. T. Cvitas, J. M. Hutson, P. Honvault, and J.-M. Launay, Phys. Rev. Lett. 89, 153201 (2002).

[12] M. T. Cvitas, P. Soldan, J. M. Hutson, P. Honvault, and J.-M. Launay, Phys. Rev. Lett. 94, 033201 (2005).

[13] G. Quemener, P. Honvault, J. M. Launay, P. Soldán, D. E. Potter, and J. M. Hutson, Phys. Rev. A 71, 032722 (2005).

[14] A. M. Arthurs and A. Dalgarno, Proc. R. Soc. London, Ser. A
256, 540 (1960).

[15] M. S. Child, Molecular Collision Theory (Academic Press Inc., London, 1974).

[16] D. M. Brink and G. R. Satchler, Angular Momentum, 3rd ed. (Clarendon Press, Oxford, 1993).

[17] B. R. Johnson, J. Comput. Phys. 13, 445 (1973).

[18] M. W. Schmidt et al., J. Comput. Chem. 14, 1347 (1993).

[19] J. H. van Lenthe and F. B. van Duijneveldt, J. Chem. Phys. 81, 3168 (1984).

[20] C. M. Aikens, S. P. Webb, R. L. Bell, G. D. Fletcher, M. Schmidt, and M. S. Gordon, Theor. Chem. Acc. 110, 233 (2003).

[21] T. H. Dunning, J. Chem. Phys. 90, 1007 (1989).

[22] D. E. Woon and T. H. Dunning, Jr., J. Chem. Phys. 103, 4572 (1995).

[23] D. E. Woon and T. H. Dunning, Jr., J. Chem. Phys. 100, 2975 (1994).

[24] A. D. McLean and G. S. Chandler, J. Chem. Phys. 72, 5639 (1980).

[25] G. C. M. Gutowski, F. B. van Duijneveldt, G. Chałasiński and L. Piela, Mol. Phys. 61, 233 (1986).

[26] S. F. Boys and F. Bernardi, Mol. Phys. 19, 553 (1970).

[27] L. Veseth, Phys. Rev. A 44, 358 (1991).

[28] D. M. Bishop and J. Pipin, Int. J. Quantum Chem. 45, 349 (1993).

[29] U. Hohm, Chem. Phys. 179, 533 (1994). 
[30] L. Veseth and A. Lofthus, Mol. Phys. 27, 511 (1974).

[31] B. H. Bransden and C. J. Joachain, Physics of Atoms and Molecules (Longman Scientific \& Technical, New York, 1983).
[32] E. P. Wigner, Phys. Rev. 73, 1002 (1948).

[33] G. A. Bird, Molecular Gas Dynamics (Clarendon Press, Oxford, 1976).

[34] K. Koura, Phys. Fluids 29, 3509 (1986). 


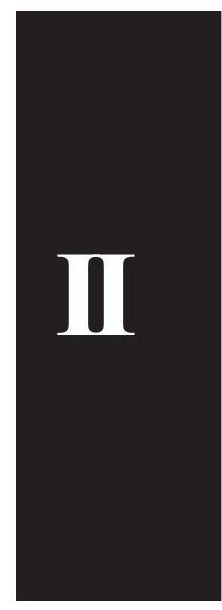





\title{
$A b$ initio calculation of Feshbach resonances in cold atomic collisions: $s$ - and $p$-wave Feshbach resonances in ${ }^{6} \mathrm{Li}_{2}$
}

\author{
M. Lysebo* and L. Veseth \\ Department of Physics, University of Oslo, 0316 Oslo, Norway
}

(Received 30 March 2009; published 5 June 2009)

\begin{abstract}
We present a model applicable to cold diatomic collisions and solve the close-coupled equations for the particular ${ }^{6} \mathrm{Li}_{2}$ system. Feshbach resonances are determined $a b$ initio for both $s$ and $p$ waves. The $s$-wave scattering lengths are reported for all possible hyperfine states as a function of magnetic field strengths between 0 and $1500 \mathrm{G}$. In addition, $p$-wave scattering lengths are calculated for selected hyperfine states. Matrix elements for the hyperfine, Zeeman, and rotational Hamiltonian were worked out by the use of the molecular Hund's case (a) basis set. All relevant matrix elements are reported. The short-range hyperfine interaction has been included in the calculations, and its effect on the scattering length are investigated. Hyperfine parameters were obtained from separate $a b$ initio calculations.
\end{abstract}

DOI: 10.1103/PhysRevA.79.062704

PACS number(s): $34.50 . \mathrm{Cx}, 32.10 . \mathrm{Fn}$

\section{INTRODUCTION}

Feshbach resonances [1] named after Herman Feshbach have become very important in ultracold atomic and molecular physics for the last ten years. Feshbach resonances are important ingredients in the ongoing exploration of these systems and in the tremendous progress made after the first successful creation of a Fermi gas [2]. In ultracold atomic and molecular physics, the important and relevant Feshbach resonances occur when the energy of a bound molecular state coincides with that of two colliding atoms. The coupling between the bound molecular state and the continuum of states is caused by the hyperfine interaction in homonuclear molecules and by the hyperfine interaction together with electronic spin-dependent interactions in heteronuclear molecules. Thus, the hyperfine interaction plays an important role. The experimental and theoretical focus this far has been on alkali atoms.

The attractive properties of these systems stem from the possibility of tuning the interatomic interaction by varying the magnetic field around a Feshbach resonance. The scattering length is the important parameter which characterizes the atom-atom interaction in ultracold gases [3] (together with the Pauli principle). The scattering length behaves in a peculiar way as one passes through a Feshbach resonance-it diverges and changes sign (see, e.g., Fig. 6). This makes it possible for the experimentalists to attain full control of the interactions by varying the magnetic field strength. Many systems have Feshbach resonances that are accessible through relatively low magnetic field strengths.

The Feshbach resonances also offer the opportunity to produce weakly bound ultracold molecules [4]. Using a sequence of magnetic pulses, the ultracold atoms can be converted into molecules with lifetimes of several seconds [5-9]. Initially, one thought that such highly excited molecules would decay very fast. This is true for bosonic atoms, where inelastic collisions with other atoms or molecules give a rapid decay. However, for fermionic atoms, the molecules

\footnotetext{
*marius.lysebo@fys.uio.no
}

have a much longer lifetime due to quenching of the inelastic collision rates as a result of the Pauli principle. In part, this is why the fermionic isotope ${ }^{6} \mathrm{Li}$ are preferred over the bosonic isotope ${ }^{7} \mathrm{Li}$, which is far less studied. The first molecular Bose-Einstein condensates (BECs) observed in November 2003 utilized molecules created from Feshbach resonances. Jochim et al. [10] used ${ }^{40} \mathrm{~K}$ atoms, while Greiner et al. [11] and Bartenstein et al. [12] worked with ${ }^{6} \mathrm{Li}$ atoms. The $\mathrm{nK}$ temperature range and BEC were eventually reached by evaporative cooling.

Finally, the ability to tune the scattering length made the so-called BEC-BCS transition experimentally accessible $[13,14]$. In short, this is a transition from a molecular BEC to atomic Cooper pairs via a regime where the scattering length diverges and becomes infinite. In the crossover regime, the interaction is independent of the scattering length as well as the range and details of the interatomic potential [15].

This paper focuses on cold diatomic collisions in general, and, in particular, the ${ }^{6} \mathrm{Li}_{2}$ system and on the Feshbach resonances in this system. Most attention is given to the wave Feshbach resonances, but we also calculate Feshbach resonances occurring in $p$ waves. Rather than to set up a model with one or more tunable parameters, our philosophy has been to perform $a b$ initio calculations of the various resonances using a fully coupled-channel approach. No empirical data have been included in the calculations. The calculations are performed with the diatomic Hund's case (a) basis set, describing the system as a molecule rather than two free atoms. This allows for a realistic and more accurate description of the short-range hyperfine interaction through the calculation of only a few molecular hyperfine parameters. Most, if not all, studies of diatomic collisions make no distinction between the short-range and the long-range hyperfine interaction, i.e., the hyperfine interaction is assumed to be independent of the internuclear separation. Our approach to the hyperfine interaction enables us to test this approximation. However, in the present case we find that the long-range atomic hyperfine interaction makes a reasonable approximation to the short-range case. This fact is due to the very shallow character of the relevant molecular state. In the next sections, we give a thorough explanation of the particular 
model that we use and relate the Hund's case (a) basis set to the more standard atomic $F F$-coupled basis sets.

The model and the expressions we present in this paper are not fully general. Some simplifications have been possible due to the particular alkali system studied. However, we do make it clear whenever special assumptions have been made. Throughout this paper, atomic units $\hbar=m_{e}=e=a_{0}=1$ are used, and we work within the Born-Oppenheimer approximation.

\section{THEORY}

The diatomic Hamiltonian can be written as

$$
H=-\frac{1}{2 \mu} \nabla^{2}+H_{\mathrm{ev}}(\mathbf{r}, \mathbf{x})+H_{\mathrm{hf}}(\mathbf{r}, \mathbf{x})+H_{\mathrm{Z}}(\mathbf{r}, \mathbf{x}),
$$

where $\mathbf{r}$ is the internuclear vector pointing from nuclei $A$ to nuclei $B$ and $\mathbf{x}=\left[\mathbf{x}_{1}, \mathbf{x}_{2}, \ldots, \mathbf{x}_{n}\right]^{T}$ contains the electronic coordinates. $H_{\mathrm{ev}}$ is the electronic and vibrational Hamiltonian, $H_{\mathrm{hf}}$ is the hyperfine Hamiltonian, and finally $H_{\mathrm{Z}}$ denotes the Zeeman interaction. $H_{\mathrm{ev}}$ can also include all types of relativistic contributions such as spin-orbit and spin-spin interactions if needed. $\mu$ is the reduced mass. The first task is to expand the total wave function in a chosen basis, e.g., the eigenstates of $H_{\mathrm{ev}}$ or some other operator. At short range, the eigenstates of $H_{\mathrm{ev}}$ are well suited; whereas at long range the eigenstates of $H_{\mathrm{hf}}+H_{\mathrm{Z}}$ diagonalizes the interaction. To solve the time-independent Schrödinger equation for the whole molecule

$$
H \Psi(\mathbf{r}, \mathbf{x})=E \Psi(\mathbf{r}, \mathbf{x}),
$$

we write

$$
\Psi(\mathbf{r}, \mathbf{x})=\sum_{b} \frac{1}{r} \psi_{b}(r) \phi_{b}(\mathbf{r}, \mathbf{x}),
$$

where $\psi_{b}(r)$ is the radial-wave functions for the relative motion of the two nuclei for a molecule in electronic state $b$. The angular part of the relative motion is included in $\phi_{b}(\mathbf{r}, \mathbf{x})$. Thus, we may write the time-independent Schrödinger equation (2) as

$$
\begin{aligned}
& -\frac{1}{2 \mu} \nabla^{2} \sum_{b} \frac{1}{r} \psi_{b}(r) \phi_{b}(\mathbf{r}, \mathbf{x})+\left(H_{\mathrm{ev}}+H_{\mathrm{hf}}+H_{\mathrm{Z}}\right) \\
& \quad \times \sum_{b} \frac{\psi_{b}(r)}{r} \phi_{b}(\mathbf{r}, \mathbf{x}) \\
& =E \sum_{b} \frac{\psi_{b}(r)}{r} \phi_{b}(\mathbf{r}, \mathbf{x}) .
\end{aligned}
$$

Multiplying with $\phi_{a}^{*}(\mathbf{r}, \mathbf{x})$ and integrating over the electronic coordinates yields

$$
\begin{aligned}
& -\frac{1}{2 \mu} \frac{d^{2} \psi_{a}(r)}{d r^{2}}+\frac{l_{a}\left(l_{a}+1\right)}{2 \mu r^{2}} \psi_{a}(r)+\sum_{b}\left[V_{a b}^{\mathrm{ev}}+V_{a b}^{\mathrm{hf}}+V_{a b}^{\mathrm{Z}}\right] \psi_{b}(r) \\
& \quad=E \psi_{a}(r)
\end{aligned}
$$

where

$$
\begin{gathered}
V_{a b}^{\mathrm{ev}}(r)=\left\langle\phi_{a}(\mathbf{r}, \mathbf{x})\left|H_{\mathrm{ev}}\right| \phi_{b}(\mathbf{r}, \mathbf{x})\right\rangle, \\
V_{a b}^{\mathrm{hf}}(r)=\left\langle\phi_{a}(\mathbf{r}, \mathbf{x})\left|H_{\mathrm{hf}}\right| \phi_{b}(\mathbf{r}, \mathbf{x})\right\rangle, \\
V_{a b}^{\mathrm{Z}}=\left\langle\phi_{a}(\mathbf{r}, \mathbf{x})\left|H_{\mathrm{Z}}\right| \phi_{b}(\mathbf{r}, \mathbf{x})\right\rangle,
\end{gathered}
$$

and $l_{a}$ is the quantum number associated with the angular momentum of the relative motion of the two atoms. Deriving Eq. (5) from Eq. (4), it has been assumed that the nonadiabatic coupling terms can be ignored, and we make the approximation [16]

$$
\sum_{b} \int \phi_{a}(\mathbf{r}, \mathbf{x})^{*} \nabla^{2} \phi_{b}(\mathbf{r}, \mathbf{x}) \frac{\psi_{b}(r)}{r} d \mathbf{x}=\frac{d^{2} \psi_{a}}{d r^{2}}+\frac{l_{a}\left(l_{a}+1\right)}{r^{2}} \psi_{a}(r) .
$$

Finally, we have the boundary conditions $\psi_{a}(0)=0$ and

$$
\psi_{a}(r) \underset{r \rightarrow \infty}{\rightarrow} \sum_{b} \phi_{b}(\mathbf{r}, \mathbf{x}) \frac{1}{\sqrt{k_{b}}}\left[j_{b}\left(k_{b} r\right) \delta_{a b}+g_{b}\left(k_{b} r\right) K_{b a}\right],
$$

where $K$ is the so-called reaction matrix determined from Eq. (10). $j_{l}(x)$ and $g_{l}(x)$ are the Riccati-Bessel functions [17] for real $k_{b}=\sqrt{2 \mu\left(E-E_{b}\right)}$ (i.e., open channels $E>E_{b}$ ) but must be replaced with the modified spherical Bessel functions [17] of first and third kinds multiplied with $k_{b} \sqrt{r}$ for all closed channels. The important $S$ matrix can be determined once $K$ is known [18],

$$
S=\frac{I+i K_{00}}{I-i K_{00}}
$$

where $K_{00}$ is a submatrix of $K$ containing all the elements connecting open channels ( $I$ is the identity matrix). The $S$-matrix elements readily yield the important phase shift $\delta_{l}$ from the simple expression

$$
S_{a a}=e^{2 i \delta_{l}},
$$

where $l$ is the partial-wave quantum number.

\section{A. Choice of basis set}

To simplify the equations presented in this section, the two colliding atoms have been assumed to be in $S$ states. For atoms in other states, e.g., $P$ states, a nonzero angular momentum must be included. See the Appendix for more details.

\section{Long-range basis: FF-coupled states}

The long-range electronic potential energy $V(r)$ for two atoms, where $L_{i}=0$ is proportional to $r^{-6}$ [19]. Asymptotically, as $r \rightarrow \infty$, we are then left with the hyperfine Hamiltonian together with the Zeeman interaction set up by an external magnetic field. To fulfill the boundary condition imposed as $r \rightarrow \infty$, we choose a basis in which these interactions are diagonal. Consider the situation where atom 1 has total angular momentum $\mathbf{F}_{1}$ and atom 2 has total angular momentum $\mathbf{F}_{2}$. The atomic hyperfine interaction is diagonal in this basis if $\mathbf{J}_{1} \equiv \mathbf{L}_{1}+\mathbf{S}_{1}, \mathbf{J}_{2} \equiv \mathbf{L}_{2}+\mathbf{S}_{2}, \mathbf{I}_{1}$ and $\mathbf{I}_{2}$ are good quantum 
numbers together with $M_{F_{1}}$ and $M_{F_{2}}$. The total two-atom angular-momentum states are constructed by adding the single atom angular momentum $\mathbf{F}_{i}$ from both atoms together with the mechanical rotation of the relative motion $\mathbf{L}$ (not to be confused with $\mathbf{L}_{1}$ or $\mathbf{L}_{2}$ ) to give the total angular momentum $\mathbf{F}$. First, we define the total atomic angular momentum $\mathbf{F}_{a}=\mathbf{F}_{1}+\mathbf{F}_{2}$,

$$
\begin{aligned}
\left|F_{1} F_{2} F_{a} M_{F_{a}}\right\rangle= & \sum_{M_{F_{1}}, M_{F_{2}}}\left\langle F_{1} M_{F_{1}} F_{2} M_{F_{2}} \mid F_{a} M_{F_{a}}\right\rangle\left|F_{1} M_{F_{1}}\right\rangle \\
& \times\left|F_{2} M_{F_{2}}\right\rangle .
\end{aligned}
$$

Finally, we include also the angular momentum $\mathbf{L}$ from the relative motion of the two collision partners and construct the total angular momentum $\mathbf{F}=\mathbf{F}_{a}+\mathbf{L}$. The quantum numbers $l$ and $m_{l}$ are introduced as eigenvalues of the operators $\mathbf{L}^{2}$ and $L_{z}$, respectively, with the spherical harmonics $\left\langle\mathbf{r} \mid l m_{l}\right\rangle=Y_{l m_{l}}(\theta, \phi)$ as their eigenfunctions,

$$
\left|F_{a} l F M_{F}\right\rangle=\sum_{M_{F_{a}}, m_{l}}\left\langle F_{a} M_{F_{a}} l m_{l} \mid F M_{F}\right\rangle\left|F_{a} M_{F_{a}}\right\rangle\left|l m_{l}\right\rangle .
$$

We will refer to this basis as the $F F$-coupled basis.

Whenever an external magnetic field $B$ is introduced, the Zeeman interaction couples the magnetic field to the electronic spins and $\left|F_{a} l F M_{F}\right\rangle$ are no longer eigenstates of the long-range interaction. The long-range interaction is the sum $H_{\mathrm{hf}}+H_{\mathrm{Z}}$ which are diagonalized numerically. The remaining good quantum numbers in an external magnetic field are the total projection $M_{F}=M_{F_{1}}+M_{F_{2}}$ on a space-fixed axis and the mechanical rotation quantum number $l$.

\section{Short-range basis: Molecular Hund's case (a) states}

Here we define the molecular Hund's case (a) states. The matrix elements of the different interactions will be represented in this basis. For small distances $r$, the intuitive choice is a basis which describes the system as a molecule rather than two atoms. One possible choice is then to quantize $\mathbf{S}^{2}$ $=\left(\mathbf{S}_{1}+\mathbf{S}_{2}\right)^{2}$ together with $S_{z}$ and $L_{\mathrm{z}}$ with quantum numbers $S, \Sigma$, and $\Lambda$. The quantum numbers $\Sigma$ and $\Lambda$ are projections on the interatomic axis in a molecule-fixed system. We also need the nuclear-spin states $\left|I_{1} \Omega_{I_{1}}\right\rangle$ and $\left|I_{2} \Omega_{I_{2}}\right\rangle$. Similarly, $\Omega_{I_{1}}$ and $\Omega_{I_{2}}$ are projections of the nuclear spin on the internuclear axis in the molecule-fixed system. Combined to give the total angular momentum $\mathbf{F}$ (see the Appendix), we obtain the basis states

$\left|q \Lambda S \Sigma \Omega_{I_{1}} \Omega_{I_{2}} F \Omega_{F} M_{F}\right\rangle=|q \Lambda S \Sigma\rangle\left|\Omega_{I_{1}} \Omega_{I_{2}}\right\rangle\left|F \Omega_{F} M_{F}\right\rangle$,

where $\Omega_{F}=\Sigma+\Lambda+\Omega_{I_{1}}+\Omega_{I_{2}}$ is the projection of the total spin on the internuclear axis. These are the Hund's case (a) basis states. The letter $q$ in the state symbol represents the other quantum numbers necessary to specify the electronic state. The quantum numbers $I_{1}$ and $I_{2}$ are constants and have been suppressed in the notation.

\section{Unitary transformation between the short-range basis and the long-range basis}

Although we will be mostly concerned with the molecular Hund's case (a) states, we obtain the useful unitary transformation between the $F F$-coupled states, where the $K$ matrix is defined [see Eq. (10)], and the Hund's case (a) states. The unitary transformation has been worked out in detail in the Appendix; here we merely give the final expression in terms of $3 \mathrm{j}$-symbols,

$$
\begin{aligned}
& \left\langle q S \Sigma \Omega_{I_{1}} \Omega_{I_{2}} F M_{F} \Omega_{F} \mid q F_{1} F_{2} F_{a} l F M_{F}\right\rangle \\
& =(-1)^{f} \sum_{\substack{M_{F_{a}}, m_{l} \\
M_{F_{1}}, M_{F_{2}}}} \sum_{\substack{M_{S_{1}}, M_{S_{2}}, M_{I_{2}}, M_{S}, M_{S} \\
M_{S}}}(2 J+1)(2 I+1)(2 F+1)\left[F_{a}\right]\left[F_{1}\right]\left[F_{2}\right][S][l]\left(\begin{array}{ccc}
l & F_{a} & F \\
m_{l} & M_{F_{a}} & -M_{F}
\end{array}\right)\left(\begin{array}{ccc}
F_{1} & F_{2} & F_{a} \\
M_{F_{1}} & M_{F_{2}} & -M_{F_{a}}
\end{array}\right) \\
& \times\left(\begin{array}{ccc}
S_{1} & I_{1} & F_{1} \\
M_{S_{1}} & M_{I_{1}} & -M_{F_{1}}
\end{array}\right)\left(\begin{array}{ccc}
S_{2} & I_{2} & F_{2} \\
M_{S_{2}} & M_{I_{2}} & -M_{F_{2}}
\end{array}\right)\left(\begin{array}{ccc}
S_{1} & S_{2} & S \\
M_{S_{1}} & M_{S_{2}} & -M_{S}
\end{array}\right)\left(\begin{array}{ccc}
S & l & J \\
M_{S} & m_{l} & -M_{J}
\end{array}\right)\left(\begin{array}{ccc}
S & l & J \\
\Sigma & 0 & -\Omega
\end{array}\right) \\
& \times\left(\begin{array}{ccc}
I_{1} & I_{2} & I \\
M_{I_{1}} & M_{I_{2}} & -M_{I}
\end{array}\right)\left(\begin{array}{ccc}
I_{1} & I_{2} & I \\
\Omega_{I_{1}} & \Omega_{I_{2}} & -\Omega_{I}
\end{array}\right)\left(\begin{array}{ccc}
J & I & F \\
M_{J} & M_{I} & -M_{F}
\end{array}\right)\left(\begin{array}{ccc}
J & I & F \\
\Omega & \Omega_{I} & -\Omega_{F}
\end{array}\right),
\end{aligned}
$$

with $f=F_{a}-l+F_{1}-F_{2}+M_{F_{a}}+2 S_{1}-I_{1}+M_{F_{1}}-I_{2}+M_{F_{2}}+M_{S}$ $+\Omega+\Omega_{I}+\Omega_{F}-M_{J}-M_{I},\left[F_{a}\right]=\sqrt[3]{2 F_{a}+1}$, and similar for other quantities in square brackets. When $L_{i} \neq 0$, the unitary transformation (16) is no longer valid.

The unitary transformation from the case (a) basis to the $F F$-coupled basis is often called a frame transformation in the present context (see [20,21]). For further discussion of other convenient basis states and a more in-depth treatment, we refer to $[16,22]$ and references therein.

\section{B. Pauli exclusion principle}

Due to the Pauli principle, the wave function describing our system must have certain symmetries upon the interchange of identical particles. Working with two identical at- 
oms, the proper wave function must fulfill two requirements. (1) Interchanging an even (odd) number of electrons should yield a phase factor $+1(-1)$, whereas (2) interchanging the nuclei should give +1 or -1 depending on whether $I_{1}=I_{2}$ is integer or half integer (boson or fermion, respectively). The long-range and short-range states introduced in Sec. II A must respect these symmetries. The following discussion is, in particular, relevant for two alkali atoms with identical nuclei $L_{1}=L_{2}=0$ and $S_{1}=S_{2} \neq 0$. However, for $L_{1}=L_{2} \neq 0$, the modifications to the expressions given are rather straightforward.

In addition to the previously mentioned Hund's case (a) basis, we introduce the Hund's case (b) basis as the symmetries are easier to deduce in this basis. This is due to the fact that both the electronic and the nuclear spin is quantized in a space-fixed system $\left(\Sigma \rightarrow M_{S}, \Omega_{I_{1}} \rightarrow M_{I_{1}}\right.$, and $\left.\Omega_{I_{2}} \rightarrow M_{I_{2}}\right)$ in a case (b) basis. See [23] for a further discussion of the different Hund's cases.

Both basis set $|q \Lambda S \Sigma\rangle$ [case (a)] and $\left|q \Lambda S M_{S}\right\rangle$ [case (b)] can be constructed as eigenstates for the total inversion operator $I_{t}$ (spatial inversion in a space-fixed system) for all diatomic molecules. We denote the eigenvalues of $I_{t}$ with \pm . Assuming $M_{L_{1}}=M_{L_{2}}=0$, it can be shown that the only possible eigenvalue for $I_{t}$ is +1 [24]. For homonuclear diatomic molecules, there are two additional symmetry operations. These are inversion of the spatial nuclear coordinates $I_{n}$ and inversion of the spatial electronic coordinates $I_{e}$ with eigenvalue $(-1)^{i_{e}}$. For all operators, inversion is performed in a space-fixed coordinate system. Electronic states with $i_{e}=1$ are denoted $u$ (ungerade), whereas states with $i_{e}=0$ are $g$ states (gerade). These three symmetry operations are also related through the relation $I_{t}=I_{n} I_{e}$. We will assume that we are working with states $|q \Lambda S \Sigma\rangle$ and $\left|q \Lambda S M_{S}\right\rangle$ that are eigenstates for the symmetry operators $I_{t}, I_{e}$ and $I_{n}$.

The electronic case (b) states $\left|q \Lambda S M_{S}\right\rangle$ are linear combinations of spin orbitals $q_{\Lambda}(\mathbf{x}, r)\left|S M_{S}\right\rangle$. To determine their behavior under symmetry operations, it suffices to consider $q_{\Lambda}(\mathbf{x}, r)$ and $\left|S M_{S}\right\rangle$ separately [25,26]. However, it is important to realize that the separation $\left|q \Lambda S M_{S}\right\rangle=|q \Lambda\rangle\left|S M_{S}\right\rangle$ is only generally valid for one- and two-electron molecules.

Next we introduce the electronic permutation operator $P_{e}$ that permutes all the electrons. Both the spatial and the spin part of $\left|q \Lambda S M_{S}\right\rangle$ are eigenstates of $P_{e}$. The eigenvalues corresponding to the spatial functions $q_{\Lambda}(\mathbf{x}, r)$ are $(-1)^{p_{e}}$ where $p_{e}=0$ (symmetric) and $p_{e}=1$ (antisymmetric). There exists an intimate but not obvious connection between the eigenvalues of the operators $I_{e}$ and $P_{e}$ when acting on $q_{\Lambda}(\mathbf{x}, r)$. It can be shown [26] that the eigenvalues are related through the relation $(-1)^{i_{e}}= \pm(-1)^{p_{e}}$, where \pm are the eigenvalues of the $I_{t}$ operator, restricted to +1 for the present system. It then follows that $(-1)^{i_{e}}=(-1)^{p_{e}}$, hence $P_{e}$ and $I_{e}$ have identical eigenvalues acting on $q_{\Lambda}(\mathbf{x}, r)$.

The result of the operation $P_{e}\left|S M_{S}\right\rangle$ is easily worked out due to the symmetry of the Clebsch-Gordan coefficients, and one obtains $P_{e}\left|S M_{S}\right\rangle=(-1)^{2 S_{1}-S}\left|S M_{S}\right\rangle$. To summarize, we may then write

$$
P_{e}\left|q \Lambda S M_{S}\right\rangle=(-1)^{i_{e}+2 S_{1}-S}\left|q \Lambda S M_{S}\right\rangle .
$$

However, the Pauli principle requires

$$
P_{e}\left|q \Lambda S M_{S}\right\rangle=(-1)^{N_{1}}\left|q \Lambda S M_{S}\right\rangle
$$

where $N_{1}$ is the number of electron permutations. Combined with Eq. (17), this implies $(-1)^{i_{e}+2 S_{1}-S}=(-1)^{N_{1}}$. Since $(-1)^{2 S_{1}}=(-1)^{N_{1}}$, it follows that only electronic states where $(-1)^{i_{e}-S}=+1$ are allowed. These are the ${ }^{1} \Sigma_{g}^{+}$and ${ }^{3} \Sigma_{u}^{+}$states found in diatomic alkali molecules.

In general, the allowed combinations of $g / u$ and electronic spin $S$ can be worked out from the Wigner-Witmer rules [27]. See also [26] and references therein for a more accessible derivation of the Wigner-Witmer rules.

We have found that $I_{t}\left|q \Lambda S M_{S}\right\rangle=+1\left|q \Lambda S M_{S}\right\rangle$ and that $I_{e}\left|q \Lambda S M_{S}\right\rangle=(-1)^{i_{e}}\left|q \Lambda S M_{S}\right\rangle\left(I_{t}, I_{e}\right.$ and $I_{n}$ only act on the spatial coordinates, leaving the spin states $\left|S M_{S}\right\rangle$ unaffected). From the relation $I_{n}=I_{t} I_{e}$, it then follows that $I_{n}\left|q \Lambda S M_{S}\right\rangle$ $=(-1)^{i_{e}}\left|q \Lambda S M_{S}\right\rangle$. However, inversion and permutation of two nuclei (in a diatomic molecule) in space-fixed axis with origin at the center-of-mass position are the same two operations. Thus we conclude

$$
I_{n}\left|q \Lambda S M_{S}\right\rangle=P_{n}\left|q \Lambda S M_{S}\right\rangle=(-1)^{i}\left|q \Lambda S M_{S}\right\rangle .
$$

However, it is not obvious how the electronic case (a) states defined in molecule-fixed axes are affected by $P_{n}$. To determine this, we use the relation between case (a) and case (b) states

$$
\left|q \Lambda S M_{S}\right\rangle=\sum_{\Sigma} \mathcal{D}_{M_{S}, \Sigma}^{S}(\phi, \theta, 0)^{*}|q \Lambda S \Sigma\rangle,
$$

where $\mathcal{D}$ is the rotation matrix [28] that rotates the spacefixed component of $M_{S}$ into the molecule-fixed $\Sigma$ component. The effect of $P_{n}$ is the same as a rotation of the axis of the molecule by an angle $\pi$, which is equivalent to the transformation $[(\theta, \phi) \rightarrow(\pi-\theta, \phi+\pi)]$. The property

$$
\begin{aligned}
P_{n} \mathcal{D}_{M_{S}, \Sigma}^{S}(\phi, \theta, 0)^{*} & =\mathcal{D}_{M_{S}, \Sigma}^{S}(\phi+\pi, \pi-\theta, 0)^{*} \\
& =(-1)^{S} \mathcal{D}_{M_{S},-\Sigma}^{S}(\phi, \theta, 0)^{*}
\end{aligned}
$$

which follows from the definition of the $\mathcal{D}$ matrix elements is then very useful. Knowing how $P_{n}$ acts on both $\left|q \Lambda S M_{S}\right\rangle$ and $\mathcal{D}_{M_{S} \Sigma}^{S}(\phi, \theta, 0)^{*}$, it is seen from Eq. (20) that

$$
P_{n}|q \Lambda S \Sigma\rangle=(-1)^{-S+i_{e}}|q \Lambda S-\Sigma\rangle,
$$

in agreement with [29]. For a more detailed treatment, see Zare et al. [25] and references therein.

We also need to consider the nuclear-spin states $\left|I_{1} M_{I_{1}}\right\rangle\left|I_{2} M_{I_{2}}\right\rangle=\left|I_{1} M_{I_{1}} I_{2} M_{I_{2}}\right\rangle$ under permutation of the nuclei $P_{n}$. Working with the case (b) basis in space-fixed axis, it follows immediately that $P_{n}\left|I_{1} M_{I_{1}} I_{2} M_{I_{2}}\right\rangle=\left|I_{2} M_{I_{2}} I_{1} M_{I_{1}}\right\rangle$. However, to obtain $P_{n}\left|I_{1} \Omega_{I_{1}} I_{2} \Omega_{I_{2}}\right\rangle$ we write

$$
\left|I_{1} M_{I_{1}} I_{2} M_{I_{2}}\right\rangle=\sum_{\Omega_{I_{1}}} \mathcal{D}_{M_{I_{1}}, \Omega_{I_{1}}}^{I_{1}}(\phi, \theta, 0)^{*}\left|I_{1} \Omega_{I_{1}} I_{2} M_{I_{2}}\right\rangle,
$$

or alternatively 


$$
\begin{aligned}
& \left|I_{2} M_{I_{2}} I_{1} M_{I_{1}}\right\rangle=\sum_{\Omega_{I_{1}}} \mathcal{D}_{M_{I_{1}}, \Omega_{I_{1}}}^{I_{1}}(\phi, \theta, 0)^{*}\left|I_{2} M_{I_{2}} I_{1} \Omega_{I_{1}}\right\rangle \\
& \quad=P_{n}\left[\sum_{\Omega_{I_{1}}} \mathcal{D}_{M_{I_{1}}, \Omega_{I_{1}}}^{I_{1}}(\phi, \theta, 0)^{*}\left|I_{1} \Omega_{I_{1}} I_{2} M_{I_{2}}\right\rangle\right] \\
& =(-1)^{I_{1}} \sum_{\Omega_{I_{1}}} \mathcal{D}_{M_{I_{1}}, \Omega_{I_{1}}}^{I_{1}}(\phi, \theta, 0)^{*} P_{n}\left|I_{1} \Omega_{I_{1}} I_{2} M_{I_{2}}\right\rangle,
\end{aligned}
$$

hence $P_{n}\left|I_{1} \Omega_{I_{1}} I_{2} M_{I_{2}}\right\rangle=(-1)^{-I_{1}}\left|I_{2} M_{I_{2}} I_{1}-\Omega_{I_{1}}\right\rangle$. Similarly, rotating $M_{I_{2}}$ into $\Omega_{I_{2}}$ yields

$$
P_{n}\left|I_{1} \Omega_{I_{1}} I_{2} \Omega_{I_{2}}\right\rangle=(-1)^{-2 I_{1}}\left|I_{2}-\Omega_{I_{2}} I_{1}-\Omega_{I_{1}}\right\rangle .
$$

Finally, we determine the effect of $P_{n}$ on the total angularmomentum states $\left|F M_{F} \Omega_{F}\right\rangle .\left\langle\mathbf{r} \mid F M_{F} \Omega_{F}\right\rangle$ are given in terms of the $\mathcal{D}_{M_{F} \Omega_{F}}(\phi, \theta, 0)^{*}$ rotation matrix elements [30],

$$
\Psi_{F M_{F} \Omega_{F}}(\phi, \theta)=\left\langle\mathbf{r} \mid F M_{F} \Omega_{F}\right\rangle=\sqrt{\frac{2 F+1}{4 \pi}} \mathcal{D}_{M_{F} \Omega_{F}}^{F}(\phi, \theta, 0)^{*},
$$

and the phase factor is easily determined from Eq. (21) with $F$ in place of $S$, and with similar replacements for $M_{F}$ and $\Omega_{F}$. The result is

$$
P_{n}\left|F M_{F} \Omega_{F}\right\rangle=(-1)^{F}\left|F M_{F}-\Omega_{F}\right\rangle .
$$

Combining Eqs. (22), (25), and (27), we obtain

$$
\begin{aligned}
& P_{n}\left|q \Lambda S \Sigma \Omega_{I_{1}} \Omega_{I_{2}} F M_{F} \Omega_{F}\right\rangle \\
& \quad=(-1)^{-S+i_{e}-2 I_{1}+F}\left|q \Lambda S-\Sigma-\Omega_{I_{1}}-\Omega_{I_{2}} F M_{F}-\Omega_{F}\right\rangle,
\end{aligned}
$$

and case (a) states that are eigenstates for the $P_{n}$ operator and, fulfill, the Pauli principle can be constructed,

$$
\begin{aligned}
\left|q \Lambda S \Sigma \Omega_{I_{1}} \Omega_{I_{2}} F M_{F} \Omega_{F}\right\rangle= & \frac{1}{\sqrt{2}}\left(\left|q \Lambda S \Sigma \Omega_{I_{1}} \Omega_{I_{2}} F M_{F} \Omega_{F}\right\rangle\right. \\
& +(-1)^{-S+i_{e}+F} \mid q-\Lambda S-\Sigma-\Omega_{I_{2}} \\
& \left.-\Omega_{I_{2}} F M_{F}-\Omega_{F}\right) .
\end{aligned}
$$

The Pauli principle requires the states $\left|q \Lambda S \Sigma \Omega_{I_{1}} \Omega_{I_{2}} F M_{F} \Omega_{F}\right\rangle$ to have the following symmetry:

$$
P_{n}\left|q \Lambda S \Sigma \Omega_{I_{1}} \Omega_{I_{2}} F M_{F} \Omega_{F}\right\rangle=(-1)^{2 I_{1}}\left|q \Lambda S \Sigma \Omega_{I_{1}} \Omega_{I_{2}} F M_{F} \Omega_{F}\right\rangle,
$$

which has been used to obtain Eq. (29).

Similarly, the long-range basis must also fulfill the Pauli principle. The long-range basis is defined in Eq. (14) and is a linear combination of basis states $\left|F_{1} F_{2} F_{A} M_{F_{a}}\right\rangle$ and $\left|l m_{l}\right\rangle$. The two atoms may or may not be in the same hyperfine state. Permuting the nuclei in the states $\left|F_{a} M_{F_{a}}\right\rangle$ gives

$$
\begin{aligned}
P_{n}\left|F_{1} F_{2} F_{a} M_{F_{a}}\right\rangle= & \sum_{M_{F_{1}}, M_{F_{2}}} \sum_{M_{S_{1}}, M_{I_{1}}} \sum_{M_{S_{2}}, M_{I_{2}}}\left\langle F_{1} M_{F_{1}} F_{2} M_{F_{2}} \mid F_{a} M_{F_{a}}\right\rangle \\
& \times\left\langle S_{1} M_{S_{1}} I_{1} M_{I_{1}} \mid F_{1} M_{F_{1}}\right\rangle \\
& \times\left\langle S_{1} M_{S_{2}} I_{1} M_{I_{2}} \mid F_{2} M_{F_{2}}\right\rangle P_{n}\left[\left|q S_{1} M_{S_{1}} S_{2} M_{S_{2}}\right\rangle\right. \\
& \left.\times\left|I_{1} M_{I_{1}} I_{2} M_{I_{2}}\right\rangle\right]
\end{aligned}
$$

To continue, we decouple the electronic spin $S$ in the sates $\left|q \Lambda S M_{S}\right\rangle$

$$
\left|q S_{1} M_{S_{1}} S_{2} M_{S_{2}}\right\rangle=\sum_{S, M_{S}}\left\langle S_{1} M_{S_{1}} S_{1} M_{S_{2}} \mid S M_{S}\right\rangle\left|q \Lambda S M_{S}\right\rangle
$$

and obtain $P_{n}\left|q S_{1} M_{S_{1}} S_{1} M_{S_{2}}\right\rangle$

$$
P_{n}\left|q S_{1} M_{S_{1}} S_{1} M_{S_{2}}\right\rangle=\sum_{S, M_{S}}\left\langle S_{1} M_{S_{1}} S_{1} M_{S_{2}} \mid S M_{S}\right\rangle(-1)^{i_{e}}\left|q \Lambda S M_{S}\right\rangle,
$$

where we have used Eq. (19) and $(-1)^{i_{e}}=(-1)^{S-2 S_{1}+N_{1}}$, which follows from Eq. (17) and requirement (18). Finally, we exploit the symmetry of the Clebsch-Gordan coefficients and write $\left\langle S_{1} M_{S_{1}} S_{2} M_{S_{2}} \mid S M_{S}\right\rangle=(-1)^{S_{1}+S_{2}-S}\left\langle S_{2} M_{S_{2}} S_{1} M_{S_{1}} \mid S M_{S}\right\rangle$ to obtain

$$
\begin{aligned}
P_{n}\left|q S_{1} M_{S_{1}} S_{2} M_{S_{2}}\right\rangle & =\sum_{S, M_{S}}\left\langle S_{2} M_{S_{2}} S_{1} M_{S_{1}} \mid S M_{S}\right\rangle(-1)^{N_{1}}\left|q \Lambda S M_{S}\right\rangle \\
& =(-1)^{N_{1}}\left|q S_{2} M_{S_{2}} S_{1} M_{S_{1}}\right\rangle
\end{aligned}
$$

The nuclear-spin states $\left|I_{1} M_{I_{1}} I_{1} M_{I_{2}}\right\rangle$ can be permuted without introducing any phase factor and we can conclude

$$
\begin{aligned}
P_{n}\left|F_{1} F_{2} F_{a} M_{F_{a}}\right\rangle= & \sum_{M_{F_{1}}, M_{F_{2}}} \sum_{S_{S_{1}}, M_{I_{1}}} \sum_{S_{S_{2}}, M_{I_{2}}}\left\langle F_{1} M_{F_{1}} F_{2} M_{F_{2}} \mid F_{a} M_{F_{a}}\right\rangle \\
& \times\left\langle S_{1} M_{S_{1}} I_{1} M_{I_{1}} \mid F_{1} M_{F_{1}}\right\rangle\left\langle S_{1} M_{S_{2}} I_{1} M_{I_{2}} \mid F_{2} M_{F_{2}}\right\rangle \\
& \times(-1)^{N_{1}}\left|q S_{2} M_{S_{2}} S_{1} M_{S_{1}}\right\rangle\left|I_{2} M_{I_{2}} I_{1} M_{I_{1}}\right\rangle \\
= & (-1)^{N_{1}+F_{1}+F_{2}-F_{a}\left|F_{2} F_{1} F_{a} M_{F_{a}}\right\rangle}
\end{aligned}
$$

The phase factor $(-1)^{F_{1}+F_{2}-F_{a}}$ stems from a rearrangement of the Clebsch-Gordan coefficient. Finally, we determine the sign of the states $\left|l m_{l}\right\rangle$ under nuclear permutation

$$
P_{n}\left|l m_{l}\right\rangle=(-1)^{l}\left|l m_{l}\right\rangle,
$$

which follows from the properties of the spherical harmonics. All together, the $F F$-coupled basis states fulfill the equation

$$
P_{n}\left|F_{1} F_{2} F_{a} l F M_{F}\right\rangle=(-1)^{N_{1}+F_{1}+F_{2}-F_{a}+l}\left|F_{2} F_{1} F_{a} l F M_{F}\right\rangle,
$$

hence we can construct eigenstates for the $P_{n}$ operator, 


$$
\begin{aligned}
\left|F_{1} F_{2} F_{a} l F M_{F}\right\rangle= & \frac{1}{\sqrt{2}}\left[\left|F_{1} F_{2} F_{a} l F M_{F}\right\rangle\right. \\
& \left.+(-1)^{N_{1}+F_{2}+F_{2}-F_{a}+l+2 I_{1}}\left|F_{2} F_{1} F_{a} l F M_{F}\right\rangle\right] .
\end{aligned}
$$

The phase factor is in agreement with the expression obtained by Bo Gao [16] working with similar basis states.

\section{INTERACTIONS}

Consider again the diatomic Hamiltonian, now written in the abbreviated form

$$
H=-\frac{\hbar^{2}}{2 \mu} \frac{d^{2}}{d r^{2}}+H_{\mathrm{ev}}+H_{\mathrm{hf}}+H_{\mathrm{ss}}+H_{\mathrm{Z}}+H_{\mathrm{rot}},
$$

where $H_{\mathrm{ev}}$ denotes the electronic and vibrational contribution. $H_{\mathrm{hf}}$ represents the hyperfine operator, $H_{\mathrm{Z}}$ is the Zeeman interaction operator, and $H_{\text {rot }}$ is the rotational energy operator. $H_{\mathrm{ss}}$ is the spin-spin interaction operator included explicitly. Other relativistic effects are included in $H_{\mathrm{ev}}$. In this section, we obtain the matrix elements for these operators in the molecular Hund's case (a) basis. Working with the case (a) basis enables rather compact expressions for the relevant matrix elements. The CPU time is also reduced by avoiding the all too familiar Clebsch-Gordan coefficients often present in large numbers in both hyperfine and Zeeman interaction terms. Furthermore, it is very easy to write down the different matrices in this basis, yielding an easy and transparent algorithm less susceptible to errors.

\section{A. Diatomic magnetic hyperfine interaction in Hund's case (a) basis}

The general diatomic hyperfine Hamiltonian is given in [31]. Only interactions that depend on the electronic spin are of current interest. We will start with a microscopic hyperfine Hamiltonian that takes the form

$$
H_{\mathrm{hf}}=\sum_{j=1}^{2} \sum_{\mu=-1}^{1}(-1)^{\mu} I_{j-\mu} \sum_{i=1}^{N} s_{i \mu} a\left(\mathbf{r}_{i j}\right),
$$

where $N$ is the total number of electrons. $I_{j-\mu}$ and $s_{i \mu}$, respectively, denote spherical components of the nuclear spin $\mathbf{I}_{j}$ and the electronic spin $\mathbf{s}_{i}$. The quantity $a\left(\mathbf{r}_{i j}\right)$ depends on the position of electron $i$ relative to nucleus $j$. The Hamiltonian in Eq. (40), in particular, applies to the dominant Fermicontact interaction, with $a\left(\mathbf{r}_{i j}\right)$ defined by

$$
a\left(\mathbf{r}_{i j}\right)=\frac{16 \pi}{3} g_{I_{j}} \mu_{0} \mu_{N} \delta\left(\mathbf{r}_{i j}\right)
$$

where $g_{I_{j}}$ denotes the $g$ factor of nucleus $j$.

We are considering the states $\left|q S \Sigma \Omega_{I_{1}} \Omega_{I_{2}}\right\rangle$ and have omitted $\left|F M_{F} \Omega\right\rangle$ as the hyperfine interaction is diagonal in the corresponding quantum numbers. $\Lambda$ is also ignored as it is zero in alkali-metal diatomic molecules (or it may be included in the collective quantum number $q$ ). The relevant matrix element can then be written as

$$
\begin{aligned}
\langle q S \Sigma & \left.\Omega_{I_{1}} \Omega_{I_{2}}\left|H_{\mathrm{hf}}\right| q^{\prime} S^{\prime} \Sigma^{\prime} \Omega_{I_{1}}^{\prime} \Omega_{I_{2}}^{\prime}\right\rangle \\
= & \sum_{\mu=-1}^{1}(-1)^{\mu}\left\langle\Omega_{I_{1}}\left|I_{1-\mu}\right| \Omega_{I_{1}}^{\prime}\right\rangle\langle q S \Sigma| \sum_{i=1}^{N} s_{i \mu} a\left(\mathbf{r}_{i 1}\right) \\
& \times\left|q^{\prime} S^{\prime} \Sigma^{\prime}\right\rangle \delta_{\Omega_{I_{2}}, \Omega_{I_{2}}^{\prime}}+\sum_{\mu=-1}^{1}(-1)^{\mu}\left\langle\Omega_{I_{2}}\left|I_{2-\mu}\right| \Omega_{I_{2}}^{\prime}\right\rangle \\
& \times\left\langle q S \Sigma\left|\sum_{i=1}^{N} s_{i \mu} a\left(\mathbf{r}_{i 2}\right)\right| q^{\prime} S^{\prime} \Sigma^{\prime}\right\rangle \delta_{\Omega_{I_{1}}, \Omega_{I_{1}}^{\prime}} .
\end{aligned}
$$

For the elements diagonal in spin $\left(S=S^{\prime}\right)$, it follows from Eq. (42) and the Wigner-Eckart theorem

$$
\begin{aligned}
& \left\langle q S \Sigma\left|\sum_{i=1}^{N} s_{i \mu} a\left(\mathbf{r}_{i 1}\right)\right| q S \Sigma^{\prime}\right\rangle \\
& =\sum_{i=1}^{N}\left\langle S \Sigma\left|s_{i \mu}\right| S \Sigma^{\prime}\right\rangle\left\langle q S \Sigma^{\prime}\left|a\left(\mathbf{r}_{i 1}\right)\right| q S \Sigma^{\prime}\right\rangle \\
& =\left\langle S \Sigma\left|S_{\mu}\right| S \Sigma^{\prime}\right\rangle \sum_{i=1}^{N}\left\langle S|| s_{i}|| S\right\rangle\left\langle q S \Sigma^{\prime}\left|a\left(\mathbf{r}_{i 1}\right)\right| q S \Sigma^{\prime}\right\rangle \\
& =\left\langle S \Sigma|S| S \Sigma_{\mu}^{\prime}\right\rangle b_{1},
\end{aligned}
$$

where $b_{1}$ has been defined as

$$
b_{1} \equiv \sum_{i=1}^{N}\left\langle S|| s_{i}|| S\right\rangle\left\langle q S \Sigma^{\prime}\left|a\left(\mathbf{r}_{i 1}\right)\right| q S \Sigma^{\prime}\right\rangle,
$$

and a similar definition holds for $b_{2}$ related to the other nucleus. $S_{\mu}$ is the spherical component $\mu$ of the total electronic spin. For two identical nuclei $b_{1}=b_{2}=b$. Combining Eqs. (42)-(44), the hyperfine interaction diagonal in $S$ can be written as

$$
\begin{aligned}
\langle q S \Sigma & \left.\Omega_{I_{1}} \Omega_{I_{2}}\left|H_{\mathrm{hf}}\right| q S \Sigma^{\prime} \Omega_{I_{1}}^{\prime} \Omega_{I_{2}}^{\prime}\right\rangle \\
= & b\left(\left\langle q S \Sigma \Omega_{I_{1}} \Omega_{I_{2}}\left|\mathbf{I}_{1} \cdot \mathbf{S}\right| q S \Sigma^{\prime} \Omega_{I_{1}}^{\prime} \Omega_{I_{2}}^{\prime}\right\rangle \delta_{\Omega_{I_{2}}, \Omega_{I_{2}}^{\prime}}\right. \\
& \left.\quad+\left\langle q S \Sigma \Omega_{I_{1}} \Omega_{I_{2}}\left|\mathbf{I}_{2} \cdot \mathbf{S}\right| q S \Sigma^{\prime} \Omega_{I_{1}}^{\prime} \Omega_{I_{2}}^{\prime}\right\rangle \delta_{\Omega_{I_{1}}, \Omega_{I_{1}}^{\prime}}\right),
\end{aligned}
$$

where the matrix elements in Eq. (45) are defined by

$$
\begin{aligned}
\langle q S \Sigma & \left.\Omega_{I_{1}} \Omega_{I_{2}}\left|\mathbf{I}_{j} \cdot \mathbf{S}\right| q S \Sigma^{\prime} \Omega_{I_{1}^{\prime}}^{\prime} \Omega_{I_{2}}^{\prime}\right\rangle \\
= & \Sigma \Omega_{I_{j}} \delta_{\Sigma, \Sigma^{\prime}} \delta_{\Omega_{I_{j}}, \Omega_{I_{j}}^{\prime}}+\frac{1}{2}[S(S+1)-\Sigma(\Sigma-1)]^{1 / 2} \\
& \times\left[I_{j}\left(I_{j}+1\right)-\Omega_{I_{j}}\left(\Omega_{I_{j}}+1\right)\right]^{1 / 2} \delta_{\Sigma^{\prime}, \Sigma-1} \delta_{\Omega_{I_{j}^{\prime}}^{\prime}, \Omega_{I_{j}}+1} \\
& +\frac{1}{2}[S(S+1)-\Sigma(\Sigma+1)]^{1 / 2} \\
& \times\left[I_{j}\left(I_{j}+1\right)-\Omega_{I_{j}}\left(\Omega_{I_{j}}-1\right)\right]^{1 / 2} \delta_{\Sigma^{\prime}, \Sigma+1} \delta_{\Omega_{I_{j}^{\prime}, \Omega_{I_{j}}-1},}
\end{aligned}
$$

with $j=1,2$. Equation (46) has been worked out by standard ladder operator techniques.

We now return to Eq. (42) and to the part of the hyperfine interaction off diagonal in $S$. Again, using the Wigner-Eckart theorem, 


$$
\begin{aligned}
&\left\langle q S \Sigma\left|\sum_{i=1}^{N} s_{i \mu} a\left(\mathbf{r}_{i 1}\right)\right| q^{\prime} S^{\prime} \Sigma^{\prime}\right\rangle= \sum_{i=1}^{N}\left\langle q S \Sigma\left|s_{i \mu}\right| q^{\prime} S^{\prime} \Sigma^{\prime}\right\rangle\langle q S \Sigma| a\left(\mathbf{r}_{i 1}\right) \\
& \times\left|q^{\prime} S^{\prime} \Sigma^{\prime}\right\rangle, \\
&=\left\langle 1 \mu S^{\prime} \Sigma^{\prime} \mid S \Sigma\right\rangle \sum_{i=1}^{N}\left\langle S|| s_{i}|| S^{\prime}\right\rangle\left\langle q\left|a\left(\mathbf{r}_{i 1}\right)\right| q^{\prime}\right\rangle=g_{1}\left\langle 1 \mu S^{\prime} \Sigma^{\prime} \mid S \Sigma\right\rangle
\end{aligned}
$$

where $g_{1}$ has been defined

$$
\begin{aligned}
g_{1} & \equiv \frac{1}{\left\langle 1 \mu S^{\prime} \Sigma^{\prime} \mid S \Sigma\right\rangle}\left\langle q S \Sigma\left|\sum_{i=1}^{N} s_{i \mu} a\left(\mathbf{r}_{i 1}\right)\right| q^{\prime} S^{\prime} \Sigma^{\prime}\right\rangle \\
& =\sum_{i=1}^{N}\left\langle S|| s_{i}|| S^{\prime}\right\rangle\left\langle q\left|a\left(\mathbf{r}_{i 1}\right)\right| q^{\prime}\right\rangle .
\end{aligned}
$$

A similar expression applies to $g_{2}$ for the other nucleus. We will return to the explicit connection between $g_{1}$ and $g_{2}$ later on. We note, however, that $g_{1}$ and $g_{2}$ are independent of $\mu$. $\left\langle 1 \mu S^{\prime} \Sigma^{\prime} \mid S \Sigma\right\rangle$ denotes the Clebsch-Gordan coefficient from the Wigner-Eckart theorem that couples a spherical tensor of rank 1 to the electronic spin $S^{\prime}$ to yield $S$. The offdiagonal matrix elements in terms of $g_{1}$ and $g_{2}$ are written as [combining Eqs. (42) and (48)]

$$
\begin{aligned}
& \left\langle q S \Sigma \Omega_{I_{1}} \Omega_{I_{2}}\left|H_{\mathrm{hf}}\right| q S^{\prime} \Sigma^{\prime} \Omega_{I_{1}}^{\prime} \Omega_{I_{2}}^{\prime}\right\rangle \\
& =g_{1} \sum_{\mu=-1}^{1}(-1)^{\mu}\left\langle 1 \mu S^{\prime} \Sigma^{\prime} \mid S \Sigma\right\rangle\left\langle\Omega_{I_{1}}\left|I_{1-\mu}\right| \Omega_{I_{1}}^{\prime}\right\rangle \delta_{\Omega_{I_{2}}, \Omega_{I_{2}}^{\prime}} \\
& \quad+g_{2} \sum_{\mu=-1}^{1}(-1)^{\mu}\left\langle 1 \mu S^{\prime} \Sigma^{\prime} \mid S \Sigma\right\rangle\left\langle\Omega_{I_{2}}\left|I_{2-\mu}\right| \Omega_{I_{2}}^{\prime}\right\rangle \delta_{\Omega_{I_{1}}, \Omega_{I_{1}}^{\prime}} .
\end{aligned}
$$

The matrix elements in Eq. (50) are readily calculated, and the results are

$$
\left\langle\Omega_{I_{i}}\left|I_{i \pm 1}\right| \Omega_{I_{i}^{\prime}}^{\prime}\right\rangle=\mp \frac{1}{\sqrt{2}}\left[I_{i}\left(I_{i}+1\right)-\Omega_{I_{i}}\left(\Omega_{I_{i}} \mp 1\right)\right]^{1 / 2} \delta_{\Omega_{I_{i}^{\prime}, \Omega_{I_{i}} \mp 1},},
$$

$$
\left\langle\Omega_{I_{i}}\left|I_{i 0}\right| \Omega_{I_{i}}^{\prime}\right\rangle=\Omega_{I_{i}} \delta_{\Omega_{I_{i}^{\prime}}^{\prime}, \Omega_{I_{i}}}
$$

Finally, we specialize to the alkali atoms where $S_{1}=S_{2}$ $=\frac{1}{2}$ and $S \in\{0,1\}$. It is readily seen from Eq. (49) that $g_{1}$ and $g_{2}$ are independent of $\mu$. To simplify the calculation of $g_{1}$ and $g_{2}$, we set $\mu=1$ which yields $\langle 1100 \mid 11\rangle=1\left(S^{\prime}=0, \Sigma^{\prime}\right.$ $=0)$. The relation between $g_{1}$ and $g_{2}$ is obtained by decoupling the total electronic spin $S$,

$$
\begin{gathered}
|q, S=1, \Sigma=1\rangle=\left|q, S_{1}, \Sigma_{1}=1 / 2, S_{2}, \Sigma_{2}=1 / 2\right\rangle, \\
|q, S=0, \Sigma=0\rangle=\frac{1}{\sqrt{2}}\left(\left|q^{\prime}, S_{1}, \Sigma_{1}=1 / 2, S_{2}, \Sigma_{2}=-1 / 2\right\rangle\right. \\
\left.-\left|q^{\prime} S_{1} \Sigma_{1}=-1 / 2, S_{2}, \Sigma_{2}=1 / 2\right\rangle\right),
\end{gathered}
$$

and we obtain

$$
g_{1}=-\frac{1}{\sqrt{2}}\left\langle q S_{1}, \Sigma_{1}=1 / 2\left|\sum_{i=1}^{N} s_{i 1} a\left(\mathbf{r}_{i 1}\right)\right| q^{\prime} S_{1}, \Sigma_{1}^{\prime}=-1 / 2\right\rangle .
$$

In a similar way, we obtain for $g_{2}$

$$
g_{2}=\frac{1}{\sqrt{2}}\left\langle q S_{2} \Sigma_{2}=1 / 2\left|\sum_{i=1}^{N} s_{i 1} a\left(\mathbf{r}_{i 2}\right)\right| q^{\prime} S_{2}, \Sigma_{2}^{\prime}=-1 / 2\right\rangle,
$$

which means that we have $g_{1}=-g_{2}$. Thus, we see that just two hyperfine parameters are needed. For the ${ }^{3} \Sigma$ state the only parameter is $b$, and for the ${ }^{3} \Sigma-{ }^{1} \Sigma$ hyperfine interaction there is a single parameter $g_{1}=-g_{2}$.

\section{B. $A b$ initio calculation of the molecular hyperfine parameters}

First, we consider the Fermi-contact hyperfine interaction. The parameter $b_{1}$ of Eq. (44) is then denoted $b_{1 F}$, and in the case of two identical nuclei $b_{1 F}=b_{2 F}=b_{F}$. The Fermi-contact parameter $b_{F}$, which is independent of $\mu$, is from Eqs. (43) and (41) given by

$$
b_{F}=\frac{16 \pi}{3} g_{I} \mu_{0} \mu_{N} \frac{1}{\Sigma}\left\langle q S \Sigma\left|\sum_{i=1}^{N} s_{i z} \delta\left(\mathbf{r}_{i 1}\right)\right| q S \Sigma\right\rangle .
$$

The expressions for $b_{F}$ above applies to the interaction that is diagonal in the spin $\left(S=S^{\prime}\right)$, i.e., to the triplet state for the alkali molecules.

For the Fermi-contact interaction that is off diagonal in $S$, i.e., triplet-singlet interaction in the alkali case, the corresponding parameters $b_{1 F}^{\prime}=g_{1}$ and $b_{2 F}^{\prime}=g_{2}$ are obtained from Eq. (49). Specializing to the diatomic alkali molecules, we see from Eqs. (55) and (56) that $b_{1 F}^{\prime}=-b_{2 F}^{\prime}$, and

$$
\begin{aligned}
b_{F}^{\prime}=b_{1 F}^{\prime}= & -\frac{16 \pi}{3} g_{I} \mu_{0} \mu_{N} \frac{1}{\sqrt{2}}\left\langle q, S_{1}=\frac{1}{2}, \Sigma=\frac{1}{2}\right| \sum_{i=1}^{N} s_{i 1} \delta\left(\mathbf{r}_{i 1}\right) \\
& \times\left|q^{\prime} S_{1}=\frac{1}{2}, \Sigma_{1}^{\prime}=-\frac{1}{2}\right\rangle .
\end{aligned}
$$

For a diatomic molecule, there is furthermore an anisotropic spin-dependent hyperfine interaction that is generally included through the parameter $c$ (cf. [31]). In this case, the quantity $a\left(\mathbf{r}_{i j}\right)$ in the Hamiltonian of Eq. (40) will also depend on the component $\mu$. The Fermi-contact hyperfine parameters $b_{F}$ and $b_{F}^{\prime}$ were computed $a b$ initio for ${ }^{6} \mathrm{Li}_{2}$ for several internuclear separations. Extensive configuration interaction $(\mathrm{CI})$ calculations were performed to obtain $a b$ initio values of the parameters $b_{F}$ and $b_{F}^{\prime}$ in ${ }^{6} \mathrm{Li}_{2}$. The results are shown in Fig. 10, with a corresponding discussion in Sec. VI C 1.

The anisotropic part of the diatomic hyperfine Hamiltonian yields two additional hyperfine parameters. For interactions diagonal in the spin $S$, there is an extra parameter $c$, and terms off diagonal in $S$ (singlet-triplet interaction) introduces a parameter $c^{\prime}$ (cf. [31]). Basically, the anisotropic part 
adds corrections to the Fermi-contact parameters $b_{F}$ and $b_{F}^{\prime}$ in the matrix elements of the hyperfine Hamiltonian as follows:

$$
\begin{gathered}
b_{F} \rightarrow b_{F}+\frac{2}{3} c, \quad \mu=0, \\
b_{F} \rightarrow b_{F}-\frac{1}{3} c, \quad \mu= \pm 1, \\
b_{F}^{\prime} \rightarrow b_{F}^{\prime}+\frac{2}{3} c^{\prime}, \quad \mu=0, \\
b_{F}^{\prime} \rightarrow b_{F}^{\prime}-\frac{1}{3} c^{\prime}, \quad \mu= \pm 1 .
\end{gathered}
$$

$A b$ initio values for the parameters $c$ and $c^{\prime}$ were obtained from calculations similar to those performed to obtain values for $b_{F}$ and $b_{F}^{\prime}$. The results are shown in Fig. 10 for the ${ }^{6} \mathrm{Li}_{2}$ molecule. We notice that the contribution from the anisotropic hyperfine interaction is rather insignificant compared with the dominant Fermi-contact interaction.

\section{Diatomic molecular Zeeman interaction in Hund's case (a) basis}

Calculations similar to those presented in this section can be found in [32], although this reference only considers diatomic molecules with one nuclear spin $I$. We extend the theory to include atoms with two nuclear spins $I_{1}$ and $I_{2}$, but we ignore some of the smaller second-order effects treated in [32]. We write the Zeeman Hamiltonian as

$$
H_{\mathrm{Z}}=\mu_{0} g_{S} \mathbf{S} \cdot \mathbf{B}=\mu_{0} g_{S} \sum_{\mu=-1}^{1}(-1)^{\mu} S_{\mu} B_{-\mu},
$$

and neglect the terms $\mu_{0}\left(g_{I} \mathbf{I}+g_{N} \mathbf{N}\right) \cdot \mathbf{B}\left(\mu_{0}\right.$ is the Bohr magneton), which are all expected to be much smaller than the electronic spin contribution. In Eq. (60), it has been assumed that $\mathbf{L}=0 . S_{\mu}$ and $B_{-\mu}$ refer to space-fixed spherical components. The space-fixed components $S_{\mu}$ are related to the molecule-fixed components $S_{\mu^{\prime}}^{\prime}$ by the usual $\mathcal{D}$ matrix elements

$$
S_{\mu}=\sum_{\mu^{\prime}} \mathcal{D}_{\mu \mu^{\prime}}^{1}(\phi, \theta, 0)^{*} S_{\mu^{\prime}}^{\prime}
$$

Inserted in Eq. (60), this gives the Hamiltonian

$$
H_{\mathrm{Z}}=\mu_{0} g_{S} \sum_{\mu, \mu^{\prime}=-1}^{1}(-1)^{\mu} \mathcal{D}_{\mu \mu^{\prime}}^{1}(\phi, \theta, 0)^{*} S_{\mu^{\prime}}^{\prime} B_{-\mu} .
$$

Labeling all quantum numbers except the total angular momentum $F$ by $w\left(|w\rangle=\left|q S \Sigma \Omega_{I_{1}} \Omega_{I_{2}}\right\rangle\right)$, the matrix elements are worked out as

$$
\begin{aligned}
\left\langle w F M \Omega_{F}\left|H_{Z}\right| w^{\prime} F^{\prime} M^{\prime} \Omega_{F}^{\prime}\right\rangle & \\
= & \mu_{0} g_{S} \sum_{\mu, \mu^{\prime}=-1}^{1}(-1)^{\mu}\left\langle w\left|S_{\mu^{\prime}}^{\prime} B_{-\mu}\right| w^{\prime}\right\rangle \\
& \quad \times\left\langle F M_{F} \Omega_{F}\left|\mathcal{D}_{\mu, \mu^{\prime}}^{1}(\phi, \theta, 0)^{*}\right| F^{\prime} M_{F}^{\prime} \Omega_{F}^{\prime}\right\rangle .
\end{aligned}
$$

The integral $\left\langle w\left|\mathcal{D}^{1}(\phi, \theta, 0)^{*}\right| w^{\prime}\right\rangle$ is easily evaluated as an integral over the product of three $\mathcal{D}$ matrices [cf. Eq. (26)]. By convention we assume that the magnetic field is along the space-fixed $z$ axis with magnetic field strength $B$. Thus, there is only a contribution from $\mu=0$ in Eq. (63) and we obtain

$$
\begin{aligned}
\left\langle w F M_{F} \Omega_{F}\left|H_{Z}\right| w^{\prime} F^{\prime} M_{F}^{\prime} \Omega_{F}^{\prime}\right\rangle & \\
= & B \mu_{0} g_{S} \sqrt{(2 F+1)\left(2 F^{\prime}+1\right)} \sum_{\mu^{\prime}=-1}^{1}\left(\begin{array}{ccc}
F & 1 & F^{\prime} \\
M_{F} & 0 & -M_{F}
\end{array}\right) \\
& \times\left(\begin{array}{ccc}
F & 1 & F^{\prime} \\
\Omega_{F} & -\mu^{\prime} & -\Omega_{F}^{\prime}
\end{array}\right)(-1)^{M_{F}-\Omega_{F}^{\prime}-\mu^{\prime}}\left\langle w\left|S_{\mu^{\prime}}^{\prime}\right| w^{\prime}\right\rangle \delta_{M_{F}, M_{F}^{\prime}} .
\end{aligned}
$$

As expected, it is clear from Eq. (64) that different $M_{F}$ quantum numbers are not mixed. After a short piece of algebra, we obtain the matrix elements with $F^{\prime}=F$,

$$
\begin{aligned}
& \left\langle w F M_{F} \Omega_{F}\left|H_{Z}\right| w F M_{F} \Omega_{F}\right\rangle_{\mu^{\prime}=0}=B \mu_{0} g_{s} \frac{M_{F} \Omega_{F}}{F(F+1)} \Sigma, \\
& \left\langle w F M_{F} \Omega_{F}\left|H_{Z}\right| w^{\prime} F M_{F} \Omega_{F}^{\prime}\right\rangle_{\mu^{\prime}= \pm 1} \\
& =-B \mu_{0} g_{s} \frac{M_{F} \sqrt{\left(F \pm \Omega_{F}\right)\left(F \mp \Omega_{F}+1\right)}}{2 F(F+1)}\left\langle w\left|S_{ \pm 1}^{\prime}\right| w^{\prime}\right\rangle \\
& \quad \times \delta_{\Omega_{F}^{\prime}, \Omega_{F} \mp 1},
\end{aligned}
$$

and for $F^{\prime}=F+1$,

$$
\begin{gathered}
\left\langle w F M_{F} \Omega_{F}\left|H_{\mathrm{Z}}\right| w(F+1) M_{F} \Omega_{F}\right\rangle_{\mu^{\prime}=0}=-B \mu_{0} g_{s} \sqrt{\frac{\left(F-\Omega_{F}+1\right)\left(F+\Omega_{F}+1\right)\left(F-M_{F}+1\right)\left(F+M_{F}+1\right)}{(F+1)^{2}(2 F+1)(2 F+3)}} \Sigma, \quad(67) \\
\left\langle w F M_{F} \Omega_{F}\left|H_{\mathrm{Z}}\right| w^{\prime}(F+1) M_{F} \Omega_{F}^{\prime}\right\rangle_{\mu^{\prime}= \pm 1}= \pm B \mu_{0} g_{s} \sqrt{\frac{\left(F-M_{F}+1\right)\left(F+M_{F}+1\right)\left(F \mp \Omega_{F}+1\right)\left(F \mp \Omega_{F}+2\right)}{(2 F+2)^{2}(2 F+1)(2 F+3)}}\left\langle w\left|S_{ \pm 1}^{\prime}\right| w^{\prime}\right\rangle \delta_{\Omega_{F}^{\prime}, \Omega_{F}+1},
\end{gathered}
$$

finally for $F=F-1$, 


$$
\begin{gathered}
\left\langle w F M_{F} \Omega_{F}\left|H_{\mathrm{Z}}\right| w(F-1) M_{F} \Omega_{F}\right\rangle_{\mu^{\prime}=0}= \\
-B \mu_{0} g_{s} \frac{\sqrt{F^{2}-M_{F}^{2}}}{F} \sqrt{\frac{F^{2}-\Omega_{F}^{2}}{(2 F-1)(2 F+1)}} \Sigma, \\
\left\langle w F M_{F} \Omega_{F}\left|H_{Z}\right| w^{\prime}(F-1) M_{F} \Omega_{F}^{\prime}\right\rangle_{\mu^{\prime}= \pm 1} \\
=\mp B \mu_{0} g_{s} \frac{\sqrt{F^{2}-M_{F}^{2}}}{2 F} \\
\times \sqrt{\frac{\left(F \pm \Omega_{F}-1\right)\left(F \pm \Omega_{F}\right)}{(2 F-1)(2 F+1)}}\left\langle w\left|S_{ \pm 1}^{\prime}\right| w^{\prime}\right\rangle \delta_{\Omega_{F}^{\prime}, \Omega_{F}^{\mp 1}} .
\end{gathered}
$$

Deriving these matrix elements from Eq. (64), it is important to be aware that the total angular momentum $\mathbf{F}$ has components that obey anomalous commutation relations [33]. For completeness, we also give

$$
\begin{aligned}
\left\langle w\left|S_{ \pm 1}^{\prime}\right| w^{\prime}\right\rangle= & \left\langle q S \Sigma \Omega_{I_{1}} \Omega_{I_{2}}\left|S_{ \pm 1}^{\prime}\right| q^{\prime} S^{\prime} \Sigma^{\prime} \Omega_{I_{1}}^{\prime} \Omega_{I_{2}}^{\prime}\right\rangle \\
= & \sqrt{S(S+1)-\Sigma(\Sigma \mp 1)} \\
& \times \delta_{q, q^{\prime}} \delta_{S, S^{\prime}} \delta_{\Omega_{I_{1}}, \Omega_{I_{1}}^{\prime}} \delta_{\Omega_{I_{2}}, \Omega_{I_{2}}^{\prime}} \delta_{\Sigma^{\prime}, \Sigma \mp 1} .
\end{aligned}
$$

\section{Diatomic molecular rotational interaction in Hund's case} (a) basis

The rotational energy operator is simply written $B(r) \mathbf{R}^{2}$ with $B(r)=\frac{\hbar^{2}}{2 \mu r^{2}}$, where $\mathbf{R}$ is the orbital angular momentum of the relative motion of the nuclei $A$ and $B$. However, the case (a) states are not eigenstates for this operator and the Hamiltonian is rewritten as

$$
\begin{aligned}
H_{\mathrm{rot}} & =B(r)[\mathbf{F}-(\mathbf{L}+\mathbf{S}+\mathbf{I})]^{2} \\
& =B(r)\left(\mathbf{F}-\mathbf{F}_{a}\right)^{2}=B(r)\left(\mathbf{F}^{2}-2 \mathbf{F} \cdot \mathbf{F}_{a}+\mathbf{F}_{a}^{2}\right) .
\end{aligned}
$$

$H_{\text {rot }}$ is in general diagonal in the quantum numbers $q, S, I_{1}, I_{2}, F, M_{F}$ but off diagonal in $\Omega(\Omega=\Sigma+\Lambda), \Omega_{I_{1}}$, and $\Omega_{I_{2}}$.
To work out the diagonal contribution, it is convenient to define $\mathbf{J}_{a} \equiv \mathbf{L}+\mathbf{S}$. Consider

$$
\begin{aligned}
\left\langle\Omega \Omega_{I_{1}} \Omega_{I_{2}}\left|\mathbf{F}_{a}^{2}\right| \Omega \Omega_{I_{1}} \Omega_{I_{2}}\right\rangle= & \left\langle\Omega \Omega_{I_{1}} \Omega_{I_{2}}\left|\left(\mathbf{J}_{a}+\mathbf{I}_{1}+\mathbf{I}_{2}\right)^{2}\right| \Omega \Omega_{I_{1}} \Omega_{I_{2}}\right\rangle \\
= & \left\langle\Omega \Omega_{I_{1}} \Omega_{I_{2}}\left|\mathbf{J}_{a}^{2}\right| \Omega \Omega_{I_{1}} \Omega_{I_{2}}\right\rangle+I_{1}\left(I_{1}+1\right) \\
& +I_{2}\left(I_{2}+1\right)+2 \Omega_{I_{1}} \Omega_{I_{2}}+2 \Omega\left(\Omega_{I_{1}}\right. \\
& \left.+\Omega_{I_{2}}\right) .
\end{aligned}
$$

The remaining element in Eq. (73) can be worked out: $\left\langle\Omega \Omega_{I_{1}} \Omega_{I_{2}}\left|\mathbf{J}_{a}^{2}\right| \Omega \Omega_{I_{1}} \Omega_{I_{2}}\right\rangle=\left\langle\Omega \Omega_{I_{1}} \Omega_{I_{2}}\left|\mathbf{L}^{2}+2 \mathbf{L} \cdot \mathbf{S}\right| \Omega \Omega_{I_{1}} \Omega_{I_{2}}\right\rangle+S(S$ $+1)$. Effectively, this gives $S(S+1)$ when $L=0$. However, if $L \neq 0$ the terms $\left\langle\Omega \Omega_{I_{1}} \Omega_{I_{2}}\left|\mathbf{L}^{2}+2 \mathbf{L} \cdot \mathbf{S}\right| \Omega \Omega_{I_{1}} \Omega_{I_{2}}\right\rangle$ can be included in $H_{\mathrm{ev}}$. Hence, we obtain for the diagonal matrix element

$$
\begin{aligned}
\left\langle\Omega \Omega_{I_{1}} \Omega_{I_{2}}\left|H_{\text {rot }}\right| \Omega \Omega_{I_{1}} \Omega_{I_{2}}\right\rangle= & B(r)\left[F(F+1)-2\left(\Omega+\Omega_{I_{1}}+\Omega_{I_{2}}\right)^{2}\right. \\
& +S(S+1)+I_{1}\left(I_{1}+1\right)+I_{2}\left(I_{2}+1\right) \\
& \left.+2 \Omega_{I_{1}} \Omega_{I_{2}}+2 \Omega\left(\Omega_{I_{1}}+\Omega_{I_{2}}\right)\right] . \quad(74)
\end{aligned}
$$

Next we turn to the off-diagonal elements. Consider first the elements off diagonal in both $\Omega_{I_{1}}$ and $\Omega_{I_{2}}$. The only relevant operator in this case is $2 B(r) \mathbf{I}_{1} \cdot \mathbf{I}_{2}$ and the corresponding matrix element is

$$
\begin{aligned}
2 B(r) & \left\langle\Omega \Omega_{I_{1}} \Omega_{I_{2}}\left|\mathbf{I}_{1} \cdot \mathbf{I}_{2}\right| \Omega \Omega_{I_{1}}^{\prime} \Omega_{I_{2}}^{\prime}\right\rangle \\
= & B(r) \sqrt{\left(I_{1}+\Omega_{I_{1}}\right)\left(I_{1}-\Omega_{I_{1}}+1\right)\left(I_{2}+\Omega_{I_{2}}+1\right)\left(I_{2}-\Omega_{I_{2}}\right)} \\
& \times \delta_{\Omega_{I_{1}}^{\prime}, \Omega_{I_{1}-1} \delta_{\Omega_{I_{2}}, \Omega_{I_{2}}+1}+B(r)} \\
& \times \sqrt{\left(I_{1}-\Omega_{I_{1}}\right)\left(I_{1}+\Omega_{I_{1}}+1\right)\left(I_{2}+\Omega_{I_{2}}\right)\left(I_{2}-\Omega_{I_{2}}+1\right)} \\
& \times \delta_{\Omega_{I_{1}^{\prime}, \Omega_{I_{1}}}^{\prime} \delta_{\Omega_{I_{2}}^{\prime}, \Omega_{I_{2}}-1} .}
\end{aligned}
$$

The operator $-2 B(r) \mathbf{F}_{a} \cdot \mathbf{F}$ will yield matrix elements off diagonal in $\Omega_{I_{1}}\left(\Omega_{I_{2}}\right)$ but diagonal in both $\Omega_{I_{2}}\left(\Omega_{I_{1}}\right)$ and $\Omega$. Keeping in mind that $\mathbf{F}$ obeys anomalous commutation relations, we obtain

$$
-2 B(r)\left\langle\Omega \Omega_{I_{1}} \Omega_{I_{2}}\left|\mathbf{F}_{a} \cdot \mathbf{F}\right| \Omega \Omega_{I_{1}^{\prime}}^{\prime} \Omega_{I_{2}}\right\rangle=-B(r) \sqrt{\left(I_{1} \pm \Omega_{I_{1}}+1\right)\left(I_{1} \mp \Omega_{I_{1}}\right)\left(F \pm \Omega_{F}+1\right)\left(F \mp \Omega_{F}\right)} \delta_{\Omega_{I_{1}^{\prime}}^{\prime}, \Omega_{I_{1}} \pm 1},
$$

with a similar expression for the elements off diagonal in $\Omega_{I_{2}}$.

In Eq. (72) we also have additional operators of the type $2 B \mathbf{J}_{a} \cdot \mathbf{I}_{j}$ with $j=1,2$. The corresponding matrix elements are off diagonal in both $\Omega$ and $\Omega_{I_{i}}$,

$$
\begin{aligned}
& 2 B(r)\left\langle\Omega \Omega_{I_{i}} \Omega_{I_{j}}\left|\mathbf{J}_{a} \cdot \mathbf{I}_{i}\right| \Omega^{\prime} \Omega_{I_{i}^{\prime}}^{\prime} \Omega_{I_{j}}\right\rangle \\
& \quad=B(r)\left\langle\Omega\left|L_{ \pm}+S_{ \pm}\right| \Omega^{\prime}\right\rangle \sqrt{\left(I_{i} \pm \Omega_{I_{i}}+1\right)\left(I_{i} \mp \Omega_{I_{i}}\right)} \delta_{\Omega_{I_{i}^{\prime}}, \Omega_{I_{i}} \pm 1}
\end{aligned}
$$

with $j=1,2$ and $j \neq i$. The matrix element in Eq. (77) is eas- ily evaluated: $\left\langle\Omega\left|L_{ \pm}+S_{ \pm}\right| \Omega^{\prime}\right\rangle=\sqrt{S(S+1)-\Sigma(\Sigma \mp 1)} \delta_{\Sigma^{\prime}, \Sigma \mp 1}$, when $L=0$ is assumed.

Finally, we give the matrix elements off diagonal in $\Omega$ but diagonal in both $\Omega_{I_{1}}$ and $\Omega_{I_{2}}$ coupled by the operator $\mathbf{J}_{a} \cdot \mathbf{F}$,

$$
\begin{aligned}
-2 B\langle & \left.\Omega \Omega_{I_{1}} \Omega_{I_{2}}\left|\mathbf{J}_{a} \cdot \mathbf{F}\right| \Omega^{\prime} \Omega_{I_{1}} \Omega_{I_{2}}\right\rangle \\
= & -B \sqrt{S(S+1)-\Sigma(\Sigma \mp 1)} \\
& \times \sqrt{\left(F \mp \Omega_{F}+1\right)\left(F \pm \Omega_{F}\right)} \delta_{\Sigma^{\prime}, \Sigma \mp 1} .
\end{aligned}
$$

All other matrix elements for the rotational Hamiltonian are zero. 


\section{E. Diatomic ${ }^{3} \Sigma$ spin-spin interaction in Hund's case (a) basis}

The electronic spin-spin Hamiltonian takes the form [34]

$$
H_{\mathrm{ss}}=D\left(S_{z}^{2}-\frac{1}{3} \mathbf{S}^{2}\right)+E\left(S_{x}^{2}-S_{y}^{2}\right) .
$$

For ${ }^{3} \Sigma$ states, $E=0$ and $D$ is equal to twice the standard spin-splitting parameter $\lambda(r)$. Ab initio calculations of the spin-spin parameter $\lambda(r)$ seem to be nonstandard, an implementation of such a calculation is found in [35]. The spinspin interaction is diagonal in the basis $|q \Lambda S \Sigma\rangle$, and the matrix element can be written as

$$
\left\langle q S \Sigma\left|H_{\mathrm{ss}}\right| q S \Sigma\right\rangle=2 \lambda(r)\left[\Sigma^{2}-\frac{1}{3} S(S+1)\right] .
$$

If we omit the constant diagonal contribution equal to $-\frac{D}{3} S(S+1)$, the spin-spin matrix elements take the simple form,

$$
\left\langle q S \Sigma\left|H_{\mathrm{ss}}\right| q S \Sigma\right\rangle=2 \lambda(r) \Sigma^{2},
$$

i.e., $\Sigma= \pm 1$ are degenerate and shifted up or down in energy relative to states with $\Sigma=0$, depending on the sign of $\lambda(r)$.

\section{COMPUTATIONAL DETAILS}

To solve the coupled Eq. (5), we have implemented the log-derivative method of Johnson [18]. Our routine determines which basis states to include based on the input from the user. The basis should be chosen in such a way that the important interactions are well represented. The restrictions imposed by the Pauli principle are also important as nonexisting states would interact with the allowed states otherwise. Computationally, the Pauli principle is also convenient in the sense that it restricts the number of states included in the calculation and thereby the dimension of the matrices. The interactions are set up in the Hund's case (a) basis using the matrix elements worked out in the previous section. Interatomic potentials are necessary input along with values for the hyperfine parameters, which were handled in Sec. III B.

The various matrix elements in Eq. (5) must be evaluated at different values of $r$. However, the Zeeman interaction is independent of $r$ and the Zeeman matrix elements are set up only once. Similarly, the matrix elements of the rotational operator (72) depend on $r$ only through $B(r)$. This is in contrast with the hyperfine interaction which depends on $r$ in a more complex manner; hence the matrix elements repeatedly need to be updated. The spin-spin interaction is diagonal and depends on $r$ through $\lambda(r)$.

The integration of Eq. (5) extends from 2.75 to 300 a.u. Interpolation in the interatomic potentials, hyperfine interaction parameters, and the spin-spin parameter $\lambda(r)$ were performed with cubic splines. The reduced mass used for ${ }^{6} \mathrm{Li}$ is 5482.264721 a.u. calculated with the isotope mass obtained from [36].

The unitary transformation given in Sec. II A 3 is used to transform from Hund's case (a) basis to the $F F$-coupled basis. Finally, we perform another unitary transformation to a basis that diagonalizes both the hyperfine interaction and the
TABLE I. Comparison of the van der Waals coefficients $C_{6}, C_{8}, C_{10}$. All numbers in atomic units.

\begin{tabular}{lccc}
\hline \hline & Yan et al. $^{\mathrm{a}}$ & Zemke and Stwalley $^{\mathrm{b}}$ & Marinescu et $a .^{\mathrm{c}}$ \\
\hline$C_{6}$ & $1393.39(16)$ & $1381 \pm 8$ & 1388 \\
$C_{8}$ & $83425.8(4.2)$ & $82616 \pm 2288$ & 83240 \\
$C_{10}$ & $73721(1) \times 10^{2}$ & $(64250 \pm 5140) \times 10^{2}$ & $73650 \times 10^{2}$ \\
\hline \hline
\end{tabular}

${ }^{\mathrm{a}}$ Reference [44].
${ }^{\mathrm{b}}$ Reference [41].
${ }^{\mathrm{c}}$ Reference [45].

Zeeman interaction terms. This final transformation defines the $K$ matrix. From the output, we can extract the $S$ matrix and the scattering lengths, in principle, for any partial wave $l$.

\section{SINGLET AND TRIPLET INTERACTION POTENTIAL CURVES}

Reliable interaction potentials are very important in ultracold physics. Fortunately, the $\mathrm{Li}_{2}$ interatomic potentials have been extensively studied experimentally and theoretically, both for the ground state $X^{1} \Sigma_{g}^{+}$and for the lowest electronic triplet state $A^{3} \Sigma_{u}^{+}$.

For the $X^{1} \Sigma_{g}^{+} \mathrm{Li}_{2}$ interatomic potential, we use the $a b$ initio potential calculated by Zavitsas [37] for distances between $3.4 a_{0}$ and $12.0 a_{0}$. We extend the data of Zavitsas [37] with three values from Barakat et al. [38] at 12.6 $a_{0}, 13.4 a_{0}$, and $14.5 a_{0}$. In addition, we use two values from Konowalow and Olsen [39] at 2.75 $a_{0}$ and $3.00 a_{0}$, and a value of Schmidt Mink et al. [40] at $3.25 a_{0}$. The interatomic potential curve was smoothly joined with cubic splines to the long-range potential in Eq. (82).

For the ${ }^{3} \Sigma_{u}^{+}$state, we use the interatomic potential discussed by Zemke and Stwalley [41] for distances between $6.39 a_{0}$ and $15.5 a_{0}$. We have supplemented this potential with seven values (at $3.00 a_{0}, 3.50 a_{0}, 4.00 a_{0}, 4.50 a_{0}, 5.00 a_{0}$, $5.50 a_{0}$, and $6.00 a_{0}$ ) from Konowalow et al. [42]. Cubic splines were used to join the short- and long-range potentials.

At large separations, both the singlet and triplet interaction potentials can be written as a sum of dispersion terms [43]

$$
V(r)=-\frac{C_{6}}{r^{6}}-\frac{C_{8}}{r^{6}}-\frac{C_{10}}{r^{10}} .
$$

The dispersion coefficients (also called van der Waals coefficients) $C_{6}, C_{8}$, and $C_{10}$ for $\mathrm{Li}_{2}$ have been calculated by several authors. In Table I, we compare some of the most recent calculations which show rather good agreement. We have adopted the values obtained by Yan et al. for $C_{6}, C_{8}$, and $C_{10}$.

The adopted singlet and triplet interatomic potentials are shown in Fig. 1. The long-range potential was used for distances greater than $\simeq 16.5 a_{0}$.

With the combined short- and long-range interatomic potentials described in this section, we obtained the elastic $s$-wave scattering lengths $a_{s}^{T}=-2090.94 a_{0}$ and $a_{s}^{S}$ 


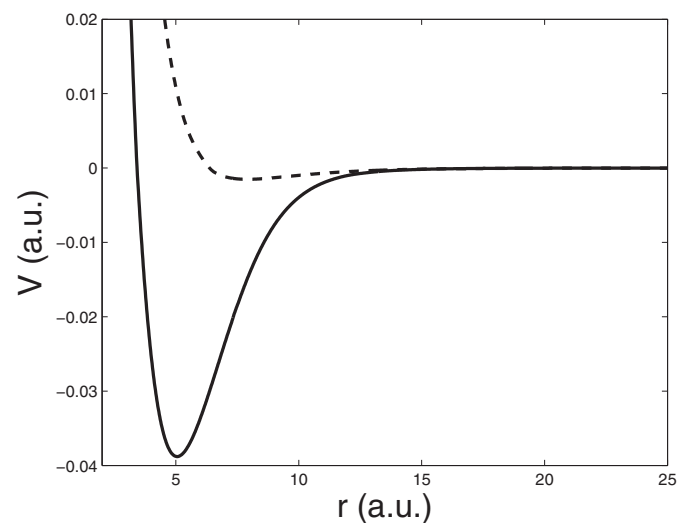

FIG. 1. Interatomic potential curves for the electronic states ${ }^{1} \Sigma_{g}^{+}$ (solid line) and ${ }^{3} \Sigma_{u}^{+}$in $\mathrm{Li}_{2}$.

$=45.5582 a_{0}$ for the triplet $(T)$ and singlet $(S)$ potentials, respectively. The singlet elastic-scattering length is experimentally determined with rather high accuracy to be $a_{s}^{S}$ $=45.1519(16) a_{0}$ [46], whereas the triplet scattering length is not that well known. Abraham et al. [47] reported the value $a_{s}^{T}=-2160 \pm 250 a_{0}$.

The different short- and long-range interatomic potentials available in the literature yield deviating results for the scattering lengths. Thus it seems hard to obtain ab initio scattering lengths with an accuracy comparable with the experimental values, without of course somehow fitting the combined (short and long range) potential to reproduce the experimental results. Ab initio scattering lengths are therefore very demanding to calculate. Without the experimental values available to guide the search for reliable interatomic potentials, $a_{s}^{S}$ and, in particular, $a_{s}^{T}$ would be reported with large uncertainties. One should also be aware that a completely inadequate interatomic potential can give excellent values for the scattering lengths; thus there are no one-to-one correspondence.

It has been reported previously that most $s$-wave Feshbach resonances observed in ${ }^{6} \mathrm{Li}$ are very sensitive to the singlet potential and rather insensitive to the triplet potential curve. An exception is the broad well-known Feshbach resonance at $B \simeq 837 \mathrm{G}$ (see next section), which is highly sensitive to both. Thus, the rather small difference between the $a b$ initio calculated $a_{s}^{S}$ compared to the experimental value may indicate that the calculated resonance positions are systematically shifted relative to the observed positions. We explore this further in the next section and we will see that this shift is roughly plus a few Gauss for all resonances.

\section{RESULTS}

\section{A. Calculation of $s$-wave scattering lengths and Feshbach resonances}

In this section, we report $s$-wave scattering lengths and Feshbach resonances for two colliding ${ }^{6} \mathrm{Li}$ atoms. The $s$-wave scattering length is defined as

$$
a_{s}=-\lim _{k \rightarrow 0} \frac{\tan \delta_{0}(k)}{k},
$$

where the phase shift $\delta_{0}$ has been defined in Eq. (12). In alkali-metal diatomic molecules where $L_{1}=L_{2}=0$, the molecular mechanical rotation quantum number $N$ corresponds to the partial-wave quantum number $l$. In the following, $N$ $=l$ and we write $N$ rather than $l$.

The ${ }^{6} \mathrm{Li}$ isotope has a nuclear spin $I_{1}=1$ and an electronic spin $S_{1}=1 / 2$ with a total angular momentum $\mathbf{F}_{1}=\mathbf{S}_{1}+\mathbf{I}_{1}$ $\in\left\{\frac{1}{2}, \frac{3}{2}\right\}$, i.e., two hyperfine levels for zero external magnetic field. The subscript 1 is used as a label to distinguish atom 1 from atom 2. Collisions can occur between atoms in the hyperfine levels $F_{1}=F_{2}=\frac{1}{2}, F_{1}=F_{2}=\frac{3}{2}$ and $F_{i}=\frac{1}{2}, F_{j}=\frac{3}{2},\{i, j\}$ $=1,2$ and $i \neq j$. Experimentally, most studied are collisions between atoms in the lowest-allowed energetic states $\left|F_{1} M_{F 1}\right\rangle=\left|\frac{1}{2}, \frac{1}{2}\right\rangle$ and $\left|F_{2} M_{F 2}\right\rangle=\left|\frac{1}{2},-\frac{1}{2}\right\rangle$. Collisions between atoms in excited states may be inelastic and give spin relaxation. We have calculated $s$-wave scattering lengths for all possible collisions, including atoms in different hyperfine levels. The results are presented in Fig. 3 for magnetic field strengths up to $1500 \mathrm{G}\left(1 \mathrm{G}=10^{-4} \mathrm{~T}\right)$.

The states are identified by the quantum numbers $N$ and $M_{F}$ and in addition a label $n$ to distinguish states with equal quantum numbers $N$ and $M_{F}$. The new notation is motivated by the fact that the quantum numbers $F_{1}, F_{2}, F_{a}$ and $F$ are generally mixed in external magnetic fields. Table II lists all relevant states $\left|n N M_{F}\right\rangle$ and their correspondence with the low- or zero-field states $\left|F_{1} F_{2} F M_{F}\right\rangle$. Since $N=0$, the quantum number $F_{a}=F$ and is suppressed in the notation. The possible molecular levels corresponding to $N=0$ (no mechanical rotation, lowest rotational level) are shown schematically in Fig. 2.

From the discussion in Sec. II A, it is clear that the states $\left|F_{1} F_{2} F M_{F}\right\rangle$ can be written as linear combinations of states

TABLE II. Correspondence between the states $\left|n, N, M_{F}\right\rangle$ and the zero-field states $\left|F_{1}, F_{2}, F, M_{F}\right\rangle . F_{1}, F_{2}$ and $F$ are good quantum numbers only at zero magnetic field.

\begin{tabular}{cccccc}
\hline \hline$\left|n N M_{F}\right\rangle$ & $\left|F_{1} F_{2} F M_{F}\right\rangle$ & $\left|n N M_{F}\right\rangle$ & $\left|F_{1} F_{2} F M_{F}\right\rangle$ & $\left|n N M_{F}\right\rangle$ & $\left|F_{1} F_{2} F M_{F}\right\rangle$ \\
\hline$|1,0,0\rangle$ & $\left|\frac{1}{2}, \frac{1}{2}, 0,0\right\rangle$ & $|1,0,-2\rangle$ & $\left|\frac{3}{2}, \frac{1}{2}, 2,-2\right\rangle$ & $|2,0,1\rangle$ & $\left|\frac{3}{2}, \frac{1}{2}, 2,1\right\rangle$ \\
$|2,0,0\rangle$ & $\left|\frac{3}{2}, \frac{1}{2}, 2,0\right\rangle$ & $|2,0,-2\rangle$ & $\left|\frac{3}{2}, \frac{3}{2}, 2,-2\right\rangle$ & $|3,0,1\rangle$ & $\left|\frac{3}{2}, \frac{1}{2}, 1,1\right\rangle$ \\
$|3,0,0\rangle$ & $\left|\frac{3}{2}, \frac{1}{2}, 1,0\right\rangle$ & $|1,0,2\rangle$ & $\left|\frac{3}{2}, \frac{3}{2}, 2,2\right\rangle$ & $|1,0,-1\rangle$ & $\left|\frac{3}{2}, \frac{1}{2}, 1,-1\right\rangle$ \\
$|4,0,0\rangle$ & $\left|\frac{3}{2}, \frac{1}{2}, 2,0\right\rangle$ & $|2,0,2\rangle$ & $\left|\frac{3}{2}, \frac{1}{2}, 2,2\right\rangle$ & $|2,0,-1\rangle$ & $\left|\frac{3}{2}, \frac{1}{2}, 2,-1\right\rangle$ \\
$|5,0,0\rangle$ & $\left|\frac{3}{2}, \frac{3}{2}, 0,0\right\rangle$ & $|1,0,1\rangle$ & $\left|\frac{3}{2}, \frac{3}{2}, 2,1\right\rangle$ & $|3,0,-1\rangle$ & $\left|\frac{3}{2}, \frac{3}{2}, 2,-1\right\rangle$ \\
\hline \hline
\end{tabular}




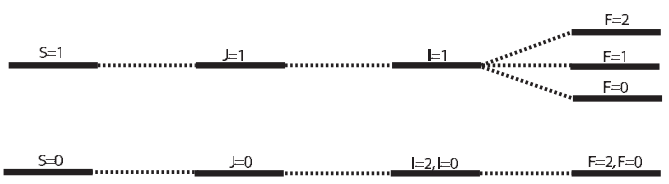

FIG. 2. Schematic diagram of the hyperfine energy levels in ${ }^{6} \mathrm{Li}_{2}$ consistent with $N=0$. Quantum numbers at short range in accordance with Hund's case (b) are indicated. Missing levels are excluded by the Pauli exclusion principle (e.g., all $N=I=0$ levels).

$\left|\phi_{n}\right\rangle$ which are eigenstates for the total electronic spin operator $\mathbf{S}^{2}=\left(\mathbf{S}_{1}+\mathbf{S}_{2}\right)^{2}$ [e.g., Hund's case (a) or Hund's case (b) states]

$$
\left|F_{1} F_{2} F M_{F}\right\rangle=\sum_{n} c_{m n}\left|\phi_{n}\right\rangle
$$

At zero field, none of the states $\left|n N M_{F}\right\rangle$ can be written as linear combinations of only singlet states $\left|\phi_{n}(S=0)\right\rangle$ as such
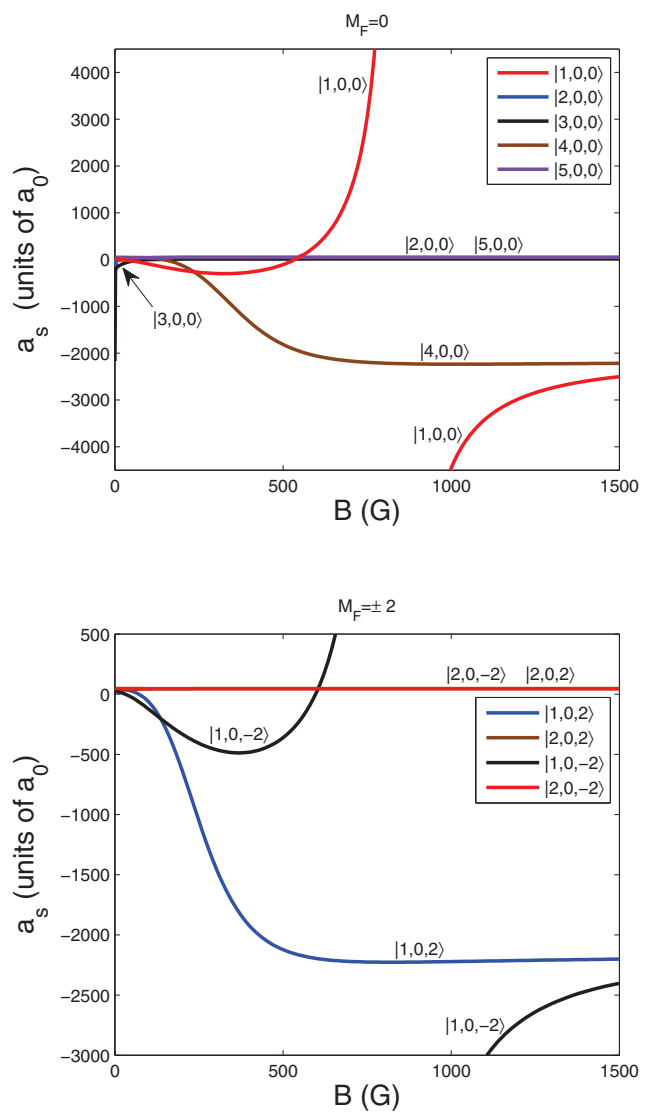

states would have zero hyperfine energy as $r \rightarrow \infty$. However, pure triplet states $\left|n N M_{F}\right\rangle$ do exist. In fact, at zero magnetic field the states $|3,0,0\rangle,|3,0,1\rangle$, and $|2,0,-1\rangle$ have total electronic spin $S=1$, and corresponding $s$-wave scattering lengths equal to $a_{s}^{T}$. As the magnetic field is increased from zero, singlet states are introduced in the linear combinations (84), and the sensitive $s$-wave scattering length changes very rapidly. This particular behavior is not resolved in Fig. 3.

The states $|4,0,0\rangle,|1,0,2\rangle$, and $|1,0,1\rangle$ have $s$-wave scattering lengths that show a steep decrease when the magnetic field is increased. At zero magnetic field, the scattering lengths are moderate, but at higher magnetic field strengths they attain the triplet scattering length $a_{s}^{T}$. In fact, at $B$ $\gtrsim 1000 \mathrm{G}$ these states are almost pure triplet states. To confirm, this we have plotted the sum of coefficients $\Sigma_{n} \mid c_{m n}(S$ $=1)\left.\right|^{2}$ vs magnetic field for the states $|4,0,0\rangle,|2,0,-1\rangle$, and $|2,0,2\rangle$ in Fig. 4. The states $|2,0,-1\rangle$ and $|2,0,2\rangle$ are also included for comparison and are seen to have rather different behaviors.

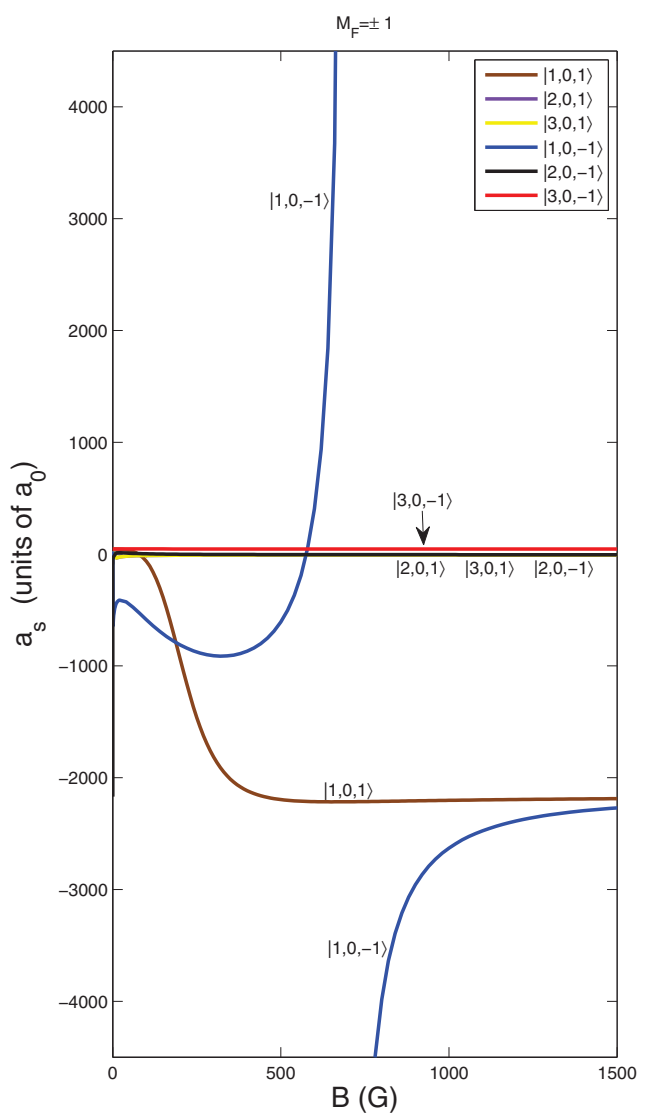

FIG. 3. (Color online) Scattering lengths ( $s$ wave) vs magnetic field $B$ for all states with $N=0$. Upper left panel includes all states with $M_{F}=0$, lower left panel includes all states with $M_{F} \pm 2$, whereas the right panel includes all states with $M_{F} \pm 1$. Each of the three panels shows one well-resolved Feshbach resonance. 


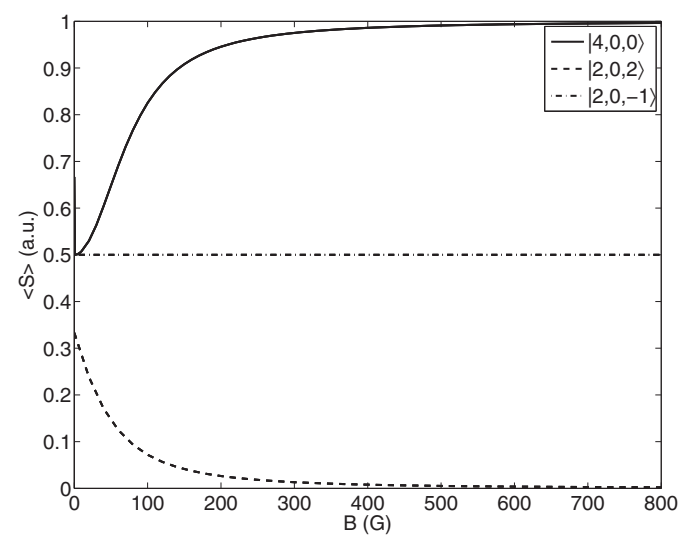

FIG. 4. $\langle S\rangle$ as a function of magnetic field for $|4,0,0\rangle$ and $|2,0,2\rangle$ (dotted line).

In general, the scattering length varies only weakly with the magnetic field, a property that is seen to hold for a majority of the states in Fig. 3. In fact, most states appear largely unaffected by the magnetic field, e.g., the states $|2,0,0\rangle,|2,0,2\rangle,|5,0,0\rangle,|3,0,-1\rangle,|2,0,1\rangle$, and $|2,0,-2\rangle$ all have moderate scattering lengths at all investigated magnetic field strengths. There are several reasons for this, some of which will be discussed later on.

The states $|3,0,1\rangle$ and $|2,0,-1\rangle$ share a particular property. Their energy eigenvalues are not affected by the increasing magnetic field strength. Both states are part of (different) three level systems of interacting states in which one of the eigenvalues retains a nearly constant value due to particular relations among the matrix elements for the hyperfine and Zeeman interactions. At large magnetic fields, both $|3,0,1\rangle$ and $|2,0,-1\rangle$ have scattering lengths of roughly $-6 a_{0}$ and are found to be in a 50/50 superposition of singlet and triplet basis states (see also Fig. 4).

Further, the states $|2,0,-2\rangle,|2,0,2\rangle$, and $|2,0,0\rangle$ have energy eigenvalues that approach zero when the magnetic field strength increases. Hence, in larger magnetic fields these states are almost pure singlet states with a moderate scattering length $a_{s}^{S}$. Finally, $|3,0,-1\rangle$ shares much of the same behavior, although the energy is decreasing toward zero more slowly.

The most prominent features in Fig. 3 are the three clearly visible Feshbach resonances, among them the broad wellknown ${ }^{6} \mathrm{Li}_{2}, F_{1}=F_{2}=\frac{1}{2}$ resonance in $|1,0,0\rangle$. All Feshbach resonances reported in the present paper originate when the hyperfine energy levels of the colliding atoms are Zeeman shifted into resonance with the highest vibrational level $(v$ =38) of the $X^{1} \Sigma_{g}^{+}$electronic state (the molecular ground state). The precise resonance positions are shifted by the hyperfine interaction and depend upon the state in question and its interacting partners. We remark that states where $F_{1}=F_{2}$ $=\frac{3}{2}$ (low-field quantum numbers) will not have Feshbach resonances as the corresponding energy eigenvalues are larger than zero even at the highest magnetic field strengths investigated. In the upper left panel of Fig. 3, we recognize the broad Feshbach resonance. In our calculation it is located at $B=837.13 \mathrm{G}$, experimentally it is found at $B_{0} \in[822$ -834] G [46]. Part of this discrepancy might be caused by the $a b$ initio singlet and triplet potential curves which do not reproduce the precise experimental scattering lengths. Similar discrepancies can be seen in the positions of all calculated Feshbach resonances. The $B \simeq 837 \mathrm{G}$ resonance is accessible through collisions of atoms in the states $\left|F_{1} M_{F_{1}}\right\rangle=\left|\frac{1}{2}, \frac{1}{2}\right\rangle$ and $\left|F_{2} M_{F_{2}}\right\rangle=\left|\frac{1}{2},-\frac{1}{2}\right\rangle$. This is a very broad resonance which makes it well suited for experiments as it is easy to control the scattering length with high precision.

Two additional broad Feshbach resonances are visible in Fig. 3 (lower left and right panels). In the lower left panel, we report a Feshbach resonance experimentally less accessible as it occurs in $|1,0,-1\rangle$ which is a linear combination [see Eq. (13)] of the $F F$-coupled states $\left|\frac{3}{2},-\frac{3}{2}, \frac{1}{2}, \frac{1}{2}\right\rangle$ and $\mid \frac{3}{2}$, $\left.-\frac{1}{2}, \frac{1}{2},-\frac{1}{2}\right\rangle$. The precise location of this Feshbach resonance is $B=692.89 \mathrm{G}$ in our calculation. Furthermore, we also find a Feshbach resonance in $|1,0,-2\rangle$ accessible through collisions of atomic pairs in the atomic states $\left(\left|F_{i}, M_{F_{i}}\right\rangle\right)\left|\frac{3}{2},-\frac{3}{2},\right\rangle$ and $\left|\frac{1}{2},-\frac{1}{2}\right\rangle$ (low-field quantum numbers), with a combined total angular-momentum projection $M_{F}=-2$. The resonance is clearly visible in Fig. 3 (lower left plot). A detailed calculation locates this resonance at $B=813.98 \mathrm{G}$.

In addition to the three $s$-wave resonances at $B$ $\simeq 690-840 \mathrm{G}$ discussed above, a narrow Feshbach resonance in the state $|1,0,0\rangle$ can be seen in Fig. 6 at the precise magnetic field strength $B=544.90 \mathrm{G}$. Experimentally, the resonance is observed at 543.28 $\pm 0.08 \mathrm{G}$ [46]. It has been reported that this particular resonance has a total nuclear spin $I=2$, whereas the broad $B \simeq 837 \mathrm{G}$ resonance has $I=0$ [46]. In contrast to $|1,0,0\rangle$, the states $|1,0,-1\rangle$ and $|1,0,-2\rangle$ do not have two Feshbach resonances as they do not interact with any $I=0$ states. However, at much larger field strengths the $v=37$ singlet vibrational level will become energetically accessible and make additional Feshbach resonances possible [48].

Figure 5 sums up the discussion of the $s$-wave scattering lengths as a function of magnetic field. States with corresponding energy eigenvalues that are easily tunable by magnetic fields will become pure triplet states in high magnetic fields with large and negative scattering lengths $\simeq a_{s}^{T}$. States with energy eigenvalues that are Zeeman shifted to lower energies may be tuned into resonance with the singlet $v$ $=38$ vibrational level and give rise to Feshbach resonances. However, most states are only weakly influenced by the applied field, with moderate scattering lengths that show small dependencies on the magnetic field strength.

\section{B. Calculation of the $\boldsymbol{p}$-wave scattering volume}

It is well known that $s$-wave collisions between two fermions in identical spin states are forbidden by the Pauli principle. However, $p$-wave collisions are possible although suppressed at ultracold temperatures as $\delta_{p} \propto E^{3 / 2}$, whereas $\delta_{s}$ $\propto E^{1 / 2}$. Whenever $s$-wave scattering is not allowed, the $p$-wave scattering volume $V_{p}$ replaces the usual $s$-wave scattering length as one of the important parameters. The $p$-wave scattering volume is defined as 


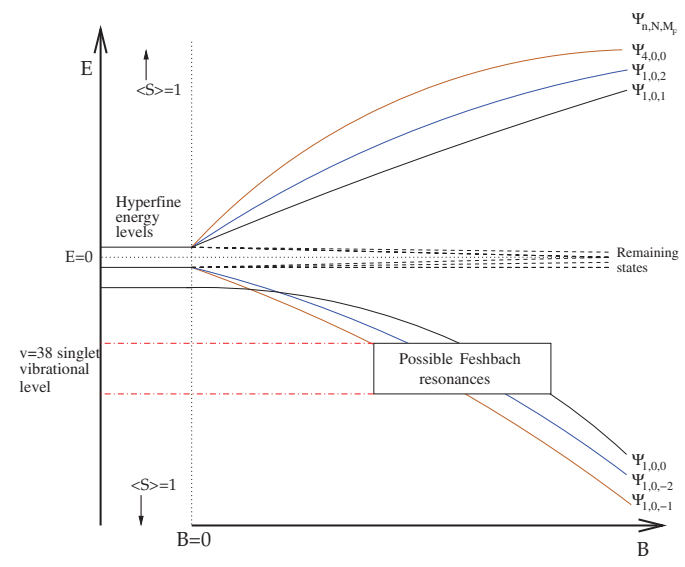

FIG. 5. (Color online) Schematic presentation of the Zeeman shift for different states as a function of the magnetic field strength. At zero field, there are only three hyperfine levels. At nonzero magnetic field, three states with different $M_{F}$ quantum numbers are shifted sufficiently low in energy to enable Feshbach resonances. The energy differences are not correctly displayed in this figure.

$$
V_{p} \equiv-\lim _{k \rightarrow 0} \frac{\tan \delta_{p}(k)}{k^{3}},
$$

and $a_{p}=\sqrt[3]{V_{p}}$ is sometimes referred to as the $p$-wave scattering length. $\delta_{p}$ is the $p$-wave phase shift and as usual $k$ is the wave number. To avoid the cube root, we report $V_{p}$ instead of $a_{p}$.

Knowledge of the $p$-wave scattering volume is in general of importance for experiments involving fermions. The $p$-wave scattering volume has also been discussed in relation with $p$-wave Cooper pairs [49] and $p$-wave scattering is an important mechanism in a three-body recombination of identical spin-polarized fermions [50].

We present calculations of the $p$-wave scattering volume as a function of magnetic field for selected spin states. We have focused on states where $s$-wave scattering is forbidden and restricted the calculations to states corresponding to the lowest atomic hyperfine level, that is, $F_{1}=F_{2}=\frac{1}{2}$. A sketch of molecular hyperfine levels corresponding to $N=1$ is presented in Fig. 7.

We have used the same singlet and triplet potentials as described in Sec. V. The corresponding singlet and triplet $p$-wave scattering volumes are $V_{p}^{S}=62298 a_{0}^{3}$ and $V_{p}^{T}=$ $-48252 a_{0}^{3}$, respectively. This yields $p$-wave scattering lengths $a_{p}^{S}=39.6 a_{0}$ and $a_{p}^{T}=-36.4 a_{0}$. The $a_{p}^{T}$ value should be compared to $-45 a_{0}$ obtained in [51].

$V_{p}$ as a function of $B$ is shown in Fig. 8. Because of the Pauli principle, the total atomic spin $F_{a}=F_{1}+F_{2}=1$ for all zero-field states. The definition and correspondence between the states $\left|n N M_{F}\right\rangle$ and the zero-field states $\left|F_{1} F_{2} F_{a} F M_{F}\right\rangle$ are given in Table III.

Three different Feshbach resonances are visible in Fig. 8, at the magnetic fields $B_{1}=162.99 \mathrm{G}, B_{2}=189.02 \mathrm{G}$, and $B_{3}=218.72 \mathrm{G}$. All states corresponding to the lowest hyper-

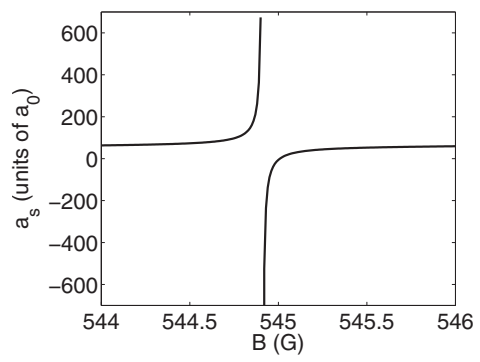

FIG. 6. Scattering length $a_{s}$ vs magnetic field showing a narrow Feshbach resonance in the $|1,0,0\rangle$ state at $B=544.90 \mathrm{G}$.

fine level $F_{1}=F_{2}=\frac{1}{2}$ at zero field show one of these resonances, which one depends on the detailed interaction, i.e., the response to the magnetic field.

There are three different $F F$-coupled states $\left|F_{1} M_{F_{1}} F_{2} M_{F_{2}}\right\rangle$ corresponding to $F_{1}=F_{2}=\frac{1}{2}$. These are $\left|\frac{1}{2}, \frac{1}{2}, \frac{1}{2}, \frac{1}{2}\right\rangle, \mid \frac{1}{2}, \frac{1}{2} \frac{1}{2}$, $\left.-\frac{1}{2}\right\rangle$, and $\left|\frac{1}{2},-\frac{1}{2}, \frac{1}{2},-\frac{1}{2}\right\rangle$. The magnetic field only interacts with the atomic spin; thus the corresponding energies should only depend on the quantum numbers $F_{1}, M_{F_{1}}, F_{2}, M_{F_{2}}$. The energy eigenvalues corresponding to the states $\left|F M_{F}\right\rangle$ will therefore be degenerate with only three distinct values at all magnetic field strengths. Figure 9 show the energy of all states as a function of magnetic fields $B \in[0,100] \mathrm{G}$.

Collisions between atoms with $M_{F_{1}}=M_{F_{2}}=\frac{1}{2}$ enable a $p$-wave Feshbach resonances at $B_{1}$, whereas collisions between atoms in $M_{F_{1}}=M_{F_{2}}=-\frac{1}{2}$ enables a $p$-wave Feshbach resonance at $B_{3}$. Finally, there exists a $p$-wave Feshbach resonance at $B_{2}$ from collisions between atoms in $M_{F_{1}}=\frac{1}{2}$, $M_{F_{2}}=-\frac{1}{2}$. The different magnetic field strengths where the resonances occur reflect the different Zeeman shifts experienced in the various states, i.e., states that resonate at $B_{1}$ have eigenvalues that are most easily perturbed to lower values by an external magnetic field. The resonances reported here have all been experimentally observed in [46] at the magnetic field strengths $B_{1 e}=159.14 \mathrm{G}, B_{2 e}=185.09 \mathrm{G}$, and $B_{3 e}=214.94 \mathrm{G}$. As previously emphasized, the calculated values are a result of an $a b$ inito calculation and the small

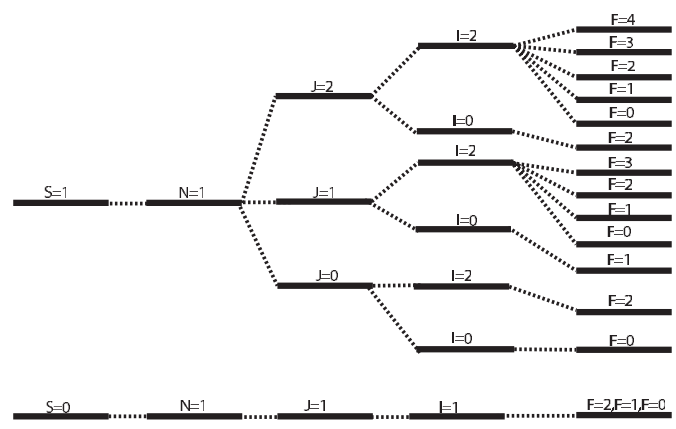

FIG. 7. Schematic diagram of the hyperfine structure in the $N$ $=1$ rotational level of ${ }^{6} \mathrm{Li}_{2}$. The Pauli exclusion principle has been taken into account. 


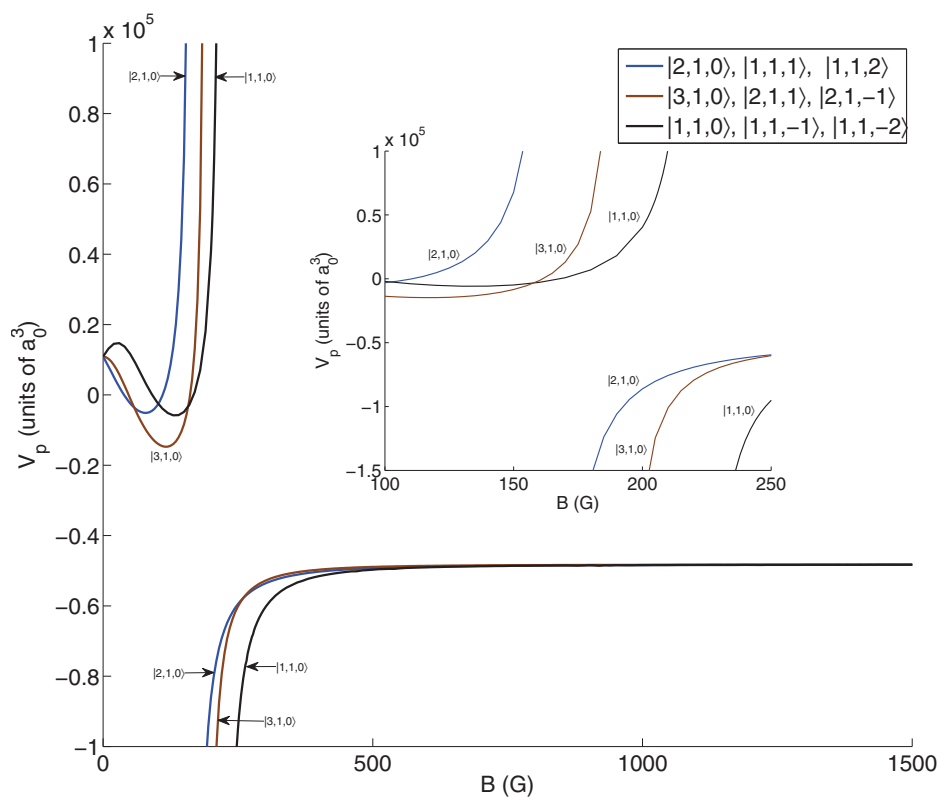

FIG. 8. (Color online) $p$-wave scattering volume as a function of magnetic field strength for all channels with $F_{1}=F_{2}=1 / 2$.

deviation in the resonance positions can be contributed to small errors in the singlet potential. All the $p$-wave resonances are found to be insensitive to the triplet potential.

Note that the relative positions of the calculated and experimentally observed resonances are in almost perfect agreement: $B_{2}-B_{1}=26.03 \mathrm{G}$ in the present $a b$ initio calculation whereas the experimental value is $25.95 \mathrm{G}$. Correspondingly, $\quad B_{3}-B_{2}=29.70 \mathrm{G} \quad a b$ initio and $B_{3}-B_{2}$ $=29.85 \mathrm{G}$ experimentally.

Whereas the $s$-wave resonances had a total nuclear spin of $I=0$ or $I=2$, the relevant nuclear spin for $p$-wave resonances is $I=1$. In analogy with the $s$-wave scattering lengths, the value of the $p$-wave scattering volume approaches the triplet value $V_{p}^{T}$ at high magnetic field strengths. The total electronic spin together with the total nuclear spin are both approximately good quantum numbers at high magnetic field strengths with the values 1 and 2, respectively.

The $p$-wave scattering volume as a function of magnetic field strength for states with $F_{1}=F_{2}=3 / 2$ (zero field) has a behavior which resembles that of the $s$-wave scattering length. Many states have $p$-wave scattering volumes that are approximately constant, whereas a few attains the triplet $p$-wave scattering volume in stronger fields.

TABLE III. Correspondence between the states $\left|n N M_{F}\right\rangle$ and the zero-field states $\left|F_{1} F_{2} F_{a} l F M_{F}\right\rangle . F_{1}, F_{2}, F_{a}$ and $F$ are good quantum numbers only at zero magnetic field. At zero field $F_{1}=F_{2}=\frac{1}{2}, F_{a}$ $=1$, and $N=1$ for all states.

\begin{tabular}{cccccc}
\hline \hline$\left|n N M_{F}\right\rangle$ & $\left|F M_{F}\right\rangle$ & $\left|n N M_{F}\right\rangle$ & $\left|F M_{F}\right\rangle$ & $\left|n N M_{F}\right\rangle$ & $\left|F M_{F}\right\rangle$ \\
\hline$|1,1,0\rangle$ & $|0,0\rangle$ & $|1,1,1\rangle$ & $|1,1\rangle$ & $|2,1,-1\rangle$ & $|2,-1\rangle$ \\
$|2,1,0\rangle$ & $|1,0\rangle$ & $|2,1,1\rangle$ & $|2,1\rangle$ & $|1,1,2\rangle$ & $|2,2\rangle$ \\
$|3,1,0\rangle$ & $|2,0\rangle$ & $|1,1,-1\rangle$ & $|1,-1\rangle$ & $|1,1,-2\rangle$ & $|2,-2\rangle$ \\
\hline \hline
\end{tabular}

\section{Short-range hyperfine- and spin-spin interaction}

\section{Hyperfine interaction}

The convenient representation of the hyperfine interaction in the Hund's case (a) basis easily allows for experimentation with the short-range $(r<20$ a.u. $)$ interaction. At $r$ $>20$ a.u. the hyperfine interaction is well described by the simpler atomic hyperfine Hamiltonian

$$
H_{\mathrm{hf}}=b_{f}\left(\mathbf{S}_{1} \cdot \mathbf{I}_{1}+\mathbf{S}_{2} \cdot \mathbf{I}_{2}\right)
$$

but this is not the case for $r<20$ a.u. as the electronic spin of atom 2 will start to couple to the nuclear spin of atom 1 and vice versa. In Sec. III A, it has been described how the hyperfine interaction is determined for all $r$ by the four molecular parameters $b_{f}(r), b_{f}^{\prime}(r), c(r)$, and $c^{\prime}(r)$. These hyperfine parameters were calculated as described in Sec. III B and are plotted in Fig. 10. For reference, the atomic values are indicated with dotted lines. We see from Fig. 10 that the calculated hyperfine parameters are rather similar to the atomic hyperfine parameters. As expected the difference is completely negligible for $r>20$ a.u., whereas there are only minor differences for 20 a.u. $>r>9$ a.u.; in fact the only difference is the $b_{f}^{\prime}$ parameter that slowly decreases, coupling the singlet and triplet states. For $r<9$ a.u., the molecular hyperfine parameters are rather different from the atomic values, hence the molecular hyperfine interactions deviates from the atomic interaction.

In scattering calculations, it is often assumed that the molecular hyperfine interaction at short range is equal to the atomic hyperfine interaction. It can be argued from Fig. 10 that one can safely use the atomic hyperfine interaction also at small distances for the present and similar systems without introducing significant errors, as the hyperfine parameters are 


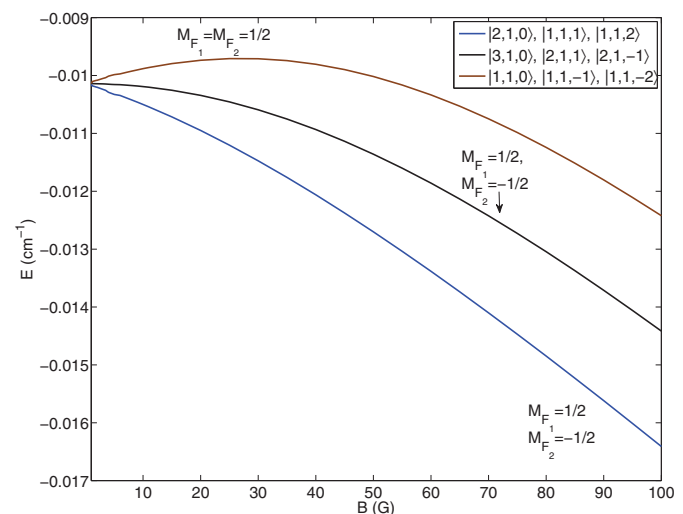

FIG. 9. (Color online) Energy vs magnetic field for states corresponding to the lowest hyperfine level.

only slowly varying. On the other hand, the molecular hyperfine parameters show significant deviations from the atomic values for small $r$.

We have tested the effect of the short-range molecular interaction by repeating some of the $s$-wave scattering calculations. The results obtained using the atomic hyperfine interaction have been compared with the results from a calculation including the complete $r$-dependent molecular hyperfine interaction. To investigate this effect, we have looked at the broad Feshbach resonance located at $B$ $\simeq 837 \mathrm{G}$ and calculated how much the resonance position is shifted upon changes in the short-range hyperfine interaction. As expected the resonance position is rather insensitive to the detailed short-range molecular hyperfine interaction. The shift is only $+0.02 \mathrm{G}$ when we replace the molecular interaction with the asymptotic atomic interaction. The much more narrow resonance at $B \simeq 545 \mathrm{G}$ is even less affected. Thus, we are led to conclude that the scattering length is quite insensitive to small changes in the hyperfine energy in the region of small $r$.

The strength of the short-range hyperfine interaction has been varied and the effect calculated. The hyperfine parameters were varied by multiplying the functions $b_{f}(r), c(r)$, $b_{f}^{\prime}(r)$, and $c^{\prime}(r)$ with a factor $m$ to yield a stronger or weaker short-range hyperfine interaction. The atomic hyperfine interaction valid at $r>20$ was left unchanged. Again we used the Feshbach resonance at $B \simeq 837 \mathrm{G}$ to test the reaction. The results are presented in Fig. 11, which shows that the shortrange hyperfine interaction needs a substantial increase to shift the resonance positions in a significant way. Finally, we also investigated the position of a $p$-wave Feshbach resonance and found the same insensitivity to changes in the short-range molecular hyperfine interaction.

It seems reasonable to expect that systems with similar electronic structures (i.e., other alkali molecules) have hyperfine interactions that are well described by the atomic interaction. This conclusion is valid with respect to cold collisions where the results are rather insensitive to small changes in the short-range potential. Diatomic molecules with several open-shell electrons that couple to the nuclear spin may have a much stronger short-range hyperfine interaction. Figure 11 shows that in such cases, the above conclusion does not hold and the short-range molecular hyperfine interaction becomes important also in cold collisions.
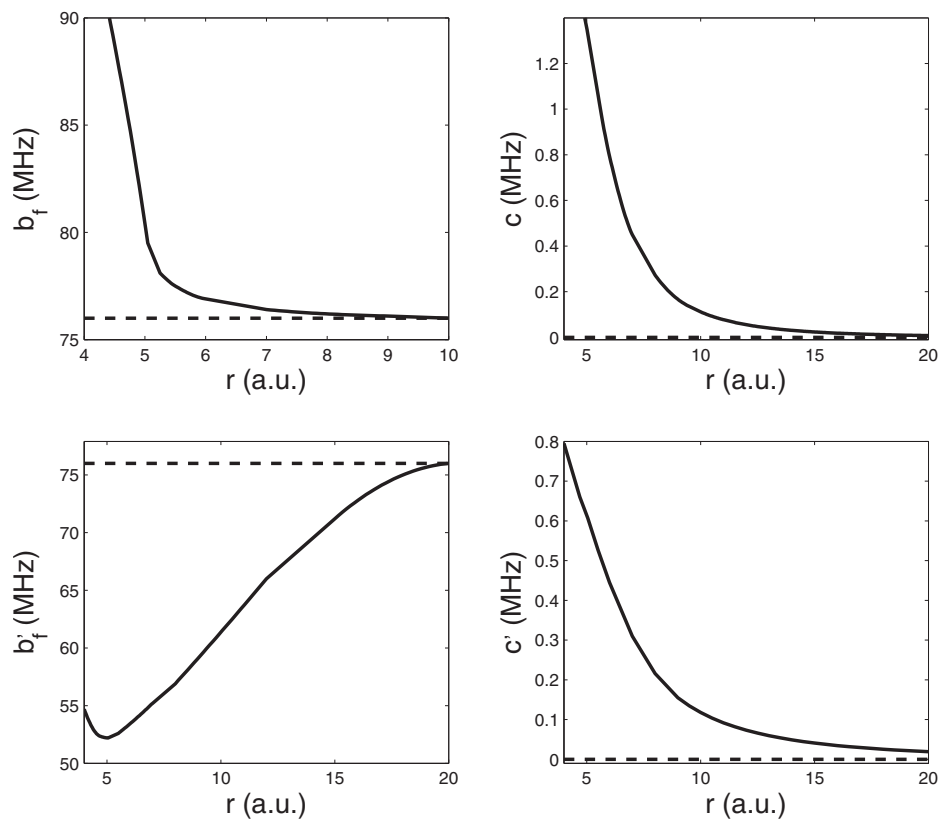

FIG. 10. Hyperfine parameters $b_{f}, c, c^{\prime}$ and $b_{f}^{\prime}$ as a function of $r$. See Sec. III B for a definition of the hyperfine parameters. Dotted lines show atomic hyperfine values included for comparison. 


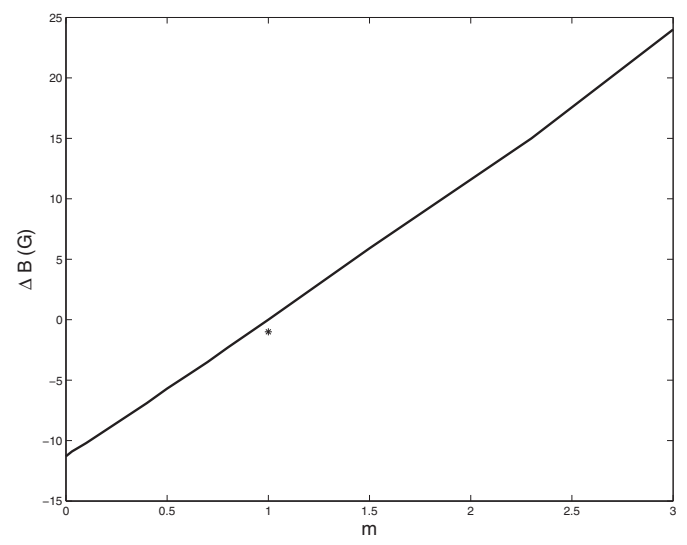

FIG. 11. Shift in resonance position vs strength of short-range hyperfine interaction. The hyperfine parameters are multiplied with a dimensionless factor $m$ to yield a stronger or weaker interaction keeping their original functional form. A stronger short-range hyperfine interaction changes the resonance position by tens of Gauss, whereas the integration in ${ }^{6} \mathrm{Li}_{2}$ is too weak relative to the atomic values. The star $(*)$ indicates resonance shift position using only the atomic hyperfine interaction for all $r$.

\section{Spin-spin interaction}

The spin-spin interaction was treated in Sec. III E. The spin-spin parameter $\lambda(r)$ has been calculated $a b$ initio in [52] for selected values of $r$ between 3.77 and 18.9 a.u. In $\mathrm{Li}_{2}$ the spin-spin parameter $\lambda(r)$ is very small, on the order of $-0.01 \mathrm{~cm}^{-1}$. We have, however, included the spin-spin interaction in the present calculations and investigated its effect on the various Feshbach resonances.

As might be expected, Feshbach resonances that are sensitive to the triplet interatomic potential are also very sensitive to the spin-spin interaction. For ${ }^{6} \mathrm{Li}_{2}$, this means that the Feshbach resonances at $B \simeq 837,814$, and $693 \mathrm{G}$ are sensitive to the spin-spin interaction, whereas the other ones are not.

To enable a more quantitative conclusion regarding the effect of the spin-spin interaction, the calculations were repeated for a series of different choices of $\lambda(r)$. In these repeated calculations, the function $\lambda(r)$ from [52] was multiplied by a factor $m$, with $m$ ranging from -5 to 5 . The results are presented in Fig. 12, which shows the results for three representative Feshbach resonances.

The three different Feshbach resonances investigated in Fig. 12 are representable for all the Feshbach resonances calculated. The three broad $s$-wave resonances behave similar to the $850 \mathrm{G}$ resonance, whereas the $p$-wave resonances behave similarly to the $p$-wave resonance in 12 . We have let the spin-spin parameter $\lambda(r)$ go to zero at $r \simeq 21$ a.u. as a natural continuation of the function $D(r)=2 \lambda(r)$ plotted in Fig. 3 in [52].

We conclude that Feshbach resonances in states sensitive to the triplet potential are affected by the spin-spin interaction. For these states, the spin-spin interaction is important

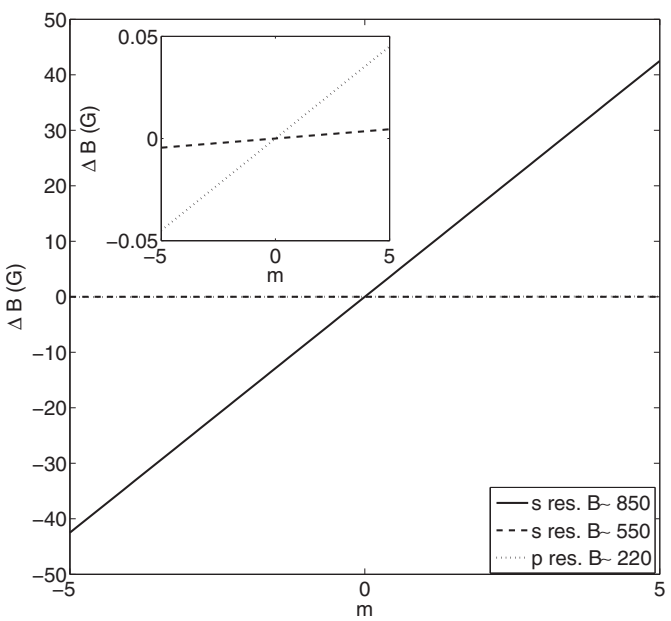

FIG. 12. Shift in resonance positions as a function of the spinspin interaction strength for three different Feshbach resonances. The dimensionless factor $m$ is multiplied with the spin-spin parameter $\lambda(r)$ to control the spin-spin interaction strength.

(see also Fig. 12). Feshbach resonances in states insensitive to the triplet potential are also insensitive to the spin-spin interaction as $S \simeq 0$ with $\Sigma=0$ in such states.

\section{SUMMARY}

We have used rather accurate $a b$ initio potentials available for ${ }^{6} \mathrm{Li}_{2}$ and solved the coupled equations to obtain $s$ - and $p$-waves scattering lengths for magnetic field strengths up to 1500 G. Hund's case (a) basis states were used to work out the molecular matrix elements required for the hyperfine, Zeeman, and rotational operators. Basis states which are in accordance with the Pauli principle upon the interchange of identical nuclei were constructed and used. Emphasis was put on including and working with the molecular hyperfine interaction in the short-range regime. However, it was found that the results of the scattering calculations were rather insensitive to the short-range hyperfine structure.

Several Feshbach resonances of both $s$ and $p$ types were found. The Feshbach resonance positions determined $a b$ initio deviated with plus 2-4 G from the experimentally measured positions available for comparison. By inspection, the deviations are found to be well represented by a constant shift. Using the $p$-wave resonance at $B=218.72 \mathrm{G}$ as a reference, one obtains $B_{\text {shift }}=B_{a b \text { initio }}-B_{\exp }=3.78$ G. Subtracting $B_{\text {shift }}$ from the calculated Feshbach resonance positions yields corrected positions $B_{\text {corrected }}$ which are in very good agreement with the experimental values. Table IV summarizes the discussion above. The constant shift is determined from a reference state which is sensitive to the singlet potential curve but insensitive to the triplet potential curve. Hence, $B_{\text {shift }}$ can be seen as a correction to the singlet potential. The broad $s$-wave resonances at $B=837,814$, and $693 \mathrm{G}$ are in addition sensitive to the triplet potential, but there is no ob- 
TABLE IV. Summary of calculated Feshbach resonances compared with experimentally observed ones. $\Delta B=B_{a b}$ initio $-B_{\text {exp }}$. See the text for a definition of $B_{\text {corrected }}$ and a further discussion of this quantity. The star ( *) indicates that there is no one-to-one correspondence between the state $\left|n N M_{F}\right\rangle$ and a unique free-atom state $\left|F_{1} M_{F_{1}} F_{2} M_{F_{2}}\right\rangle$.

\begin{tabular}{lccccc}
\hline \hline State $\left|n N M_{F}\right\rangle$ & $\left|F_{1} M_{F_{1}}\right\rangle\left|F_{2} M_{F_{2}}\right\rangle$ & $\begin{array}{c}B_{a b \text { initio }} \\
(\mathrm{G})\end{array}$ & $\begin{array}{c}B_{\text {exp }} \\
(\mathrm{G})\end{array}$ & $\begin{array}{c}\Delta B \\
(\mathrm{G})\end{array}$ & $\begin{array}{c}B_{\text {corrected }} \\
(\mathrm{G})\end{array}$ \\
\hline$|1,0,0\rangle$ & $\left|\frac{1}{2}, \frac{1}{2}\right\rangle\left|\frac{1}{2},-\frac{1}{2}\right\rangle$ & 837.13 & $822-834$ & $3.70-15.1$ & 833.35 \\
$|1,0,0\rangle$ & $\left|\frac{1}{2}, \frac{1}{2}\right\rangle\left|\frac{1}{2},-\frac{1}{2}\right\rangle$ & 544.90 & $543.28 \pm 0.08$ & $1.62 \pm 0.08$ & 541.12 \\
$|1,0,-1\rangle$ & $*$ & 692.89 & & & 689.11 \\
$|1,0,-2\rangle$ & $\left|\frac{3}{2},-\frac{3}{2}\right\rangle\left|\frac{1}{2},-\frac{1}{2}\right\rangle$ & 813.98 & & & 810.20 \\
$|1,1,0\rangle,|1,1,-1\rangle,|1,1,-2\rangle$ & $\left|\frac{1}{2},-\frac{1}{2}\right\rangle\left|\frac{1}{2},-\frac{1}{2}\right\rangle$ & 218.72 & $214.94 \pm 0.08$ & $3.78 \pm 0.08$ & 214.94 \\
$|2,1,0\rangle,|1,1,1\rangle,|1,1,2\rangle$ & $\left|\frac{1}{2}, \frac{1}{2}\right\rangle\left|\frac{1}{2}, \frac{1}{2}\right\rangle$ & 162.99 & $159.14 \pm 0.14$ & $3.85 \pm 0.14$ & 159.21 \\
$|3,1,0\rangle,|2,1,1\rangle,|2,1,-1\rangle$ & $\left|\frac{1}{2}, \frac{1}{2}\right\rangle\left|\frac{1}{2},-\frac{1}{2}\right\rangle$ & 189.02 & $185.09 \pm 0.08$ & $3.93 \pm 0.08$ & 185.24 \\
\hline \hline
\end{tabular}

vious way to obtain an additional correction for the triplet state that would affect only those particular resonances.

\section{APPENDIX: TRANSFORMATION BETWEEN $F F$-COUPLED STATES AND MOLECULAR HUND'S CASE}

\section{(A) STATES}

We start with the $F F$-coupled states $\left|q S_{1} M_{S_{1}} S_{2} M_{S_{2}}\right\rangle$ and specialize to alkali atoms where $L_{1}=L_{2}=0$. For this reason, $L_{1}$ and $L_{2}$ are suppressed in the notation. The $F F$-coupled states $\left|q S_{1} M_{S_{1}} S_{2} M_{S_{2}}\right\rangle$ have both a spatial part with collective quantum number $q$ and an electronic spin part. To obtain the connection with the Hund's case (a) states, we form the total electronic spin $\mathbf{S}=\mathbf{S}_{1}+\mathbf{S}_{2}$,

$$
\left|q S M_{S}\right\rangle=\sum_{M_{S_{1}, M_{S_{2}}}}\left\langle S_{1} M_{S_{1}} S_{2} M_{S_{2}} \mid S M_{S}\right\rangle\left|q S_{1} M_{S_{1}} S_{2} M_{S_{2}}\right\rangle .
$$

The states $\left|q S M_{S}\right\rangle$ are assumed to be eigenstates of the operators $I_{t}, I_{e}$ and $I_{n}$ (see Sec. II B). It follows from Eq. (A1) that the states $\left|q S_{1} M_{S_{1}} S_{2} M_{S_{2}}\right\rangle$ can be written as

$$
\left|q S_{1} M_{S_{1}} S_{2} M_{S_{2}}\right\rangle=\sum_{S M_{S}}\left\langle S_{1} M_{S_{1}} S_{2} M_{S_{2}} \mid S M_{S}\right\rangle\left|q S M_{S}\right\rangle,
$$

thus they are in general not eigenstates for the symmetry operators $I_{e}$ and $I_{n}$. At this point, it is appropriate to make a comment regarding the situation when $L_{i} \neq 0$. In such cases, the expression (A1) would still be valid although the replacement $\left|q S M_{S}\right\rangle \rightarrow\left|q L_{1} L_{2} M_{L_{1}} M_{L_{2}} S \Sigma\right\rangle$ is needed. To form the states $\left|q \Lambda S M_{S}\right\rangle$, we would then write

$$
\left|q \Lambda S M_{S}\right\rangle=\sum_{M_{L_{1}}+M_{L_{2}}=\Lambda} C_{M_{L_{1}}, M_{L_{2}}}\left|L_{1} L_{2} M_{L_{1}} M_{L_{2}} S M_{S}\right\rangle,
$$

where the coefficients $C_{M_{L_{1}}, M_{L_{2}}}$ are determined by the interaction between the atoms. In Eqs. (A1) and (A3) the quantum number $M_{S}$ refers to the space-fixed axis. The relation between space-fixed and molecule-fixed states is

$$
\left|q S M_{S}\right\rangle=\sum_{\Sigma} \mathcal{D}_{M_{S}, \Sigma}^{S}(\phi, \theta, 0)^{*}|q S \Sigma\rangle,
$$

where $\mathcal{D}$ is the rotation matrix [28]. We also need the diatomic rotational wave function [53],

$$
\Psi_{N M_{N} \Lambda}(\phi, \theta)=\left(\frac{2 N+1}{4 \pi}\right)^{1 / 2} \mathcal{D}_{M_{N}, \Lambda}^{N}(\phi, \theta, 0)^{*} .
$$

$N$ is the total molecular angular momentum excluding electronic and nuclear spin. When $L_{i}=0, N$ represents only the mechanical rotation. In addition, whenever $\Lambda=0$, the rotational wave function simplifies to the spherical harmonics $\Psi_{N M_{N} 0}(\phi, \theta)=Y_{N, M_{N}}(\theta, \phi)$.

Multiplying the states $\left|q S M_{S}\right\rangle$ and $\left|N M_{N} \Lambda\right\rangle$, using Eq. (A5) for the rotational wave function and contracting the two $\mathcal{D}$ matrices gives

$$
\begin{gathered}
\Psi_{q S M_{S} N M_{N} \Lambda}=\left(\frac{2 N+1}{4 \pi}\right)^{1 / 2} \sum_{\Sigma} \mathcal{D}_{M_{S}, \Sigma}^{S}(\phi, \theta, 0)^{*} \mathcal{D}_{M_{N}, \Lambda}^{N}(\phi, \theta, 0)^{*} \psi_{q S \Sigma}, \\
=\left(\frac{2 N+1}{4 \pi}\right)^{1 / 2} \sum_{\Sigma, J}(2 J+1)\left(\begin{array}{ccc}
S & N & J \\
M_{S} & M_{N} & -M_{J}
\end{array}\right)\left(\begin{array}{ccc}
S & N & J \\
\Sigma & \Lambda & -\Omega
\end{array}\right) \mathcal{D}_{-M_{J},-\Omega}^{J}(\phi, \theta, 0) \psi_{q S \Sigma}, \\
=\left(\frac{2 N+1}{4 \pi}\right)^{1 / 2}(-1)^{\Omega-M_{J}} \sum_{\Sigma, J}(2 J+1)\left(\begin{array}{ccc}
S & N & J \\
M_{S} & M_{N} & -M_{J}
\end{array}\right)\left(\begin{array}{ccc}
S & N & J \\
\Sigma & \Lambda & -\Omega
\end{array}\right) \mathcal{D}_{M_{J}, \Omega}^{J}(\phi, \theta, 0)^{*} \psi_{q S \Sigma} .
\end{gathered}
$$


At this point, we introduce the nuclear-spin states $\left|I_{1} M_{I_{1}}\right\rangle\left|I_{2} M_{I_{2}}\right\rangle$ and rotate them into the molecule-fixed coordinate system. The projections $M_{I_{1}}$ and $M_{I_{2}}$ are defined on the space-fixed axis. We have the relations

$$
\left|I_{i} M_{I_{i}}\right\rangle=\sum_{\Omega_{I_{i}}} \mathcal{D}_{M_{I_{i}}, \Omega_{I_{i}}}^{I_{i}}(\phi, \theta, 0)^{*}\left|I_{i} \Omega_{I_{i}}\right\rangle
$$

for $i=1,2$. Contracting the matrices $\mathcal{D}_{M_{I_{1}}, \Omega_{I_{1}}}^{I_{1}}(\phi, \theta, 0)^{*} \mathcal{D}_{M_{I_{2}}, \Omega_{I_{2}}}^{I_{2}}(\phi, \theta, 0)^{*} \quad\left(\mathbf{I}=\mathbf{I}_{1}+\mathbf{I}_{2}\right)$, and using $\mathcal{D}_{-M_{I}, \Omega_{I}}^{I}(\phi, \theta, 0)=($ $-1)^{\Omega_{I}-M_{I}} \mathcal{D}_{M_{I}, \Omega_{I}}^{I}(\phi, \theta, 0)^{*}$, we may write

$$
\begin{aligned}
& \Psi_{q N M_{N} \Lambda S \Sigma I_{1} M_{I_{1}} I_{2} M_{I_{2}}}=\left(\frac{2 N+1}{4 \pi}\right)^{1 / 2} \sum_{\Sigma, J, I,}(-1)^{\Omega-M_{J}+\Omega_{I}-M_{I}(2 J+1)(2 I+1)}\left(\begin{array}{ccc}
S & N & J \\
M_{S} & M_{N} & -M_{J}
\end{array}\right)\left(\begin{array}{ccc}
S & N & J \\
\Sigma & \Lambda & -\Omega
\end{array}\right)\left(\begin{array}{ccc}
I_{1} & I_{2} & I \\
M_{I_{1}} & M_{I_{2}} & -M_{I}
\end{array}\right) \\
& \Omega_{I_{1}}, \Omega_{I_{2}} \\
& \times\left(\begin{array}{ccc}
I_{1} & I_{2} & I \\
\Omega_{I_{1}} & \Omega_{I_{2}} & -\Omega_{I}
\end{array}\right) \mathcal{D}_{M_{I}, \Omega_{I}}^{I}(\phi, \theta, 0)^{*} \mathcal{D}_{M_{J}, \Omega}^{J}(\phi, \theta, 0)^{*} \psi_{q S \Sigma I_{1} \Omega_{I_{1}} I_{2} \Omega_{I_{2}}},
\end{aligned}
$$

or if we define $\mathbf{F}=\mathbf{J}+\mathbf{I}$ together with the total angular-momentum function [53]

$$
\Psi_{F M_{F} \Omega_{F}}(\phi, \theta)=\left(\frac{2 F+1}{4 \pi}\right)^{1 / 2} \mathcal{D}_{M_{F}, \Omega_{F}}^{F}(\phi, \theta, 0)^{*},
$$

this can be written as

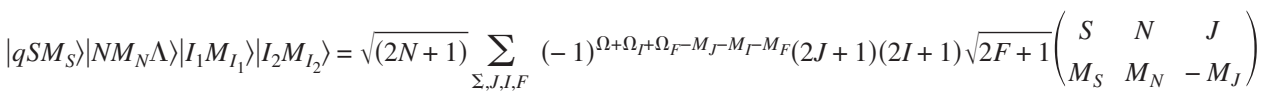

$$
\begin{aligned}
& \times\left(\begin{array}{ccc}
S & N & J \\
\Sigma & \Lambda & -\Omega
\end{array}\right)\left(\begin{array}{ccc}
I_{1} & I_{2} & I \\
M_{I_{1}} & M_{I_{2}} & -M_{I}
\end{array}\right)\left(\begin{array}{ccc}
I_{1} & I_{2} & I \\
\Omega_{I_{1}} & \Omega_{I_{2}} & -\Omega_{I}
\end{array}\right)\left(\begin{array}{ccc}
J & I & F \\
M_{J} & M_{I} & -M_{F}
\end{array}\right)\left(\begin{array}{ccc}
J & I & F \\
\Omega & \Omega_{I} & -\Omega_{F}
\end{array}\right) \\
& \times|q S \Sigma\rangle\left|I_{1} \Omega_{I_{1}}\right\rangle\left|I_{2} \Omega_{I_{2}}\right\rangle\left|F M_{F} \Omega_{F}\right\rangle .
\end{aligned}
$$

To establish Eq. (A10), we have contracted $\mathcal{D}_{M_{J}, \Omega_{J}}^{J}(\phi, \theta, 0)^{*}$ and $\mathcal{D}_{M_{I}, \Omega_{I}}^{I}(\phi, \theta, 0)^{*}$.

The $F F$-coupled basis states $\left|q F_{1} F_{2} F_{a} l F M_{F}\right\rangle$ are easily written in terms of the states $\left|q S_{1} M_{S_{1}} S_{2} M_{S_{2}}\right\rangle$ together with the nuclear-spin states $\left|I_{1} M_{I_{1}}\right\rangle\left|I_{2} M_{I_{2}}\right\rangle$, and after a piece of algebra one obtains the transformation to the $\left|q S M_{S}\right\rangle\left|N M_{N}\right\rangle\left|I_{1} M_{I_{1}}\right\rangle\left|I_{2} M_{I_{2}}\right\rangle$ basis,

$$
\begin{aligned}
\left|q F_{1} F_{2} F_{a} l F M_{F}\right\rangle= & \sum \sum_{\substack{M_{F_{a}}, m_{l}, M_{F_{1}}, M_{F_{2}}}} \sum_{M_{S_{1}}, M_{S_{2}}, M_{I_{2}}} \sum_{S, M_{S}}(-1)^{F_{a}-l+M_{F}+F_{1}-F_{2}+M_{F_{a}}+S_{1}-I_{1}+M_{F_{1}}+S_{2}-I_{2}+M_{F_{2}}+S_{1}-S_{2}+M_{S}} \\
& \times \sqrt{(2 F+1)\left(2 F_{a}+1\right)\left(2 F_{1}+1\right)\left(2 F_{2}+1\right)(2 S+1)}\left(\begin{array}{ccc}
l & F_{a} & F \\
m_{l} & M_{F_{a}} & -M_{F}
\end{array}\right)\left(\begin{array}{ccc}
F_{1} & F_{2} & F_{a} \\
M_{F_{1}} & M_{F_{2}} & -M_{F_{a}}
\end{array}\right) \\
& \times\left(\begin{array}{ccc}
S_{1} & I_{1} & F_{1} \\
M_{S_{1}} & M_{I_{1}} & -M_{F_{1}}
\end{array}\right)\left(\begin{array}{ccc}
S_{2} & I_{2} & F_{2} \\
M_{S_{2}} & M_{I_{2}} & -M_{F_{2}}
\end{array}\right)\left(\begin{array}{ccc}
S_{1} & S_{2} & S \\
M_{S_{1}} & M_{S_{2}} & -M_{S}
\end{array}\right)\left|q S M_{S}\right\rangle\left|N M_{N} \Lambda\right\rangle\left|I_{1} M_{I_{1}}\right\rangle\left|I_{2} M_{I_{2}}\right\rangle,
\end{aligned}
$$

an expression which is lengthy, but rather easy to derive. Combining Eqs. (A10) and (A11) yields the relevant transformation matrix elements $\left\langle q S \Sigma \Omega_{I_{1}} \Omega_{I_{2}} F M_{F} \Omega_{F} \mid q F_{1} F_{2} F_{a} l F M_{F}\right\rangle$ reported in Eq. (16).

[1] H. Feshbach, Ann. Phys. (N.Y.) 5, 357 (1958).

[2] B. DeMarco and D. S. Jin, Science 285, 1703 (1999).

[3] E. Tiesinga, B. J. Verhaar, and H. T. C. Stoof, Phys. Rev. A 47, 4114 (1993).
[4] E. P. Timmermans, P. Tommasini, M. Hussein, and A. Kerman, Phys. Rep. 315, 199 (1999).

[5] E. A. Donley, N. R. Claussen, S. T. Thompson, and C. E. Wieman, Nature (London) 417, 529 (2002). 
[6] J. Cubizolles, T. Bourdel, S. J. J. M. F. Kokkelmans, G. V. Shlyapnikov, and C. Salomon, Phys. Rev. Lett. 91, 240401 (2003).

[7] S. Jochim, M. Bartenstein, A. Altmeyer, G. Hendl, C. Chin, J. H. Denschlag, and R. Grimm, Phys. Rev. Lett. 91, 240402 (2003).

[8] C. A. Regal, M. Greiner, and D. S. Jin, Phys. Rev. Lett. 92, 083201 (2004).

[9] K. E. Strecker, G. B. Partridge, and R. G. Hulet, Phys. Rev. Lett. 91, 080406 (2003).

[10] S. Jochim, M. Bartenstein, A. Altmeyer, G. Hendl, S. Riedl, C. Chin, J. H. Denschlag, and R. Grimm, Science 302, 2101 (2003).

[11] M. Greiner, C. A. Regal, and D. S. Jin, Nature (London) 426, 537 (2003)

[12] M. W. Zwierlein, C. A. Stan, C. H. Schunck, S. M. F. Raupach, S. Gupta, Z. Hadzibabic, and W. Ketterle, Phys. Rev. Lett. 91, 250401 (2003).

[13] C. A. Regal, M. Greiner, and D. S. Jin, Phys. Rev. Lett. 92 , 040403 (2004).

[14] M. Bartenstein, A. Altmeyer, S. Riedl, S. Jochim, C. Chin, J. H. Denschlag, and R. Grimm, Phys. Rev. Lett. 92, 120401 (2004).

[15] H. Heiselberg, Phys. Rev. A 63, 043606 (2001).

[16] B. Gao, Phys. Rev. A 54, 2022 (1996).

[17] M. Abramowitz and I. S. Stegun, Handbook of Mathematical Functions (U.S. Natl. Bur. Stand, Washington, DC, 1964).

[18] B. R. Johnson, J. Comput. Phys. 13, 445 (1973).

[19] L. D. Landau and E. M. Lifshitz, Quantum Mechanics, 3rd ed. (Pergamon Press, Oxford, 1977), Chap. 11, p. 307.

[20] J. P. Burke, C. H. Greene, and J. L. Bohn, Phys. Rev. Lett. 81, 3355 (1998).

[21] B. Gao, E. Tiesinga, C. J. Williams, and P. S. Julienne, Phys. Rev. A 72, 042719 (2005).

[22] H. T. C. Stoof, J. M. V. A. Koelman, and B. J. Verhaar, Phys. Rev. B 38, 4688 (1988).

[23] G. Herzberg, Spectra of Diatomic Molecules (Van Nostrand, New York, 1950).

[24] L. D. Landau and E. M. Lifshitz, Quantum Mechanics, 3rd ed. (Butterworth-Heinemann, London, 1977), Chap. 11, p. 307.

[25] R. N. Zare, A. L. Schmeltekopf, W. J. Harrop, and D. L. Albritton, J. Mol. Spectrosc. 46, 37 (1973).

[26] V. P. Bellary, T. K. Balasubramanian, and B. J. Shetty, Pramana, J. Phys. 51, 445 (1998).

[27] E. Wigner and E. E. Witmer, Z. Phys. 51, 859 (1928).

[28] D. M. Brink and G. R. Satchler, Angular Momentum (Claren- don Press, Oxford, 1993), Chap. 5.

[29] M. Larsson, Phys. Scr. 23, 835 (1981).

[30] A. R. Edmonds, Angular Momentum in Quantum Mechanics, 2nd ed. (Princeton University Press, Princeton, NJ, 1960).

[31] P. Kristiansen and L. Veseth, J. Chem. Phys. 84, 2711 (1986).

[32] L. Veseth, J. Mol. Spectrosc. 63, 180 (1976).

[33] J. M. Brown, Mol. Phys. 31, 1517 (1976).

[34] S. P. McGlynn, T. Azumi, and M. Kinoshita, Molecular Spectroscopy of the Triplet State (Prentice-Hall, Englewood Cliffs, NJ, 1969).

[35] O. Vahtras, B. Minaev, O. Loboda, and H. Ågren, Chem. Phys. 279, 133 (2002).

[36] G. Audi, A. H. Wapstra, and C. Thibault, Nucl. Phys. A. 729, 337 (2003).

[37] A. A. Zavitsas, J. Mol. Spectrosc. 221, 67 (2003).

[38] B. Barakat, R. Bacis, E. Carrot, S. Churrassy, P. Crotez, and F. Martin, Chem. Phys. 102, 215 (1986).

[39] D. D. Konowalow and M. L. Olson, J. Chem. Phys. 71, 450 (1979).

[40] I. Schmidt-Mink, W. Müller, and W. Meyer, Chem. Phys. 92, 263 (1985).

[41] W. T. Zemke and W. C. Stwalley, J. Phys. Chem. 97, 2053 (1993).

[42] D. D. Konowalow, R. M. Regan, and M. E. Rosenkrantz, J. Chem. Phys. 81, 4534 (1984).

[43] L. D. Landau and E. M. Lifshitz, Quantum Mechanics, 3rd ed. (Pergamon Press, Oxford, 1977), p. 341.

[44] Z.-C. Yan, J. F. Babb, A. Dalgarno, and G. W. F. Drake, Phys. Rev. A 54, 2824 (1996).

[45] M. Marinescu, H. R. Sadeghpour, and A. Dalgarno, Phys. Rev. A 49, 982 (1994).

[46] C. H. Schunck, M. W. Zwierlein, C. A. Stan, S. M. F. Raupach, W. Ketterle, A. Simoni, E. Tiesinga, C. J. Williams, and P. S. Julienne, Phys. Rev. A 71, 045601 (2005).

[47] E. R. I. Abraham, W. I. McAlexander, J. M. Gerton, R. G. Hulet, R. Cote, and A. Dalgarno, Phys. Rev. A 55, R3299 (1997).

[48] M. Houbiers, H. T. C. Stoof, W. I. McAlexander, and R. G. Hulet, Phys. Rev. A 57, R1497 (1998).

[49] J. L. Bohn, Phys. Rev. A 61, 053409 (2000).

[50] H. Suno, B. D. Esry, and C. H. Greene, Phys. Rev. Lett. 90, 053202 (2003).

[51] S. Gautam and D. Angom, e-print arXiv:0802.1569.

[52] B. Minaev, Spectrochim. Acta, Part A 62, 790 (2005).

[53] D. M. Brink and G. R. Satchler, Angular Momentum (Clarendon Press, Oxford, 1993). 


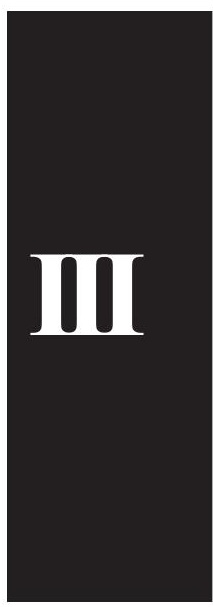





\title{
Feshbach resonances and transition rates for cold homonuclear collisions between ${ }^{39} \mathrm{~K}$ and ${ }^{41} \mathrm{~K}$ atoms
}

\author{
M. Lysebo and L. Veseth \\ Department of Physics, University of Oslo, N-0316 Oslo, Norway \\ (Received 2 November 2009; published 10 March 2010)
}

\begin{abstract}
We report results from close-coupling calculations for homonuclear ultracold collisions between potassium atoms, using the most up-to-date Born-Oppenheimer potential curves. The present study includes both of the bosonic isotopes ${ }^{39} \mathrm{~K}$ and ${ }^{41} \mathrm{~K}$. The $s$-wave scattering lengths as functions of the magnetic field strength for collisions between atoms in identical and different hyperfine states are obtained. Several Feshbach resonances are located and characterized for both isotopes. Comparison with experiments, where such data are available, show excellent agreement. We also study weakly bound molecular states of the $\mathrm{K}_{2}$ molecule in close relation to the calculated Feshbach resonances. Another objective of the present work is to study inelastic collisions in which the hyperfine states of the colliding atoms are changed. From this type of calculation we obtain transition rates as functions of the magnetic field strength. Finally, we discuss how such transition rates might be of importance for experimental work.
\end{abstract}

DOI: $10.1103 /$ PhysRevA.81.032702

PACS number(s): $34.50 . \mathrm{Cx}, 33.15 . \mathrm{Pw}, 37.10 . \mathrm{Mn}$

\section{INTRODUCTION}

Today ultracold atoms are routinely produced and subject to experimental studies in laboratories all around the world. Collisions and collision properties play an important role in most of these experiments. We focus on collisions between neutral atoms in the absence of light, with particular emphasis on the ultracold regime below $1 \mu \mathrm{K}$.

Collisions can be divided into three groups, elastic, inelastic, and reactive. In the present article we will only consider elastic and inelastic collisions, since reactive collisions are negligible at ultracold temperatures. Several methods for producing ultracold molecules rely on the properties of atomatom collisions and the ability to manipulate these properties with external fields. One example is buffer-gas cooling, which relies on repeated elastic collisions to transfer energy from the hot molecules to the cold buffer gas. This is a very effective and general method, although it has in the past not quite managed to cool below the milli-Kelvin temperature range. Recent results, however, show that this is possible [1]. Another example is cold dimer production through the method of photoassociation [2-5].

The present study is of most relevance to ultracold Feshbach molecules, which are formed via what is known as a Feshbach resonance. They are created when the energy of a bound diatomic vibrational state coincides with that of two free atoms, called a zero-energy resonance. This is possible in a magnetic field assuming that the energy of two free atoms and that of the bound molecular state can be tuned relative to each other by varying the magnetic field strength. When the energies of the bound molecular state and the two free atoms coincide, the phase shift induced by the interaction between the two atoms diverges and thereby so does the $s$-wave scattering length. This is recognized as a Feshbach resonance [6]. The ability to control the interatomic interaction in ultracold atomic gases is the fundamental reason why Feshbach resonances have become of such great importance. Feshbach resonances have been used to convert atomic gases into molecular Bose-Einstein condensates [7], to explore the BEC-BCS crossover [8], and to observe Efimov trimer states [9].
At low temperatures $T$, the de-Broglie wavelength, proportional to $T^{-1 / 2}$, is large. Only collisions with the very lowest value of the orbital angular momentum $l$ allow the atoms to get close enough to experience the interatomic potential; that is, the colliding atoms have no relative orbital angular momentum. In fact, the kinetic energy involved in ultracold atom-atom collisions is small enough to resolve even the hyperfine structure. In this regime the effect of the atom-atom interaction can be expressed in terms of the two-body scattering length $a_{s}$ [10]. This makes the $s$-wave scattering length an extremely important parameter in the ultracold regime.

In this article we investigate the stable bosonic isotopes of potassium, namely, ${ }^{39} \mathrm{~K}$ and ${ }^{41} \mathrm{~K}$. We study homonuclear atom-atom collisions between atoms in different hyperfine states. We use the most up-to-date, accurate interatomic potentials available and solve the full close-coupled equations. We calculate the $s$-wave scattering length as a function of the magnetic field and search for Feshbach resonances. In addition, we determine transition rates for $s$-wave atom-atom collisions for different hyperfine states and as a function of the magnetic field strength. The most obvious reason for studying these rates is to identify possible sources of trap loss. For magnetic trapping to work, the atoms have to be either in a low-field-seeking or a high-field-seeking state. Thus, there will be trap loss if a collision process induces a transition from a low-field-seeking state to a high-field-seeking state, or vice versa.

Feshbach resonances have been studied for most homonuclear alkali-metal species, although for the two potassium isotopes ${ }^{39} \mathrm{~K}$ and ${ }^{41} \mathrm{~K}$ there are at present few theoretical or experimental studies. However, some of the states that are subject to our calculations have been studied experimentally by D'Errico et al. [11]. In the case of the rather few theoretical predictions made in the past [11-13], we present more extensive calculations.

The study of Feshbach resonances in heteronuclear alkalimetal species is also a very active field, closely related to the topic of the present work. See, for example, the recent article by Gacesa et al. [14], and references therein. 
Finally, we remark that only a small portion of this article is devoted to the theory behind the model we have used in our computations. However, we emphasize that the present study basically uses the same approach to the coupled equations as previous studies. The interactions are, however, worked out in the molecular Hund's case (a) basis, which enables rather compact expressions for the matrix elements, along with the possibility to include the molecular short-range hyperfine interaction through calculation of only a few molecular hyperfine parameters. See [15] and references therein for an in-depth treatment of the model. The hyperfine energy levels of alkali-metal dimers has also been studied recently by Aldegunde et al. [16].

Throughout this article atomic units $\hbar=m_{e}=e=a_{0}=1$ are used, and we work within the Born-Oppenheimer approximation.

\section{THEORY}

A detailed description of the present model has been provided elsewhere [15]; thus, only a brief review is presented here. The Hamiltonian is written as

$$
\hat{H}=-\frac{1}{2 \mu} \nabla^{2}+\hat{H}_{\mathrm{ev}}+\hat{H}_{\mathrm{hf}}+\hat{H}_{\mathrm{Z}}
$$

with $\hat{H}_{\mathrm{ev}}$ as the electronic and vibrational Hamiltonian, $\hat{H}_{\mathrm{hf}}$ as the hyperfine Hamiltonian, and $\hat{H}_{\mathrm{Z}}$ as the Zeeman Hamiltonian. The first term in Eq. (1) represents the kinetic energy for the reduced mass $\mu$. The time-independent Schrödinger equation must be solved to obtain the wave function $\Psi(\mathbf{r}, \mathbf{x})$,

$$
\hat{H} \Psi(\mathbf{r}, \mathbf{x})=E \Psi(\mathbf{r}, \mathbf{x}),
$$

where $\mathbf{r}=(r, \theta, \phi), r$ is the internuclear separation, and $\theta$ and $\phi$ specify the orientation of the molecular axis in a space-fixed system. The electronic coordinates are collectively denoted $\mathbf{x}=\left[\mathbf{x}_{1}, \mathbf{x}_{2}, \ldots, \mathbf{x}_{n}\right]$. In the present context $E>0$, although very small due to the low temperature. Furthermore, $\Psi(\mathbf{r}, \mathbf{x})$ is expanded in the electronic functions $\phi_{b}(\mathbf{r}, \mathbf{x})$ as

$$
\Psi(\mathbf{r}, \mathbf{x})=\sum_{a} \frac{\psi_{a}(r)}{r} \phi_{a}(\mathbf{r}, \mathbf{x}),
$$

where $\psi_{a}(r)$ represents the relative motion of the collision partners. The functions $\phi_{a}(\mathbf{r}, \mathbf{x})$ are eigenfunctions for the relative orbital angular momentum operator. Using Eq. (3) and integrating over the electronic coordinates $\mathbf{x}=\left[\mathbf{x}_{1}, \mathbf{x}_{2}, \ldots, \mathbf{x}_{N}\right]$, the Schrödinger equation can be written as

$$
\begin{aligned}
& -\frac{1}{2 \mu} \frac{d^{2}}{d r^{2}} \psi_{a}(r)+\frac{l(l+1)}{2 \mu r^{2}} \psi_{a}(r) \\
& \quad+\sum_{b}\left\{V_{a b}^{\mathrm{ev}}+V_{a b}^{\mathrm{hf}}(r) \psi_{b}(r)+V_{a b}^{\mathrm{Z}}\right\} \psi_{b}(r)=E \psi_{a}(r) .
\end{aligned}
$$

In Eq. (4) the matrix elements $V_{a b}^{\mathrm{ev}}, V_{a b}^{\mathrm{hf}}$ and $V_{a b}^{\mathrm{Z}}$ are defined as

$$
\begin{gathered}
V_{a b}^{\mathrm{ev}}(r)=\left\langle\phi_{a}(\mathbf{r}, \mathbf{x})\left|\hat{H}_{\mathrm{ev}}\right| \phi_{b}(\mathbf{r}, \mathbf{x})\right\rangle, \\
V_{a b}^{\mathrm{hf}}(r)=\left\langle\phi_{a}(\mathbf{r}, \mathbf{x})\left|\hat{H}_{\mathrm{hf}}\right| \phi_{b}(\mathbf{r}, \mathbf{x})\right\rangle, \\
V_{a b}^{\mathrm{Z}}=\left\langle\phi_{a}(\mathbf{r}, \mathbf{x})\left|\hat{H}_{\mathrm{Z}}\right| \phi_{b}(\mathbf{r}, \mathbf{x})\right\rangle,
\end{gathered}
$$

and the nonadiabatic coupling terms arising from the kinetic energy terms have been neglected. The boundary conditions which are enforced on the wave function $\psi_{a}$ are $\psi_{a}(0)=0$ and

$$
\psi_{a}(r) \underset{r \rightarrow \infty}{\longrightarrow} \sum_{b} \phi_{b}(\mathbf{r}, \mathbf{x}) \frac{1}{\sqrt{k_{b}}}\left[j_{b}\left(k_{b} r\right) \delta_{a b}+g_{b}\left(k_{b} r\right) K_{b a}\right]
$$

The matrix $K$ is determined from the calculated solution $\psi_{a}(r)$ together with Eq. (8). In Eq. (8) $j_{l}(x)$ and $g_{l}(x)$ are the RiccatiBessel functions [17] for $E>E_{b}$ (i.e., open channels) and

$$
k_{b}=\sqrt{2 \mu\left|E-E_{b}\right|} \text {. }
$$

However, $j_{l}(x)$ and $g_{l}(x)$ must be replaced with the modified spherical Bessel functions [17] of first and third kind multiplied with $k_{b} \sqrt{r}$ whenever $E<E_{b}$. A channel state represents the quantum state of the system long before or long after the collision. The coupling between the open and the closed channels is very important for the existence of Feshbach resonances. This coupling is due to the hyperfine interaction for the present homonuclear system.

The matrix elements in Eqs. (5)-(7) are worked out in a molecular Hund's case (b) basis set and are given in detail in [15], together with a description of the molecular Hund's case (b) basis and its properties. Again we refer to [15] for further details. The channel states are constructed to respect the Pauli principle under the exchange of identical nuclei; see [18] for a detailed treatment.

Furthermore, the scattering matrix $S$ can be calculated as

$$
S=\frac{1+i K}{1-i K}
$$

once the $K$ matrix defined in Eq. (8) is known. The elements of the $S$ matrix are the scattering amplitudes. The $s$-wave scattering length $a_{s}$ is related to the diagonal elements of the $S$ matrix as

$$
S_{a a} \rightarrow e^{-2 i k_{a} a_{s}},
$$

when $k_{a} \rightarrow 0$. Once the $S$ matrix is obtained, the off-diagonal entries can be used to calculate the transition rate $G_{a \rightarrow b}$ as [19]

$$
G_{a \rightarrow b}=\lim _{k_{a} \rightarrow 0} \frac{\pi \hbar}{\mu k_{a}}\left|S_{a b}-\delta_{a b}\right|^{2},
$$

where $G_{a \rightarrow b}$ is measured in $\mathrm{m}^{3} / \mathrm{s}$. The expression for $G_{a \rightarrow b}$ is valid if the thermal energy is much smaller than the hyperfine and Zeeman energies. The rate $G_{a \rightarrow b}$ gives information about the amount of decay from channel $\left|\psi_{a}\right\rangle$ to a different channel $\left|\psi_{b}\right\rangle$ during a two-body collision. Thus, the rates can be used to predict the population of hyperfine state in a gas of trapped atoms or molecules. The transition rate $G_{a \rightarrow b}$ depends on the magnetic field strength $B$ through the matrix element $S_{a b}$.

The treatment of the hyperfine interaction warrants extra attention; see [15] and references therein. For diatomic homonuclear alkali-metal molecules, the coupling between the ${ }^{3} \Sigma_{u}^{+}-{ }^{1} \Sigma_{g}^{+}$states is due to this interaction. The molecular hyperfine interaction can be described by the four parameters $b_{f}, c, b_{f}^{\prime}$, and $c^{\prime}$, where $b_{f}$ is the Fermi-contact hyperfine interaction and $c$ is a parameter associated with the anisotropic part of the hyperfine Hamiltonian. The primed parameters $b_{f}^{\prime}$ and $c^{\prime}$ have a similar definition, but refer to the part of the interaction that is off-diagonal in the total molecular electronic 
TABLE I. Correspondence between the channel states $\left|n M_{F}\right\rangle$ and the two-atom states $\left|F_{1} M_{F_{1}} F_{2} M_{F_{2}}\right\rangle$. At $B=0$, the channel states and the two-atom states are identical. The orbital angular momentum quantum number is zero for all tabulated states. The two atoms are identical, and the two-atom states are symmetrized to respect the Pauli principle; that is, they are generally not simple product states of the type $\left|F_{1} M_{F_{1}}\right\rangle\left|F_{2} M_{F_{2}}\right\rangle$.

\begin{tabular}{ll|ll|ll}
\hline \hline$\left|n M_{F}\right\rangle$ & $\left|F_{1} M_{F_{1}} F_{2} M_{F_{2}}\right\rangle$ & $\left|n M_{F}\right\rangle$ & $\left|F_{1} M_{F_{1}} F_{2} M_{F_{2}}\right\rangle$ & $\left|n M_{F}\right\rangle$ & $\left|F_{1} M_{F_{1}} F_{2} M_{F_{2}}\right\rangle$ \\
\hline$|0, \pm 2\rangle$ & $|1, \pm 1,1, \pm 1\rangle$ & $|0, \pm 1\rangle$ & $|1, \pm 1,1,0\rangle$ & $|0,0\rangle$ & $|1,0,1,0\rangle$ \\
$|1,-2\rangle$ & $|1,0,2,-2\rangle$ & $|1,-1\rangle$ & $|1,1,2,-2\rangle$ & $|1,0\rangle$ & $|1,-1,1,1\rangle$ \\
$|1,2\rangle$ & $|1,1,2,1\rangle$ & $|1,1\rangle$ & $|1,1,2,0\rangle$ & $|2,0\rangle$ & $|1,1,2,-1\rangle$ \\
$|2,-2\rangle$ & $|1,-1,2,-1\rangle$ & $|2, \pm 1\rangle$ & $|1,0,2, \pm 11\rangle$ & $|3,0\rangle$ & $|1,0,2,0\rangle$ \\
$|2,2\rangle$ & $|1,0,2,2\rangle$ & $|3,-1\rangle$ & $|1,-1,2,0\rangle$ & $|4,0\rangle$ & $|1,-1,2,1\rangle$ \\
$|3, \pm 2\rangle$ & $|2,0,2, \pm 2\rangle$ & $|3,1\rangle$ & $|1,-1,2,2\rangle$ & $|5,0\rangle$ & $|2,-2,2,2\rangle$ \\
$|4, \pm 2\rangle$ & $|2, \pm 1,2, \pm 1\rangle$ & $|4, \pm 1\rangle$ & $|2, \pm 2,2, \mp 1\rangle$ & $|6,0\rangle$ & $|2,-1,2,1\rangle$ \\
& & $|5, \pm 1\rangle$ & $|2, \pm 1,2,0\rangle$ & $|7,0\rangle$ & $|2,0,2,0\rangle$ \\
\hline \hline
\end{tabular}

spin $S$. At long range the molecular hyperfine interaction simplifies and correlates with the atomic hyperfine splitting. In this limit, $c=c^{\prime}=0$ and $b_{f}$ as well as $b_{f}^{\prime}$ can be determined from the atomic hyperfine splitting.

For the present calculations the long-range description of the hyperfine interaction will be sufficient. The inclusion of the full molecular hyperfine interaction will influence the results in a rather insignificant way, as was shown and discussed in [15].

The standard Hund's case (b) states may in some sense represent the most convenient coupling model for a diatomic molecule. However, in the present case the interaction between the triplet and the singlet states render the total electronic spin useless as a good quantum number. The good quantum numbers are then the total atomic angular momenta $F_{1}$ and $F_{2}$ (for atom 1 and atom 2, respectively) and their projections $M_{F_{1}}$ and $M_{F_{2}}$, along with the rotational quantum number $l$ and the projection $M_{F}=M_{F_{1}}+M_{F_{2}}+M_{l}$. However, in a magnetic field only $l$ and $M_{F}$ remain good quantum numbers. Thus, the channels are written $\left|n, l, M_{F}\right\rangle$, where $n$ is introduced to distinguish between channels with equal $l$ and $M_{F}$ quantum numbers. The channels are assigned labels $n$ according to their energies, where the channel with the lowest energy is labeled $n=0$. We will be concerned with $s$-wave channel states, which means $l=0$, and we will omit $l$ when specifying the channels; thus, $\left|n, 0, M_{F}\right\rangle=\left|n, M_{F}\right\rangle$. The channel states are eigenfunctions for the complete Hamiltonian in the limit $r \rightarrow \infty$, and they are constructed to respect the Pauli exclusion principle upon interchange of nuclei and electrons. Table I shows the correspondence between the channel states and the two-atom hyperfine states.

\section{SINGLET AND TRIPLET INTERACTION POTENTIAL CURVES}

Calculations of properties associated with ultracold collisions between two atoms require reliable interatomic Born-Oppenheimer potential curves. Formally, the BornOppenheimer potential curve $E_{a}(r)$ for the electronic state $a$ can be obtained by solving the time-independent Schrödinger equation

$$
\hat{H}_{\mathrm{ev}} \phi_{a}(\mathbf{r}, \mathbf{x})=E_{a}(r) \phi_{a}(\mathbf{r}, \mathbf{x}),
$$

where $\hat{H}_{\mathrm{ev}}$ is the electronic Hamiltonian including the kinetic energy for all electrons and the Coulomb potential for
$N$ electrons and the two nuclei. Again $\mathbf{r}$ is the internuclear vector and $\mathbf{x}=\left[\mathbf{x}_{1}, \mathbf{x}_{2}, \ldots, \mathbf{x}_{N}\right]$ contains the electronic coordinates. One should be aware that ab initio calculations of highly accurate Born-Oppenheimer potentials represent a very challenging many-body problem. We will therefore make use of available interatomic potentials recently obtained from molecular spectroscopy by Falke et al. [20].

The ground states of the two potassium atoms have electronic spin $S_{1}=S_{2}=\frac{1}{2}$, and angular momentum $L_{1}=$ $L_{2}=0$; that is, the atomic ground state is ${ }^{2} S$.

The possible molecular electronic states are then the singlet state ${ }^{1} \Sigma_{g}^{+}$and the triplet state ${ }^{3} \Sigma_{u}^{+}[21,22]$ (see Fig. 1). At long range the molecular potentials can be written as a sum of dispersion terms and an exchange type of potential which diminishes exponentially,

$$
V(r)=-\frac{C_{6}}{r^{6}}-\frac{C_{8}}{r^{8}}-\frac{C_{10}}{r^{10}} \mp A r^{\gamma} e^{-\beta r},
$$

where the - sign applies to the ${ }^{1} \Sigma_{g}^{+}$state, and the + sign to the ${ }^{3} \Sigma_{u}^{+}$state. The constants $C_{6}, C_{8}, C_{10}, A, \gamma$, and $\beta$ are given in [20]. We have used the same interatomic potential for both isotopes of $\mathrm{K}_{2}$, thereby assuming the validity of the Born-Oppenheimer approximation.

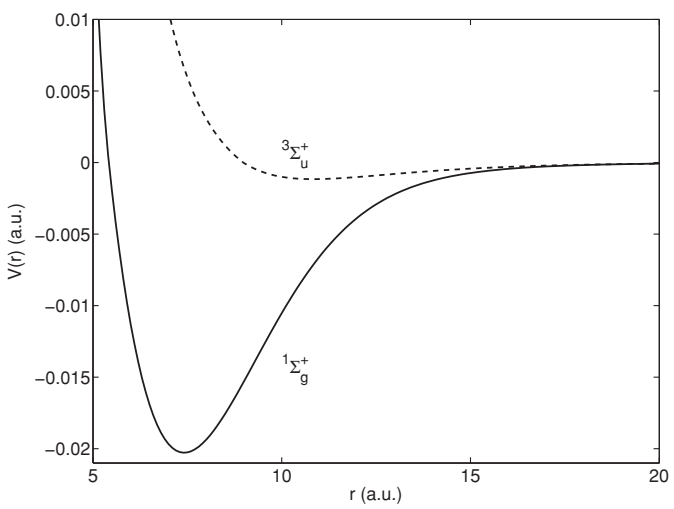

FIG. 1. Interatomic potential curves for the electronic states ${ }^{1} \Sigma_{g}^{+}$ (solid line) and ${ }^{3} \Sigma_{u}^{+}$(dotted line) in $\mathrm{K}_{2}$. 


\section{COMPUTATIONAL DETAILS}

The (improved [23]) log-derivative method of Johnson [24] was implemented in the $\mathrm{C}++$ programming language to obtain solutions to the set of coupled differential equations in Eq. (4). The matrix elements in Eqs. (5)-(7) were worked out in [15] and the expressions presented there have formed the basis for the present calculations. To give a complete representation of the relevant interactions in a system, one needs to include many more states than the few we want to study. The proper states to include can be found by studying how the interactions couple states with different quantum numbers. The Pauli principle is taken into account to ensure that we only include states that are allowed to exist in nature.

Included in the code is also the possibility to change between different basis states. These are the scattering channels, the two-atom basis and different molecular Hund's cases. The actual interactions are represented in the Hund's case (b) basis set. In the following sections we will occasionally include the quantum numbers for the total electronic spin $S$ and the total nuclear spin $I$ for the different channel states. Information regarding these quantum numbers are obtained by transforming from a basis where the channel states are defined to a suitable molecular Hund's case basis.

For the present system there is no need to perform interpolation in the singlet and triplet potential curves, as Falke et al. [20] provides an analytical function which is adequate for all values of $r$.

The atomic hyperfine splitting $\Delta$ between the two hyperfine levels with quantum numbers $F=2$ and $F=1$ for the ${ }^{39} \mathrm{~K}$ isotope is taken to be $\Delta=461.719720 \mathrm{MHz}$, a value obtained from [25]. Correspondingly, the splitting between the levels $F=2$ and $F=1$ is $254.013870 \mathrm{MHz}$ for ${ }^{41} \mathrm{~K}$, obtained from the same reference. Based on these measurements we calculate the molecular hyperfine parameters $b_{f}$ and $b_{f}^{\prime}$ (see [15] for more details regarding the molecular hyperfine parameters) as $b_{f}=\frac{\Delta}{4}$ and $b_{f}^{\prime}=-b_{f}$. Furthermore, the reduced mass for the ${ }^{39} \mathrm{~K}_{2}$ system is taken to be 35513.24612 a.u., obtained from the isotope mass reported in [26]. Similarly, the reduced mass for the ${ }^{41} \mathrm{~K}_{2}$ system is taken to be $37334.4202 \mathrm{a}$.u.

The scattering lengths for two spinless atoms interacting via the singlet potential curve are $138.80 a_{0}$ and $85.41 a_{0}$ for ${ }^{39} \mathrm{~K}$ and ${ }^{41} \mathrm{~K}$, respectively [20]. For the triplet potential, the scattering lengths are $-33.41 a_{0}$ and $60.27 a_{0}$ for ${ }^{39} \mathrm{~K}$ and ${ }^{41} \mathrm{~K}$, respectively [20]. These numbers are determined solely from the Born-Oppenheimer potentials. In the following sections we will refer to these values as the singlet and triplet background scattering lengths for the relevant isotope.

\section{RESULTS}

\section{A. Feshbach resonances and $s$-wave scattering lengths for the ${ }^{39} K_{2}$ system}

With the model outlined in Sec. II and in Ref. [15], together with the interatomic potentials described in Sec. IV, we have calculated $s$-wave scattering lengths for two colliding ${ }^{39} \mathrm{~K}$ atoms. The ${ }^{39} \mathrm{~K}$ isotope has a nuclear spin $I=\frac{3}{2}$ and one unpaired electron; hence, there are two possible quantized values for the total atomic angular momentum, that is, $F=2$ and $F=1$. With the two atoms infinitely separated, this system

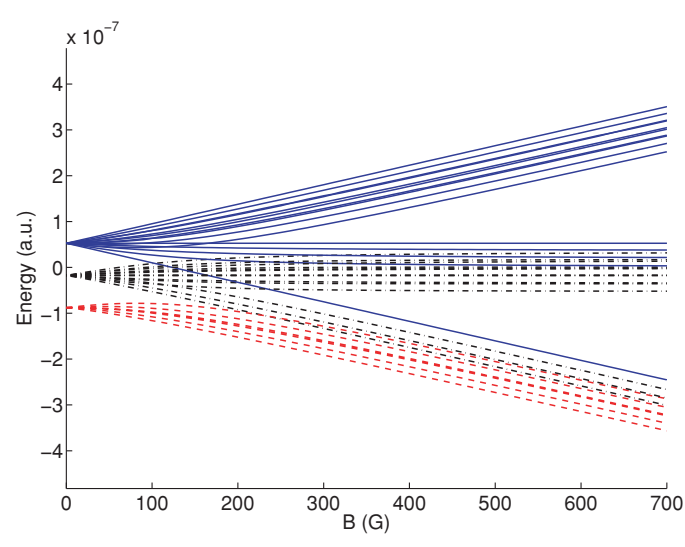

FIG. 2. (Color online) Energy as a function of magnetic field strength for all channels corresponding to $l=0$ in ${ }^{39} \mathrm{~K}_{2}$. At zero field only the three different hyperfine channels are resolved (blue solid lines, $F_{1}=F_{2}=2$; black dash-dot lines, $F_{1}=1, F_{2}=2$; red dashed lines, $F_{1}=F_{2}=1$ ). For $B>100 \mathrm{G}$, it is evident that $F_{1}$ and $F_{2}$ are no longer good quantum numbers.

has three degenerate energy levels, assuming zero magnetic field and no relative orbital angular momentum. The energy depends on the atomic hyperfine state specified by the quantum numbers $F_{1}$ and $F_{2}$, which denote the total atomic angular momentum for atoms 1 and 2, respectively. The three possible combinations of $F_{1}$ and $F_{2}$ are $F_{1}=F_{2}=1, F_{1}=F_{2}=2$ and $F_{1}=1, F_{2}=2$ (same as $\left.F_{1}=2, F_{1}=1\right)$, which means that there are three hyperfine manifolds (see also Fig. 2).

The channel states $\left|n, M_{F}\right\rangle$ respond differently to the magnetic field which affects the system through the Zeeman interaction. Figure 2 shows the energy as a function of the magnetic field strength for all channels (corresponding to the states $\left|F_{1} M_{F_{1}} F_{2} M_{F_{2}}\right\rangle$ at zero magnetic field; see Table I). Ten channels have large negative Zeeman shifts and are possible candidates for Feshbach resonances. Six of these channels correspond to the lowest hyperfine manifold $F_{1}=F_{2}=1$ at zero magnetic field. In addition, the three channel states $\left(\left|n, M_{F}\right\rangle\right)|1,-1\rangle,|1,-2\rangle$, and $|0,-3\rangle$ from the $F_{1}=1, F_{2}=$ 2 manifold and the $|0,-4\rangle$ channel from the $F_{1}=F_{2}=2$ hyperfine manifold also have large negative Zeeman shifts. All relevant channels for the present discussion are defined in Fig. 3 and Table I.

We will now consider collisions between two atoms in the lowest atomic hyperfine state with $F_{1}=F_{2}=1$. These atomic states are not susceptible to spin relaxation and may therefore be most convenient for experimental work. Figure 4 shows the $s$-wave scattering lengths $a_{s}$ as a function of the magnetic field strength $B$.

The most prominent feature seen in Fig. 4 is a number of Feshbach resonances, that is, the divergences in the scattering length $a_{s}(B)$. The number of resonances and the corresponding field strength vary from channel to channel.

Channels with the quantum numbers $M_{F}= \pm 2$ and $l=0$, originating from the lowest hyperfine manifold, represent collisions between atoms in the two atomic hyperfine states $\left|F_{1} M_{F_{1}}\right\rangle=|1, \pm 1\rangle_{A}$ and $\left|F_{2} M_{F_{2}}\right\rangle=|1, \pm 1\rangle_{A}$. To avoid any 

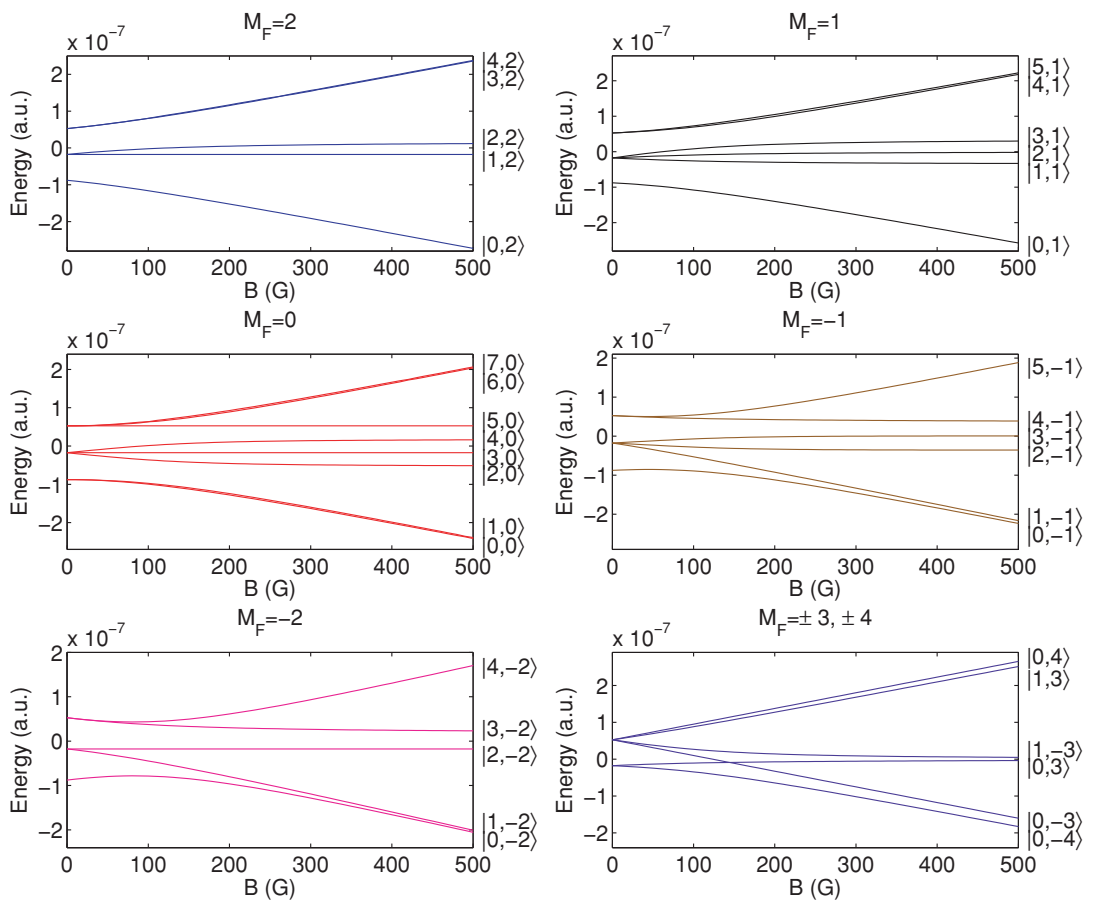

FIG. 3. (Color online) Energy vs magnetic field defining the channel states $\left|n, M_{F}\right\rangle$, where $M_{F}=M_{F_{1}}+M_{F_{2}}$ and $n$ is used as an additional label to distinguish channels with the same value of $M_{F}$. The channel with the lowest energy is labeled $n=0$. Only channels corresponding to zero orbital angular momentum are shown and the magnetic field strength varies between 0 and $500 \mathrm{G}$ (cf. Fig. 2).

possible confusion, we will use the subscript $A$ for atomic states, whereas no subscript will be used for scattering channels. In the right panel of Fig. $4\left(M_{F}= \pm 2\right)$ we see the characteristic features of six Feshbach resonances, three in the $|0,-2\rangle$ channel and three in the $|0,2\rangle$ channel. Away from all resonances $a_{s}(B)$ attains moderate negative values $\simeq-35 a_{0}$. The Feshbach resonances occur at different magnetic field strengths for the $|0,2\rangle$ and $|0,-2\rangle$ channels, as they respond differently to the magnetic field. More detailed information about each resonance is given in Table II. We note that there are three wide resonances in the $|0,-2\rangle$ channel and also one in the $|0,2\rangle$ channel. The remaining two resonances, which both occur in the $|0,2\rangle$ channel, are narrow with a width below $1 \mathrm{G}$. Experimentally, the wide resonances are the most favorable and the resonances occurring in the $M_{F}= \pm 2$ channels are experimentally the best studied Feshbach resonances for this isotope [11]. All available experimental data have been included for comparison in Table II. The comparison shows excellent agreement.

In the top left panel of Fig. 4 we report the $s$-wave scattering lengths for channels with $M_{F}= \pm 1$. Three Feshbach resonances are found in both channels $|0,1\rangle$ and $|0,-1\rangle$. The $|0, \pm 1\rangle$ channels correspond to collisions between atoms in the atomic hyperfine states $|1,0\rangle_{A}$ and $|1, \pm 1\rangle_{A}$. Note that these collisions are between atoms in different hyperfine states. One broad and two narrow resonances are found in the $|0,1\rangle$ channel. The broad resonance occurs at $B \simeq 450 \mathrm{G}$ and is about $40 \mathrm{G}$ wide. Two broad resonances are also found in the $|0,-1\rangle$ channel, at $B \simeq 100 \mathrm{G}$ and at $B \simeq 500 \mathrm{G}$, together with a narrow resonance at $B \simeq 720 \mathrm{G}$. Far away from all resonance, $a_{s}(B)$ attains a value close to $-35 a_{0}$.

Finally, in the bottom left panel of Fig. 4 we have plotted the $s$-wave scattering lengths $a_{S}(B)$ for collisions between atoms in the identical atomic hyperfine state $|1,0\rangle_{A}$ (dashed blue line), and for collisions between atoms in the different hyperfine states $|1,-1\rangle_{A}$ and $|1,1\rangle_{A}$ (Recall that the subscript $A$ is used to denote atomic states). The functions $a_{s}(B)$ are seen to vary rapidly at the relevant magnetic field strengths $B$. This is in particular true for the $|0,0\rangle$ channel which exhibits six Feshbach resonances, two broad (at $B=470$ and 490 $\mathrm{G}$ ) and four narrow (at $B=58,65.6,825.0$, and $832.3 \mathrm{G}$ ) [cf. Fig. (4)].

The results presented here show that the resonance widths varies from 100 to $0.10 \mathrm{G}$ for the present isotope. The width depends on the hyperfine interaction strength.

Tiesinga et al. [27] found that the scattering length as a function of the magnetic field strength $B$ could be expressed as

$$
a_{s}(B)=a_{\mathrm{bg}}\left(1-\frac{\Delta}{B-B_{0}}\right),
$$

close to a Feshbach resonance. In Eq. (15) the symbol $\Delta$ represents the width of a given resonance, $B_{0}$ is the field at the 

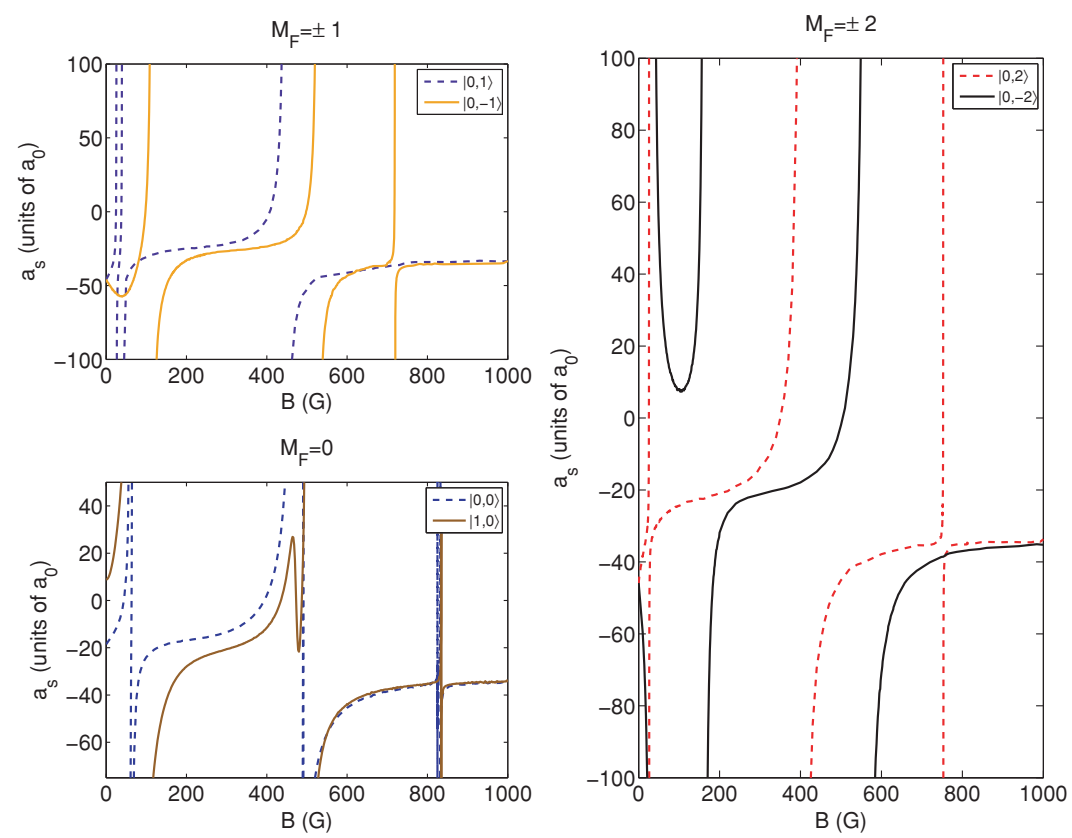

FIG. 4. (Color online) Scattering lengths $a_{s}$ ( $s$-wave) as a function of the magnetic field strength $B$. The top left panel refers to channels with $\left|M_{F}\right|=1$, the bottom left panel shows channels with $M_{F}=0$, whereas channels with $\left|M_{F}\right|=2$ are plotted in the right panel. Notice the two narrow Feshbach resonances in the $|0,1\rangle$ channel at magnetic field strengths ( $B \simeq 25-40 \mathrm{G}$ ), visible in the upper left panel. Two narrow Feshbach resonances are also found in the $|0,0\rangle$ channel at the magnetic field strengths $B=50-60 \mathrm{G}$, less visible in the bottom left panel. There is also a narrow Feshbach resonance in the channel $|0,-2\rangle$ at $B \simeq 30 \mathrm{G}$, visible in the right panel where the solid line diverges to $-\infty$. See Table II for further details regarding the Feshbach resonances.

center of the resonance, and $a_{\mathrm{bg}}$ is the background scattering length, that is, the scattering length away from the resonance where $B-B_{0}$ is large. To further characterize the calculated Feshbach resonances we report $a_{\mathrm{bg}}, \Delta$, and $B_{0}$ in Table II.

Furthermore, we calculate the $s$-wave scattering lengths $a_{s}(B)$ for channels corresponding to the $F_{1}=1, F_{2}=2$ hyperfine manifold, that is, collisions between atomic states $\left|F_{1} M_{F_{1}}\right\rangle_{A}$ and $\left|F_{2} M_{F_{2}}\right\rangle_{A}$ with $F_{1}=1$ and $F_{2}=2$. Selected results are collected in the top panel of Fig. 5. Four channels are chosen to depict the typical behavior of $a_{s}(B)$ for this group of channels. Among them are the two channels $|0, \pm 3\rangle$ accessible through collisions between atoms in the atomic hyperfine states $|1, \pm 1\rangle_{A}$ and $|2, \pm 2\rangle_{A}$. Whereas the $|0,-3\rangle$ and $|0,3\rangle$ channels have an almost pure triplet character and therefore attain the background triplet scattering length $\simeq-34 a_{0}$, this is not seen for the two channels $|2,2\rangle$ and $|3,0\rangle$. Collisions between atoms in the atomic hyperfine states $|1,0\rangle_{A}$ and $|2,2\rangle_{A}$ are represented by the channel $|2,2\rangle$, whereas collisions between atoms in the states $|1,0\rangle_{A}$ and $|2,0\rangle_{A}$ are represented by the channel $|3,0\rangle$. The scattering length $a_{S}(B)$ for channel $|3,0\rangle$, depicted in the top panel of Fig. 5, show a resonancelike structure. This is not a Feshbach resonances in the sense that we have previously discussed. The $s$-wave scattering length does not diverge to $\pm \infty$. Instead, $a_{s}(B)$ evolves rapidly and changes sign at $B \simeq 257 \mathrm{G}$, but $a_{s}(B)$ never exceeds $250 a_{0}$ and never goes below $-600 a_{0}$. We remark that this is not due to the finite resolution in our calculations.

To complete the discussion, we report the $s$-wave scattering length $a_{S}(B)$ for collisions between two atoms in the identical hyperfine states $\left|2, M_{F_{1}}\right\rangle_{A}$, with $M_{F_{1}}=M_{F_{2}}=0, \pm 1, \pm 2$. The results are shown in the bottom panel of Fig. 5. The selected channels are chosen to give a flavor of the typical behavior for $a_{S}(B)$ for this group of channels.

Channels with $M_{F}= \pm 3, \pm 4$ have $s$-wave scattering lengths which attain the background triplet scattering length because of their triplet character. Additional channels have their scattering lengths determined by the triplet and singlet scattering lengths and vary slowly and continuously between the two. This type of behavior is exemplified by the channels $|2,2\rangle$ (top panel) and $|4,-2\rangle$ (bottom panel) in Fig. 5. The Zeeman shifts drives these channels to either a dominantly singlet or triplet character as the magnetic field strength increases.

As discussed previously, the Feshbach resonances evident in Fig. 4 arise because the energy of the atom pair coincides in energy with that of a bound molecular state. By calculating the bound molecular levels, it is possible to study this relationship in more detail (see Fig. 6). The energy of a bound molecular state corresponding to zero rotation is determined from the vibrational energy together with the hyperfine and Zeeman interactions. The singlet and triplet character of the bound 
TABLE II. Characterization of Feshbach resonances found in channels corresponding to the lowest hyperfine level, that is, with $F_{1}=F_{2}=1$. The second column shows experimentally measured values for the calculated Feshbach resonances. Experimental data are from D'Errico et al. $B_{\text {theory }}$ denotes the theoretical predictions for the center of the different Feshbach resonances. Referring to Eq. (15), $B_{\text {theory }}$ should be used for $B_{0}$ in the parametrization of these resonances. See the text for a discussion of the column with heading $S, I, F$. The background scattering length $a_{\mathrm{bg}}$ [cf. Eq. (15)] are given in units of $a_{0}$. Finally, $\Delta$ denotes the theoretical predictions for the resonance widths, measured in gauss. In addition to their experimental observations, D'Errico et al. [11] performed theoretical calculations of resonances in the channels $|0, \pm 2\rangle$ and $|0,0\rangle$. Their results are in good agreement with those of the present work.

\begin{tabular}{lccccc}
\hline \hline$\left|n M_{F}\right\rangle$ & $B_{\text {expt }}(\mathrm{G})$ & $B_{\text {theory }}(\mathrm{G})$ & $S, I, F$ & $a_{\text {bg }}\left(\right.$ units of $\left.a_{0}\right)$ & $\Delta(\mathrm{G})$ \\
\hline$|0,2\rangle$ & $25.85 \pm 0.10$ & 25.90 & $1,3,3$ & -35.73 & -0.4315 \\
$|0,2\rangle$ & $403.4 \pm 0.7$ & 402.9 &,,--- & -29.48 & -51.39 \\
$|0,2\rangle$ & $752.3 \pm 0.1$ & 752.8 & & -35.29 & -0.3971 \\
$|0,-2\rangle$ & $32.6 \pm 0.15$ & 33.61 & $1,1,(2)$ & -13.46 & 79.82 \\
$|0,-2\rangle$ & $162.89 \pm 0.9$ & 162.5 & $1,3,(3)$ & -11.75 & -60.58 \\
$|0,-2\rangle$ & $562.2 \pm 0.15$ & 561.4 & $0,-,(3)$ & -29.19 & -55.22 \\
$|0,1\rangle$ & & 25.80 & $1,3,3$ & -38.02 & -1.252 \\
$|0,1\rangle$ & & $1,1,1$ & -38.46 & -2.196 \\
$|0,1\rangle$ & & $(0),-,-$ & -30.15 & -37.82 \\
$|0,-1\rangle$ & & $1,3,-$ & -25.75 & -29.48 \\
$|0,-1\rangle$ & 113.9 &,,-- 3 & -30.90 & -28.39 \\
$|0,-1\rangle$ & 526.4 & & -36.94 & -0.9820 \\
$|0,0\rangle$ & & 719.0 & $1,3,3$ & -15.92 & -10.45 \\
$|0,0\rangle$ & & $1,1,-$ & -21.09 & -5.403 \\
$|0,0\rangle$ & & $(0), 2,1$ & -22.56 & -88.11 \\
$|0,0\rangle$ & 66.49 & $(0), 3,-$ & -80.11 & -12.37 \\
$|0,0\rangle$ & & & -32.41 & -0.0361 \\
$|0,0\rangle$ & & & -36.31 & -0.5249 \\
$|1,0\rangle$ & & & -17.23 & -150.9 \\
$|1,0\rangle$ & $66.0 \pm 0.9$ & 891.4 & $1,3,3$ & -22.31 \\
\hline \hline
\end{tabular}

molecular levels is in part lost for these highly excited vibrational levels, as the hyperfine interaction couples the
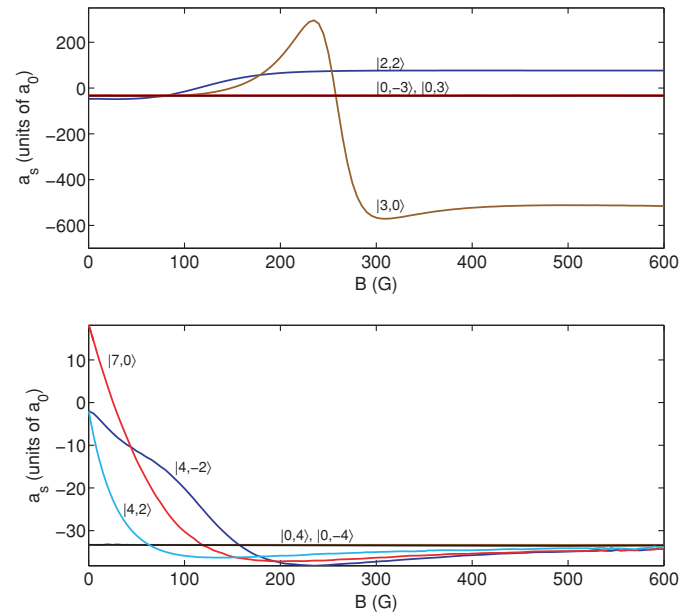

FIG. 5. (Color online) Calculated $s$-wave scattering lengths as a function of the magnetic field. (Top) Selected channels from the $F_{1}=1, F_{2}=2$ hyperfine manifold. Notice the large variation in the scattering length for the $|3,0\rangle$ channel. (Bottom) Scattering lengths for atoms with $F_{1}=F_{2}=2$ and $M_{F_{1}}=M_{F_{2}}=0, \pm 1, \pm 2$ (at zero magnetic field) corresponding to the channels $|7,0\rangle,|4, \pm 2\rangle$, and $|0, \pm 4\rangle$, respectively. electronic states ${ }^{1} \Sigma_{g}^{+}$and ${ }^{3} \Sigma_{u}^{+}$. Thus, $S$ is in general not a good quantum number in this regime.

We start from pure bound singlet and triplet vibrational states and introduce the hyperfine and Zeeman interactions. To analyze the weakly bound molecular levels, we need only a few parameters. These are the molecular hyperfine parameters, the rotational constant $B$, and the vibrational energies for both the singlet and the triplet states. The hyperfine parameters are very well approximated by the atomic values for such highly excited states. A reasonable value for the rotational constant can be easily calculated. The vibrational energies are unknown, but approximate values can be obtained from the resonance positions. For the most weakly bound singlet and triplet vibrational energies, we find the values $\simeq-1590$ and $\simeq-510 \mathrm{MHz}$, respectively. The energy is measured relative to the disassociation limit for two free atoms without hyperfine structure at $B=0$.

Through the hyperfine interaction the triplet and the singlet vibrational levels interact and this system holds bound molecular levels. Depending on the interaction, Feshbach molecules in these loosely bound molecular levels can be characterized more or less by the quantum numbers $S, I$, and $F$. In Table II we have included these quantum numbers whenever it is meaningful to do so. Blank entries signals that the corresponding resonances cannot be fully explained within this simple two-vibrational model; that is, one needs to include also additional vibrational levels. Finally, we remark that the quantum numbers $S, I$, and $F$ are quantum numbers for the bound Feshbach molecules, not for the open scattering channels. 

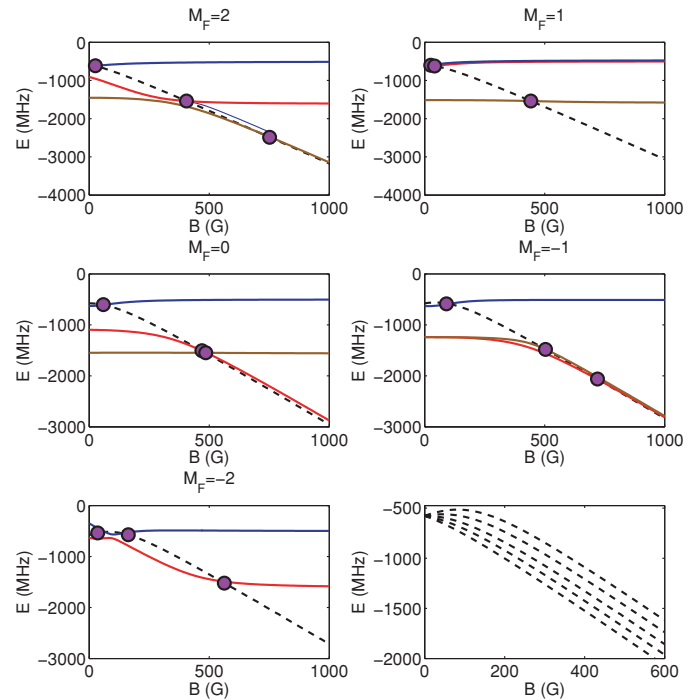

FIG. 6. (Color online) Channel states (dashed black line) vs weakly bound molecular states (red, black, and brown solid lines) as a function of the magnetic field strength for different values of $M_{F}$. The energy is given in $\mathrm{MHz}$ relative to the dissociation limit for two free atoms without hyperfine structure. Intersections between the channel state energies and that of the bound molecular states are shown as filled circles. These are Feshbach resonances. (Bottom right) The channel state energies as a function of the magnetic field for all channels $\left|0, M_{F}\right\rangle$ included in the other panels. The top line is for the channel with $M_{F}=-2$, followed by channels with $M_{F}=-1$, 0,1 , and 2 .

Figure 7 shows the quantum numbers $S, I$, and $F$ as a function of the magnetic field for three bound molecular states with $M_{F}=2$. At zero magnetic field, the total nuclear spin $I$ and the total angular momentum $F$ are good quantum numbers, while the total electronic spin $S$ is not, due to the hyperfine singlet-triplet interaction.

\section{B. Feshbach resonances and $s$-wave scattering lengths for the ${ }^{41} K_{2}$ system}

The potassium isotope ${ }^{41} \mathrm{~K}$ has nuclear spin $I=\frac{3}{2}$, which is identical to the nuclear spin of the ${ }^{39} \mathrm{~K}$ isotope. The difference between the two systems ${ }^{39} \mathrm{~K}_{2}$ and ${ }^{41} \mathrm{~K}_{2}$ are then: (i) the reduced mass and (ii) the hyperfine splitting. The molecular Fermi contact parameters $b_{f}$ and $b_{f}^{\prime}$ determine the strength of the hyperfine interaction. These parameters are obtained from the atomic hyperfine splitting [25] and the result is $b_{f}=63.5035 \mathrm{MHz}=-b_{f}^{\prime}$. A large Fermi-contact parameter $b_{f}$ implies a strong coupling between the electronic states ${ }^{3} \Sigma_{u}^{+}$and ${ }^{1} \Sigma_{g}^{+}$and vice versa. Generally, a weak coupling gives narrow Feshbach resonances, whereas a strong coupling leads to broad Feshbach resonances.

The $s$-wave scattering lengths are calculated for all channels $\left|n, M_{F}\right\rangle$ corresponding to the $F_{1}=F_{2}=1$ hyperfine manifold, that is, two colliding atoms in the atomic hyperfine states $\left|1, M_{F_{1}}\right\rangle$ and $\left|1, M_{F_{2}}\right\rangle$. In the right panel of Fig. 8 the $s$-wave scattering lengths $a_{s}(B)$ as functions of the magnetic field strength are shown for the channels $|0,2\rangle$. These channels represent collisions between atoms in the atomic hyperfine states $\left|F_{1} M_{F_{1}}\right\rangle_{A}=|1,1\rangle_{A}$ and $\left|F_{1} M_{F_{2}}\right\rangle=|1,1\rangle_{A}$. In total there are six Feshbach resonances, four in the $M_{F}=-2$ channel and two in the $M_{F}=2$ channel. All the resonances are rather narrow compared to the resonances in the ${ }^{39} \mathrm{~K}$ isotope. Note that there may exist additional resonances that are not resolved, resonances with a width below $0.01 \mathrm{G}$ could go undetected. To the best of our knowledge, none of the calculated resonances for ${ }^{41} \mathrm{~K}$ have been observed. There are, however, other theoretical predictions [11] for the resonances in the channels $|0, \pm 2\rangle$.

Furthermore, there are several Feshbach resonances in the $|0, \pm 1\rangle$ channels, seen in Fig. 8. Two resonances are in the $|0,-1\rangle$ channel and one is seen in the $|0,1\rangle$ channel. These resonances are accessible in experiments where two atoms in the atomic hyperfine states $|1,0\rangle_{A}$ and $|1, \pm 1\rangle_{A}$ are allowed to collide, the \pm sign is for the $|0, \pm 1\rangle$ channel.

For the two channels with $M_{F}=0$ we find three resonances, one in the $|0,0\rangle$ channel and two in the $|1,0\rangle$ channel, shown in Fig. 8. The resonances in the $|0,0\rangle$ channel correspond to collisions between atoms in the identical hyperfine state $|1,0\rangle_{A}$, whereas the resonances in the $|1,0\rangle$ channel can be realized form collisions between atoms in the atomic hyperfine states $|1,-1\rangle_{A}$ and $|1,1\rangle_{A}$. At some magnetic field strengths Fig. 8 is somewhat misleading, with reference the bottom left panel. At $B \simeq 750 \mathrm{G}$, the scattering length for the $|1,0\rangle$ channel (dashed brown line) seems to diverge to $\pm \infty$ twice. However, this is due to the range on the $y$ axis, the $s$-wave scattering length diverges only once, but has a rapid variation for magnetic field strengths in the vicinity of the resonance position. Thus, Fig. 8 and Table III should be compared.

TABLE III. Feshbach resonances in $s$-wave channels for all channels with quantum numbers $F_{1}=F_{2}=1$ at low magnetic field strengths. The results included in this table are for the ${ }^{41} \mathrm{~K}$ isotope. The background scattering lengths are included in column 3 , given in units of $a_{0}$. The resonances widths $\Delta$ are included in column 4 . To our best knowledge none of the calculated resonances have been observed. However, D'Errico et al. [11] used their experimental observations to optimize a model from which they obtained theoretical predictions for Feshbach resonances in the channels $|0, \pm 2\rangle$ and $|0,0\rangle$. Their values are in good agreement with the present work.

\begin{tabular}{lccc}
\hline \hline$\left|n M_{F}\right\rangle$ & $B_{\text {theory }}(\mathrm{G})$ & $a_{\text {bg }}\left(\right.$ units of $\left.a_{0}\right)$ & $\Delta(\mathrm{G})$ \\
\hline$|0,2\rangle$ & 409.6 & 60.48 & 0.007775 \\
$|0,2\rangle$ & 661.2 & 60.50 & 0.2212 \\
$|0,-2\rangle$ & 51.17 & 64.74 & -0.3623 \\
$|0,-2\rangle$ & 500.8 & 60.41 & 0.004612 \\
$|0,-2\rangle$ & 748.1 & 60.56 & 0.2237 \\
$|0,-2\rangle$ & 947.4 & 59.90 & 0.002226 \\
$|0,1\rangle$ & 675.1 & 60.49 & 0.1558 \\
$|0,-1\rangle$ & 51.95 & 63.29 & -0.09760 \\
$|0,-1\rangle$ & 717.6 & 60.34 & 0.1184 \\
$|0,0\rangle$ & 452.4 & 60.15 & 0.01176 \\
$|0,0\rangle$ & 703.8 & 60.31 & 0.2962 \\
$|1,0\rangle$ & 455.8 & 60.52 & 0.0003994 \\
$|1,0\rangle$ & 685.6 & 60.94 & 0.1165 \\
\hline \hline
\end{tabular}



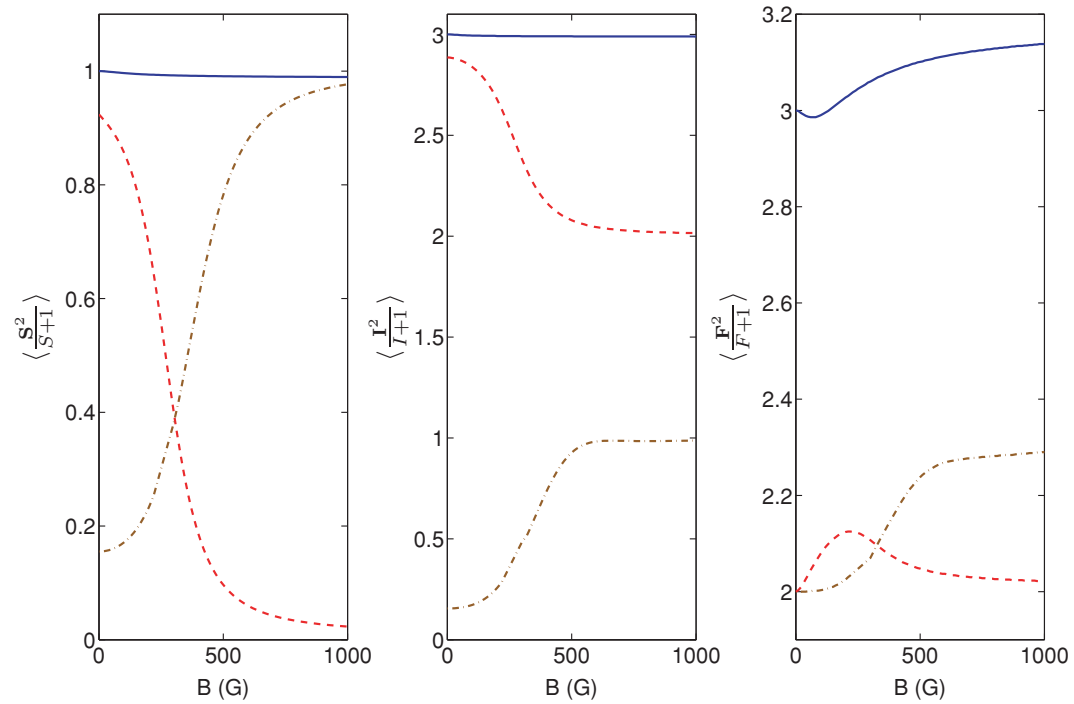

FIG. 7. (Color online) Expectation values $\langle S\rangle,\langle I\rangle$, and $\langle F\rangle$ as a function of the magnetic field for three weakly bound states with total angular momentum $M_{F}=2$. These are identical with the bound molecular states shown in the top left panel of Fig. 6 . The top line in the top left panel of Fig. 6 denotes the same state as the solid lines in this figure, whereas the bottom line in the same panel of Fig. 6 corresponds to the dash-dotted line in this figure. Note the definition $\langle S\rangle \equiv\left\langle\frac{\hat{S}^{2}}{(S+1)}\right\rangle$, and similarly for $\langle I\rangle$ and $\langle F\rangle$. See the text for a further discussion.
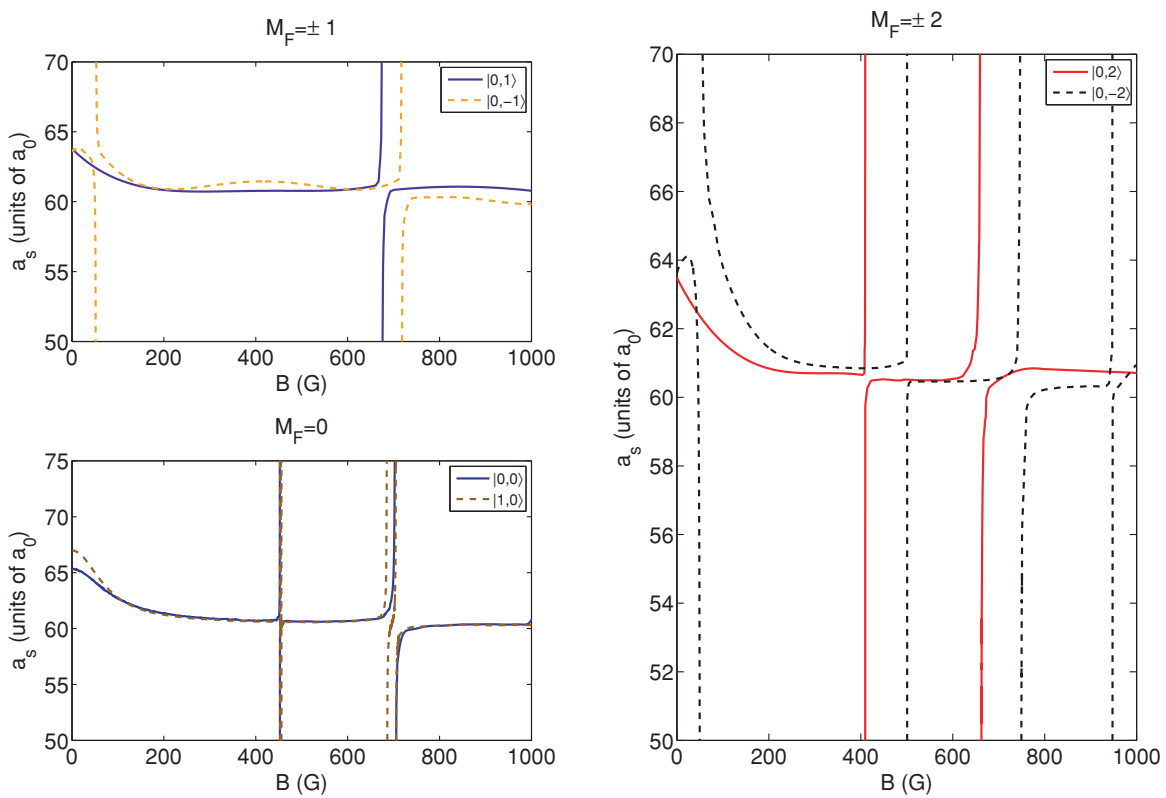

FIG. 8. (Color online) Scattering lengths ( $s$-wave) as a function of the magnetic field strength for ${ }^{41} \mathrm{~K}_{2}$. The top left panel refers to channels with $\left|M_{F}\right|=1$, the bottom left panel shows channels with $M_{F}=0$; channels with $\left|M_{F}\right|=2$ are plotted in the right panel. See the text for a further discussion. 
Apart from the resonances, the $s$-wave scattering length behaves smoothly as it decreases from $65 a_{0}$ at $B=0 \mathrm{G}$ to $60 a_{0}$ at $B=1000 \mathrm{G}$. For comparison, the background triplet scattering length is $60.50 a_{0}$ while the singlet scattering length is somewhat larger and equal to $85.49 a_{0}$. From the calculated scattering lengths $a_{s}(B)$ reported in Fig. 8, it seems that the majority of channels have scattering lengths comparable to the triplet scattering length. Hence, most channels in the system appear to have a triplet character away from resonances. By calculating $\langle S\rangle$ as a function of the magnetic field strength for the relevant channels, this hypothesis is confirmed (top panel of Fig. 9). At high magnetic field strengths $S$ is a good quantum number and $S=1$. From the bottom panel of Fig. 9, where $\langle I\rangle$ is calculated as a function of the magnetic field, we observe that $I$ is generally not a good quantum number.

Table III provides more detailed information about each Feshbach resonance, its width and background scattering length, in accordance with Eq. (15). We have performed additional calculations of the $s$-wave scattering lengths for the selected channels $|4, \pm 2\rangle,|7,0\rangle$, and $|0, \pm 4\rangle$. These channels correspond to collisions between atoms in the identical atomic hyperfine states $|2, \pm 1\rangle_{A},|2,0\rangle_{A}$, and $|2, \pm 2\rangle_{A}$. In addition to the channels we have discussed previously, these are some of the most important channels, experimentally. The results are shown in Fig. 10. Channels with $M_{F}= \pm 4$ are again seen to have very predictable $s$-wave scattering lengths.

\section{Transition rates}

In this section we will investigate how cold atomic collisions can change the hyperfine states of the collision partners. Two atoms in the initial states $\left|F_{1} M_{F_{1}}\right\rangle_{A}$ and $\left|F_{2} M_{F_{2}}\right\rangle_{A}$ collide and the final atomic states are $\left|F_{1}^{\prime} M_{F_{1}}^{\prime}\right\rangle_{A}$ and $\left|F_{2}^{\prime} M_{F_{2}}^{\prime}\right\rangle_{A}$. As we discussed earlier, the sum $M_{F}=M_{F_{1}}^{\prime}+M_{F_{2}}^{\prime}=M_{F_{1}}+M_{F_{2}}$ is conserved in such a collision.
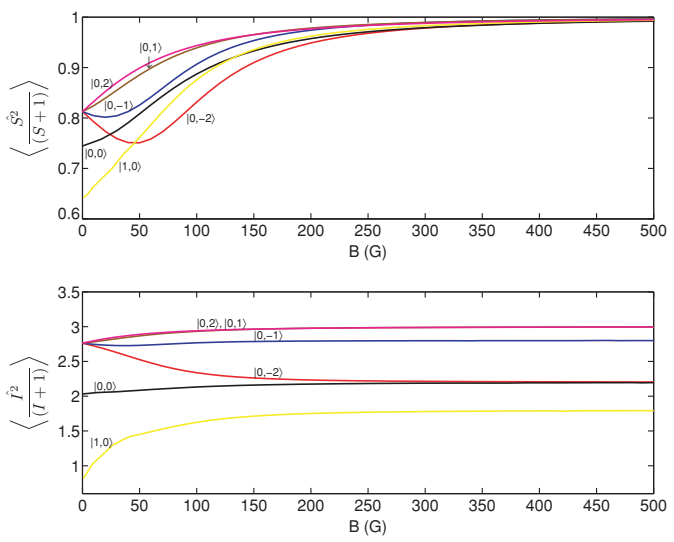

FIG. 9. (Color online) $\langle S\rangle$ (top) and $\langle I\rangle$ (bottom) shown as a functions of the magnetic field strength for channels corresponding to two atoms with zero orbital angular momentum $(l=0)$ and hyperfine quantum numbers $F_{1}=F_{2}=1$. Note that $\langle S\rangle \equiv\left\langle\frac{\hat{S}^{2}}{(S+1)}\right\rangle$, and similarly for $\langle I\rangle$.

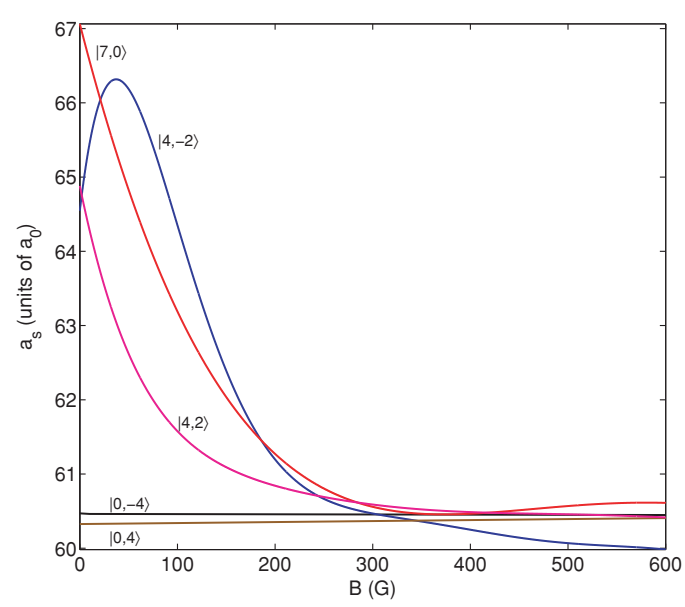

FIG. 10. (Color online) Scattering lengths ( $s$-wave) as a function of the magnetic field strength for collisions between atoms in identical atomic hyperfine states $\left|2, M_{F_{1}}\right\rangle_{A}$.

We report results for the transition rate constants, previously defined in Eq. (12), for homonuclear collisions between two potassium atoms. It is clear from the definition in Eq. (12) that the transition rate constants can be readily obtained once the important $S$ matrix has been calculated. In atomic or molecular traps where the density is high, collisions between the particles are of great importance. When the relevant rate constants are known, predictions can be made for the lifetime of a trapped sample of atoms or molecules, as we will show in an example. Magnetic trapping relies on the system to be in a trappable state, that is, a state where the Zeeman shift is favorable for the relevant experimental setup. Transitions between hyperfine states may then lead to trap loss whenever a transition drives the system into an untrappable state. In addition, an amount of internal energy will be transformed to kinetic energy in a transition between hyperfine states. Furthermore, the population of hyperfine states changes over time due to two-body collisions. After some time, a steadystate population of hyperfine states is established. The time scale for this to happen can be estimated from the transition rate constants, as we will demonstrate. In the past, and particular in relation to the experiments leading to $\mathrm{BEC}$, the transition rates for hydrogen have been thoroughly investigated (see for instance [28,29]). Tiesinga et al. studied collisions between sodium atoms [30] and Houbiers et al. [31] reported rates for collisions between lithium atoms.

As previously mentioned, the number of atoms lost from an atomic trap due to hyperfine state-changing collisions can be estimated. Knowledge of which processes lead to trap loss is a necessity, together with the corresponding rates $G_{a \rightarrow b}$. Once these pieces of information are obtained, the decrease in density due to atoms escaping the trap per time is given as [19]

$$
\frac{d \rho}{d t}=-\rho^{2} \sum_{i} g_{i} G_{i}\left(a_{i} \rightarrow b_{i}\right),
$$


where $G_{i}\left(a_{i} \rightarrow b_{i}\right)$ are the rate constants for the transitions $\left|a_{i}\right\rangle \rightarrow\left|b_{i}\right\rangle$ and the scalars $g_{i}$ are the number of atoms lost in transition $i$. The density $\rho$ is given as $\rho=N / V$, where $V$ is the volume of the trap. Correspondingly, the density decays over a time scale $T_{\text {loss }}$ given as

$$
T_{\mathrm{loss}}=\frac{1}{\rho \sum_{i} g_{i} G\left(a_{i} \rightarrow b_{i}\right)} .
$$

The population of different atomic hyperfine states within the trap can also be studied. Initially, all atoms present in the trap might be in just one single hyperfine state. As hyperfine changing collisions occur, additional states will be populated. After some time of the order $T_{\mathrm{ex}}$, a range of different atomic hyperfine states coexist within the trap. This is a result of transitions $|a\rangle \rightarrow|b\rangle$, where both $|a\rangle$ and $|b\rangle$ represent trappable states. An estimate for $T_{\mathrm{ex}}$ can be calculated once the transition rates are known as

$$
T_{\mathrm{ex}}=\frac{1}{\rho \sum_{i} G_{i}\left(a_{i} \rightarrow b_{i}\right)} .
$$

$T_{\text {ex }}$ is only relevant when it is much smaller than $T_{\text {loss }}$.

For each of the two potassium isotopes considered in the present work, there are in total 36 relevant channels with zero orbital angular momentum, which means that the number of allowed transitions is as high as 80 . See also Fig. 3, where all channels are defined together with their Zeeman shifts. Due to this multitude of possible transitions, we will in the present work only present calculations for some selected cases.

The transition rate constants $G_{a \rightarrow b}$ depend on the magnetic field, and we report $G_{a \rightarrow b}(B)$ for $B \in[10,1000] \mathrm{G}$.

The complete Hamiltonian for the system is written $\hat{H}=\hat{H}_{\mathrm{Z}}+\hat{H}_{\mathrm{ev}}+\hat{H}_{\mathrm{hf}}+\hat{H}_{\mathrm{rot}}$, but the rotational and Zeeman interactions are diagonal in the channel basis and do not couple different channels. The hyperfine interaction $\hat{H}_{\mathrm{hf}}$ is in general not diagonal in the channel basis. This is because the complete molecular hyperfine interaction depends on the internuclear separation $r$ through the molecular hyperfine parameters $b_{f}(r), c(r), b_{f}(r)^{\prime}$, and $c(r)^{\prime}$ introduced and defined in [15]. When $r \rightarrow \infty$ the molecular hyperfine interaction correlates with the well-known atomic hyperfine interaction which does not couple different channel states. Unfortunately, values for the molecular hyperfine parameters are not available and we will therefore use the atomic hyperfine interaction for all values of $r$. This is a valid approximation as the distance between the collision partners is large due to the low temperature. Hence, the transitions we study are induced by the electronic Hamiltonian $\hat{H}_{\mathrm{ev}}$. This means that the total angular momentum projection $M_{F}=M_{F_{1}}+M_{F_{2}}+m_{l}$ is conserved in a transition. The electronic Hamiltonian is most effective in coupling channels with the same expectation values for the total angular momentum $\hat{\mathbf{F}}^{2}$ and the total electronic spin $\hat{\mathbf{S}}^{2}$ operators. The channel states are not eigenstates for the $\hat{\mathbf{S}}^{2}$ operator, nor for the total angular momentum operator $\hat{\mathbf{F}}^{2}$ in a magnetic field. A channel state $|\psi\rangle$ takes the general form

$$
|\psi\rangle=\sum_{i} c_{i}\left|q \Lambda S I F M_{F}\right\rangle_{i},
$$

where $|q \Lambda S\rangle_{i}$ are Born-Oppenheimer states, that is, eigenstates for the $\hat{H}_{\mathrm{ev}}$ operator, and the state $\left|q \Lambda S I F M_{F}\right\rangle$ is the result of coupling the nuclear spin states $\left|I M_{I}\right\rangle$ to the Born-Oppenheimer states $(\mathbf{F}=\mathbf{S}+\mathbf{I})$. We consider collisions without orbital angular momentum, hence the molecular rotational quantum number is zero. The coupling element $\left\langle\psi_{1}\left|\hat{H}_{\mathrm{ev}}\right| \psi_{2}\right\rangle$ takes the form

$$
\begin{aligned}
& \left\langle\psi_{1}\left|\hat{H}_{\mathrm{ev}}\right| \psi_{2}\right\rangle \\
& \quad=\sum_{i j} c_{i} c_{j} V_{i}(r)\left\langle q_{i} \Lambda_{i} S_{i} I_{i} F_{i} M_{F} \mid q_{j} \Lambda_{j} S_{j} I_{j} F_{j} M_{F}\right\rangle,
\end{aligned}
$$

a useful relation for discussing transition rates. $V_{i}(r)$ are the Born-Oppenheimer potentials defined in Sec. III. In Fig. 11 we report transition rates for transitions between channels with the largest positive Zeeman shifts to the largest negative Zeeman shifts for states with $M_{F}=2,1,-1,-2$. Results for both isotopes are included. These transitions represent collisions between atoms in an initial state with $F_{1}=F_{2}=2$ (low magnetic field quantum numbers) and with large positive Zeeman shifts. The final postcollision atomic states correspond to the $F_{1}=F_{2}=1$ hyperfine manifold (low magnetic field quantum numbers) with large negative Zeeman shifts (see also Fig. 3). Note that there is a significant conversion of internal energy to kinetic energy during these collisions. The difference between the isotopes is due to the different hyperfine splittings; the mass difference is too small to have a significant impact on the rates.

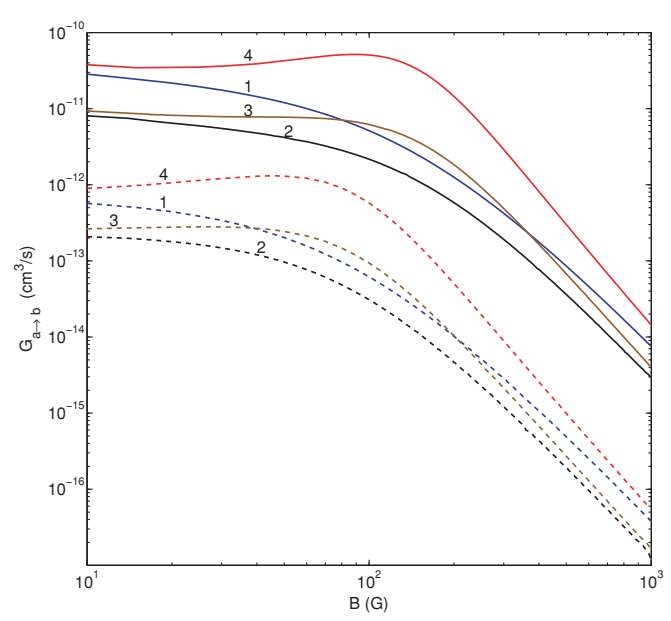

FIG. 11. (Color online) Transition rates $G_{a \rightarrow b}$ as a function of the magnetic field. Included are the following transitions: $1,|4,2\rangle \rightarrow$ $|0,2\rangle ; 2,|5,1\rangle \rightarrow|0,1\rangle ; 3,|5,-1\rangle \rightarrow|0,-1\rangle ;$ and $4,|4,-2\rangle \rightarrow$ $|0,-2\rangle$ between channels designated by $\left|n, M_{F}\right\rangle$. All rates are for collisions between atoms with initial state $\left|F_{1}=2, M_{F_{1}} F_{2}=2, M_{F_{2}}\right\rangle$ and with final state $\left|F_{1}=1, M_{F_{1}}, F_{2}=1, M_{F_{1}}\right\rangle_{A}$ (low-field quantum numbers). Hence, these transitions are important as they take the atoms from low-field-seeking states to high-field-seeking states. See Table I for the complete set of $M_{F_{1}}$ and $M_{F_{2}}$ quantum numbers involved in the processes. Solid lines represent the ${ }^{39} \mathrm{~K}$ isotope, whereas dashed lines are for the ${ }^{41} \mathrm{~K}$ isotope. The initial and final channels have the same value of $M_{F}=M_{F_{1}}+M_{F_{2}}$. See the text for further discussion. 


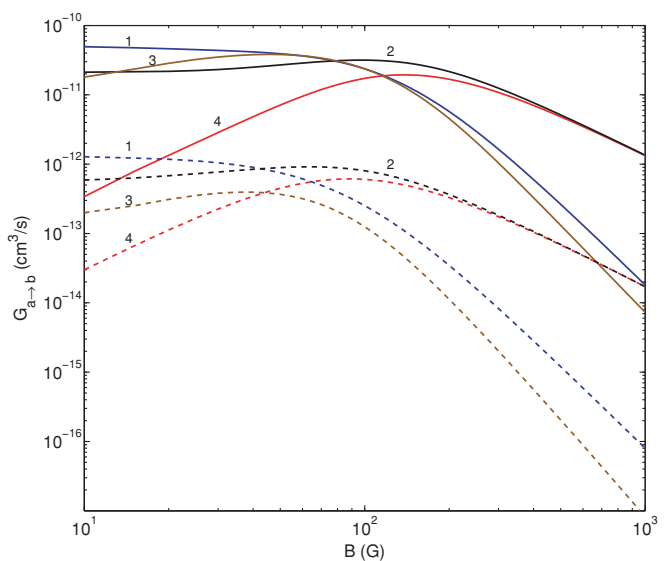

FIG. 12. (Color online) Transition rates $G_{a \rightarrow b}$ as a function of the magnetic field for transitions $|7,0\rangle \rightarrow|n, 0\rangle$. Included are the following transitions: $1,|7,0\rangle \rightarrow|0,0\rangle ; 2,|7,0\rangle \rightarrow|4,0\rangle ; 3$, $|7,0\rangle \rightarrow|6,0\rangle$; and $4,|7,0\rangle \rightarrow|3,0\rangle$. Solid lines represent the ${ }^{39} \mathrm{~K}$ isotope, whereas dashed lines are for the ${ }^{41} \mathrm{~K}$ isotope.

Figure 12 shows additional transition rates among the $M_{F}=0$ channels. We consider the transition rates from the $|7,0\rangle$ channel, with the low-field quantum numbers $|2,0,2,0\rangle_{A}$, that is, a channel with two atoms in the identical hyperfine state $|2,0\rangle_{A}$. Included in the figure are the rates for the four most dominant processes from this initial channel to a final state, specified by the low-field quantum numbers: $|1,0,1,0\rangle,|1,-1,2,1\rangle,|2,-1,2,1\rangle$, and $|1,0,2,0\rangle$ for transitions $1,2,3$, and 4 , respectively. Other transitions induced by collisions from the specified initial state are possible, but not likely, as the transition rates for such processes are at least one order of magnitude smaller than for the processes included here.

Finally, we include in Fig. 13 the dominant transition rates at large magnetic fields, for all values of $M_{F}$. A rather typical feature is that one or two processes have transition rates that are an order of magnitude larger than all the other transitions at large magnetic field strengths. This effect generally tends to stem from the strong channel coupling due to the electronic Hamiltonian $\hat{H}_{\mathrm{ev}}$. Characteristic for the expansions [cf. Eq. (19)] of the initial and final channels involved in the strong transitions is the fact that they have the same set of dominant terms, which leads to large matrix elements of $\hat{H}_{\mathrm{ev}}$. The Zeeman interaction also plays an important role. Although it does not couple the two channels directly, the Zeeman interaction affects the channel states and changes their expectation values $\langle S\rangle,\langle I\rangle$, and $\langle F\rangle$, that is, the coefficients in Eq. (19). This makes some transitions more favorable as the matrix element for the $\hat{H}_{\mathrm{ev}}$ operator increases, while other transitions are suppressed with only negligible coupling elements.
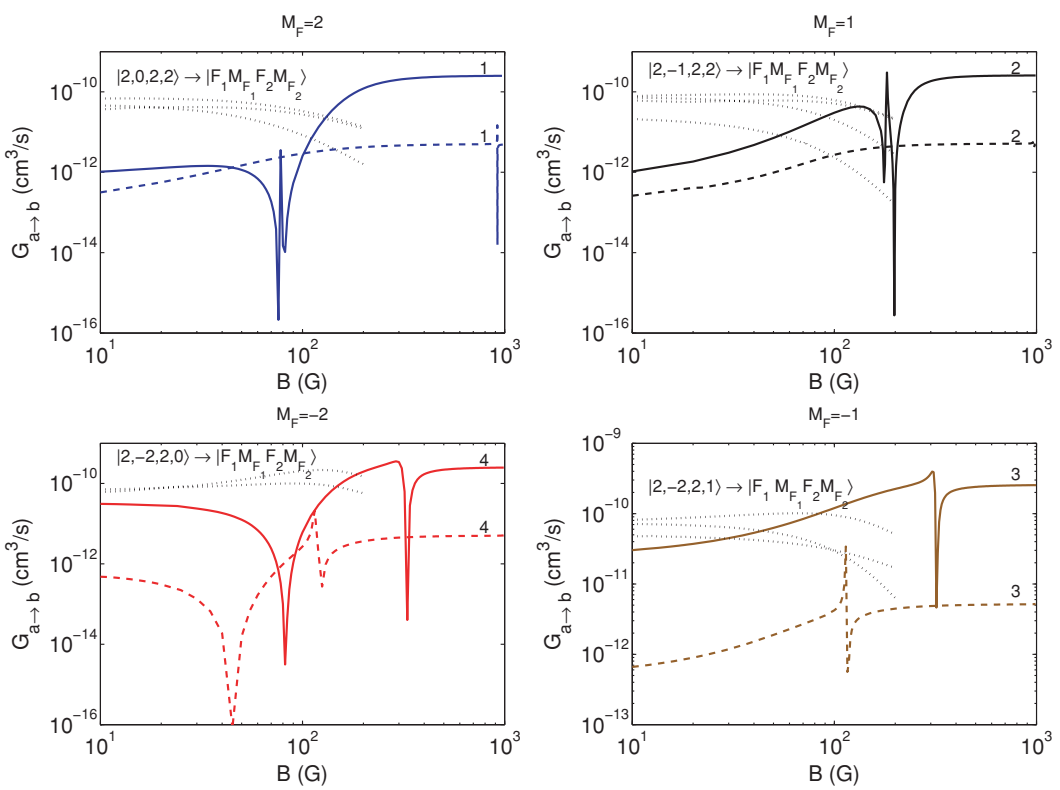

FIG. 13. (Color online) Transition rates $G_{a \rightarrow b}$ as a function of the magnetic field. Included are the following transitions: 1, $|2,2\rangle \rightarrow|1,2\rangle$ (blue, top left); $2,|3,1\rangle \rightarrow|1,1\rangle$ (black, top right); $3,|4,-1\rangle \rightarrow|2,-1\rangle$ (red, bottom left); and $4,|3,-2\rangle \rightarrow|2,-2\rangle$ (brown, bottom right) between channels $\left|n, M_{F}\right\rangle$. Solid lines represent the ${ }^{39} \mathrm{~K}$ isotope, whereas dashed lines represent the ${ }^{41} \mathrm{~K}$ isotope. We have also included dotted black lines which show the transition rates for a group of transitions that are dominant at low magnetic-field strengths. These rates decrease rapidly at high magnetic field strengths, as indicated in the individual panels. The included dotted lines are for the ${ }^{39} \mathrm{~K}$ isotope. Several resonances are observed in the figure. Their rather subtle origins will, however, not be discussed here. 
The dominant transitions shown in Fig. 13 involve small amounts of energy; the Zeeman shifts of the initial and the final channels are very similar. In terms of the low-field quantum numbers, transition 1 is expressed as $|1,1,2,1\rangle \rightarrow|1,0,2,2\rangle$, while transition 2 is $|1,-1,2,0\rangle \rightarrow|1,1,2,-2\rangle$. Hence, only the individual values of $M_{F_{1}}$ and $M_{F_{2}}$ change in these collisions, keeping the sum $M_{F_{1}}+M_{F_{2}}$ constant. Low-field quantum numbers for transitions 3 and 4 are $|2,-2,2,1\rangle \rightarrow$ $|1,0,2,-1\rangle$ and $|2,-2,2,0\rangle \rightarrow|1,-1,2,-1\rangle$, respectively. The initial channels correspond to the $F_{1}=F_{2}=2$ hyperfine manifold, whereas the final channel corresponds to the $F_{1}=1, F_{2}=2$ manifold.

As an example, consider an atomic trap filled with low-fieldseeking ${ }^{39} \mathrm{~K}$ atoms. The atoms are prepared in identical atomic hyperfine states $|2,1\rangle_{A}$, such that $M_{F}=M_{F_{1}}+M_{F_{2}}=2$, and the collision partners are described by the channel state $|4,2\rangle$ prior to collisions within the trap. Furthermore, we assume an initial density $\rho=10^{12} \mathrm{~cm}^{-3}$. The possible transitions from the channel $|4,2\rangle$ are $1,|4,2\rangle \rightarrow|0,2\rangle ; 2,|4,2\rangle \rightarrow|1,2\rangle ; 3$, $|4,2\rangle \rightarrow|2,1\rangle$; and $4,|4,2\rangle \rightarrow|3,2\rangle$. In Fig. 14 we have plotted the transition rates for these four processes as a function of the magnetic field. The channel $|0,2\rangle$ represents two atoms in the identical atomic hyperfine state $|1,-1\rangle_{A}$. This is a high-field-seeking state; hence, transition 1 leads directly to trap loss. Channel $|1,2\rangle$ denotes two atoms in different atomic hyperfine states, with the low-field quantum numbers $\left|F_{1} M_{F_{1}} F_{2} M_{F_{2}}\right\rangle=|1,1,2,1\rangle$. The channel $|2,2\rangle$ has low-ield quantum numbers $\left|F_{1} M_{F_{1}} F_{2} M_{F_{2}}\right\rangle=|1,0,2,2\rangle$. Both channels $|1,2\rangle$ and $|2,2\rangle$ consist of one low-field-seeking atom and one high-field-seeking atom [cf. Fig. (3)]. Hence, one atom is lost per collision process whenever $|1,2\rangle$ or $|2,2\rangle$ are the final channels. Assuming a magnetic field of $100 \mathrm{G}$, the number of atoms lost per unit time can then be calculated from

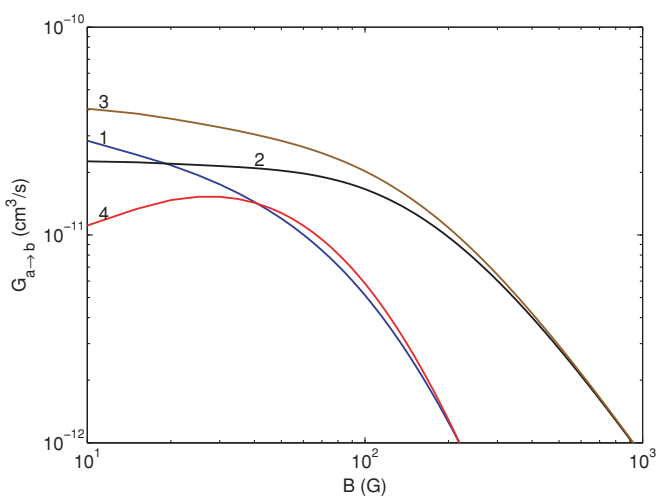

FIG. 14. (Color online) Transition rates $G_{a \rightarrow b}(B)$ for the following transitions: $1,|4,2\rangle \rightarrow|0,2\rangle ; 2,|4,2\rangle \rightarrow|1,2\rangle ; 3,|4,2\rangle \rightarrow$ $|2,2\rangle$; and $4,|4,2\rangle \rightarrow|3,2\rangle$. Values at $B=100 \mathrm{G}$ are used to calculate the trap loss and the time scale for population of different hyperfine states in Eqs. (21) and (22). The initial channel $|4,2\rangle$ represents two atoms with large positive Zeeman shifts and low-field quantum numbers $F_{1}=F_{2}=2$ together with $M_{F}=M_{F_{1}}+M_{F_{2}}=2$.
Eq. (16):

$$
\begin{aligned}
\frac{d \rho}{d t} & =-\rho^{2} \sum_{i=0}^{3} g_{i} G_{4 \rightarrow i} \simeq-5 \times 10^{-11} \rho^{2} \mathrm{~cm}^{3} \mathrm{~s}^{-1} \\
& =-5.0 \times 10^{13} \mathrm{~cm}^{-3} / \mathrm{s}^{-1} .
\end{aligned}
$$

The sum of the rates $\sum_{i} G_{4 \rightarrow i}$ is approximately $5 \times$ $10^{-11} \mathrm{~cm}^{3} / \mathrm{s}$ with dominant contributions from transitions 2 and 3 (cf. Fig. 14). The meaning of $g_{i}$ is to count the number of atoms lost in transition $|4,2\rangle \rightarrow|i, 2\rangle$; hence, $g_{0}=2$, $g_{1}=g_{2}=1$, and $g_{3}=0$. However, the atoms that remain trapped will evolve into a mixture of the three relevant and trappable atomic hyperfine states, that is, $|2,1\rangle,|2,2\rangle$, and $|2,0\rangle$, on a time scale of the order $T_{\text {ex }}$. We can estimate $T_{\text {ex }}$ from the transition rate for process 4 , since the other transitions $(1,2,3)$ primarily induce trap loss. In terms of the low-field quantum numbers, process 4 can be written (cf. Table I) $|2,1,2,1\rangle \rightarrow|2,0,2,2\rangle$. Following Eq. (18), we find

$$
T_{\mathrm{ex}}=\frac{1}{\rho G_{4 \rightarrow 3}} \simeq \frac{1}{\rho 6 \times 10^{-12} \mathrm{~cm}^{3}} \simeq 0.17 \mathrm{~s} .
$$

A rough estimate shows that $T_{\text {loss }}$ is of the same order. The fact that $T_{\text {loss }}$ and $T_{\text {ex }}$ are of the same order in this example is not surprising as the relevant rates are similar. This is seen from Fig. 14. From the same figure we also conclude that at higher magnetic fields, the rate for transition 4 is several orders of magnitude smaller than the rates for transitions 2 and 3 ; that is, $T_{\mathrm{ex}}>T_{\text {loss }}$. Hence, a strong magnetic field and the corresponding Zeeman interaction contributes as a stabilizing mechanism for the trap. Both time scales $T_{\mathrm{ex}}$ and $T_{\text {loss }}$ are important for experiments. A carefully prepared initial population of atoms will decay on the time scale $T_{\text {loss }}$ and be destroyed by population of unwanted atomic hyperfine states on the time scale $T_{\mathrm{ex}}$. In the present example, both effects are present and destroy the initial population rapidly. As seen in Fig. 14, the trap loss and the mixing time can be increased by more than one order of magnitude by working at higher magnetic field strengths.

\section{CONCLUSIONS}

In summary, we have calculated the $s$-wave scattering length for homonuclear collisions between atoms in identical and different atomic hyperfine states as a function of the magnetic field strength. Channel states with the proper symmetry under exchange of identical nuclei were used. We have searched for Feshbach resonances and accurately determined the magnetic field positions for 20 Feshbach resonances in ${ }^{39} \mathrm{~K}$ and 13 in ${ }^{41} \mathrm{~K}$ (see Tables II and III). Resonance widths and additional properties were obtained and are included in the tables. A brief study of the weakly bound states in the ${ }^{39} \mathrm{~K}_{2}$ molecule was also performed. It was found that the majority of the calculated Feshbach resonances can be explained within a model involving only two vibrational levels, with hyperfine structure and Zeeman interaction. Furthermore, we find large differences in the widths and the positions of the Feshbach resonances for the two isotopes. The ${ }^{41} \mathrm{~K}$ system has a small hyperfine splitting relative to the ${ }^{39} \mathrm{~K}$ system and a weaker hyperfine interaction. As a consequence, the resonance widths for ${ }^{41} \mathrm{~K}$ are significantly smaller than those for ${ }^{39} \mathrm{~K}$. 
Transition rate constants as a function of the magnetic field were obtained for both potassium isotopes. Results for selected channels have been presented. Generally, the transition rates as a function of the magnetic field strength show a rather complex and unpredictable behavior. The transitions rates for ${ }^{39} \mathrm{~K}$ are of the order of $10^{-11}-10^{-13} \mathrm{~cm}^{3} / \mathrm{s}$ at small magnetic field strengths, whereas the corresponding rates for ${ }^{41} \mathrm{~K}$ an order of magnitude smaller. We have identified the most probable transitions $\left|n, M_{F}\right\rangle \rightarrow\left|m, M_{F}\right\rangle$ (alternatively written as $\left|F_{1} M_{F_{1}} F_{2} M_{F_{2}}\right\rangle \rightarrow\left|F_{1}^{\prime} M_{F_{1}}^{\prime} F_{2}^{\prime} M_{F_{2}}^{\prime}\right\rangle$ at low magnetic field strengths) and also the most energetic transitions. We have shown how these rates affect the lifetime of a trapped atomic gas. Finally, it is our hope that the present results may be useful for future investigations of ultracold atomic and molecular systems.

\section{ACKNOWLEDGMENTS}

The NOTUR computing facilities have been used for the rather extensive numerical calculations in the present work.
[1] S. C. Doret, C. B. Connolly, W. Ketterle, and J. M. Doyle, Phys. Rev. Lett. 103, 103005 (2009).

[2] A. Fioretti, D. Comparat, A. Crubellier, O. Dulieu, F. MasnouSeeuws, and P. Pillet, Phys. Rev. Lett. 80, 4402 (1998).

[3] A. N. Nikolov, E. E. Eyler, X. T. Wang, J. Li, H. Wang, W. C. Stwalley, and P. L. Gould, Phys. Rev. Lett. 82, 703 (1999).

[4] T. Takekoshi, B. M. Patterson, and R. J. Knize, Phys. Rev. Lett. 81, 5105 (1998).

[5] R. Wynar, R. S. Freeland, D. J. Han, C. Ryu, and D. J. Heinzen, Science 287, 1016 (2000).

[6] H. Fesbhach, Ann. Phys. (NY) 5, 357 (1958).

[7] S. Jochim, M. Bartenstein, A. Altmeyer, G. Hendl, S. Riedl, C. Chin, J. H. Denschlag, and R. Grimm, Science 302, 2101 (2003).

[8] J. R. Anglin and W. Ketterle, Nature (London) 416, 211 (2002).

[9] T. Kraemer et al., Nature (London) 440, 315 (2006).

[10] K. Huang and C. N. Yang, Phys. Rev. 105, 767 (1957).

[11] C. D'Errico, M. Zaccanti, M. Fattori, G. Roati, M. Inguscio, G. Modugno, and A. Simoni, New J. Phys. 9, 223 (2007).

[12] L. DeSarlo, P. Maioli, G. Barontini, J. Catani, F. Minardi, and M. Inguscio, Phys. Rev. A 75, 022715 (2007).

[13] J. L. Bohn, J. P. Burke, C. H. Greene, H. Wang, P. L. Gould, and W. C. Stwalley, Phys. Rev. A 59, 3660 (1999).

[14] M. Gacesa, P. Pellegrini, and R. Cote, Phys. Rev. A 78, 010701(R) (2008).

[15] M. Lysebo and L. Veseth, Phys. Rev. A 79, 062704 (2009).

[16] J. Aldegunde, B. A. Rivington, P. S. Zuchowski, and J. M. Hutson, Phys. Rev. A 78, 033434 (2008).
[17] M. Abramowitz and I. A. Stegun, Handbook of Mathematical Functions with Formulas, Graphs, and Mathematical Tables (Dover, New York, 1964).

[18] B. Gao, Phys. Rev. A 54, 2022 (1996).

[19] H. T. C. Stoof, J. M. V. A. Koelman, and B. J. Verhaar, Phys. Rev. B 38, 4688 (1988).

[20] S. Falke, H. Knockel, J. Friebe, M. Riedmann, E. Tiemann, and C. Lisdat, Phys. Rev. A 78, 012503 (2008).

[21] L. D. Landau and E. M. Lifshitz, Quantum Mechanics: Nonrelativistic theory (Pergamon Press, Oxford, New York, 1989), 3rd ed., Vol. 3.

[22] E. Wigner and E. E. Witmer, Zeits. Phys. 51, 859 (1928).

[23] D. E. Manolopoulos, J. Chem. Phys. 85, 6425 (1986).

[24] B. R. Johnson, J. Comput. Phys. 13, 445 (1973).

[25] E. Arimondo, M. Inguscio, and P. Violino, Rev. Mod. Phys. 49, 31 (1977).

[26] G. Audi, A. H. Wapstra, C. Thibault, J. Blachot, and O. Bersillon, Nucl. Phys. A729, 337 (2003).

[27] E. Tiesinga, B. J. Verhaar, and H. T. C. Stoof, Phys. Rev. A 47, 4114 (1993).

[28] R. M. C. Ahn, J. P. H. W. v.d.Eijnde, and B. J. Verhaar, Phys. Rev. B 27, 5424 (1983).

[29] A. Lagendijk, I. F. Silvera, and B. J. Verhaar, Phys. Rev. B 33, 626 (1986).

[30] E. Tiesinga, S. J. M. Kuppens, B. J. Verhaar, and H. T. C. Stoof, Phys. Rev. A 43, 5188 (1991).

[31] M. Houbiers, H. T. C. Stoof, W. I. McAlexander, and R. G. Hulet, Phys. Rev. A 57, R1497 (1998). 


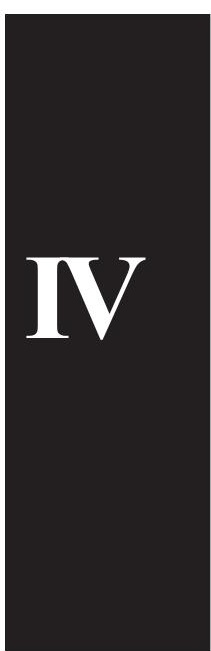


This article is removed. 
